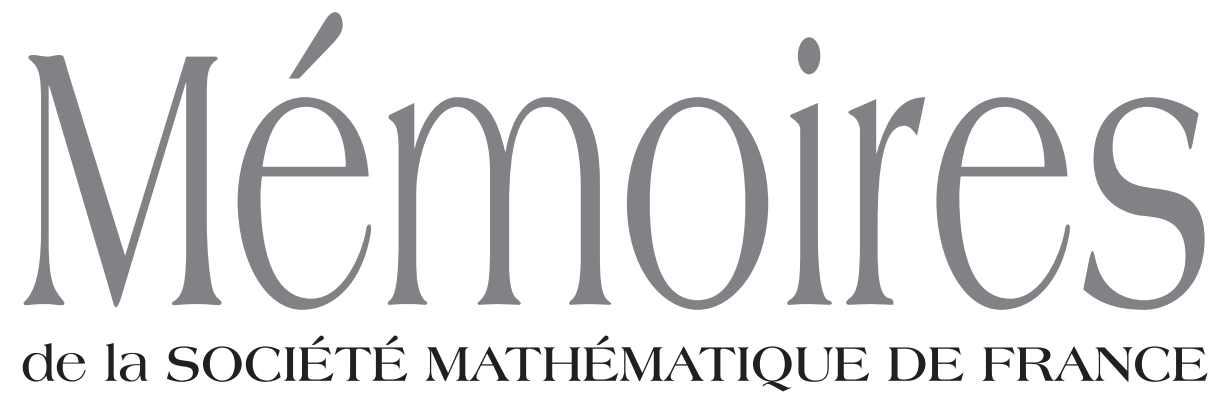

Numéro 120 Nouvelle série
CONVERGENCE DES POLYGONES DE HARDER-NARASIMHAN

\title{
H. Chen
}

20010 


\title{
Comité de rédaction
}

\author{
Jean BARGE \\ Emmanuel BREUILLARD \\ Gérard BESSON \\ Antoine CHAMBERT-LOIR \\ Jean-François DAT \\ Raphaël KRIKORIAN (dir.) \\ Charles FAVRE \\ Daniel HUYBRECHTS \\ Yves LE JAN \\ Laure SAINT-RAYMOND \\ Wilhem SCHLAG
}

\section{Diffusion}

Maison de la SMF

Case 916 - Luminy

13288 Marseille Cedex 9

France

smf@smf .univ-mrs.fr
Hindustan Book Agency

O-131, The Shopping Mall

Arjun Marg, DLF Phase 1

Gurgaon 122002, Haryana

Inde
AMS

P.O. Box 6248

Providence RI 02940

USA

WwW . ams . org

\section{Tarifs}

Vente au numéro : $28 €(\$ 42)$

Abonnement Europe : $255 €$, hors Europe : $290 €(\$ 435)$

Des conditions spéciales sont accordées aux membres de la SMF.

\section{Secrétariat : Nathalie Christiaën}

Mémoires de la SMF

Société Mathématique de France

Institut Henri Poincaré, 11, rue Pierre et Marie Curie

75231 Paris Cedex 05, France

Tél : (33) 0144276799 • Fax : (33) 0140469096

revues@smf.ens.fr • http://smf.emath.fr/

(C) Société Mathématique de France 2010

Tous droits réservés (article L 122-4 du Code de la propriété intellectuelle). Toute représentation ou reproduction intégrale ou partielle faite sans le consentement de l'éditeur est illicite. Cette représentation ou reproduction par quelque procédé que ce soit constituerait une contrefaçon sanctionnée par les articles L 335-2 et suivants du CPI.

ISSN 0249-633-X

ISBN 978-2-85629-296-9

Directrice de la publication : Aline BONAMI 


\section{CONVERGENCE DES POLYGONES DE HARDER-NARASIMHAN}

Huayi Chen 
H. Chen

Université Paris Diderot — Paris 7, Institut de mathématiques de Jussieu.

E-mail : chenhuayi@math.jussieu.fr

Classification mathématique par sujets (2000). - 14G40, 14F05.

Mots-clefs. - Géométrie d'Arakelov, méthode de pentes, filtration, polygone de Harder-Narasimhan, théorème de Hilbert-Samuel. 


\title{
CONVERGENCE DES POLYGONES DE HARDER-NARASIMHAN
}

\author{
Huayi Chen
}

Résumé. - On interprète la théorie des polygones de Harder-Narasimhan par le langage des $\mathbb{R}$-filtrations. En utilisant une variante du lemme de Fekete et un argument combinatoire des monômes, on établit la convergence uniforme des polygones associés à une algèbre graduée munie de filtrations. Cela conduit à l'existence de plusieurs invariants arithmétiques dont un cas très particulier est la capacité sectionnelle. Deux applications de ce résultat en géométrie d'Arakelov sont abordées : le théorème de Hilbert-Samuel arithmétique ainsi que l'existence et l'interprétation géométrique de la pente maximale asymptotique.

Abstract (Convergence of Harder-Narasimhan polygons). - We interpret the theory of Harder-Narasimhan polygons by the language of $\mathbb{R}$-filtrations. By using a variant version of Fekete's lemma and a combinatoric argument on monomials, we establish the uniform convergence of polygons associated to a graded algebra equipped with filtrations. This leads to the existence of several arithmetic invariants a very particular case of which is the sectional capacity. Two applications in Arakelov geometry are developed: the arithmetic Hilbert-Samuel theorem and the existence and the geometric interpretation of the asymptotic maximal slope. 



\section{TABLE DES MATIĖRES}

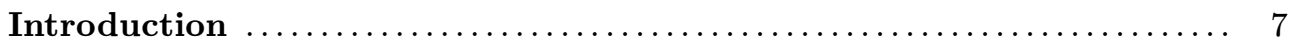

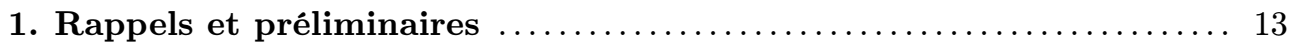

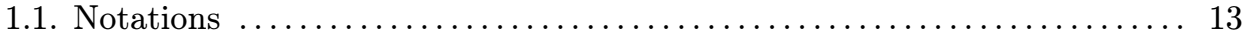

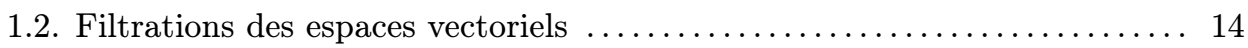

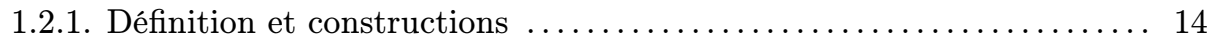

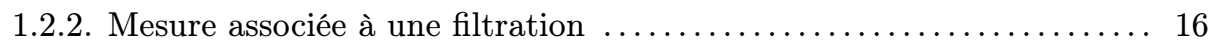

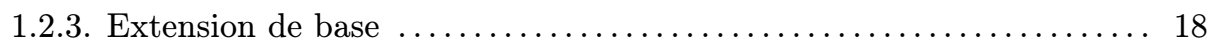

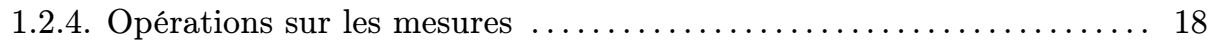

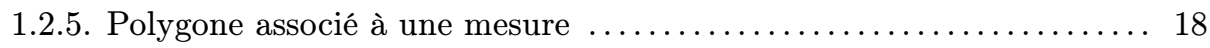

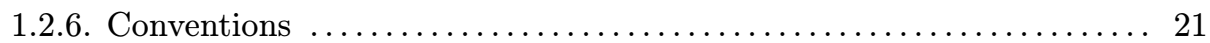

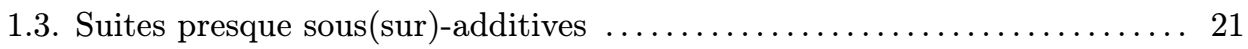

1.4. Quelques faits dans la géométrie algébrique $\ldots \ldots \ldots \ldots \ldots \ldots \ldots \ldots \ldots$

2. Filtrations de Harder-Narasimhan $\ldots \ldots \ldots \ldots \ldots \ldots \ldots \ldots \ldots \ldots \ldots$

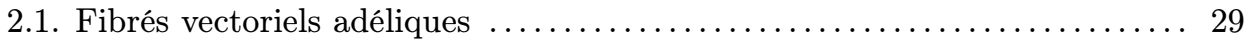

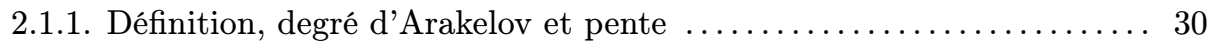

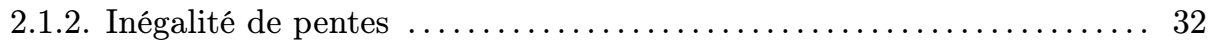

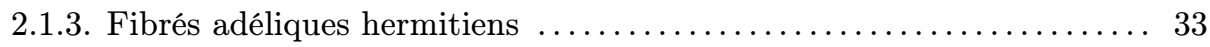

2.1.4. Pente minimale du produit tensoriel $\ldots \ldots \ldots \ldots \ldots \ldots \ldots \ldots \ldots \ldots \ldots$

2.1.5. Comparaison des fibrés vectoriels adéliques aux fibrés adéliques

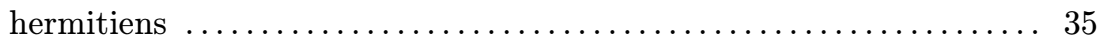

2.1.6. Application à l'étude du produit tensoriel $\ldots \ldots \ldots \ldots \ldots \ldots \ldots \ldots \ldots$

2.1.7. Produit tensoriel avec un fibré inversible adélique ............ 38

2.2. Filtration et polygone de Harder-Narasimhan $\ldots \ldots \ldots \ldots \ldots \ldots \ldots \ldots . \ldots \ldots$

2.2.1. Semi-stabilité et drapeau de Harder-Narasimhan . . . . . . . . . . 38

2.2.2. Filtration de Harder-Narasimhan . . . . . . . . . . . . . . . . . . 39

2.2.3. Polygone de Harder-Narasimhan $\ldots \ldots \ldots \ldots \ldots \ldots \ldots \ldots \ldots \ldots \ldots \ldots \ldots$

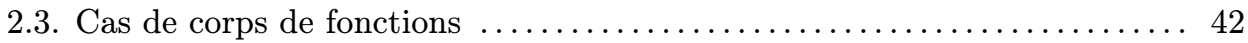

2.3.1. Fibré vectoriel adélique sur un corps de fonctions . ........... 42

2.3.2. Pentes, filtration et polygone de Harder-Narasimhan . . . . . . . . . 44

3. Convergence des polygones $\ldots \ldots \ldots \ldots \ldots \ldots \ldots \ldots \ldots \ldots \ldots \ldots \ldots$

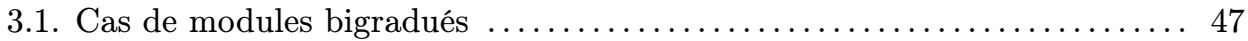


3.1.1. Séries de Poincaré à deux variables $\ldots \ldots \ldots \ldots \ldots \ldots \ldots \ldots \ldots \ldots$

3.1.2. Mesures associées à une série à deux variables $\ldots \ldots \ldots \ldots \ldots \ldots \ldots 51$

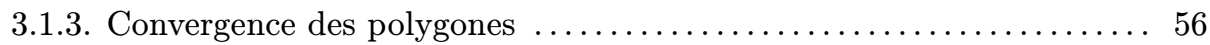

3.2. Algèbres graduées quasi-filtrées et pseudo-filtrées $\ldots \ldots \ldots \ldots \ldots \ldots \ldots \ldots 7$

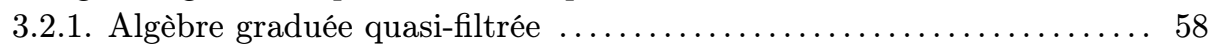

3.2.2. Algèbre graduée pseudo-filtrée $\ldots \ldots \ldots \ldots \ldots \ldots \ldots \ldots \ldots \ldots \ldots \ldots \ldots \ldots \ldots \ldots \ldots \ldots$

3.3. Convergence des mesures : cas d'une algèbre de polynômes $\ldots \ldots \ldots \ldots \ldots 61$

3.3.1. Combinatoire des points entiers dans des simplexes $\ldots \ldots \ldots \ldots \ldots .62$

3.3.2. Convergence vague des mesures $\ldots \ldots \ldots \ldots \ldots \ldots \ldots \ldots \ldots \ldots 6 . \ldots \ldots 6$

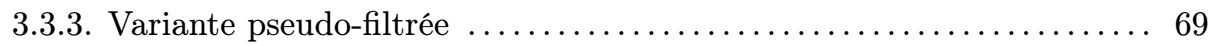

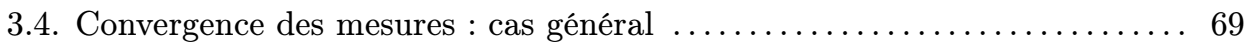

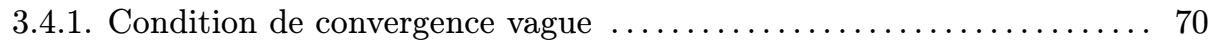

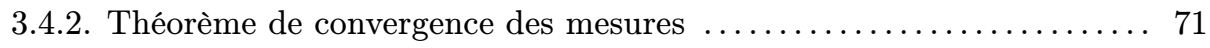

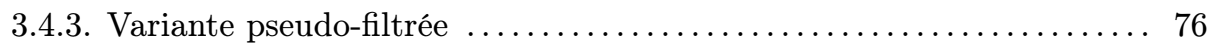

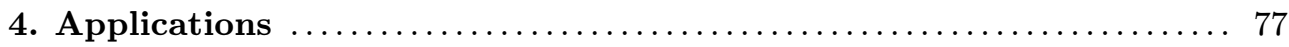

4.1. Théorème de Hilbert-Samuel arithmétique $\ldots \ldots \ldots \ldots \ldots \ldots \ldots \ldots \ldots 7$

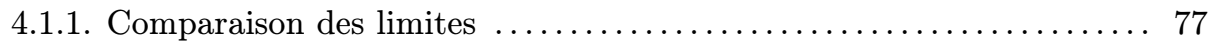

4.1.2. Algèbre en fibrés vectoriels adéliques $\ldots \ldots \ldots \ldots \ldots \ldots \ldots \ldots \ldots \ldots$

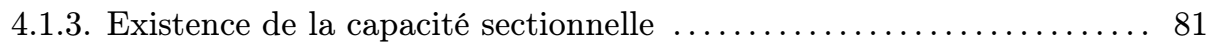

4.1.4. Théorème de Hilbert-Samuel arithmétique $\ldots \ldots \ldots \ldots \ldots \ldots \ldots \ldots . \ldots 3$

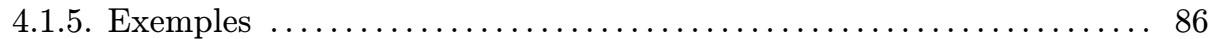

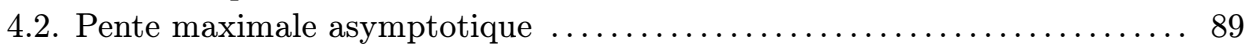

4.2.1. Sur-additivité de la pente maximale asymptotique $\ldots \ldots \ldots \ldots \ldots . . \ldots 0$

4.2.2. Pente maximale asymptotique d'un fibré inversible hermitien

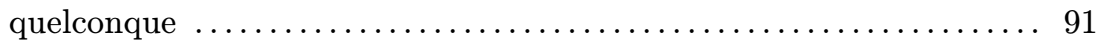

4.2.3. Pente maximale asymptotique d'un fibré vectoriel hermitien ...... 96

4.2.4. Lien avec une condition d'annulation $\ldots \ldots \ldots \ldots \ldots \ldots \ldots \ldots \ldots \ldots$

4.3. Polygones et pentes asymptotiques en géométrie relative . . . . . . . . 103

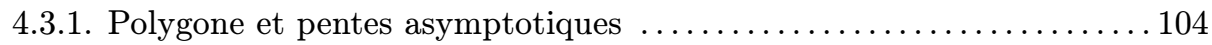

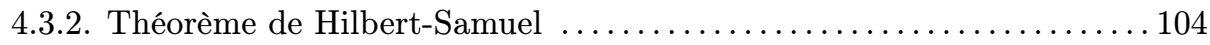

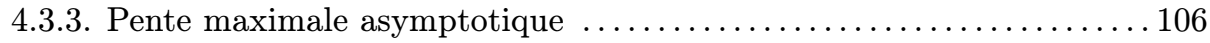

4.3.4. Pente maximale asymptotique et annulation de section globale . . . 110

4.3.5. Pente maximale asymptotique d'un fibré vectoriel $\ldots \ldots \ldots \ldots \ldots \ldots 111$

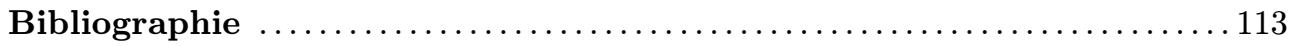




\section{INTRODUCTION}

Dans l'approximation diophantienne, on s'intéresse à étudier les solutions rationnelles d'un système d'équations polynomiales à coefficients dans un corps de nombres. L'approche d'Arakelov, qui est une combinaison de la théorie des schémas à la Grothendieck avec la géométrie complexe hermitienne, fournit un cadre géométrique bien adapté aux équations polynomiales en tenant compte les métriques.

Un formalisme important dans ce cadre est l'application d'évaluation et la méthode de pentes dues à Bost $[\mathbf{6}, \mathbf{9}]$. Étant donnés un corps de nombres $K$, une variété projective $X$ définie sur $K$ et un faisceau inversible ample $L$ sur $X$, l'espace $H^{0}(X, L)$ peut être considéré comme un espace de "polynômes homogènes". L'application d'évaluation (notée $\mathrm{EV}_{\Sigma, L}$ ) est un homomorphisme de $H^{0}(X, L)$ vers $H^{0}\left(\Sigma,\left.L\right|_{\Sigma}\right)$ défini par restriction des sections à $\Sigma$, où $\Sigma$ est un sous- $K$-schéma fermé de $X$. Cela généralise la construction classique qui consiste à évaluer les valeurs des polynômes homogènes en un ou plusieurs points rationnels.

Quitte à choisir un modèle entier de $(X, L)$ et une métrique hermitienne sur $L_{\mathbb{C}}$, la source et le but de l'application $\mathrm{EV}_{\Sigma, L}$ deviennent des espaces vectoriels sur $K$ associés à certains fibrés vectoriels hermitiens sur Spec $\theta_{K}$, où $\theta_{K}$ est l'anneau des entiers dans $K$. On obtient, en utilisant les inégalités de pentes, des éléments nonnuls dans $H^{0}(X, L)$ dont l'image par $\mathrm{EV}_{\Sigma, L}$ est nulle. Classiquement ces éléments sont appelés des "polynômes auxiliaires" qui sont des objets essentiels dans l'approximation diophantienne, souvent construits par le lemme de Siegel.

L'intérêt de la méthode de pentes est de transformer une "démonstration de transcendence" à une seule inégalité. Cette inégalité, dont les ingrédients sont des invariants arithmétiques naturellement définis, comme la hauteur d'un homomorphisme $K$-linéaire, le degré d'Arakelov et la pente d'un fibré vectoriel hermitien, permet de séparer les contributions de différents termes, et de donner un cadre plus souple pour diverses méthodes d'estimation.

Dans de nombreuses applications, on considère une suite d'applications d'évaluation $\mathrm{EV}_{\Sigma, L} \otimes n$ et étudie leur comportement asymptotique lorsque $n$ tend vers l'infini. Il est donc nécessaire de comprendre le comportement asymptotique des fibrés vectoriels hermitiens dont les espaces vectoriels sous-jacents sont les $H^{0}\left(X, L^{\otimes n}\right)$.

Le premier résultat dans cette direction est le théorème de Hilbert-Samuel arithmétique dû à Gillet et Soulé [23]. Soient $(\mathscr{X}, \mathscr{L})$ un modèle entier de $(X, L)$ et $\pi: \mathscr{X} \rightarrow \operatorname{Spec} \bigoplus_{K}$ le morphisme structurel. Si on munit l'espace vectoriel $H^{0}\left(X, L^{\otimes n}\right)$ 
des sup-normes, le $\theta_{K^{-}}$module $\pi_{*}\left(\mathscr{L}^{\otimes n}\right)$ peut être considéré comme un réseau dans un espace vectoriel normé. Gillet et Soulé ont montré que, si les métriques sur $L$ sont positives, alors

$$
\lim _{n \rightarrow+\infty} \frac{(d+1) !}{n^{d+1}} \chi\left(\pi_{*}\left(\mathscr{L}^{\otimes n}\right),\|\cdot\|_{\text {sup }}\right)=\widehat{c}_{1}(\overline{\mathscr{L}})^{d+1}
$$

où $d=\operatorname{dim} X, \widehat{c}_{1}(\overline{\mathscr{L}})^{d+1}$ est le nombre d'intersection arithmétique et $\chi$ est la caractéristique d'Euler-Poincaré, qui est le logarithme du volume de la boule unité divisé par le covolume du réseau. Une reformulation de ce résultat est

$$
\lim _{n \rightarrow+\infty} \widehat{\mu}\left(\overline{\pi_{*}\left(\mathscr{L}^{\otimes n}\right)}\right) / n=\frac{\widehat{c}_{1}\left(\overline{\mathscr{L}}^{d+1}\right)}{[K: \mathbb{Q}](d+1) c_{1}(L)^{d}},
$$

où $\widehat{\mu}$ est la fonction de pente, qui est le degré d'Arakelov normalisé divisé par le rang.

Bien que les autres pentes comme par exemple la pente maximale $\widehat{\mu}_{\text {max }}$, qui est la valeur maximale des pentes des sous-fibrés, et la pente minimale $\widehat{\mu}_{\text {min }}$, qui est la valeur minimale des pentes des fibrés quotients, interviennent aussi naturellement dans les inégalités de pentes, on sait relativement peu sur leur comportement asymptotique. Par exemple, dans [7], Bost a démontré que les pentes maximales $\widehat{\mu}_{\max }\left(S^{n} \bar{E}\right)$ croissent linéairement lorsque $n$ tend vers l'infini. Il a aussi obtenu des majorations de ces pentes maximales. Mais on ne sait pas en général si la limite des $\widehat{\mu}_{\max }\left(S^{n} \bar{E}\right) / n$ existe dans $\mathbb{R}$. L'une des difficultés est que, contrairement à la fonction de pente $\widehat{\mu}$, en général la pente maximale et la pente minimale n'admettent pas d'additivité par rapport aux suites exactes courtes, qui est une condition importante dans la technique de dévissage.

Le but de cet article est alors d'étudier le comportement asymptotique des fibrés vectoriels hermitiens. En particulier, on établit la convergence des suites $\left(\widehat{\mu}_{\max }\left(\overline{\pi_{*}\left(\mathscr{L}^{\otimes n}\right)}\right) / n\right)_{n \geqslant 1}$ et $\left(\widehat{\mu}_{\min }\left(\overline{\pi_{*}\left(\mathscr{L}^{\otimes n}\right)}\right) / n\right)_{n \geqslant 1}$. Pour surmonter la difficulté d'absence de l'additivité, on utilise la technique de polygone de Harder-Narasimhan avec le point de vue des $\mathbb{R}$-filtrations et des mesures. Le polygone de HarderNarasimhan est initialement une notion en géométrie algébrique, proposée par Harder et Narasimhan [28]. En géométrie d'Arakelov, cette notion est introduite par Stuhler [42] et Grayson [24]. Si $\bar{E}$ est un fibré vectoriel hermitien sur Spec $\theta_{K}$, le polygone de Harder-Narasimhan de $\bar{E}$ est par définition la fonction concave sur $[0, \mathrm{rg} E]$ dont le graphe est le bord supérieur de l'enveloppe convexe des points $\left(\operatorname{rg} F, \widehat{\operatorname{deg}}_{n}(\bar{F})\right)$, où $\widehat{\operatorname{deg}}_{n}(\bar{F})$ est le degré d'Arakelov normalisé de $F$. On désigne par $\mathscr{P}_{\bar{E}}$ la forme normalisée de ce polygone, c'est-à-dire la fonction définie sur $[0,1]$ dont le graphe est similaire à celui du polygone de Harder-Narasimhan. L'avantage du polygone normalisé est qu'il permet d'étudier les diverses pentes en même temps :

$$
\widehat{\mu}(\bar{E})=\mathscr{P}_{\bar{E}}(1), \quad \widehat{\mu}_{\max }(\bar{E})=\lim _{t \rightarrow 0+} \mathscr{P}_{\bar{E}}^{\prime}(t), \quad \widehat{\mu}_{\min }(\bar{E})=\lim _{t \rightarrow 1-} \mathscr{P}_{\bar{E}}^{\prime}(t) .
$$

Les sommets du polygone de Harder-Narasimhan correspondent à un drapeau de $E$ :

$$
E=E_{0} \supsetneq E_{1} \supsetneq \cdots \supsetneq E_{d}=0
$$


tel que les sous-quotients $\overline{E_{i} / E_{i+1}}$ soient semi-stables (c'est-à-dire $\mu_{i}:=\widehat{\mu}\left(\overline{E_{i} / E_{i+1}}\right)$ coïncide avec $\left.\widehat{\mu}_{\max }\left(\overline{E_{i} / E_{i+1}}\right)\right)$ et que les pentes successives $\mu_{i}$ satisfassent aux inégalités $\mu_{0}<\cdots<\mu_{d-1}$. Classiquement le drapeau comme ci-dessus s'appelle la filtration de Harder-Narasimhan de $\bar{E}$. Mais la donnée d'un drapeau et d'une suite strictement croissante de nombres réels de la même longueur définit en fait une $\mathbb{R}$-filtration décroissante. Dans la figure 1 , le graphe à gauche représente la dérivée

Figure 1. Dérivé du polygone normalisé et la fonction de distribution
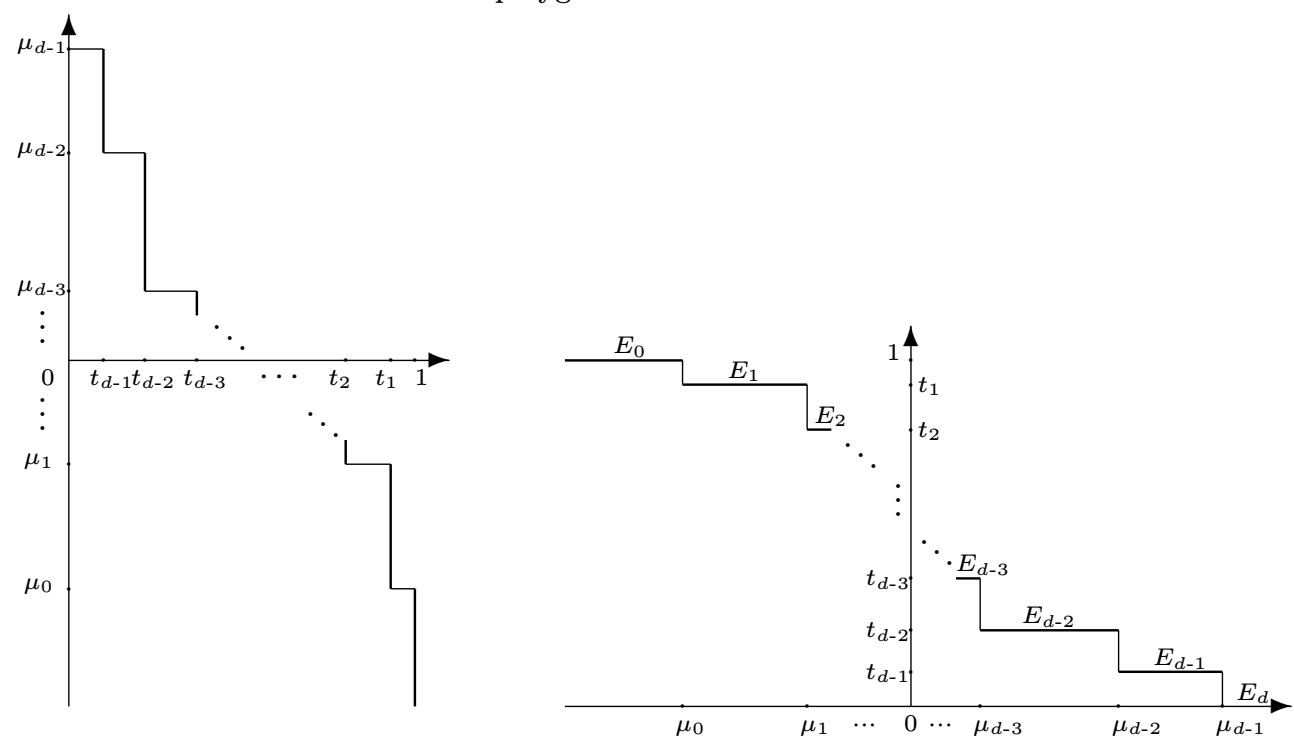

du premier ordre de la fonction $\mathscr{P}_{\bar{E}}$, où $t_{i}=\operatorname{rg} E_{i} / \operatorname{rg} E$. C'est une fonction d'escalier définie sur $[0,1]$. Le graphe à droite représente la fonction inverse de la fonction dans le graphe à gauche. C'est une fonction décroissante d'escalier définie sur $\mathbb{R}$ qui prend valeurs dans $[0,1]$. Elle définit donc une mesure de probabilité borélienne $\nu_{\bar{E}}$ sur $\mathbb{R}$. Si on place convenablement les $E_{i}$, comme présenté dans le graphe à droite, on obtient une $\mathbb{R}$-filtration décroissante de $E$ qui induit par changement de scalaire à $K$ une $\mathbb{R}$-filtration décroissante $\mathcal{F}^{\bar{E}}$ de $E_{K}$.

Réciproquement, si on se donne un espace vectoriel de rang fini et non-nul $V$ sur $K$ muni d'une $\mathbb{R}$-filtration $\mathcal{F}$, alors la fonction $x \mapsto \operatorname{rg}\left(\mathcal{F}_{x} V\right) / \operatorname{rg} V$ est une fonction d'escalier décroissante, comme celle dans le graphe à droite. L'intégrale de son inverse définit une fonction concave et linéaire par morceaux — c'est-à-dire un polygone sur $[0,1]$. En résumé, on a des applications naturelles

$$
\left\{\begin{array}{l}
\text { espaces vectoriels de } \\
\text { rang fini } \mathbb{R} \text {-filtrés }
\end{array}\right\} \rightarrow\left\{\begin{array}{l}
\text { mesures de probabilité boréliennes } \\
\text { sur } \mathbb{R} \text { combinaisons linéaires de } \\
\text { mesures de Dirac }
\end{array}\right\} \leftrightarrow\left\{\begin{array}{l}
\text { polygones } \\
\text { sur }[0,1]
\end{array}\right\},
$$


dont la dernière est une bijection, qui envoie $\nu_{\bar{E}}$ en $\mathscr{P}_{\bar{E}}$. Cela nous permet d'utiliser les espaces $\mathbb{R}$-filtrés et les mesures boréliennes sur $\mathbb{R}$ à étudier les fibrés vectoriels hermitiens. En outre, des constructions similaires existent dans le cadre géométrique des fibrés vectoriels sur une courbe.

L'algèbre $B=\bigoplus_{n \geqslant 0} H^{0}\left(X, L^{\otimes n}\right)$ des sections globales, munie des filtrations de Harder-Narasimhan, est une algèbre graduée de type fini munie des $\mathbb{R}$-filtrations. On a expliqué plus haut que l'information des pentes et des polygones de HarderNarasimhan est complètement encodée dans les $\mathbb{R}$-filtrations de $B$. On propose deux stratégies différentes à étudier le comportement asymptotique de ces filtrations. La première est inspirée par un travail de Faltings et Wüstholz [20], qui est une généralisation de la théorie des séries de Poincaré - un instrument important à étudier les algèbres graduées, classiquement utilisée pour déterminer le comportement asymptotique des rangs des composantes homogènes. Bien que cette approche permet de calculer explicitement la limite des polygones, elle n'est valable que pour le cas particulier où les filtrations sont induites par une autre graduation de $B$. L'une des obstructions d'utiliser la méthode de série de Poincaré au cas général est que, dans une algèbre graduée, le degré du produit de deux éléments homogènes est égal à la somme de leurs degrés; par contre, dans l'algèbre munie des filtrations qui nous intéresse, en général on sait seulement une minoration de la position d'un produit dans la filtration par la somme des positions de chaque élément, avec même un terme d'erreur. Cette observation conduit à la deuxième stratégie : suites presque sur-additives et lemme de Fekete. Le lemme de Fekete est une méthode classique à déterminer la convergence d'une suite sous(sur)-additive : si $\left(a_{n}\right)_{n \geqslant 1}$ est une suite réelle qui est sur-additive, à savoir $a_{n+m} \geqslant a_{n}+a_{m}$ quels que soient $m, n$, alors la suite $\left(a_{n} / n\right)_{n \geqslant 1}$ converge dans $\mathbb{R} \cup\{+\infty\}$, et la limite est finie lorsque $a_{n}=O(n)$. On établit d'abord la presque sur-additivité de la suite des mesures associées aux filtrations de l'algèbre graduée, et on en déduit la convergence vague des mesures dilatées en faisant appel à une variante du lemme de Fekete. Enfin on obtient la convergence uniforme des polygones par le lien entre les mesures et les polygones. Par une méthode similaire, l'existence des limites de pentes maximales et de pentes minimales est aussi obtenue.

Les résultats obtenus dans cet article peuvent être comparés à un travail de Rumely, Lau et Varley [41] sur l'existence des capacités sectionnelles. La capacité sectionnelle est un invariant qui généralise simultanément la capacité logarithmique d'un diviseur en géométrie complexe, et le nombre d'auto-intersection d'un fibré inversible hermitien en géométrie d'Arakelov. Le résultat sur la convergence des polygones que l'on obtient implique en particulier l'existence et la positivité de la capacité sectionnelle au cas des normes (dans [41], les auteurs considèrent un cadre plus général des semi-normes) et donc implique le théorème de Hilbert-Samuel arithmétique. De plus, la convergence des polygones est valable pour toute algèbre graduée de type fini munie de filtrations convenables. Donc le polygone limite est un invariant arithmétique qui existe dans un cadre très général et qui généralise de nombreux invariants intéressants. 
Dans le cas où $\mathscr{L}$ est ample et les métriques sur $L$ sont positives, la valeur en 1 de la limite des polygones est liée au nombre d'intersection arithmétique de $\overline{\mathscr{L}}$. Il est donc très naturel d'étudier les interprétations géométriques des autres invariants limites. Dans cet article, on propose une telle interprétation pour la pente maximale asymptotique $\widehat{\mu}_{\max }^{\pi}(\overline{\mathscr{L}})$ - la limite des $\widehat{\mu}_{\max }\left(\pi_{*}\left(\overline{\mathscr{L}}^{\otimes n}\right)\right) / n$ - par une propriété d'annulation de section de petite norme. En outre, on démontre que cette pente maximale asymptotique est sur-additive par rapport à $\overline{\mathscr{L}}$. Cette observation nous permet d'étendre son domaine de définition à l'ensemble de tous les fibrés inversibles hermitiens $\overline{\mathscr{L}}$ sur $\mathscr{X}$, même si les espaces $\pi_{*}\left(\mathscr{L}^{\otimes n}\right)$ se réduisent éventuellement à zéro. Cela montre que cet invariant a une souplesse à appliquer dans diverses situations. Le lien explicite avec la propriété d'annulation suggère des applications en géométrie algébrique et en géométrie d'Arakelov dans la future.

L'article est organisé comme la suite. Dans le premier chapitre, on rappelle d'abord quelques notations utilisées dans l'article; ensuite, un préliminaire sur les $\mathbb{R}$-filtrations est introduit, y compris la mesure et le polygone associés à une filtration, qui sont des outils importants; la section suivante est consacrée à quelques généralisations du lemme de Fekete; enfin, on rappelle des faits dans la géométrie algébrique que l'on utilisera plus loin. Le deuxième chapitre est un rappel sur la théorie des fibrés vectoriels adéliques, tout particulièrement sur les polygones de Harder-Narasimhan, qui sont des objets à étudier dans cet article. C'est dans le troisième chapitre que le résultat principal de l'article - la convergence des polygones - est démontré : la première section est consacrée au cas des algèbres bigraduées, où on développe la méthode de séries de Poincaré à deux variables; dans la deuxième section, on propose deux notions, algèbre graduée quasi-filtrée et algèbre graduée pseudo-filtrée, qui sont des algèbres graduées munies de filtrations où la convergence des polygones est susceptible d'être vraie; on démontre dans la troisième section cette convergence pour le cas particulier où l'algèbre graduée est une algèbre de polynômes, en utilisant un argument combinatoire fin sur les monômes et en faisant appel au lemme de Fekete généralisé appliqué aux mesures associées aux filtrations; enfin, dans la quatrième section, on démontre le cas général par réduction au cas précédent d'algèbres de polynômes. Le dernier chapitre est consacré aux applications de la théorie générale établie dans le troisième chapitre : on obtient l'existence de la capacité sectionnelle et donc le théorème d'Hilbert-Samuel arithmétique; ensuite, l'interprétation géométrique de la pente maximale asymptotique est discutée; enfin, on aborde l'analogue de ces résultats dans le cadre de la géométrie algébrique sur une courbe.

Une grande partie de résultats dans cet article est issue de ma thèse doctorale dirigée par J.-B. Bost. Je tiens à lui exprimer mes remerciements pour son encadrement et ses encouragements. Je voudrais exprimer mes gratitudes à C. Mourougane pour l'intérêt qu'il a manifesté à ce travail et pour les discussions que nous avons eues, et aussi à A. Chambert-Loir, D. Rössler et C. Soulé pour leurs remarques sur une version précédente de cet article sous forme d'une partie de ma thèse. Je suis reconnaissant à É. Gaudron qui m'a invité à une rencontre de l'ANR 
《Autour de la théorie des pentes» organisée à l'Institut Fourier où j'ai appris de lui la théorie des fibrés vectoriels adéliques. Enfin, je remercie sincèrement le/la rapporteur/rapporteuse pour sa lecture soigneuse et également pour ses suggestions et corrections qui m'ont beaucoup aidé à améliorer le manuscrit. 


\section{CHAPITRE 1}

\section{RAPPELS ET PRÉLIMINAIRES}

\subsection{Notations}

Dans cet article, sauf mention au contraire, $K$ désigne un corps de nombres dont l'anneau des entiers est noté $\theta_{K}$. On désigne par $\Sigma_{f}$ l'ensemble des places finies de $K$, qui s'identifie à l'ensemble des points fermés dans $\operatorname{Spec} \theta_{K}$. Soit $\Sigma_{\infty}$ l'ensemble des places infinie de $K$, qui se décompose en une union disjointe de deux parties : $\Sigma_{\infty, r}$ des places réelles et $\Sigma_{\infty, c}$ des places complexes. Soit $\Sigma=\Sigma_{f} \cup \Sigma_{\infty}$.

Si $\mathfrak{p} \in \Sigma_{f}$ est une place finie de $K$, on désigne par $v_{\mathfrak{p}}: K^{\times} \rightarrow \mathbb{Z}$ la valuation discrète associée à $\mathfrak{p}$. Soient $\mathbb{F}_{\mathfrak{p}}=\Theta_{K} / \mathfrak{p}$ le corps résiduel de $\mathfrak{p}$ et $|\cdot|_{\mathfrak{p}}$ la valeur absolue sur $K$ telle que $|a|_{\mathfrak{p}}=p^{-v_{\mathfrak{p}}(a)}$ quel que soit $a \in K^{\times}$, où $p$ est la caractéristique de $\mathbb{F}_{\mathfrak{p}}$. On note $n_{\mathfrak{p}}=\left[\mathbb{F}_{\mathfrak{p}}: \mathbb{Z} / p \mathbb{Z}\right]$.

Chaque place infinie $v \in \Sigma_{\infty}$ correspond à un plongement $\sigma: K \rightarrow \mathbb{C}$, unique à conjugaison complexe près. On désigne par $|\cdot|_{v}$ la valeur absolue sur $K$ définie par $|a|_{v}=|\sigma(a)|$, où $|\cdot|$ est la valeur absolue usuelle sur $\mathbb{C}$. On définit $n_{v}=1$ si $v \in \Sigma_{\infty, r}$ et $n_{v}=2$ si $v \in \Sigma_{\infty, c}$.

La formule de produit comme ci-dessous est importante dans la théorie des nombres : si $a$ est un élément non-nul de $K$, alors, pour toute sauf un nombre fini de places $\mathfrak{p} \in \Sigma_{f},|a|_{\mathfrak{p}}=1$; de plus, on a

$$
\prod_{v \in \Sigma_{f} \cup \Sigma_{\infty}}|a|_{v}^{n_{v}}=1 .
$$

Pour toute place $v \in \Sigma_{f} \cup \Sigma_{\infty}$, on désigne par $K_{v}$ le complété de $K$ par rapport à la valeur absolue $|\cdot|_{v}$, et par $\mathbb{C}_{v}$ le complété d'une clôture algébrique de $K_{v}$. La valeur absolue $|\cdot|_{v}$ sur $K$ s'étend de façon unique sur $\mathbb{C}_{v}$.

Pour toute place finie $\mathfrak{p} \in \Sigma_{f}$, on désigne par $\mathbf{m}_{\mathfrak{p}}$ la mesure de Haar sur $K_{\mathfrak{p}}$ normalisée de sorte que $\mathbf{m}_{\mathfrak{p}}\left(\theta_{\mathfrak{p}}\right)=1$, où $\theta_{\mathfrak{p}}$ est l'anneau des éléments $a \in K_{\mathfrak{p}}$ tels que $|a|_{\mathfrak{p}} \leqslant 1$. Pour toute $v \in \Sigma_{\infty, r}$, soit $\mathbf{m}_{v}$ la mesure de Lebesgue sur $K_{v}=\mathbb{R}$. Pour toute $w \in \Sigma_{\infty, c}$, soit $\mathbf{m}_{w}$ le double de la mesure de Lebesgue sur $K_{w}=\mathbb{C}$.

Soit $\mathbf{A}_{K}$ l'anneau topologique des adèles de $K$. On rappelle que $\mathbf{A}_{K}$ est le sousespace du produit $\prod_{v \in \Sigma_{f} \cup \Sigma_{\infty}} K_{v}$ des éléments $\alpha=\left(\alpha_{v}\right)_{v \in \Sigma_{f} \cup \Sigma_{\infty}}$ tels que, pour tout 
$\mathfrak{p} \in \Sigma_{f}$ en dehors d'un nombre fini, on ait $\alpha_{\mathfrak{p}} \in \vartheta_{\mathfrak{p}}$. Le produit des mesures $\mathbf{m}_{v}$ définit une mesure de Haar $\mathbf{m}$ sur $\mathbf{A}_{K}$. En outre, le corps $K$ se plonge diagonalement dans $\mathbf{A}_{K}$ comme un sous-anneau et l'espace quotient $\mathbf{A}_{K} / K$ est compact. Plus généralement, si $E$ est un espace vectoriel de rang fini $r$ sur $K$, quitte à choisir une base de $E$, on peut identifier $E \otimes \mathbf{A}_{K}$ à $\mathbf{A}_{K}^{r}$ et donc on obtient une mesure de Haar $\mathbf{m}_{E}$ sur $E \otimes \mathbf{A}_{K}$. D'après la formule de produit, cette mesure ne dépend pas du choix de la base de $E$. En outre, le covolume de $E$ dans $E \otimes_{K} \mathbf{A}_{K}$ pour la mesure $\mathbf{m}_{E}$, c'est-à-dire la mesure d'un domaine fondamental du quotient $\left(E \otimes_{K} \mathbf{A}_{K}\right) / E$ pour $\mathbf{m}_{E}$, est égale à $\left|D_{K}\right|^{\operatorname{rg}(E) / 2}$, où $D_{K}$ est le discriminant absolu de $K$.

\subsection{Filtrations des espaces vectoriels}

On présente dans cette section les propriétés élémentaires des $\mathbb{R}$-filtrations. Ce sont des objets intermédiaires à travers desquels on étudie les invariants arithmétiques. On fixe un corps $k$. Sauf mention au contraire, tous les espaces vectoriels sont supposés être sur $k$ et de rang fini.

\subsubsection{Définition et constructions}

DÉFInition 1.2.1. - Soit $V$ un espace vectoriel sur $k$. On appelle $\mathbb{R}$-filtration (ou simplement filtration) de $V$ toute famille $\mathcal{F}=\left(\mathcal{F}_{a} V\right)_{a \in \mathbb{R}}$ de sous-espaces vectoriels de $V$, soumise aux conditions suivantes :

1) $\mathcal{F}_{a} V \supset \mathcal{F}_{a^{\prime}} V$ pour tous les nombres réels $a$ et $a^{\prime}$ tels que $a \leqslant a^{\prime}$,

2) $\mathcal{F}_{a} V=0$ pour $a$ suffisamment positif,

3) $\mathcal{F}_{a} V=V$ pour $a$ suffisamment négatif,

4) la fonction $a \mapsto \operatorname{rg}\left(\mathcal{F}_{a} V\right)$ est continue à gauche sur $\mathbb{R}$.

Un espace vectoriel filtré est un espace vectoriel muni d'une filtration, c'est-à-dire un couple $(V, \mathcal{F})$ où $V$ est un espace vectoriel et $\mathcal{F}$ est une filtration de $V$.

REmarque 1.2.2. - Les conditions 2)-4) sont en fait respectivement la séparation, l'exhaustivité et la continuité à gauche de la filtration $\mathcal{F}$, que l'on supposera toujours dans cet article.

Une filtration $\mathcal{F}$ de $V$ est équivalente à la donnée d'un drapeau

$$
V=V_{0} \supsetneq V_{1} \supsetneq V_{2} \supsetneq \cdots \supsetneq V_{d}=0
$$

de $V$ et d'une suite strictement croissante $\left(a_{i}\right)_{0 \leqslant i<d}$. En effet, on construit d'une telle donnée une filtration de la forme

$$
\mathcal{F}_{a} V=\bigcup_{\substack{0 \leqslant i<d \\ a_{i} \geqslant a}} V_{i} .
$$


Réciproquement, étant donnée une filtration $\mathcal{F}$, la fonction $a \mapsto \operatorname{rg}\left(\mathcal{F}_{a} V\right)$ est décroissante, continue à gauche et prend valeurs dans l'ensemble fini $\{0, \ldots, \operatorname{rg} V\}$, donc elle est de la forme

$$
\operatorname{rg}\left(\mathcal{F}_{a} V\right)=\operatorname{rg} V \cdot \mathbb{1}_{]-\infty, a_{0}\right]}(a)+\sum_{i=1}^{d-1} r_{i} \mathbb{1}_{] a_{i-1}, a_{i}\right]}(a),
$$

où $a_{0}<\cdots<a_{d-1}$ et $0<r_{d-1}<\cdots<r_{1}<\operatorname{rg} V$. Soit $V_{i}=\mathcal{F}_{a_{i}} V$ pour $i \in$ $\{0, \ldots, d-1\}$ et soit $V_{d}=0$. On obtient alors un drapeau $V=V_{0} \supsetneq V_{1} \supsetneq \cdots \supsetneq V_{d}=0$ et une suite strictement croissante $\left(a_{i}\right)_{0 \leqslant i<d}$.

La position d'un vecteur général de $V$ dans la filtration $\mathcal{F}$ est donnée par la fonction $\lambda_{\mathscr{F}}: V \rightarrow \mathbb{R} \cup\{+\infty\}$ suivante $:$

$$
\lambda_{\mathscr{F}}(x):=\sup \left\{a \in \mathbb{R} \mid x \in \mathcal{F}_{a} V\right\} .
$$

Cette application prend valeurs dans l'ensemble $\left\{a_{0}, \ldots, a_{d-1}\right\} \cup\{+\infty\}$, et elle est finie sur $V \backslash\{0\}$. On note en outre

$$
\lambda_{\min }(V, \mathcal{F})=a_{0}=\min _{x \in V} \lambda_{\mathscr{F}}(x), \quad \lambda_{\max }(V, \mathcal{F})=a_{d-1}=\sup _{0 \neq x \in V} \lambda_{\mathscr{F}}(x) .
$$

Si $V$ est nul, alors par convention $\lambda_{\min }(V, \mathcal{F})=+\infty$ et $\lambda_{\max }(V, \mathcal{F})=-\infty$.

Proposition 1.2.3. - 1) Pour tout $u \in K^{\times}$et tout $x \in V, \lambda_{\mathscr{F}}(u x)=\lambda_{\mathscr{F}}(x)$.

2) $\lambda_{\mathscr{G}}(x) \geqslant b$ si et seulement si $x \in \mathcal{F}_{b} V$.

3) Pour tous les éléments $x$ et $y$ de $V, \lambda_{\mathcal{F}}(x+y) \geqslant \min \left(\lambda_{\mathscr{F}}(x), \lambda_{\mathscr{F}}(y)\right)$.

4) Si $x$ et $y$ sont deux éléments de $V$ tels que $\lambda_{\mathscr{F}}(x) \neq \lambda_{\mathscr{F}}(y)$, alors $x+y \neq 0$, et $\lambda_{\mathscr{F}}(x+y)=\min \left(\lambda_{\mathscr{F}}(x), \lambda_{\mathscr{F}}(y)\right)$.

Démonstration. - 1) Comme $u$ est inversible, $x \in \mathcal{F}_{a} V$ si et seulement si $u x \in \mathcal{F}_{a} V$, d'où $\left\{a \in \mathbb{R} \mid x \in \mathcal{F}_{a} V\right\}=\left\{a \in \mathbb{R} \mid u x \in \mathcal{F}_{a} V\right\}$.

2) Si $x \in \mathcal{F}_{b} V$, alors $b \in\left\{a \in \mathbb{R} \mid x \in \mathcal{F}_{a} V\right\}$. Donc $\lambda_{\mathscr{G}}(x) \geqslant b$. Réciproquement, si $\lambda_{\mathscr{F}}(x) \geqslant b$, alors $\lambda_{\mathscr{F}}(x)>b-\varepsilon$ quel que soit $\varepsilon>0$. On en déduit $x \in \mathcal{F}_{b-\varepsilon} V$ pour tout $\varepsilon>0$. Or la fonction $a \mapsto \operatorname{rg}\left(\mathcal{F}_{a} V\right)$ est continue à gauche, l'égalité $\mathcal{F}_{b-\varepsilon} V=\mathcal{F}_{b} V$ est vraie lorsque $\varepsilon$ est suffisamment petit. Donc $x \in \mathcal{F}_{b} V$.

3) Soient $a=\lambda_{\mathscr{F}}(x)$ et $b=\lambda_{\mathscr{F}}(y)$. D'après 2), on a $x \in \mathcal{F}_{a} V$ et $y \in \mathcal{F}_{b} V$. Il est anodin de supposer $a \geqslant b$. Alors $x \in \mathcal{F}_{b} V$ puisque $\mathcal{F}_{a} V \subset \mathcal{F}_{b} V$. On en déduit donc $x+y \in \mathcal{F}_{b} V$ et $\lambda_{\mathscr{F}}(x+y) \geqslant b$, compte tenu de 2).

4) Si $x+y=0$, alors $x=-y$. D'après 1$)$, on a $\lambda_{\mathscr{F}}(x)=\lambda_{\mathscr{F}}(y)$. Cela est absurde. Donc $x+y$ est non-nul. On peut supposer $\lambda_{\mathscr{F}}(x)<\lambda_{\mathscr{F}}(y)$. Pour tout $\left.a \in\right] \lambda_{\mathscr{F}}(x), \lambda_{\mathscr{F}}(y)[$, on a $y \in \mathcal{F}_{a} V$ mais $x \notin \mathcal{F}_{a} V$. Donc $x+y \notin \mathcal{F}_{a} V$ et $\lambda_{\mathscr{F}}(x+y)<a$. Comme $a$ est arbitraire, $\lambda_{\mathscr{F}}(x+y) \leqslant \lambda_{\mathscr{F}}(x)$. D'après 3$)$, on obtient l'égalité.

Étant données une application $k$-linéaire $f: V \rightarrow W$ d'espaces vectoriels et une filtration de l'un des espaces $V$ et $W$, sous certaine condition de $f$, on peut construire "naturellement" une filtration de l'autre espace. On présente dans la suite ces constructions en commençant par définir la compatibilité de $f$ aux filtrations. 
Soient $\mathcal{F}$ et $\mathscr{G}$ des filtrations de $V$ et de $W$ respectivement. On dit que l'application $f$ est compatible aux filtrations $(\mathcal{F}, \mathscr{G})$ si $f\left(\mathcal{F}_{a} V\right) \subset \mathscr{G}_{a} W$ quel que soit $a \in \mathbb{R}$, ou de façon équivalente, $\lambda_{\mathscr{G}}(f(x)) \geqslant \lambda_{\mathscr{F}}(x)$ pour tout $x \in V$.

La composition de deux applications $k$-linéaires compatibles aux filtrations est encore compatible aux filtrations. Donc les espaces vectoriels filtrés et les applications $k$-linéaires compatibles aux filtrations forment une catégorie que l'on notera $\mathbf{F i l}_{k}$.

Si $f: V \rightarrow W$ est injective et si $\mathscr{G}$ est une filtration de $W$, on désigne par $f^{*} \mathscr{G}$ la filtration de $V$ telle que $\left(f^{*} \mathscr{G}\right)_{a} V=f^{-1}\left(\mathscr{G}_{a} W\right)$ pour tout $a \in \mathbb{R}$, appelée la filtration induite (de $\mathscr{G}$ par $f$ ). Pour tout $x \in V$, on a $\lambda_{f * \mathscr{G}}(x)=\lambda_{\mathscr{G}}(f(x))$. Donc l'application $f$ est compatible aux filtrations $\left(f^{*} \mathscr{G}, \mathscr{G}\right)$.

Si $f$ est surjective et si $\mathcal{F}$ est une filtration de $V$, on définit une filtration notée $f_{*} \mathcal{F}$ de $W$ telle que $\left(f_{*} \mathcal{F}\right)_{a} W=f\left(\mathcal{F}_{a} V\right)$ pour tout $a \in \mathbb{R}$, appelée la filtration quotiente $\left(\operatorname{de} \mathcal{F}\right.$ par $f$ ). Pour tout $y \in W$, on a $\lambda_{f_{*}} \mathcal{F}(y)=\sup _{x \in f^{-1}(y)} \lambda_{\mathscr{F}}(x)$. L'application $f$ est compatible aux filtrations $\left(\mathcal{F}, f_{*} \mathcal{F}\right)$.

1.2.2. Mesure associée à une filtration. - Soit $V$ un espace vectoriel de rang $r$, où $r \in \mathbb{N}^{*}$. À chaque filtration de $V$ sera associée une mesure de probabilité borélienne sur $\mathbb{R}$. On montrera que cette mesure admet une additivité par rapport aux suites exactes courtes (d'espaces vectoriels filtrés).

Soit $\mathscr{M}_{1}$ l'ensemble des mesures de probabilité boréliennes sur $\mathbb{R}$. On définit une relation d'ordre $\succ \operatorname{sur} \mathscr{M}_{1}$ :

$\nu_{1} \succ \nu_{2}$ si et seulement si $\int_{\mathbb{R}} f \mathrm{~d} \nu_{1} \geqslant \int_{\mathbb{R}} f \mathrm{~d} \nu_{2}$ pour toute fonction croissante et bornée $f$ sur $\mathbb{R}$.

L'expression $\nu_{2} \prec \nu_{1}$ est aussi utilisée pour désigner la relation $\nu_{1} \succ \nu_{2}$.

Soit $\mathcal{F}$ une filtration de $V$. Si e $=\left(e_{1}, \ldots, e_{r}\right)$ est une base de $V$, on désigne par $\nu_{\mathscr{G}, \mathbf{e}}$ la mesure de probabilité

$$
\nu_{\mathcal{G}, \mathbf{e}}=\frac{1}{r} \sum_{i=1}^{r} \delta_{\lambda_{\mathscr{G}}\left(e_{i}\right)},
$$

qui est une combinaison linéaire de mesures de Dirac. Le sous-ensemble de $\mathscr{M}_{1}$ formé des mesures de la forme $\nu_{\mathscr{G}, \mathbf{e}}$ où e parcourt toutes les bases de $V$ admet un élément maximal $\nu_{\mathscr{G}}$ pour la relation d'ordre $\succ$, appelé la mesure associée à $\mathcal{F}$. En effet, la mesure $\nu_{\mathcal{F}, \mathbf{e}}$ est maximale si et seulement si la base e est compatible à la filtration $\mathcal{F}$, c'est-à-dire que \# $\left(\mathbf{e} \cap \mathcal{F}_{r} V\right)=\operatorname{rg}\left(\mathcal{F}_{r} V\right)$ quel que soit $r \in \mathbb{R}$.

Proposition 1.2.4. - Pour toute base e de $V$, il existe une matrice triangulaire $A$ dans $M_{r \times r}(k)$ dont la diagonale est $(1, \ldots, 1)$ et telle que Ae soit compatible à la filtration $\mathcal{F}$.

Démonstration. - C'est une conséquence de la décomposition de Bruhat pour le groupe linéaire général. Voir [16] 4.2.15 pour une preuve directe. 
Si la filtration $\mathcal{F}$ correspond au drapeau $V=V_{0} \supsetneq V_{1} \supsetneq V_{2} \supsetneq \cdots \supsetneq V_{d}=0$ et à la suite $\left(a_{i}\right)_{0 \leqslant i<d}$, alors la mesure $\nu_{\mathscr{G}}$ s'écrit comme

$$
\nu_{\mathscr{G}}=\sum_{i=0}^{d-1} \frac{\operatorname{rg} V_{i}-\operatorname{rg} V_{i+1}}{\operatorname{rg} V} \delta_{a_{i}},
$$

ou de façon équivalente, $\nu_{\mathscr{F}}$ est la dérivée d'ordre un au sens de distribution de la fonction $x \mapsto-\operatorname{rg}\left(\mathcal{F}_{x} V\right) / \operatorname{rg} V$. En effet, si $\mathbf{e}=\left(e_{1}, \ldots, e_{r}\right)$ est une base compatible à la filtration $\mathcal{F}$, alors le nombre des éléments $e_{j}$ tels que $\lambda_{\mathcal{F}}\left(e_{j}\right)=a_{i}$ est égal à $\operatorname{rg}\left(V_{i} / V_{i+1}\right)$. On déduit de la formule (4) que

$$
\lambda_{\min }(V, \mathcal{F})=\inf \left(\operatorname{supp} \nu_{\mathscr{F}}\right) \quad \text { et } \quad \lambda_{\max }(V, \mathcal{F})=\sup \left(\operatorname{supp} \nu_{\mathscr{F}}\right),
$$

où $\operatorname{supp} \nu_{\mathscr{G}}$ est le support de la mesure $\nu_{\mathscr{G}}$.

L'espace vectoriel nul admet une seule filtration. Pour simplifier les notations, on définit par convention la mesure associée à l'espace nul comme la mesure nulle. Attention : ce n'est plus une mesure de probabilité.

Bien que la catégorie $\mathbf{F i l}_{k}$ des espaces filtrés et des applications linéaires compatibles aux filtrations n'est guère une catégorie abélienne, les suites exactes courtes sont naturellement définies : on dit qu'une suite

$$
0 \longrightarrow\left(V^{\prime}, \mathcal{F}^{\prime}\right) \longrightarrow(V, \mathcal{F}) \longrightarrow\left(V^{\prime \prime}, \mathcal{F}^{\prime \prime}\right) \longrightarrow 0
$$

de morphismes dans $\mathbf{F i l}_{k}$ est exacte si

1) la suite $0 \longrightarrow V^{\prime} \longrightarrow V \longrightarrow V^{\prime \prime} \longrightarrow 0$ est exacte,

2) la filtration $\mathcal{F}^{\prime}$ est la filtration induite,

3) la filtration $\mathcal{F}^{\prime \prime}$ est la filtration quotiente.

Proposition 1.2.5. - Si $0 \longrightarrow\left(V^{\prime}, \mathcal{F}^{\prime}\right) \longrightarrow(V, \mathcal{F}) \longrightarrow\left(V^{\prime \prime}, \mathcal{F}^{\prime \prime}\right) \longrightarrow 0$ est une suite exacte dans $\mathbf{F i l} k$, alors

$$
\operatorname{rg} V \cdot \nu_{\mathscr{G}}=\operatorname{rg} V^{\prime} \cdot \nu_{\mathscr{F}^{\prime}}+\operatorname{rg} V^{\prime \prime} \cdot \nu_{\mathscr{G}^{\prime \prime}}
$$

Démonstration. - La suite $0 \longrightarrow\left(V^{\prime}, \mathcal{F}^{\prime}\right) \longrightarrow(V, \mathcal{F}) \longrightarrow\left(V^{\prime \prime}, \mathcal{F}^{\prime \prime}\right) \longrightarrow 0$ est exacte si et seulement si, pour tout $r \in \mathbb{R}$, la suite

$$
0 \longrightarrow \mathcal{F}_{r}^{\prime} V^{\prime} \longrightarrow \mathcal{F}_{r} V \longrightarrow \mathcal{F}_{r}^{\prime \prime} V^{\prime \prime} \longrightarrow 0
$$

d'homomorphismes $k$-linéaires est exacte. On obtient ainsi l'égalité $\operatorname{rg}\left(\mathcal{F}_{r} V\right)=$ $\operatorname{rg}\left(\mathcal{F}_{r}^{\prime} V^{\prime}\right)+\operatorname{rg}\left(\mathcal{F}_{r}^{\prime \prime} V^{\prime \prime}\right)$ et puis l'égalité (5) en prenant la dérivée au sens de distribution. 
1.2.3. Extension de base. - Si $k^{\prime} / k$ est une extension de corps, alors toute filtration $\mathcal{F}$ de $V$ induit naturellement une filtration $\widetilde{\mathcal{F}}$ de $V_{k^{\prime}}:=V \otimes_{k} k^{\prime}$ telle que, pour tout $r \in \mathbb{R}, \widetilde{\mathcal{F}}_{r} V_{k^{\prime}}=\left(\mathcal{F}_{r} V\right) \otimes_{k} k^{\prime}$. Cette construction est fonctorielle, c'est-à-dire que l'application $(V, \mathcal{F}) \mapsto\left(V_{k^{\prime}}, \widetilde{\mathcal{F}}\right)$ définit en fait un foncteur de $\mathbf{F i l}{ }_{k}$ vers $\mathbf{F i l} k_{k^{\prime}}$. Ce foncteur est exact dans le sens suivant : il envoie une suite exacte courte dans $\mathbf{F i l}{ }_{k}$ vers une suite exacte courte dans $\mathbf{F i l}_{k^{\prime}}$. En outre, ce foncteur préserve la mesure associée, plus précisément, on a $\nu_{\mathscr{G}}=\nu_{\widetilde{\mathscr{T}}}$.

1.2.4. Opérations sur les mesures. - On introduit deux sortes d'opérations sur l'espace de mesures $\mathscr{M}_{1}$. Pour tout $a \in \mathbb{R}$, soit $\varphi_{a}: \mathbb{R} \rightarrow \mathbb{R}$ l'application de translation qui envoie $x$ en $x+a$. Elle induit un automorphisme $\tau_{a}: \mathscr{M}_{1} \rightarrow \mathscr{M}_{1}$ qui envoie une mesure de probabilité $\nu$ en son image directe par $\varphi_{a}$. Autrement dit, pour toute $\nu \in \mathscr{M}_{1}$ et toute fonction borélienne bornée $h$ sur $\mathbb{R}$, on a

$$
\int_{\mathbb{R}} h(x) \tau_{a} \nu(\mathrm{d} x)=\int_{\mathbb{R}} h(x+a) \nu(\mathrm{d} x)
$$

Par définition, on a $\tau_{a+a^{\prime}}=\tau_{a} \circ \tau_{a^{\prime}}$. Pour tout $\varepsilon>0$, soit $\gamma_{\varepsilon}: \mathbb{R} \rightarrow \mathbb{R}$ l'application de dilatation qui envoie $x$ en $\varepsilon x$. Elle induit par image directe un automorphisme $T_{\varepsilon}: \mathscr{M}_{1} \rightarrow \mathscr{M}_{1}$. Pour toute $\nu \in \mathscr{M}_{1}$ et toute fonction borélienne bornée $h$ sur $\mathbb{R}$, on a

$$
\int_{\mathbb{R}} h(x) T_{\varepsilon} \nu(\mathrm{d} x)=\int_{\mathbb{R}} h(\varepsilon x) \nu(\mathrm{d} x) .
$$

On a $T_{\varepsilon \varepsilon^{\prime}}=T_{\varepsilon} \circ T_{\varepsilon^{\prime}}$. En outre, les automorphismes $\tau_{a}$ et $T_{\varepsilon}$ préservent la relation d'ordre $\succ$.

Étant donnés deux espaces filtrés et une bijection $k$-linéaire des espaces vectoriels sous-jacents, le lemme au-dessous compare les filtrations de la source et du but :

Lemme 1.2.6. - Soient $(V, \mathcal{F})$ et $\left(V^{\prime}, \mathcal{F}^{\prime}\right)$ deux espaces vectoriels filtrés, $\varphi: V \rightarrow V^{\prime}$ un isomorphisme de $k$-espaces vectoriels et $c$ un nombre réel. Si, pour tout $x \in V$, on $a \lambda_{\mathscr{F}}(x) \leqslant \lambda_{\mathscr{F}^{\prime}}(\varphi(x))+c$, alors $\tau_{c} \nu_{\mathscr{F}^{\prime}} \succ \nu_{\mathscr{G}}$.

Démonstration. - Soit $\mathbf{e}=\left(e_{i}\right)_{1 \leqslant i \leqslant n}$ une base de $V$ qui est compatible à la filtration $\mathcal{F}$. Alors $\mathbf{e}^{\prime}=\left(\varphi\left(e_{i}\right)\right)_{1 \leqslant i \leqslant n}$ est une base de $V^{\prime}$, et donc

$$
\tau_{c} \nu_{\mathscr{F}^{\prime}} \succ \tau_{c} \nu_{\mathscr{F}^{\prime}, \mathbf{e}^{\prime}}=\frac{1}{n} \sum_{i=1}^{n} \delta_{\lambda_{\mathscr{F}^{\prime}}\left(\varphi\left(e_{i}\right)\right)+c} \succ \frac{1}{n} \sum_{i=1}^{n} \delta_{\lambda_{\mathscr{F}}\left(e_{i}\right)}=\nu_{\mathscr{G}} .
$$

1.2.5. Polygone associé à une mesure. - On désigne par $\mathscr{C}_{0}$ l'ensemble des fonctions concaves définies sur [0,1] qui valent 0 en l'origine. Soit $\mathscr{M}_{1, c}$ l'ensemble des mesures de probabilité boréliennes à support compact sur $\mathbb{R}$. On définit au-dessous une application $\mathscr{P}: \mathscr{M}_{1, c} \rightarrow \mathscr{C}_{0}$. Cette application sera utilisée plus loin pour retrouver les polygones de Harder-Narasimhan.

Soient $\nu \in \mathscr{M}_{1, c}$ et $F_{\nu}: \mathbb{R} \rightarrow[0,1]$ la fonction définie par $F_{\nu}(x)=\nu(] x,+\infty[)$. On désigne par $\mathscr{P}(\nu):[0,1] \rightarrow \mathbb{R}$ la transformée de Legendre (cf. [32] II §2.2) de la fonction concave $x \mapsto \int_{0}^{x} F_{\nu}(y) d y$. 
Voici une forme explicite de la fonction $\mathscr{P}(\nu)$. Soit $F_{\nu}^{*}:[0,1[\rightarrow \mathbb{R}$ la fonction telle que $F_{\nu}^{*}(t)=\sup \left\{x \mid F_{\nu}(x)>t\right\}$. Comme $\nu$ est à support compact, $F_{\nu}^{*}$ est une fonction décroissante, continue à droite et bornée. En outre, pour tout $t \in[0,1[$ et tout $y \in \mathbb{R}, F_{\nu}(y)>t$ si et seulement si $y<F_{\nu}^{*}(t)$. La fonction $\mathscr{P}(\nu)$ envoie $t \in[0,1]$ en $\int_{[0, t[} F_{\nu}^{*}(s) d s$.

La proposition suivante montre que l'application $\mathscr{P}$ préserve la relation d'ordre "२".

Proposition 1.2.7. - Si $\nu_{1}$ et $\nu_{2}$ sont deux mesures dans $\mathscr{M}_{1, c}$ telles que $\nu_{1} \prec \nu_{2}$, alors $\mathscr{P}\left(\nu_{1}\right)(t) \leqslant \mathscr{P}\left(\nu_{2}\right)(t)$ quel que soit $t \in[0,1]$.

Démonstration. - Par définition, $F_{\nu_{1}}(x) \leqslant F_{\nu_{2}}(x)$ quel que soit $x \in \mathbb{R}$. Par conséquent, $F_{\nu_{1}}^{*} \leqslant F_{\nu_{2}}^{*}$ et donc $\mathscr{P}\left(\nu_{1}\right) \leqslant \mathscr{P}\left(\nu_{2}\right)$.

L'espace $\mathscr{M}_{1, c}$ est invariant par les opérateurs $\tau_{a}$ et $T_{\varepsilon}$. De plus, on a les égalités :

$$
\mathscr{P}\left(\tau_{a} \nu\right)(t)=\mathscr{P}(\nu)(t)+a t, \quad \mathscr{P}\left(T_{\varepsilon} \nu\right)=\varepsilon \mathscr{P}(\nu) .
$$

Si $\nu$ est une combinaison linéaire de mesures de Dirac, alors $F_{\nu}^{*}$ est une fonction d'escalier. Par conséquent, la fonction $\mathscr{P}(\nu)$ est linéaire par morceaux. Une telle fonction est appelée un polygone sur $[0,1]$. En particulier, si $(V, \mathcal{F})$ est un espace vectoriel filtré, alors $\mathscr{P}\left(\nu_{\mathscr{G}}\right)$ est un polygone.

DÉFInition 1.2.8. - La fonction $\mathscr{P}\left(\nu_{\mathcal{G}}\right)$ est appelée le polygone associé à $\mathcal{F}$.

On rappelle qu'une suite de mesures boréliennes $\left(\nu_{n}\right)_{n \geqslant 1}$ sur $\mathbb{R}$ converge vaguement vers une mesure borélienne $\nu$ si et seulement si, pour toute fonction $f$ continue et à support compact sur $\mathbb{R}$, la suite des intégrales $\left(\int_{\mathbb{R}} f \mathrm{~d} \nu_{n}\right)_{n \geqslant 1}$ converge vers $\int_{\mathbb{R}} f \mathrm{~d} \nu$. La convergence vague des mesures implique la convergence uniforme des polygones.

Proposition 1.2.9. - Soit $\left(\nu_{n}\right)_{n \geqslant 1}$ une suite de mesures dans $\mathscr{M}_{1, c}$. On suppose que les supports des mesures $\nu_{n}$ sont uniformément bornés et que la suite de mesures $\left(\nu_{n}\right)_{n \geqslant 1}$ converge vaguement vers $\nu$. Alors la mesure limite $\nu$ est aussi dans $\mathscr{M}_{1, c}$; de plus, la suite de fonctions $\left(\mathscr{P}\left(\nu_{n}\right)\right)_{n \geqslant 1}$ converge uniformément sur $[0,1]$ vers $\mathscr{P}(\nu)$.

Démonstration. - Soit $I=[A, B]$ un intervalle fermé et borné tel que supp $\nu_{n} \subset I$ pour tout $n$. Comme les supports des $\nu_{n}$ sont tous contenus dans $I$, il en est de même du support de $\nu$. Si $f$ est une fonction à support compact et à valeurs dans $[0,1]$ telle que $\left.f\right|_{I} \equiv 1$, alors $\int_{\mathbb{R}} f \mathrm{~d} \nu_{n}=1$ pour tout $n$. On en déduit $\int_{\mathbb{R}} f \mathrm{~d} \nu=1$. Donc $\nu$ est une mesure de probabilité. Soit $Z$ l'ensemble des $x \in \mathbb{R}$ tel que $\nu(\{x\}) \neq 0$, qui est un ensemble dénombrable. Alors la suite $F_{\nu_{n}}$ converge simplement vers $F_{\nu}$ sur $\mathbb{R} \backslash Z$ d'après [12] IV $\S 5 \mathrm{n}^{\circ} 12$ Proposition 22.

Si $t \in] 0,1\left[\right.$ et si $y=F_{\nu}^{*}(t)$, alors il existe une suite strictement croissante $\left(x_{m}\right)_{m \geqslant 1} \subset$ $\mathbb{R} \backslash Z$ qui converge vers $y$. Comme $x_{m}<y$, on a $F_{\nu}\left(x_{m}\right)>t$. Comme $x_{m} \notin Z$, il existe $N(m) \in \mathbb{N}$ tel que $F_{\nu_{n}}\left(x_{m}\right)>t$, ou de façon équivalente, $x_{m}<F_{\nu_{n}}^{*}(t)$ pour tout $n>N(m)$. Cela implique $\liminf _{n \rightarrow+\infty} F_{\nu_{n}}^{*}(t) \geqslant F_{\nu}^{*}(t)$. 
Pour tout entier $n \geqslant 1$, soit $U_{n}$ l'ensemble des $t \in\left[0,1\left[\right.\right.$ tels que $F_{\nu_{n}}^{-1}(\{t\})$ ait un point intérieur. C'est un sous-ensemble dénombrable de [0,1[. Soient $U^{\prime \prime}$ l'ensemble des $t \in\left[0,1\left[\right.\right.$ tels que $F_{\nu}^{-1}(\{t\})$ ait un point intérieur, et $U$ l'union de $U^{\prime \prime}$ et de tous les $U_{n}^{\prime}$. L'ensemble $U$ est dénombrable. Si $t \in\left[0,1\left[\backslash U\right.\right.$ et si $y=F_{\nu}^{*}(t)$, alors il existe une suite strictement décroissante $\left(x_{m}\right)_{m \geqslant 1} \subset \mathbb{R} \backslash Z$ qui converge vers $y$. Comme $t \notin U^{\prime \prime}$, on obtient $F_{\nu}\left(x_{m}\right)<t$. Donc il existe $N(m) \in \mathbb{N}$ tel que $F_{\nu_{n}}\left(x_{m}\right)<t$ et alors $x_{m} \geqslant F_{\nu_{n}}^{*}(t)$ pour tout $n>N(m)$. On en déduit $\limsup _{n \rightarrow+\infty} F_{\nu_{n}}^{*}(t) \leqslant F_{\nu}^{*}(t)$.

D'après les arguments comme ci-dessus, il existe un sous-ensemble dénombrable $U$ de $\left[0,1\left[\right.\right.$ tel que $\left(F_{\nu_{n}}^{*}\right)_{n \geqslant 1}$ converge simplement vers $F_{\nu}^{*}$ sur [0,1[\U. De plus, les fonctions $F_{\nu_{n}}^{*}$ sont uniformément bornées. Donc le théorème de convergence dominée montre que

$$
\left|\int_{[0, t[} F_{\nu_{n}}^{*}(s) \mathrm{d} s-\int_{[0, t[} F_{\nu}^{*}(s) \mathrm{d} s\right| \leqslant \int_{0}^{1}\left|F_{\nu_{n}}^{*}(s)-F_{\nu}^{*}(s)\right| \mathrm{d} s
$$

convergent vers 0 quand $n$ tend vers l'infini.

On présente enfin quelques faits concernant la convergence vague des mesures que l'on utilisera plus loin dans le chapitre 3.

LEMME 1.2.10. - Si $f$ est une fonction continue et à support compact sur $\mathbb{R}$, alors

$$
\lim _{\varepsilon \rightarrow 0}\left\|f \circ \varphi_{\varepsilon}-f\right\|_{\sup }=0, \quad \lim _{\varepsilon \rightarrow 0}\left\|f \circ \gamma_{1+\varepsilon}-f\right\|_{\text {sup }}=0 .
$$

Démonstration. - Comme $f$ est à support compact, elle est uniformément continue. La première égalité est donc vraie.

On suppose que $\operatorname{supp}(f) \subset[-M, M]$, où $M>0$. Pour tout $\varepsilon \in[-1 / 2,1 / 2]$, on a

$$
\left\|f \circ \gamma_{1+\varepsilon}-f\right\|_{\text {sup }}=\sup _{-2 M \leqslant x \leqslant 2 M}|f(x+\varepsilon x)-f(x)| .
$$

Comme $f$ est uniformément continue,

$$
\lim _{\varepsilon \rightarrow 0} \sup _{-2 M \leqslant x \leqslant 2 M}|f(x+\varepsilon x)-f(x)|=0 .
$$

On en déduit donc $\lim _{\varepsilon \rightarrow 0}\left\|f \circ \gamma_{1+\varepsilon}-f\right\|_{\text {sup }}=0$.

LEMME 1.2.11. - Soient $\left(\nu_{n}\right)_{n \geqslant 1}$ une suite dans $\mathscr{M}_{1}$ et $\nu$ une mesure borélienne sur $\mathbb{R}$. Soit $\left(a_{n}\right)_{n \geqslant 1}$ une suite dans $]-1,+\infty[$ qui converge vers 0 . Les conditions suivantes sont équivalentes :

1) la suite $\left(\nu_{n}\right)_{n \geqslant 1}$ converge vaguement vers $\nu$;

2) la suite $\left(T_{1+a_{n}} \nu_{n}\right)_{n \geqslant 1}$ converge vaguement vers $\nu$;

3) la suite $\left(\tau_{a_{n}} \nu_{n}\right)_{n \geqslant 1}$ converge vaguement vers $\nu$.

Démonstration. - Comme $\tau_{a_{n}}^{-1}=\tau_{-a_{n}}$ et comme $T_{1+a_{n}}^{-1}=T_{\left(1+a_{n}\right)^{-1}}=T_{1-\frac{a_{n}}{1+a_{n}}}$, il suffit de vérifier " 1$) \Longrightarrow 2$ )" et " 1 ) $\Longrightarrow 3$ )", qui sont des conséquences immédiates du lemme 1.2.10. 
Lemme 1.2.12. - Soient $\left(\nu_{n}^{\prime}\right)_{n \geqslant 1},\left(\nu_{n}\right)_{n \geqslant 1}$ et $\left(\nu_{n}^{\prime \prime}\right)_{n \geqslant 1}$ trois suites de mesures dans $\mathscr{M}_{1, c}$ telles que $\nu_{n}^{\prime} \prec \nu_{n} \prec \nu_{n}^{\prime \prime}$ quel que soit $n \geqslant 1$. On suppose que les supports des mesures $\nu_{n}^{\prime}, \nu_{n}$ et $\nu_{n}^{\prime \prime}$ sont uniformément bornés et que les deux suites $\left(\nu_{n}^{\prime}\right)_{n \geqslant 1}$ et $\left(\nu_{n}^{\prime \prime}\right)_{n \geqslant 1}$ convergent vaguement vers la même limite $\nu$. Alors la suite $\left(\nu_{n}\right)_{n \geqslant 1}$ converge vaguement aussi vers $\nu$.

Démonstration. - Comme les mesures que l'on considère sont à supports uniformément bornés, pour toute fonction continue $f$ sur $\mathbb{R}$, on a

$$
\lim _{n \rightarrow+\infty} \int_{\mathbb{R}} f \mathrm{~d} \nu_{n}^{\prime}=\lim _{n \rightarrow+\infty} \int_{\mathbb{R}} f \mathrm{~d} \nu_{n}^{\prime \prime}=\int_{\mathbb{R}} f \mathrm{~d} \nu .
$$

Or, par l'hypothèse, si $f$ est croissante et bornée,

$$
\int_{\mathbb{R}} f \mathrm{~d} \nu_{n}^{\prime} \leqslant \int_{\mathbb{R}} f \mathrm{~d} \nu_{n} \leqslant \int_{\mathbb{R}} f \mathrm{~d} \nu_{n}^{\prime \prime} .
$$

Ainsi $\lim _{n \rightarrow+\infty} \int_{\mathbb{R}} f \mathrm{~d} \nu_{n}=\int_{\mathbb{R}} f \mathrm{~d} \nu$ pour une telle fonction $f$. Ensuite, comme toute fonction lisse et à support compact peut s'écrire comme la différence de deux fonctions continues croissantes et bornées, l'égalité $\lim _{n \rightarrow \infty} \int_{\mathbb{R}} f \mathrm{~d} \nu_{n}=\int_{\mathbb{R}} f \mathrm{~d} \nu$ est vraie pour toute fonction $f$ lisse et à support compact. Enfin, comme l'espace $C_{0}^{\infty}(\mathbb{R})$ des fonctions lisses et à support compact est dense dans l'espace $C_{c}(\mathbb{R})$ des fonctions continues et à support compact pour la topologie définie par la norme $\|\cdot\|_{\text {sup }}$, on obtient que l'égalité $\lim _{n \rightarrow \infty} \int_{\mathbb{R}} f \mathrm{~d} \nu_{n}=\int_{\mathbb{R}} f \mathrm{~d} \nu$ est vraie pour toute $f \in C_{c}(\mathbb{R})$. Donc la suite de mesures $\left(\nu_{n}\right)_{n \geqslant 1}$ converge vaguement vers $\nu$.

1.2.6. Conventions. - Soit $V$ un espace vectoriel sur $k$ muni d'une $\mathbb{R}$-filtration $\mathcal{F}$. Dans la suite, s'il y a aucune ambiguïté sur la filtration $\mathcal{F}$, on utilisera aussi l'expression $\lambda_{V}$ ou simplement $\lambda$ pour désigner la fonction $\lambda_{\mathscr{F}}$, et on utilise le symbole $\nu_{V}$ pour désigner la mesure associée à la filtration $\mathcal{F}$. De façon similaire, les expressions $\lambda_{\min }(V)$ et $\lambda_{\max }(V)$ désignent $\lambda_{\min }(V, \mathcal{F})$ et $\lambda_{\max }(V, \mathcal{F})$, respectivement.

\subsection{Suites presque sous(sur)-additives}

Le but de cette article est de montrer la convergence d'une suite de certains invariants arithmétiques normalisés. Une méthode très classique pour montrer la convergence d'une suite normalisée est le lemme de Fekete (voir [21] page 233 pour un cas particulier). Étant donnée une suite $\left(a_{n}\right)_{n \geqslant 1}$ de nombres réels, si cette suite est sous-additive (à savoir, $a_{n+m} \leqslant a_{n}+a_{m}$ quel que soit $(m, n) \in \mathbb{Z}_{>0}^{2}$ ), alors le lemme de Fekete affirme que la limite de la suite normalisée $\left(a_{n} / n\right)_{n \geqslant 1}$ existe dans $\mathbb{R} \cup\{-\infty\}$. La preuve de ce résultat est un exercice facile.

Cependant, le lemme de Fekete - sous sa forme primitive - ne suffit pas pour notre application arithmétique. En effet, il s'agit des suites qui sont seulement suradditives à des termes d'erreur près. Par conséquent, il faut développer des critères de convergence plus généraux. Dans la suite, on présentera deux telles généralisations dont la première (la proposition 1.3.1) concerne une suite multi-sous-additive à une 
erreur strictement sous-linéaire près, et la seconde (la proposition 1.3.5) traite des suites presque sous-additives en demandant une condition de croissance lente plus forte sur les termes d'erreur. Dans les corollaires 1.3.2, 1.3.3 et 1.3.6 on développera les variantes sur-additives que l'on utilisera plus loin.

Proposition 1.3.1. - Soient $\left(a_{n}\right)_{n \geqslant 1}$ une suite dans $\left[0,+\infty\left[\right.\right.$ et $f: \mathbb{Z}_{\geqslant 0} \rightarrow \mathbb{R}$ une application telle que $\lim _{n \rightarrow \infty} f(n) / n=0$. S'il existe un entier $n_{0} \geqslant 0$ tel que, pour toute famille finie $\left(n_{i}\right)_{1 \leqslant i \leqslant r}$ d'entiers supérieurs ou égaux à $n_{0}$, on ait $a_{n_{1}+\cdots+n_{r}} \leqslant$ $a_{n_{1}}+\cdots+a_{n_{r}}+f\left(n_{1}\right)+\cdots+f\left(n_{r}\right)$, alors la suite $\left(a_{n} / n\right)_{n \geqslant 1}$ admet une limite dans $[0,+\infty[$.

Démonstration. - Si $n$ et $p$ sont deux entiers dans $\mathbb{Z}_{>n_{0}}$ et si $l \in\{n, n+1, \ldots, 2 n-1\}$, alors

$$
\begin{aligned}
\frac{a_{p n+l}}{p n+l} & \leqslant \frac{p a_{n}+a_{l}}{p n+l}+\frac{p f(n)+f(l)}{p n+l} \leqslant \frac{a_{n}}{n}+\frac{a_{l}}{p n}+\frac{p f(n)+f(l)}{p n+l} \\
& \leqslant \frac{a_{n}}{n}+\frac{a_{l}}{p n}+\frac{|f(n)|}{n}+\frac{|f(l)|}{p n} .
\end{aligned}
$$

Comme

$$
\lim _{p \rightarrow \infty} \frac{\max _{n \leqslant i<2 n} a_{i}}{p n}+\frac{\max _{n \leqslant i<2 n}|f(i)|}{p n}=0,
$$

on obtient que, pour tout entier $n>0$,

$$
\limsup _{m \rightarrow \infty} \frac{a_{m}}{m} \leqslant \frac{a_{n}}{n}+\frac{|f(n)|}{n}
$$

d'où

$$
\limsup _{m \rightarrow \infty} \frac{a_{m}}{m} \leqslant \liminf _{n \rightarrow \infty}\left(\frac{a_{n}}{n}+\frac{|f(n)|}{n}\right) \leqslant \liminf _{n \rightarrow \infty} \frac{a_{n}}{n}+\limsup _{n \rightarrow \infty} \frac{|f(n)|}{n}=\liminf _{n \rightarrow \infty} \frac{a_{n}}{n} .
$$

Par conséquent, la suite $\left(a_{n} / n\right)_{n \geqslant 1}$ a une limite, qui est clairement positive, et est finie d'après (7).

Corollaire 1.3.2. - Soient $\left(a_{n}\right)_{n \geqslant 1}$ une suite de nombres réels et $f: \mathbb{Z}_{\geqslant 0} \rightarrow \mathbb{R}$ une application telle que $\lim _{n \rightarrow \infty} f(n) / n=0$. Si les deux conditions suivantes sont vérifiées:

1) il existe un entier $n_{0} \geqslant 0$ tel que, pour toute famille $\left(n_{i}\right)_{1 \leqslant i \leqslant r}$ d'entiers supérieurs ou égaux à $n_{0}$, on ait $a_{n_{1}+\cdots+n_{r}} \geqslant a_{n_{1}}+\cdots+a_{n_{r}}-f\left(n_{1}\right)-\cdots-f\left(n_{r}\right)$,

2) il existe une constante $\alpha>0$ telle que, pour tout entier $n \geqslant 1$, on ait $a_{n} \leqslant \alpha n$, alors la suite $\left(a_{n} / n\right)_{n \geqslant 1}$ admet une limite dans $\mathbb{R}$. 
Démonstration. - On considère la suite formée des nombres réels de la forme $b_{n}=$ $\alpha n-a_{n}$. C'est une suite positive. Si $n_{1}, \ldots, n_{r}$ sont des entiers supérieurs ou égaux à $n_{0}$ et si $n=n_{1}+\cdots+n_{r}$, alors

$$
\begin{aligned}
& b_{n}=\alpha n-a_{n}=\alpha \sum_{i=1}^{r} n_{i}-a_{n} \leqslant \alpha \sum_{i=1}^{r} n_{i}-\sum_{i=1}^{r}\left(a_{n_{i}}-f\left(n_{i}\right)\right) \\
= & \sum_{i=1}^{r}\left(\alpha n_{i}-a_{n_{i}}+f\left(n_{i}\right)\right)=b_{n_{1}}+\cdots+b_{n_{r}}+f\left(n_{1}\right)+\cdots+f\left(n_{r}\right) .
\end{aligned}
$$

D'après la proposition 1.3.1, la suite $\left(b_{n} / n\right)_{n \geqslant 1}$ admet une limite dans $\mathbb{R}$. Comme $b_{n} / n=\alpha-a_{n} / n$, la suite $\left(a_{n} / n\right)_{n \geqslant 1}$ a aussi une limite dans $\mathbb{R}$.

Corollaire 1.3.3. - Soient $\left(a_{n}\right)_{n \geqslant 1}$ une suite de nombres réels et $c_{1}, c_{2}$ deux constantes positives telles que:

1) pour tout couple $(m, n)$ d'entiers suffisamment grands, $a_{m}+a_{n} \leqslant a_{m+n}+c_{1}$,

2) pour tout entier $n \geqslant 1, a_{n} \leqslant c_{2} n$,

alors la suite $\left(a_{n} / n\right)_{n \geqslant 1}$ admet une limite dans $\mathbb{R}$.

Démonstration. - Il suffit d'appliquer le corollaire 1.3.2 à la fonction constante $f$ qui prend valeur $c_{1}$.

LEMME 1.3.4. - Si $f: \mathbb{Z}_{\geqslant 0} \rightarrow\left[0,+\infty\left[\right.\right.$ est une application telle que $\sum_{\alpha \geqslant 1} f\left(2^{\alpha}\right) / 2^{\alpha}<$ $+\infty$, alors $\lim _{\alpha \rightarrow+\infty} 2^{-\alpha} \sum_{i=0}^{\alpha} f\left(2^{i}\right)=0$.

Démonstration. - Pour tout entier $\alpha \geqslant 0$, soit $S_{\alpha}=\sum_{i \geqslant \alpha} f\left(2^{i}\right) / 2^{i}$. Par la sommation d'Abel, on a

$$
\sum_{i=0}^{\alpha} f\left(2^{i}\right)=\sum_{i=0}^{\alpha}\left(S_{i}-S_{i+1}\right) 2^{i}=S_{0}-S_{\alpha+1} 2^{\alpha}+\sum_{i=1}^{\alpha} S_{i} 2^{i-1}
$$

Comme $\lim _{\alpha \rightarrow+\infty} S_{\alpha}=0$, le terme $2^{-\alpha} \sum_{i=1}^{\alpha} S_{i} 2^{i-1}$ converge vers 0 lorsque $\alpha \rightarrow+\infty$. Cela montre que $\lim _{\alpha \rightarrow+\infty} 2^{-\alpha} \sum_{i=0}^{\alpha} f\left(2^{i}\right)=0$.

Proposition 1.3.5. - Soient $\left(b_{n}\right)_{n \geqslant 1}$ une suite de nombres positifs et $f: \mathbb{Z}_{\geqslant 0} \rightarrow$ $\left[0,+\infty\left[\right.\right.$ une application croissante telle que $\sum_{\alpha \geqslant 0} f\left(2^{\alpha}\right) / 2^{\alpha}<+\infty$. S'il existe un entier $n_{0}>0$ tel que, pour tout couple $(m, n)$ d'entiers dans $\mathbb{Z}_{\geqslant n_{0}}$, on ait $b_{n+m} \leqslant b_{m}+b_{n}+$ $f(m)+f(n)$, alors la suite $\left(b_{n} / n\right)_{n \geqslant 1}$ admet une limite dans $[0,+\infty[$. 
Démonstration. - On considère d'abord le cas où $n_{0}=1$. Comme $f$ est une fonction croissante, on obtient que, pour tout $(m, n) \in \mathbb{Z}_{>0}^{2}$,

$$
b_{m+n} \leqslant b_{n}+b_{m}+2 f(m+n) .
$$

Pour tout entier $\alpha \geqslant 0$, soit $S_{\alpha}=\sum_{i \geqslant \alpha} f\left(2^{i}\right) / 2^{i}$. D'après l'hypothèse, on a $\lim _{\alpha \rightarrow+\infty} S_{\alpha}=$ 0 . Si $n \in \mathbb{Z}_{>0}$ et $\beta \in \mathbb{N}$ sont tels que $2^{\beta} \leqslant n<2^{\beta+1}$, alors, pour tout $\alpha \in \mathbb{N}$, on a

$$
b_{2^{\alpha} n} \leqslant 2^{\alpha} b_{n}+\sum_{i=1}^{\alpha} 2^{\alpha+1-i} f\left(2^{i-1} n\right) \leqslant 2^{\alpha} b_{n}+\sum_{i=1}^{\alpha} 2^{\alpha+1-i} f\left(2^{\beta+i}\right) .
$$

Si $p=\sum_{i=0}^{k} \epsilon_{i} 2^{i}$, où $\epsilon_{i} \in\{0,1\}$ pour tout $i \in\{0,1, \ldots, k-1\}$ et où $\epsilon_{k}=1$, et si $r \in\{0,1, \ldots, n-1\}$, d'après $(8)$, on a l'inégalité suivante:

$$
b_{n p+r} \leqslant b_{n p}+b_{r}+2 f(n p+r) \leqslant \sum_{i=0}^{k} \epsilon_{i} b_{2^{i} n}+b_{r}+2 \sum_{i=0}^{k} \epsilon_{i} f\left(\sum_{j=0}^{i} \epsilon_{j} 2^{j} n\right)+2 f(n p+r)
$$

Compte tenu de l'inégalité (9), on obtient

$$
\begin{gathered}
b_{n p+r} \leqslant \sum_{i=0}^{k} \epsilon_{i} 2^{i} b_{n}+b_{r}+\sum_{i=1}^{k} \epsilon_{i} \sum_{j=1}^{i} 2^{i+1-j} f\left(2^{\beta+j}\right) \\
+2 \sum_{i=0}^{k} \epsilon_{i} f\left(2^{i+\beta+2}\right)+2 f\left(2^{k+\beta+2}\right) .
\end{gathered}
$$

Par conséquent,

$$
\begin{aligned}
& \frac{b_{n p+r}}{n p+r} \leqslant \frac{p b_{n}}{n p+r}+\frac{b_{r}}{n p+r}+2^{-k-\beta} \sum_{i=1}^{k} \sum_{j=1}^{i} 2^{i+1-j} f\left(2^{\beta+j}\right) \\
& \quad+2^{-k-\beta+1} \sum_{i=0}^{k} f\left(2^{i+\beta+2}\right)+2^{-k-\beta+1} f\left(2^{k+\beta+2}\right) .
\end{aligned}
$$

Comme

$$
\begin{aligned}
& 2^{-k-\beta} \sum_{i=1}^{k} \sum_{j=1}^{i} 2^{i+1-j} f\left(2^{\beta+j}\right)=2^{-k-\beta} \sum_{j=1}^{k} \sum_{i=j}^{k} f\left(2^{\beta+j}\right) 2^{i+1-j} \\
\leqslant & \sum_{j=1}^{k} f\left(2^{\beta+j}\right) 2^{2-j-\beta}=4 S_{\beta+1},
\end{aligned}
$$


on obtient $\frac{b_{n p+r}}{n p+r} \leqslant \frac{p b_{n}}{n p+r}+\frac{b_{r}}{n p+r}+4 S_{\beta+1}+2^{-k-\beta+1} \sum_{i=0}^{k+\beta+3} f\left(2^{i}\right)$. D'après le lemme 1.3.4, on a

$$
\limsup _{m \rightarrow+\infty} \frac{b_{m}}{m} \leqslant \liminf _{n \rightarrow+\infty}\left(\frac{b_{n}}{n}+4 S_{\left\lfloor\log _{2} n\right\rfloor+1}\right)=\liminf _{n \rightarrow+\infty} \frac{b_{n}}{n} .
$$

Donc la suite $\left(b_{n} / n\right)_{n \geqslant 1}$ est convergente.

Pour le cas général, en appliquant le résultat obtenu à la sous-suite $\left(b_{n_{0} k}\right)_{k \geqslant 1}$ et à la fonction $g(k)=f\left(n_{0} k\right)$, on obtient que la suite $\left(b_{n_{0} k} / k\right)_{k \geqslant 1}$ a une limite dans $\left[0,+\infty\left[\right.\right.$. D'autre part, si $l$ est un entier tel que $n_{0} \leqslant l<2 n_{0}$, alors, pour tout entier $k \geqslant 1$, on a l'encadrement

(12) $b_{n_{0}(k+3)}-b_{3 n_{0}-l}-f\left(n_{0} k+l\right)-f\left(3 n_{0}-l\right) \leqslant b_{n_{0} k+l} \leqslant b_{n_{0} k}+b_{l}+f\left(n_{0} k\right)+f(l)$.

En divisant (12) par $n_{0} k+l$, par passage à la limite quand $k \rightarrow+\infty$, on obtient

$$
\lim _{k \rightarrow+\infty} \frac{b_{n_{0} k+l}}{n_{0} k+l}=\lim _{k \rightarrow+\infty} \frac{b_{n_{0} k}}{n_{0} k}
$$

Comme $l$ est arbitraire, la démonstration est achevée.

Corollaire 1.3.6. - Soient $\left(a_{n}\right)_{n \geqslant 1}$ une suite de nombres réels, $f: \mathbb{Z}_{>0} \rightarrow \mathbb{R}$ une application croissante et $c>0$ une constante. On suppose que

1) pour tous entiers $n$ et $m$ assez grands, on ait $a_{n+m} \geqslant a_{n}+a_{m}-f(n)-f(m)$,

2) $a_{n} \leqslant$ cn pour tout entier $n \geqslant 1$,

3) $\sum_{\alpha \geqslant 0} f\left(2^{\alpha}\right) / 2^{\alpha}<+\infty$.

Alors la suite $\left(a_{n} / n\right)_{n \geqslant 1}$ admet une limite dans $\mathbb{R}$.

Démonstration. - On considère la suite $\left(b_{n}\right)_{n \geqslant 1}$ telle que $b_{n}=c n-a_{n}$. C'est une suite de nombres réels positifs. Si $n$ et $m$ sont deux entiers assez grands, on a

$b_{n+m}=c(n+m)-a_{n+m} \leqslant c n+c m-a_{n}-a_{m}+f(n)+f(m)=b_{n}+b_{m}+f(n)+f(m)$.

D'après la proposition 1.3.5, la suite $\left(b_{n} / n\right)_{n \geqslant 1}$ admet une limite dans $\mathbb{R}$. Comme $a_{n} / n=c-b_{n} / n$, la suite $\left(a_{n} / n\right)_{n \geqslant 1}$ admet aussi une limite dans $\mathbb{R}$.

\subsection{Quelques faits dans la géométrie algébrique}

Soit $S$ un schéma. On appelle fibré vectoriel sur $S$ tout $\theta_{S}$-module localement libre de rang fini. Un fibré vectoriel de rang 1 sur $S$ s'appelle aussi un fibré inversible sur $S$. Si $\mathcal{E}$ est un fibré vectoriel sur $S$, on utilise le symbole $\mathbb{P}(\mathcal{E})$ pour désigner le foncteur

$$
\begin{array}{clc}
\text { Schema } / S & \longrightarrow & \text { Ensemble } \\
(p: X \rightarrow S) & \longmapsto & \left\{\begin{array}{c}
\text { quotient localement } \\
\text { libre de rang } 1 \text { de } p^{*} \mathcal{E}
\end{array}\right\}
\end{array}
$$


On désigne par $\Theta_{\mathcal{E}}(1)$ le faisceau inversible sur $\mathbb{P}(\mathcal{E})$ l'objet universelle du foncteur représentable (13). Pour tout entier $m \geqslant 1$, l'expression $\theta_{\mathcal{E}}(m)$ désigne la puissance tensorielle $\theta_{\mathscr{E}}(1)^{\otimes m}$, et $\theta_{E}(-m)$ désigne $\theta_{E}(m)^{\vee}$.

Soit $X$ un schéma quasi-compact et quasi-séparé. On dit qu'un $\theta_{X}$-module inversible $L$ est ample (cf. [25] II.4.5.5 et [27] IV.1.7.14) si, pour tout $\Theta_{X}$-module quasi-cohérent et de type fini $\mathcal{F}$, il existe un entier $n_{0}>0$ tel que, pour tout entier $n \geqslant n_{0}, \mathcal{F} \otimes L^{\otimes n}$ soit engendré par ses sections globales (autrement dit, il existe un entier $a>0$ ainsi qu'un homomorphisme surjectif de $\theta_{X}^{\oplus a}$ vers $\left.\mathcal{F} \otimes L^{\otimes n}\right)$.

Soit $f$ un morphisme quasi-compact d'un schéma quasi-compact et quasi-séparé $X$ vers un schéma $Y$. On dit qu'un $\Theta_{X}$-module inversible $L$ est ample relativement à $f$ (cf. [25] II.4.6.1) s'il existe un recouvrement $\left(U_{\alpha}\right)$ de $Y$ par des ouverts affines tel que $\left.L\right|_{U_{\alpha}}$ soit ample sur $f^{-1}\left(U_{\alpha}\right)$ pour tout $\alpha$. Cela revient à dire que, pour tout $\vartheta_{X}$-module quasi-cohérent et de type fini $\mathcal{F}$, il existe un entier $n_{0}>0$ tel que, pour tout entier $n \geqslant n_{0}$, l'homomorphisme canonique $f^{*}\left(f_{*}\left(\mathcal{F} \otimes L^{\otimes n}\right)\right) \longrightarrow \mathcal{F} \otimes L^{\otimes n}$ soit surjectif (cf. [25] II.4.6.8 et [27] IV.1.7.15). Si $Y$ est affine, cette condition équivaut à l'amplitude de $L$ sur $X$.

Dans [29] (voir aussi [30]) Hartshorne a étendu la notion d'amplitude aux faisceaux localement libre de rang fini. Soit $X$ un schéma quasi-compact et quasi-séparé. On dit qu'un $\theta_{X}$-module localement libre de rang fini $E$ est ample si, pour tout $\Theta_{X}$-module quasi-cohérent de type fini $\mathcal{F}$, il existe un entier $n_{0}>0$ tel que, pour tout entier $n \geqslant n_{0}$, le faisceau $S^{n} E \otimes \mathcal{F}$ soit engendré par ses sections au-dessus de $X$, c'est-àdire que $S^{n} E \otimes \mathcal{F}$ est un quotient d'un $\vartheta_{X}$-module libre de rang fini, où $S^{n} E$ désigne la $n^{\text {ième }}$ puissance symétrique de $E$.

Soit $f: X \rightarrow Y$ un morphisme quasi-compact d'un schéma quasi-compact et quasiséparé vers un schéma. On dit qu'un $\Theta_{X}$-module localement libre de rang fini $E$ est ample relativement à $f$ (ou à $Y$ ) s'il existe un recouvrement de $Y$ par des ouverts affines $\left(U_{\alpha}\right)$ de telle sorte que $\left.E\right|_{U_{\alpha}}$ soit ample sur $f^{-1}\left(U_{\alpha}\right)$ pour tout $\alpha$. Lorsque $Y$ est localement noethérien et $f$ est de type fini, l'amplitude de $E$ relativement à $Y$ est équivalente à l'amplitude de $\theta_{E}(1)$ relativement à $Y$.

On rappelle deux résultats classiques concernant les fibrés inversibles amples que l'on utilisera plus loin dans le chapitre 4.

Proposition 1.4.1. - Soit $\pi: X \rightarrow Y$ un morphisme surjectif de schémas projectifs définis sur un corps $k$. Si $L$ est un $\vartheta_{X}$-module inversible ample relativement $\grave{a} \pi$, alors il existe un $\vartheta_{Y}$-module inversible ample $M$ tel que $L \otimes \pi^{*} M$ soit ample.

Démonstration. - Comme $L$ est ample relativement à $\pi$, il existe un entier $n>0$, un $\vartheta_{Y}$-module localement libre de rang fini et non-nul $E$ et une immersion $f: X \rightarrow \mathbb{P}(E)$ compatible à $\pi$ tels que $L^{\otimes n} \cong f^{*}\left(\Theta_{E}(1)\right)$. On désigne par $p: \mathbb{P}(E) \rightarrow X$ la projection 
canonique et considère le diagramme commutatif suivant :

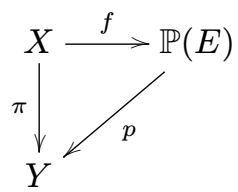

Comme $\pi$ est propre, le morphisme $f$ est une immersion fermée. Comme $Y$ est projectif, il existe un $\theta_{Y}$-module inversible ample $M$ tel que $E \otimes M^{\otimes n}$ soit encore ample. On a alors $\mathbb{P}(E) \cong \mathbb{P}\left(E \otimes M^{\otimes n}\right)$, et $\vartheta_{E \otimes M \otimes n}(1) \cong \theta_{E}(1) \otimes p^{*} M^{\otimes n}$. Comme $f$ est une immersion fermée,

$$
\left(L \otimes \pi^{*} M\right)^{\otimes n}=f^{*}\left(\theta_{E}(1)\right) \otimes f^{*}\left(p^{*} M\right)^{\otimes n}=f^{*}\left(\theta_{E}(1) \otimes p^{*} M^{\otimes n}\right) \cong f^{*}\left(\theta_{E \otimes M \otimes n}(1)\right)
$$

est ample puisque $E \otimes M^{\otimes n}$ est ample.

Proposition 1.4.2. - Soient $X$ un schéma intègre et noethérien, $\mathscr{L}$ un $\vartheta_{X}$-module inversible ample et $\mathcal{F}$ un $\vartheta_{X}$-module cohérent sans torsion. Alors il existe deux entiers $a, m>0$ ainsi qu'un homomorphisme injectif de $\mathcal{F}$ dans $\left(\mathscr{L}^{\otimes m}\right)^{\oplus a}$.

Démonstration. - Comme $\mathcal{F}$ est sans torsion, l'homomorphisme canonique

$$
\theta_{\mathscr{F}}: \mathcal{F} \longrightarrow \mathcal{F}^{\vee \vee}
$$

est injectif. Comme $\mathscr{L}$ est ample, il existe un entier $m>0$ tel que $\mathscr{L}^{\otimes m} \otimes \mathcal{F}^{\vee}$ soit engendré par ses sections au-dessus de $X$, i.e., il existe un entier $a$ et un homomorphisme surjectif $\varphi: \theta_{X}^{\oplus a} \rightarrow \mathscr{L}^{\otimes m} \otimes \mathcal{F}^{\vee}$. Par dualité on obtient un homomorphisme injectif

$$
\mathscr{L}^{\vee \otimes m} \otimes \mathcal{F} \stackrel{1 \otimes \theta_{\mathscr{G}}}{\longrightarrow} \mathscr{L}^{\vee \otimes m} \otimes \mathcal{F}^{\vee \vee} \stackrel{\varphi^{\vee}}{\longrightarrow} \theta_{X}^{\oplus a}
$$

qui induit un homomorphisme injectif $\mathcal{F} \rightarrow\left(\mathscr{L}^{\otimes m}\right)^{\oplus a}$.

Soit $k$ un corps. On entend variété projective sur Spec $k$ tout schéma intègre et projectif sur Spec $k$. Si $X$ est une variété projective de dimension $d$ sur Spec $k$ et si $L$ est un $\theta_{X}$-module inversible, le volume (cf. [33, 2.2.31]) de $L$ est par définition le nombre réel

$$
\operatorname{vol}(L):=\limsup _{n \rightarrow+\infty} \frac{\operatorname{rg}_{k} H^{0}\left(X, L^{\otimes n}\right)}{n^{d} / d !},
$$

où "limsup" peut être remplacé par "lim", grâce au théorème d'approximation de Fujita (cf. $[34,11.4 .7])$. Le $\vartheta_{X}$-module inversible $L$ est dit gros si $\operatorname{vol}(L)>0$. Par définition, un $\vartheta_{X}$-module inversible est gros si et seulement si l'une de ses puissances tensorielles (positives) est gros. De plus, le produit tensoriel d'un fibré inversible gros avec un fibré inversible effectif (i.e. ayant une section globale non-nulle) est encore gros.

Si $L$ est ample, alors il est gros, et $\operatorname{vol}(L)=c_{1}(L)^{d}$. La réciproque n'est pas vraie en générale. Mais si $L$ est un $\vartheta_{X}$-module inversible gros, alors il existe un entier $m \geqslant 1$, 
un $\vartheta_{X}$-module inversible ample $A$, et un $\theta_{X}$-module inversible effectif $M$ tels que $L^{\otimes m}$ soit isomorphe à $A \otimes M$ (cf. [33, 2.2.7]).

Proposition 1.4.3. - Soient $X$ une variété projective sur $\operatorname{Spec} k$ et $L$ un $\vartheta_{X}$-module inversible gros.

1) Si $\mathscr{L}$ est un $\vartheta_{X}$-module inversible, alors il existe un entier $q \geqslant 1$ et un homomorphisme injectif de $\mathscr{L}$ vers $L^{\otimes q}$, et il existe un entier $n \geqslant 1$ tel que $\mathscr{L} \otimes L^{\otimes n}$ soit gros.

2) Si $\mathcal{F}$ est un $\bigoplus_{X}$-module cohérent sans torsion, alors il existe deux entiers strictement positifs a et $m$ ainsi qu'un homomorphisme injectif de $\mathcal{F}$ dans $\left(L^{\otimes m}\right)^{\oplus a}$.

Démonstration. - Quitte à remplacer $L$ par l'une de ses puissances tensorielles, on peut supposer que $L$ s'écrit sous la forme $A \otimes M$, où $A$ est un $\vartheta_{X}$-module inversible ample, $M$ est effectif.

1) D'après la définition de l'amplitude, on obtient qu'il existe deux entiers $q \geqslant 1$ et $n \geqslant 1$ tels que $\mathscr{L}^{\vee} \otimes A^{\otimes q}$ soit effectif et que $\mathscr{L} \otimes A^{\otimes n}$ soit ample. Comme $M$ est effectif, il en est de même de toutes ses puissances tensorielles. On en déduit donc $\mathscr{L}^{\vee} \otimes L^{\otimes q}$ est effectif, et que $\mathscr{L} \otimes L^{\otimes n}$ est gros.

2) Il suffit d'appliquer 1.4 .2 au $\Theta_{X}$-module inversible ample $A$ pour obtenir un homomorphisme injectif de $\mathcal{F}$ vers certain $\left(A^{\otimes m}\right)^{\oplus a}$ et puis utiliser le fait que $M$ est effectif à construire un homomorphisme injectif $\left(A^{\otimes m}\right)^{\oplus a} \rightarrow\left(L^{\otimes m}\right)^{\oplus a}$.

On appelle courbe projective sur Spec $k$ toute variété projective de dimension 1 sur Spec $k$. Soient $C$ une courbe projective régulière sur Spec $k$ et $\pi: X \rightarrow C$ un $k$-morphisme projectif et plat d'une variété projective $X$ (sur Spec $k$ ) vers $C$. Pour tout $\theta_{X}$-module inversible $L$ sur $X$, le $\theta_{C}$-module cohérent $\pi_{*}(L)$ est sans torsion puisque le morphisme $\pi$ est plat. Comme $C$ est régulière, on obtient que $\pi_{*}(L)$ est en fait un fibré vectoriel sur $C$.

LEMme 1.4.4. - Soit $\pi: X \rightarrow C$ un k-morphisme projectif et plat d'une variété projective $X$ vers une courbe projective régulière sur Spec $k$. Soit $K^{\prime}=k(C)$ le corps des fonctions rationnelles sur $C$. Si L est un $\theta_{X}$-module inversible tel que $L_{K^{\prime}}$ soit effectif (sur $X_{K^{\prime}}$ ), alors il existe un $\theta_{C}$-module inversible ample $M$ tel que $L \otimes \pi^{*} M$ soit effectif sur $X$.

Démonstration. - Comme $L_{K^{\prime}}$ est effectif, le fibré vectoriel $\pi_{*}(L)$ est non-nul. On prend un $\theta_{C}$-module inversible ample $M$ tel que $\pi_{*}(L) \otimes M$ admet une section globale non-nulle. L'espace $H^{0}\left(X, L \otimes \pi^{*} M\right)=H^{0}\left(C, \pi_{*}(L) \otimes M\right)$ est donc non-nul.

Proposition 1.4.5. - Avec les notations du lemme 1.4.4, si $L_{K^{\prime}}$ est gros, alors il existe un $\theta_{C}$-module inversible $M$ tel que $L \otimes \pi^{*} M$ soit gros. 
Démonstration. - Soit $\mathscr{L}$ un $\Theta_{X^{-}}$-module inversible ample. Comme $L_{K^{\prime}}$ est gros, il existe un entier $n \geqslant 1$ tel que $L_{K^{\prime}}^{\otimes n} \otimes \mathscr{L}_{K^{\prime}}^{\vee}$ soit effectif. D'après le lemme 1.4.4, il existe un $\theta_{C}$-module inversible effectif $M$ tel que $L^{\otimes n} \otimes \mathscr{L}^{\vee} \otimes \pi^{*} M$ soit effectif. Dans ce cas-là, le $\vartheta_{X}$-module $L^{\otimes n} \otimes \pi^{*} M^{\otimes n} \otimes \mathscr{L}^{\vee}$ est également effectif. Par conséquent, $\left(L \otimes \pi^{*} M\right)^{\otimes n}$ s'écrit comme le produit tensoriel d'un fibré inversible ample et d'un fibré inversible effectif. Donc $L \otimes \pi^{*} M$ est gros. 



\section{CHAPITRE 2}

\section{FILTRATIONS DE HARDER-NARASIMHAN}

Les notions de filtration de Harder-Narasimhan et de polygone de HarderNarasimhan d'un fibré vectoriel sur une courbe projective régulière sont introduites par Harder et Narasimhan [28]. Leurs avatars en géométrie d'Arakelov sont dévélopés par Stuhler [42] et Grayson [24] pour les fibrés vectoriels hermitiens. Récemment Gaudron [22] a généralisé ces notions dans un cadre plus général des fibrés vectoriels adéliques.

Classiquement la filtration de Harder-Narasimhan d'un fibré vectoriel $\mathcal{E}$ est un drapeau de $\mathcal{E}$ dont les sous-quotients sont semi-stables et de pentes strictement décroissantes, et le polygone de Harder-Narasimhan de $\mathcal{E}$ est une fonction concave et linéaire par morceaux définie sur $[0, \mathrm{rg} E]$ dont les pentes successives sont les pentes des sous-quotients dans la filtration de Harder-Narasimhan. En géométrie d'Arakelov, le polygone de Harder-Narasimhan est un invariant arithmétique très général qui permet de considérer toutes les pentes d'un fibré vectoriel hermitien (ou adélique) en même temps (cf. [6] et [22]).

Dans ce chapitre, on introduit un nouveau point de vue de cette théorie. À chaque fibré adélique hermitien, on associe une $\mathbb{R}$-filtration de l'espace vectoriel sous-jacent, qui mémorise en même temps le drapeau et les pentes successives. On vérifie que le polygone associé à cette $\mathbb{R}$-filtration coïncide avec une forme normalisée du polygone de Harder-Narasimhan du fibré adélique hermitien. Ce résultat nous permet d'utiliser la méthode des $\mathbb{R}$-filtrations et des mesures à étudier les fibrés adéliques hermitiens.

On rappelle que le symbole $K$ désigne un corps de nombres, comme convenu dans le sous-paragraphe 1.1 .

\subsection{Fibrés vectoriels adéliques}

Les fibrés vectoriels adéliques, notamment les fibrés vectoriels hermitiens sont des objets fondamentaux de la théorie des pentes. Depuis l'article fondateur [6] de J.B. Bost, les fibrés vectoriels adéliques ainsi que leurs invariants arithmétiques ont été systématiquement étudiés dans une série d'articles tels que $[\mathbf{7}, \mathbf{8 , 1 1 , ~ 1 5 , ~ 2 2 ] . ~}$ Dans cette section, on rappelle des notions basiques de cette théorie et des résultats 
classiques que l'on utilisera plus loin. Voir [22] pour une présentation complète et détaillée.

\subsubsection{Définition, degré d'Arakelov et pente}

DÉFInition 2.1.1. - On appelle fibré vectoriel adélique sur Spec $K$ toute donnée $\bar{E}=$ $\left(E,\left(\|\cdot\|_{v}\right)_{v \in \Sigma_{f} \cup \Sigma_{\infty}}\right)$ d'un espace vectoriel $E$ de rang fini sur $K$ et d'une famille de normes $\|\cdot\|_{v}$ sur $E \otimes_{K} \mathbb{C}_{v}$, soumise aux conditions suivantes :

1) Il existe une base $\left(s_{1}, \ldots, s_{r}\right)$ de $E$ sur $K$ et une partie finie $S$ de $\Sigma_{f}$ telles que, $\left\|a_{1} s_{1}+\cdots+a_{r} s_{r}\right\|_{v}=\max \left(\left|a_{1}\right|_{v}, \ldots,\left|a_{r}\right|_{v}\right)$ quels que soient $v \in \Sigma_{f} \backslash S$ et $\left(a_{1}, \ldots, a_{r}\right) \in \mathbb{C}_{v}^{r}$.

2) Pour toute place $v \in \Sigma_{f} \cup \Sigma_{\infty}$, la norme $\|\cdot\|_{v}$ est invariante sous l'action du groupe $\operatorname{Gal}\left(\mathbb{C}_{v} / K_{v}\right)$. Plus précisément, si $\left(s_{1}, \ldots, s_{r}\right)$ est une base de $E_{K_{v}}$ sur $K_{v}$ et si $a_{1}, \ldots, a_{r}$ sont des éléments dans $\mathbb{C}_{v}$, alors

$$
\left\|\tau\left(a_{1}\right) s_{1}+\cdots+\tau\left(a_{r}\right) s_{r}\right\|_{v}=\left\|a_{1} s_{1}+\cdots+a_{r} s_{r}\right\|_{v}
$$

quel que soit $\tau \in \operatorname{Gal}\left(\mathbb{C}_{v} / K_{v}\right)$.

3) Si $\mathfrak{p} \in \Sigma_{f}$, alors la norme $\|\cdot\|_{\mathfrak{p}}$ est ultramétrique, c'est-à-dire $\left\|s+s^{\prime}\right\|_{\mathfrak{p}} \leqslant$ $\max \left(\|s\|_{\mathfrak{p}},\left\|s^{\prime}\right\|_{\mathfrak{p}}\right)$

Le fibré vectoriel adélique $\bar{E}$ est dit $n u l$ si son espace vectoriel sous-jacent $E$ est nul. En outre, le rang de $\bar{E}$ est par définition le rang de $E$ sur $K$. Un fibré vectoriel adélique de rang 1 s'appelle aussi un fibré inversible adélique.

Remarque 2.1.2. - Certains auteurs ont considéré des fibrés vectoriels normés (ou semi-normés) plus généraux. Par exemple, dans [41, Definition 1.A], au lieu de s'imposer la condition 1) comme ci-dessus, les auteurs ont proposé une condition plus faible:

pour tout $s \in E$, il existe un sous-ensemble fini $S$ de $\Sigma_{f}$ tel que $\|s\|_{v} \leqslant 1$ quelle que soit $v \in \Sigma_{f} \backslash S$.

Il devrait être intéressant de savoir dans quelle mesure la théorie de pentes peut être généralisée dans ce cadre.

Soit $\bar{E}$ un fibré vectoriel adélique non-nul sur $\operatorname{Spec} k$. On définit la boule unité de $\bar{E}$ comme le sous-ensemble $\mathbb{B}(\bar{E})$ de $E \otimes_{K} \mathbf{A}_{K}$ des éléments $\left(s_{v}\right)_{v \in \Sigma_{f} \cup \Sigma_{\infty}}$ tel que $\left\|s_{v}\right\|_{v} \leqslant 1$ quelle que soit $v \in \Sigma_{f} \cup \Sigma_{\infty}$. La caractéristique d'Euler-Poincaré de $\bar{E}$ est par définition le nombre réel

$$
\chi(\bar{E}):=\log (\operatorname{vol}(\mathbb{B}(\bar{E})))-\log (\operatorname{covol}(E)),
$$

où vol est une mesure de Haar quelconque sur $E \otimes_{K} \mathbf{A}_{\mathbf{K}}$, et $\operatorname{covol}(E)$ est le covolume de $E$ pour cette mesure, c'est-à-dire la mesure pour vol d'un domaine fondamental du quotient $\left(E \otimes_{K} \mathbf{A}_{\mathbf{K}}\right) / E$. Cette définition ne dépend pas du choix de la mesure de Haar vol. En particulier, on a l'égalité

$$
\chi(\bar{E})=\log \left(\mathbf{m}_{E}(\mathbb{B}(\bar{E}))\right)-\operatorname{rg}(E) \log \sqrt{\left|D_{K}\right|},
$$

où $\mathbf{m}_{E}$ est la mesure de Haar définie dans $\S 1.1$. 
Pour tout entier $r \geqslant 1$, soit $\bar{K}^{r}=\left(K^{r},\left(\|\cdot\|_{v}\right)_{v \in \Sigma_{f} \cup \Sigma_{\infty}}\right)$ l'espace vectoriel de rang $r$ sur $K$ muni des métriques qui "proviennent de celles de $K$ ". Plus précisément, si on désigne par $\left(e_{1}, \ldots, e_{r}\right)$ la base canonique de $K^{r}$, alors pour toute place $v$ de $K$ et tous éléments $a_{1}, \ldots, a_{r}$ dans $\mathbb{C}_{v}$, on a

$$
\left\|a_{1} e_{1}+\cdots+a_{r} e_{r}\right\|_{v}= \begin{cases}\max \left(\left|a_{1}\right|_{v}, \ldots,\left|a_{r}\right|_{v}\right), & \text { si } v \in \Sigma_{f}, \\ \sqrt{\left|a_{1}\right|_{v}^{2}+\cdots+\left|a_{r}\right|_{v}^{2}}, & \text { si } v \in \Sigma_{\infty} .\end{cases}
$$

$\bar{K}^{r}$ est donc un fibré vectoriel adélique $\operatorname{sur} \operatorname{Spec} K$, appelé le fibré vectoriel adélique trivial de rang $r$ sur $\operatorname{Spec} K$.

Si $\bar{E}$ est un fibré vectoriel adélique de $\operatorname{rang} r>0$ sur $\operatorname{Spec} K$, on définit le degré d'Arakelov de $\bar{E}$ comme la différence

$$
\widehat{\operatorname{deg}}(\bar{E}):=\chi(\bar{E})-\chi\left(\bar{K}^{r}\right)=\log (\operatorname{vol}(\mathbb{B}(\bar{E})))-\log \left(\operatorname{vol}\left(\mathbb{B}\left(\bar{K}^{r}\right)\right)\right) .
$$

Sa version normalisée est par définition $\widehat{\operatorname{deg}}_{n}(\bar{E})=\frac{1}{[K: \mathbb{Q}]} \widehat{\operatorname{deg}}(\bar{E})$. La pente de $\bar{E}$ est définie comme le quotient $\widehat{\mu}(\bar{E}):=\widehat{\operatorname{deg}}_{n}(\bar{E}) / \operatorname{rg} E$. En particulier, le degré d'Arakelov d'un fibré vectoriel adélique trivial sur $\operatorname{Spec} K$ est nul. Enfin, si $\bar{E}$ est nul, alors son degré d'Arakelov et sa pente sont par convention nuls.

Proposition 2.1.3. - La caractéristique d'Euler-Poincaré de $\bar{K}^{n}$ est

$$
\chi\left(\bar{K}^{n}\right)=\left(\# \Sigma_{\infty, r}\right) \log V_{n}+\left(\# \Sigma_{\infty, c}\right)\left(\log V_{2 n}+n \log 2\right)-n \log \sqrt{\left|D_{K}\right|},
$$

où $V_{n}$ est le volume euclidien standard du disque unité dans $\mathbb{R}^{n}$, $D_{K}$ est le discriminant de $K$.

Démonstration. - C'est une conséquence de la formule (15) ainsi que la définition de $\mathbf{m}_{E}$.

Remarque 2.1.4. - D'après [22] (10), on a

$$
\log V_{n}=n\left(\log V_{1}+\log \Gamma(3 / 2)\right)-\log \Gamma(1+n / 2)=-\frac{n}{2} \log n+O(n) .
$$

On en déduit donc

$$
\chi\left(\bar{K}^{n}\right)=-\frac{[K: \mathbb{Q}]}{2} n \log n+O(n)
$$

puisque $\# \Sigma_{\infty, r}+2 \# \Sigma_{\infty, c}=[K: \mathbb{Q}]$.

Si $\bar{L}$ est un fibré inversible adélique et si $s$ est un élément non-nul de $L$, alors le degré d'Arakelov de $\bar{L}$ se calcule comme ci-dessous :

$$
\widehat{\operatorname{deg}}(\bar{L})=-\sum_{v \in \Sigma_{f} \cup \Sigma_{\infty}} n_{v} \log \|s\|_{v} .
$$

Si $\bar{L}_{1}$ et $\bar{L}_{2}$ sont deux fibrés inversibles adéliques, alors leur produit tensoriel $\bar{L}_{1} \otimes \bar{L}_{2}$ est par définition le fibré inversible adélique dont l'espace vectoriel sous-jacent est 
$L_{1} \otimes L_{2}$ et dont les métriques satisfont $\left\|s_{1} \otimes s_{2}\right\|_{v}=\left\|s_{1}\right\|_{v}\left\|s_{2}\right\|_{v}$ quelle que soit $v$. La formule (18) implique que

$$
\widehat{\operatorname{deg}}\left(\bar{L}_{1} \otimes \bar{L}_{2}\right)=\widehat{\operatorname{deg}}\left(\bar{L}_{1}\right)+\widehat{\operatorname{deg}}\left(\bar{L}_{2}\right)
$$

Soient $\bar{E}$ et $\bar{F}$ deux fibrés vectoriels adéliques sur Spec $K$. Si $\varphi: E \rightarrow F$ est une application $K$-linéaire, alors elle induit pour chaque place $v \in \Sigma_{f} \cup \Sigma_{\infty}$ une application $\mathbb{C}_{v}$-linéaire $\varphi_{v}: E \otimes_{K} \mathbb{C}_{v} \rightarrow F \otimes_{K} \mathbb{C}_{v}$ par extension de scalaire. On note $h_{v}(\varphi)=\log \left\|\varphi_{v}\right\|_{v}$ et on l'appelle la hauteur locale de $\varphi$ en $v$, où $\left\|\varphi_{v}\right\|_{v}$ est la norme d'opérateur linéaire. On définit la hauteur de $\varphi$ comme la somme normalisée

$$
h(\varphi):=\frac{1}{[K: \mathbb{Q}]} \sum_{v \in \Sigma_{f} \cup \Sigma_{\infty}} n_{v} \log \left\|\varphi_{v}\right\|_{v} .
$$

2.1.2. Inégalité de pentes. - La proposition suivante est une inégalité de pentes. Voir [22] Lemmes 6.2 et 6.3 pour une preuve. Cette inégalité nous permet de comparer les pentes lorsque l'on change les métriques d'un fibré vectoriel adélique.

Proposition 2.1.5. - Soient $\bar{E}$ et $\bar{F}$ deux fibrés vectoriels adéliques de rang $r>0$ sur $\operatorname{Spec} K$. Si $\varphi: E \rightarrow F$ est un isomorphisme K-linéaire, alors

$$
\widehat{\mu}(\bar{E}) \leqslant \widehat{\mu}(\bar{F})+h(\varphi) .
$$

Si de plus $r=1$, alors l'inégalité (21) est en fait une égalité.

La pente maximale de $\bar{E}$ est par définition la valeur maximale des pentes des sousfibrés vectoriels adéliques (c'est-à-dire sous-espace de $E$ muni des métriques induites) non-nuls de $\bar{E}$, notée $\widehat{\mu}_{\max }(\bar{E})$. Par convention $\widehat{\mu}_{\max }(0)=-\infty$. L'existence de la pente maximale est justifiée dans [22, proposition 5.3]. On rappelle ici une inégalité dans le lemme 6.4 loc. cit. qui compare les pentes maximales de la source et du but d'un homomorphisme injectif.

Proposition 2.1.6. - Soient $\bar{E}$ et $\bar{F}$ deux fibrés vectoriels adéliques et $\varphi: E \rightarrow F$ une application K-linéaire injective. Alors

$$
\widehat{\mu}_{\max }(\bar{E}) \leqslant \widehat{\mu}_{\max }(\bar{F})+h(\varphi) .
$$

De façon similaire, la pente mimimale d'un fibré vectoriel adélique non-nul $\bar{E}$ est par définition la valeur minimale des pentes des quotients (c'est-à-dire espace quotient de $E$ muni des métriques quotients) non-nuls de $\bar{E}$. La pente minimale du fibré nul est par convention $+\infty$. Par définition on a la relation $\widehat{\mu}_{\min }(\bar{E}) \leqslant \widehat{\mu}(\bar{E}) \leqslant \widehat{\mu}_{\max }(\bar{E})$ si $E$ est non-nul.

En utilisant la proposition 2.1.5, on obtient l'inégalité suivante qui compare les pentes minimales étant donné un homomorphisme surjectif entre les fibrés vectoriels adéliques. 
Proposition 2.1.7. - Soient $\bar{E}$ et $\bar{F}$ deux fibrés vectoriels adéliques, et $\varphi: E \rightarrow F$ une application K-linéaire surjective et non-nulle. Alors

$$
\widehat{\mu}_{\min }(\bar{E}) \leqslant \widehat{\mu}_{\min }(\bar{F})+h(\varphi) .
$$

Démonstration. - Soit $\bar{G}$ un quotient de $\bar{F}$ tel que $\widehat{\mu}(\bar{G})=\widehat{\mu}_{\min }(\bar{F})$. Soit $\bar{G}^{\prime}$ le fibré vectoriel adélique qui admet $G$ comme espace vectoriel sous-jacent et dont les métriques sont des métriques quotients de celles $\bar{E}$. La hauteur de l'application d'identité $\bar{G}^{\prime} \rightarrow \bar{G}$ est majorée par $h(\varphi)$. D'après la proposition 2.1.5, on a

$$
\widehat{\mu}_{\min }(\bar{E}) \leqslant \widehat{\mu}\left(\bar{G}^{\prime}\right) \leqslant \widehat{\mu}(\bar{G})+h(\varphi)=\widehat{\mu}_{\min }(\bar{F})+h(\varphi) .
$$

Corollaire 2.1.8. - Étant donnés deux fibrés vectoriels adéliques $\bar{E}$ et $\bar{F}$, et une application $K$-linéaire non-nulle $\varphi: E \rightarrow F$, on a

$$
\widehat{\mu}_{\min }(\bar{E}) \leqslant \widehat{\mu}_{\max }(\bar{F})+h(\varphi) .
$$

Démonstration. - On désigne par $\bar{G}$ l'image de $E$ par $\varphi$, munie des métriques induites de celles de $\bar{F}$. En appliquant (23) à l'homomorphisme canonique $\bar{E} \rightarrow \bar{G}$, on obtient

$$
\widehat{\mu}_{\min }(\bar{E}) \leqslant \widehat{\mu}_{\min }(\bar{G})+h(\varphi) \leqslant \widehat{\mu}(\bar{G})+h(\varphi) \leqslant \widehat{\mu}_{\max }(\bar{F})+h(\varphi),
$$

qui démontre (24).

2.1.3. Fibrés adéliques hermitiens. - On dit qu'un fibré vectoriel adélique $\bar{E}=$ $\left(E,\left(\|\cdot\|_{v}\right)_{v \in \Sigma_{f} \cup \Sigma_{\infty}}\right)$ sur Spec $K$ est un fibré adélique hermitien si, pour chaque place infinie $v \in \Sigma_{\infty}$, la norme $\|\cdot\|_{v}$ est induite par un produit hermitien $\langle,\rangle_{v}$ sur $E \otimes_{K} \mathbb{C}_{v}$. Par définition, les fibrés inversibles adéliques sont des fibrés adéliques hermitiens. Si $\bar{E}$ et $\bar{F}$ sont deux fibrés adéliques hermitiens, on appelle homomorphisme de $\bar{E}$ vers $\bar{F}$ toute application $K$-linéaire de $E$ vers $F$.

Remarque 2.1.9. - Dans la littérature, la notion de fibré vectoriel normé (surtout celle de fibré vectoriel hermitien) sur Spec $\theta_{K}$ est plus couramment utilisée. Un fibré vectoriel normé $\bar{\varepsilon}$ sur Spec $\theta_{K}$ est la donnée d'un $\theta_{K}$-module projectif $\delta$ et d'une famille de normes $\|\cdot\|_{v}$ sur $\mathscr{E} \otimes_{\vartheta_{K}} \mathbb{C}_{v}$, invariantes par la conjugaison complexe si la place $v$ est réelle. Une autre définition équivalente est la donnée $\left(\mathscr{E},\left(\|\cdot\|_{\sigma}\right)_{\sigma: K \rightarrow \mathbb{C}}\right)$ d'un $\theta_{K}$-module projectif $\mathcal{E}$ et d'une famille de métriques $\|\cdot\|_{\sigma}$ indexées par les plongements de $K$ dans $\mathbb{C}$, invariantes par la conjugaison complexe (on tient compte aussi de l'action de la conjugaison complexe sur les plongements).

Si pour toute place infinie $v$ (resp. pour tout plongement $\sigma: K \rightarrow \mathbb{C}$ ), la métrique $\|\cdot\|_{v}\left(\right.$ resp. $\left.\|\cdot\|_{\sigma}\right)$ est hermitienne, on $\operatorname{dit}$ que $\left(\mathcal{E},\left(\|\cdot\|_{v}\right)_{v \in \Sigma_{\infty}}\right)\left(\operatorname{resp} .\left(\mathcal{E},\left(\|\cdot\|_{\sigma}\right)_{\sigma: K \rightarrow \mathbb{C}}\right)\right)$ est un fibré vectoriel hermitien $\operatorname{sur} \operatorname{Spec} \theta_{K}$.

Comme montré par Gaudron [22, §3], les fibrés vectoriels normés (resp. fibrés vectoriels hermitiens) sur $\operatorname{Spec} \theta_{K}$ sont étroitement liés aux fibrés vectoriel adéliques (resp. fibrés adéliques hermitiens) sur $\operatorname{Spec} K$. Soient $\overline{\mathcal{E}}$ un fibré vectoriel normé sur 
Spec $\theta_{K}$ et $E=\varepsilon_{K}$. Pour chaque place finie $\mathfrak{p} \in \Sigma_{f}$, la structure de $\theta_{K}$-module sur $\mathcal{E}$ induit une norme $\|\cdot\|_{\mathfrak{p}}$ sur $E \otimes_{K} \mathbb{C}_{\mathfrak{p}}$ :

$$
\|s\|_{\mathfrak{p}}=\inf \left\{|a|_{\mathfrak{p}} \mid a \in \mathbb{C}_{\mathfrak{p}}, s \in a\left(\mathcal{E} \otimes_{\vartheta_{K}} \widehat{\vartheta}_{\mathfrak{p}}\right)\right\},
$$

où $\widehat{\vartheta}_{\mathfrak{p}}$ est l'anneau de valuation de $\mathbb{C}_{\mathfrak{p}}$. Ainsi $\left(E,\left(\|\cdot\|_{v}\right)_{v \in \Sigma_{f} \cup \Sigma_{\infty}}\right)$ est un fibré vectoriel adélique sur $\operatorname{Spec} K$, qui est hermitien si $\overline{\mathcal{E}}$ est hermitien.

Réciproquement, étant donné un fibré vectoriel adélique $\bar{E}$ sur $\operatorname{Spec} K$, on peut en déduire une structure entière sur $\theta_{K}$. En effet, l'ensemble

$$
\mathcal{E}=\left\{x \in E \mid \forall \mathfrak{p} \in \Sigma_{f},\|x\|_{\mathfrak{p}} \leqslant 1\right\}
$$

est un $\theta_{K}$-module projectif de type fini. Si les métriques aux places finies sont discrètes (c'est-à-dire que, pour tout $\mathfrak{p} \in \Sigma_{f}$, il existe une base de $E$ sur $K$ qui est orthonormée pour la norme $\|\cdot\|_{\mathfrak{p}}$ ), alors pour chaque $\mathfrak{p} \in \Sigma_{f}$, la norme sur $E \otimes_{K} \mathbb{C}_{\mathfrak{p}}$ induite par la structure entière de $\mathcal{E}$ coïncide avec $\|\cdot\|_{\mathfrak{p}}$.

Étant donné un fibré adélique hermitien $\bar{E}$, les sous-fibrés adéliques hermitiens et les quotients adéliques hermitiens sont naturellement définis. Un sous-fibré adélique hermitien est la donnée $\bar{F}$ d'un sous-espace vectoriel $F$ de $E$ muni des métriques induites. L'espace quotient $E / F$ et les métriques quotientes forment aussi un fibré adélique hermitien sur $\operatorname{Spec} K$, noté $\bar{E} / \bar{F}$. Le diagramme $0 \longrightarrow \bar{F} \longrightarrow \bar{E} \longrightarrow \bar{F} \longrightarrow 0$ est appelé une suite exacte courte de fibrés adéliques hermitiens.

Plusieurs constructions algébriques sont "canoniquement" définies pour les fibrés adéliques hermitiens, notamment la somme directe et le produit tensoriel de deux fibrés adéliques hermitiens, ainsi que le dual, les puissances symétriques et les puissances extérieures d'un fibré adélique hermitien. En particulier, le déterminant d'un fibré adélique hermitien $\bar{E}$ est par définition la puissance extérieure dét $\bar{E}:=\Lambda^{\mathrm{rg} E} \bar{E}$. De plus, on a $\widehat{\operatorname{deg}}(\bar{E})=\widehat{\operatorname{deg}}(\operatorname{dét} \bar{E})$. D'autre part, $\widehat{\operatorname{deg}}(\bar{E})+\widehat{\operatorname{deg}}\left(\bar{E}^{\vee}\right)=0$, où $\bar{E}^{\vee}$ est le dual de $\bar{E}$.

Si $0 \longrightarrow \bar{F} \longrightarrow \bar{E} \longrightarrow \bar{G} \longrightarrow 0$ est une suite exacte courte de fibrés adéliques hermitiens, alors dét $\bar{E}$ est canoniquement isomorphe à dét $\bar{F} \otimes \operatorname{dét} \bar{G}$, d'où

$$
\widehat{\operatorname{deg}}(\bar{E})=\widehat{\operatorname{deg}}(\bar{F})+\widehat{\operatorname{deg}}(\bar{G}) .
$$

Si $\bar{E}$ et $\bar{F}$ sont deux fibrés adéliques hermitiens, alors dét $(\bar{E} \otimes \bar{F})$ est isomorphe à $(\operatorname{dét} \bar{E})^{\otimes \operatorname{rg} F} \otimes(\operatorname{dét} \bar{F})^{\otimes \operatorname{rg} E}$. Par conséquent,

$$
\widehat{\operatorname{deg}}(\bar{E} \otimes \bar{F})=\operatorname{rg}(F) \widehat{\operatorname{deg}}(\bar{E})+\operatorname{rg}(E) \widehat{\operatorname{deg}}(\bar{F}) .
$$

Les égalités de degrés d'Arakelov (25) et (26) impliquent les égalités suivantes de pentes

$$
\begin{gathered}
\widehat{\mu}(\bar{E})=\frac{\operatorname{rg} F}{\operatorname{rg} E} \widehat{\mu}(\bar{F})+\frac{\operatorname{rg} G}{\operatorname{rg} E} \widehat{\mu}(\bar{G}), \\
\widehat{\mu}(\bar{E} \otimes \bar{F})=\widehat{\mu}(\bar{E})+\widehat{\mu}(\bar{F}),
\end{gathered}
$$

pourvu que tous les fibrés adéliques hermitiens sont non-nuls. 
La propriété de dualité suivante est valable pour les fibrés adéliques hermitiens.

Proposition 2.1.10. - Si E est un fibré adélique hermitien, alors

$$
\widehat{\mu}_{\min }(\bar{E})=-\widehat{\mu}_{\max }\left(\bar{E}^{\vee}\right)
$$

Remarque 2.1.11. - L'égalité (29) n'est plus vraie en générale pour les fibrés vectoriels adéliques. Voir [22, Lemme 5.6] pour une majoration explicite de

$$
\left|\widehat{\mu}_{\max }\left(\bar{E}^{\vee}\right)+\widehat{\mu}_{\min }(\bar{E})\right|
$$

lorsque $E$ non-nul.

2.1.4. Pente minimale du produit tensoriel. - On rappelle une estimation de la pente minimales du produit tensoriel d'un nombre fini de fibrés adéliques hermitiens établie dans [17].

Proposition 2.1.12. - Soit $\left(\bar{E}_{i}\right)_{1 \leqslant i \leqslant n}$ une famille finie de fibrés adéliques hermitiens non-nuls sur $\operatorname{Spec} K$. L'inégalité suivante est vérifiée :

$$
\widehat{\mu}_{\min }\left(\bar{E}_{1} \otimes \cdots \otimes \bar{E}_{n}\right) \geqslant \sum_{i=1}^{n}\left(\widehat{\mu}_{\min }\left(\bar{E}_{i}\right)-\log \left(\operatorname{rg} E_{i}\right)\right) .
$$

Remarque 2.1.13. - 1) Dans [17] theorem 1.1, l'auteur a montré que

$$
\widehat{\mu}_{\max }\left(\bar{E}_{1} \otimes \cdots \otimes \bar{E}_{n}\right) \leqslant \sum_{i=1}^{n}\left(\widehat{\mu}_{\max }\left(\bar{E}_{i}\right)+\log \left(\operatorname{rg} E_{i}\right)\right) .
$$

Si on applique ce résultat à la famille $\left(\bar{E}_{i}^{\vee}\right)_{1 \leqslant i \leqslant n}$, en s'appuyant sur l'égalité $\widehat{\mu}_{\max }\left(\bar{E}^{\vee}\right)=-\widehat{\mu}_{\min }(\bar{E})$, on obtient $(30)$.

2) Dans [17], les estimations sont obtenues dans le cadre des fibrés vectoriels hermitiens sur Spec $\theta_{K}$, mais ces estimations restent valables avec le même terme d'erreur pour les fibrés adéliques hermitiens.

\subsubsection{Comparaison des fibrés vectoriels adéliques aux fibrés adéliques} hermitiens. - Soit $V$ un espace vectoriel de rang fini et non-nul sur $\mathbb{C}$. Soit $r=$ $\operatorname{rg}(V)$. Les normes sur $V$ se comparent aux normes hermitiennes via l'ellipsoïde de John et l' ellipsoïde de Löwner (cf. [22] définition-théorème 2.4, voir aussi [43] page 84). Plus précisément, si $\|\cdot\|$ est une norme sur $V$, alors il existe deux normes hermitiennes $\|\cdot\|_{\text {John }}$ et $\|\cdot\|_{\text {Löwner }}$ telles que

$$
\frac{1}{\sqrt{r}}\|x\|_{\text {John }} \leqslant\|x\| \leqslant\|x\|_{\text {John }}, \quad \text { et } \quad\|x\|_{\text {Löwner }} \leqslant\|x\| \leqslant \sqrt{r}\|x\|_{\text {Löwner }}
$$

quel que soit $x \in V$.

Si $\bar{E}=\left(E,\left(\|\cdot\|_{v}\right)_{v \in \Sigma}\right)$ est un fibré vectoriel adélique, on désigne par $\bar{E}_{\mathrm{J}}$ le fibré adélique hermitien $\left(E,\left(\|\cdot\|_{v}^{\prime}\right)_{v \in \Sigma}\right)$ qui admet $E$ comme espace vectoriel sous-jacent, et tel que

$$
\forall \mathfrak{p} \in \Sigma_{f},\|\cdot\|_{\mathfrak{p}}^{\prime}=\|\cdot\|_{\mathfrak{p}}, \quad \text { et } \quad \forall v \in \Sigma_{\infty},\|\cdot\|_{v}^{\prime}=\|\cdot\|_{v, \mathrm{John}} \text {. }
$$


De façon similaire, on désigne par $\bar{E}_{\mathrm{L}}$ le fibré adélique hermitien $\left(E,\left(\|\cdot\|_{v}^{\prime \prime}\right)_{v \in \Sigma}\right)$ qui admet $E$ comme espace vectoriel sous-jacent, et tel que

$$
\forall \mathfrak{p} \in \Sigma_{f},\|\cdot\|_{\mathfrak{p}}^{\prime}=\|\cdot\|_{\mathfrak{p}}, \quad \text { et } \quad \forall v \in \Sigma_{\infty},\|\cdot\|_{v}^{\prime}=\|\cdot\|_{v, \text { Löwner }}
$$

On en déduit les comparaisons suivantes :

Proposition 2.1.14. - Soit $\bar{E}$ un fibré vectoriel adélique de rang $r>0$ sur $\operatorname{Spec} K$. Alors on a les inégalités suivantes :

$$
\begin{gathered}
\widehat{\mu}(\bar{E})-\frac{1}{2} \log r \leqslant \widehat{\mu}\left(\bar{E}_{\mathrm{J}}\right) \leqslant \widehat{\mu}(\bar{E}) \leqslant \widehat{\mu}\left(\bar{E}_{\mathrm{L}}\right) \leqslant \widehat{\mu}(\bar{E})+\frac{1}{2} \log r \\
\widehat{\mu}_{\max }(\bar{E})-\frac{1}{2} \log r \leqslant \widehat{\mu}_{\max }\left(\bar{E}_{\mathrm{J}}\right) \leqslant \widehat{\mu}_{\max }(\bar{E}) \leqslant \widehat{\mu}_{\max }\left(\bar{E}_{\mathrm{L}}\right) \leqslant \widehat{\mu}_{\max }(\bar{E})+\frac{1}{2} \log r, \\
\widehat{\mu}_{\min }(\bar{E})-\frac{1}{2} \log r \leqslant \widehat{\mu}_{\min }\left(\bar{E}_{\mathrm{J}}\right) \leqslant \widehat{\mu}_{\min }(\bar{E}) \leqslant \widehat{\mu}_{\min }\left(\bar{E}_{\mathrm{L}}\right) \leqslant \widehat{\mu}_{\min }(\bar{E})+\frac{1}{2} \log r
\end{gathered}
$$

Démonstration. - Ce sont respectivement des conséquences des inégalités de pentes (21), (22) et (23).

2.1.6. Application à l'étude du produit tensoriel. - Soit $\left(\bar{E}_{i}\right)_{i=1}^{n}$ une famille finie de fibrés vectoriels adéliques. À l'espace tensoriel $E_{1} \otimes \cdots \otimes E_{n}$ il y a plusieurs façons "naturelles" de conférer une structure de fibré vectoriel adélique tensoriel. Ces constructions sont discutées en détail dans [22, §3.3]. On rappelle quelques constructions et résultats dans [22] que l'on utilisera plus loin dans le chapitre 4 .

Soit $n \geqslant 1$ un entier. On appelle norme tensorielle d'ordre $n$ la donnée $\alpha$ pour toute famille $\left(V_{i},\|\cdot\|\right)_{i=1}^{n}$ de $n$ espaces de Banach de dimension finie sur $\mathbb{C}$ d'une norme $\|\cdot\|_{\alpha}$ sur le produit tensoriel $V_{1} \otimes \cdots \otimes V_{n}$ telle que

(i) pour tout $\left(x_{1}, \ldots, x_{n}\right) \in V_{1} \times \cdots \times V_{n}$, on a

$$
\left\|x_{1} \otimes \cdots \otimes x_{n}\right\|_{\alpha} \leqslant \prod_{i=1}^{n}\left\|x_{i}\right\| ;
$$

(ii) si pour tout $i \in\{1, \ldots, n\}, \varphi_{i}: V_{i} \rightarrow W_{i}$ est une application linéaire d'espaces de Banach de dimension finie, alors la norme de l'application

$$
\varphi_{1} \otimes \cdots \otimes \varphi_{n}: V_{1} \otimes \cdots \otimes V_{n} \longrightarrow W_{1} \otimes \cdots \otimes W_{n}
$$

pour les normes $\|\cdot\|_{\alpha}$ est majorée par le produit des normes $\prod_{i=1}^{n}\left\|\varphi_{i}\right\|$.

On désigne par $V_{1} \otimes_{\alpha} \cdots \otimes_{\alpha} V_{n}$ l'espace tensoriel $V_{1} \otimes \cdots \otimes V_{n}$ muni de la norme $\|\cdot\|_{\alpha}$. On dit que $\alpha$ est une norme tensorielle hermitienne si, pour toute famille $\left(V_{i}\right)_{i=1}^{n}$ d'espaces hermitiens, l'espace normé $V_{1} \otimes_{\alpha} \cdots \otimes_{\alpha} V_{n}$ s'identifie au produit tensoriel hermitien usuel de $V_{1}, \ldots, V_{n}$. Autrement dit, si pour tout $i \in\{1, \ldots, n\}$, $\mathbf{e}^{(i)}$ est une base orthonormée de $V_{i}$, alors

$$
\mathbf{e}^{(1)} \otimes \cdots \otimes \mathbf{e}^{(n)}=\left\{x_{1} \otimes \cdots \otimes x_{n} \mid \forall i \in\{1, \ldots, n\}, x_{i} \in \mathbf{e}^{(i)}\right\}
$$

est une base orthonormée de $V_{1} \otimes \cdots \otimes V_{n}$ pour la norme hermitienne $\|\cdot\|_{\alpha}$.

Considérons maintenant une famille $\left(\bar{E}_{i}\right)_{i=1}^{n}$ de fibrés vectoriels adéliques. Soit $E=E_{1} \otimes \cdots \otimes E_{n}$. Si $\mathfrak{p}$ est une place finie, alors pour tout $i \in\{1, \ldots, n\}$, la norme 
d'indice $\mathfrak{p}$ de $\bar{E}_{i}$ correspond à un sous- $\widehat{\mathscr{\vartheta}}_{\mathfrak{p}}$-module libre $\mathcal{E}_{i, \mathfrak{p}}$ de $E_{i} \otimes_{K} \mathbb{C}_{\mathfrak{p}}$, où $\widehat{\vartheta}_{\mathfrak{p}}$ est l'anneau de valuation de $\mathbb{C}_{\mathfrak{p}}$. En effet, on a les relations

$$
\begin{gathered}
\mathcal{E}_{i, \mathfrak{p}}=\left\{x \in E \otimes_{K} \mathbb{C}_{\mathfrak{p}} \mid\|x\|_{\mathfrak{p}} \leqslant 1\right\}, \\
\forall x \in E_{i} \otimes_{K} \mathbb{C}_{\mathfrak{p}}, \quad\|x\|_{\mathfrak{p}}=\inf \left\{|a|_{\mathfrak{p}} \mid a \in \mathbb{C}_{\mathfrak{p}}, a^{-1} x \in \mathcal{E}_{i, \mathfrak{p}}\right\} .
\end{gathered}
$$

Les $\widehat{\vartheta}_{\mathfrak{p}}$-modules $\left(\mathcal{E}_{i, \mathfrak{p}}\right)_{i=1}^{n}$ déterminent une norme ultramétrique $\|\cdot\|_{\mathfrak{p}}$ sur l'espace tensoriel $E \otimes_{K} \mathbb{C}_{p}=E_{1, \mathbb{C}_{p}} \otimes \cdots \otimes E_{n, \mathbb{C}_{p}}$. En effet, on a

$$
\forall s \in E \otimes_{K} \mathbb{C}_{\mathfrak{p}}, \quad\|s\|_{\mathfrak{p}}=\inf \left\{|a|_{\mathfrak{p}} \mid a \in \mathbb{C}_{\mathfrak{p}}, a^{-1} s \in \mathcal{E}_{1, \mathfrak{p}} \otimes \cdots \otimes \mathcal{E}_{n, \mathfrak{p}}\right\} .
$$

Quitte à choisir une famille $\alpha=\left(\alpha_{v}\right)_{v \in \Sigma_{\infty}}$ de normes tensorielles d'ordre $n$, on obtient une structure de fibré vectoriel adélique $\left(\|\cdot\|_{v}\right)_{v \in \Sigma}$ sur $E$, où pour toute place infinie $v,\|\cdot\|_{v}=\|\cdot\|_{\alpha_{v}}$. Le fibré vectoriel adélique ainsi obtenu est noté $\bar{E}_{1} \otimes_{\alpha} \cdots \otimes_{\alpha} \bar{E}_{n}$, appelé le produit tensoriel de $\left(\bar{E}_{i}\right)_{i=1}^{n}$ du type $\alpha$. On dit que la famille $\alpha=\left(\alpha_{v}\right)_{v \in \Sigma_{\infty}}$ est hermitienne si toutes les normes tensorielles $\alpha_{v}$ sont hermitiennes.

Si les fibrés vectoriels adéliques $\bar{E}_{i}$ sont hermitiens et si la famille $\alpha$ est hermitienne, alors le fibré produit tensoriel $\bar{E}_{1} \otimes_{\alpha} \cdots \otimes_{\alpha} \bar{E}_{n}$ est lui-même hermitien, et s'identifie au produit tensoriel usuel $\bar{E}_{1} \otimes \cdots \otimes \bar{E}_{n}$. Mais il n'est pas vrai en général que le produit tensoriel $\bar{E}_{1} \otimes_{\alpha} \cdots \otimes_{\alpha} \bar{E}_{n}$ soit hermitien, même si toutes les normes tensorielles $\alpha_{v}$ sont hermitiennes. Le résultat suivant compare $\bar{E}_{1} \otimes_{\alpha} \cdots \otimes_{\alpha} \bar{E}_{n}$ aux produit des fibrés adéliques hermitiens, sachant que la famille $\alpha$ est hermitienne.

Proposition 2.1.15. - On suppose que $\left(\bar{E}_{i}\right)_{i=1}^{n}$ est une famille de fibrés vectoriels adéliques et que $\alpha=\left(\alpha_{v}\right)_{v \in \Sigma_{\infty}}$ est une famille de normes tensorielles hermitiennes. On pose $\left(E,\left(\|\cdot\|_{v}\right)_{v \in \Sigma}\right)=\bar{E}_{1} \otimes_{\alpha} \cdots \otimes_{\alpha} \bar{E}_{n}$ et

$$
\left(E,\left(\|\cdot\|_{v, \mathrm{~J}}\right)_{v \in \Sigma}\right):=\bigotimes_{i=1}^{n} \bar{E}_{i, \mathrm{~J}}, \quad\left(E,\left(\|\cdot\|_{v, \mathrm{~L}}\right)_{v \in \Sigma}\right):=\bigotimes_{i=1}^{n} \bar{E}_{i, \mathrm{~L}} .
$$

Alors, pour toute $v \in \Sigma_{\infty}$ et tout $x \in E \otimes_{K} \mathbb{C}_{v}, x \neq 0$, on a

$$
\begin{gathered}
\|x\|_{v, \mathrm{~L}} \leqslant\|x\|_{v} \leqslant\|x\|_{v, \mathrm{~J}} \\
\left|\log \|x\|_{v, \mathrm{~L}}-\log \|x\|_{v, \mathrm{~J}}\right| \leqslant \frac{3}{2} \sum_{i=1}^{n} \log \left(\operatorname{rg} E_{i}\right) .
\end{gathered}
$$

Démonstration. - Pour (35), voir [22] §5, la démonstration de la proposition 5.2. Dans la suite, on démontrera (36). Pour tout $i \in\{1, \ldots, n\}$, soit $\mathbf{e}^{(i)}$ une base orthonormée de $E_{i} \otimes_{K} \mathbb{C}_{v}$ pour la métrique $\|\cdot\|_{v, \text { Löwner. Soit }}$

$$
\mathbf{e}=\left\{s_{1} \otimes \cdots \otimes s_{n} \mid \forall i \in\{1, \ldots, n\}, s_{i} \in \mathbf{e}^{(i)}\right\} .
$$

C'est une base orthonormée de $E \otimes_{K} \mathbb{C}_{v}$ pour la norme $\|\cdot\|_{v, \mathrm{~L}}$. Pour tout élément $s=s_{1} \otimes \cdots \otimes s_{n} \in \mathbf{e}$, on a

$$
\log \|s\|_{v, \mathrm{~J}}=\sum_{i=1}^{n} \log \left\|s_{i}\right\|_{v, \mathrm{John}} \leqslant \sum_{i=1}^{n} \log \left\|s_{i}\right\|_{v, \text { Löwner }}+\log \operatorname{rg}\left(E_{i}\right)=\sum_{i=1}^{n} \log \left(\operatorname{rg} E_{i}\right) .
$$


Un élément général $x$ de $E \otimes_{K} \mathbb{C}_{v}$ s'écrit sous la forme $x=\sum_{s \in \mathbf{e}} a_{s} \cdot s$. D'après l'inégalité de Cauchy-Schwarz, on a

$$
\begin{aligned}
\log \|x\|_{v, \mathrm{~J}} & \leqslant \frac{1}{2} \log \sum_{s \in \mathbf{e}} a_{s}^{2}+\frac{1}{2} \log \sum_{s \in \mathbf{e}}\|s\|_{v, \mathrm{~J}}^{2} \\
& \leqslant \log \|x\|_{v, \mathrm{~L}}+\sum_{i=1}^{n} \log \left(\operatorname{rg} E_{i}\right)+\frac{1}{2} \log (\# \mathbf{e}) .
\end{aligned}
$$

D'où

$$
\sup _{0 \neq x \in E_{\mathbb{C}_{v}}}\left|\log \|x\|_{v, \mathrm{~L}}-\log \|x\|_{v, \mathrm{~J}}\right| \leqslant \sum_{i=1}^{n} \log \left(\operatorname{rg} E_{i}\right)+\frac{1}{2} \log (\# \mathbf{e})=\frac{3}{2} \sum_{i=1}^{n} \log \left(\operatorname{rg} E_{i}\right) .
$$

2.1.7. Produit tensoriel avec un fibré inversible adélique. - Soient $\bar{E}=$ $\left(E,\left(\|\cdot\|_{v}\right)_{v \in \Sigma}\right)$ un fibré vectoriel adélique et $\bar{M}=\left(M,\left(\|\cdot\|_{v}^{\prime}\right)_{v \in \Sigma}\right)$ un fibré inversible adélique sur Spec $K$. D'après [22, Proposition 5.2], pour toute famille de normes tensorielles $\alpha$, le produit tensoriel $\bar{E} \otimes_{\alpha} \bar{M}$ est isomorphe à $\left(E,\left(\|\cdot\|_{v} \cdot\|e\|_{v}^{\prime}\right)_{v \in \Sigma}\right)$, où $e \in M$ est un élément non-nul quelconque. Autrement, tous les produits tensoriels $\bar{E} \otimes_{\alpha} \bar{M}$ sont isomorphes. On désigne par $\bar{E} \otimes \bar{M}$ un produit tensoriel arbitraire de la forme $\bar{E} \otimes_{\alpha} \bar{M}$. Par définition, on a $\widehat{\mu}(\bar{E} \otimes \bar{M})=\widehat{\mu}(\bar{E})+\widehat{\mu}(\bar{M})$. On en déduit les égalités suivants :

$$
\widehat{\mu}_{\max }(\bar{E} \otimes \bar{M})=\widehat{\mu}_{\max }(\bar{E})+\widehat{\mu}(\bar{M}), \quad \widehat{\mu}_{\min }(\bar{E} \otimes \bar{M})=\widehat{\mu}_{\min }(\bar{E})+\widehat{\mu}(\bar{M})
$$

\subsection{Filtration et polygone de Harder-Narasimhan}

Dans cette section, on rappelle d'abord la notion de semi-stabilité et celle du drapeau de Harder-Narasimhan pour un fibré adélique hermitien, puis introduit la filtration de Harder-Narasimhan indexée par $\mathbb{R}$. Un lien explicite avec la notion classique est ensuite établi. Ensuite, on discute les polygones de Harder-Narasimhan dans le cas adélique.

2.2.1. Semi-stabilité et drapeau de Harder-Narasimhan. - On dit qu'un fibré adélique hermitien non-nul $\bar{E}$ est semi-stable si $\widehat{\mu}_{\max }(\bar{E})=\widehat{\mu}(\bar{E})$, ou de façon équivalente, $\widehat{\mu}_{\min }(\bar{E})=\widehat{\mu}(\bar{E})$.

D'après [22] proposition 5.3, il n'y a qu'un nombre fini de sous-espaces non-nuls $F$ de $E$ tel que $\widehat{\mu}(\bar{F})=\widehat{\mu}_{\max }(\bar{E})$. D'autre part, si $F_{1}$ et $F_{2}$ sont deux tels sous-espaces, alors la suite exacte courte

$$
0 \longrightarrow F_{1} \cap F_{2} \longrightarrow F_{1} \oplus F_{2} \longrightarrow F_{1}+F_{2} \longrightarrow 0
$$

implique que

$$
\begin{aligned}
\widehat{\operatorname{deg}}_{n}\left(\overline{F_{1}+F_{2}}\right) & \geq \widehat{\operatorname{deg}}_{n}\left(\bar{F}_{1}\right)+\widehat{\operatorname{deg}}_{n}\left(\bar{F}_{2}\right)-\widehat{\operatorname{deg}}_{n}\left(\overline{F_{1} \cap F_{2}}\right) \\
& \geqslant \widehat{\mu}_{\text {max }}(\bar{E})\left(\operatorname{rg} F_{1}+\operatorname{rg} F_{2}-\operatorname{rg}\left(F_{1} \cap F_{2}\right)\right),
\end{aligned}
$$


et on obtient donc $\widehat{\mu}\left(\overline{F_{1}+F_{2}}\right)=\widehat{\mu}_{\max }(\bar{E})$. Cela montre l'existence d'un plus grand sous-espace $E_{\text {des }}$ de $E$ tel que $\widehat{\mu}\left(\bar{E}_{\text {des }}\right)=\widehat{\mu}_{\text {max }}(\bar{E})$. Par définition, $\bar{E}_{\text {des }}$ est semi-stable, et $\bar{E}_{\mathrm{des}}=\bar{E}$ si et seulement si $\bar{E}$ est semi-stable. Si ce n'est pas le cas, on dit que $\bar{E}_{\text {des }}$ est le sous-fibré adélique hermitien qui déstabilise $\bar{E}$. Par récurrence on obtient un drapeau de $E$ :

$$
E=E_{0} \supsetneq E_{1} \supsetneq \cdots \supsetneq E_{d}=0
$$

tel que $\overline{E_{i} / E_{i+1}}=\left(\overline{E / E_{i+1}}\right)_{\text {des }}$ quel que soit $i \in\{0, \ldots, d-1\}$. Ce drapeau est appelé le drapeau de Harder-Narasimhan de $\bar{E}$. Par définition, les sous-quotients $\overline{E_{i} / E_{i+1}}$ sont semi-stables. En outre, si on note $\mu_{i}=\widehat{\mu}\left(\overline{E_{i} / E_{i+1}}\right)$, alors on a

$$
\widehat{\mu}_{\min }(\bar{E})=\mu_{0}<\mu_{1}<\cdots<\mu_{d-1}=\widehat{\mu}_{\max }(\bar{E}) .
$$

Les nombres $\mu_{i}$ sont appelés les pentes successives de $\bar{E}$.

2.2.2. Filtration de Harder-Narasimhan. - La donnée du drapeau de HarderNarasimhan (38) et des pentes successives (39) déterminent une $\mathbb{R}$-filtration notée $\mathcal{F}^{\bar{E}}$ de $E$ telle que (cf. $§ 1.2 .1$ infra)

$$
\mathcal{F}_{s}^{\bar{E}} E=\bigcup_{\substack{0 \leqslant i<d \\ \mu_{i} \geqslant s}} E_{i},
$$

appelée la filtration de Harder-Narasimhan de $\bar{E}$. Par définition, on a

$$
\lambda_{\min }\left(E_{K}, \mathcal{F}^{\bar{E}}\right)=\widehat{\mu}_{\min }(\bar{E}) \quad \text { et } \quad \lambda_{\max }\left(E_{K}, \mathcal{F}^{\bar{E}}\right)=\widehat{\mu}_{\max }(\bar{E}),
$$

où $\lambda_{\min }$ et $\lambda_{\max }$ sont définies dans (3).

Si $\bar{E}=0$, alors par convention $\mathcal{F}^{0}$ est l'unique filtration de l'espace nul. Les égalités dans (40) sont encore valides.

Proposition 2.2.1. - Pour tout $s \in \mathbb{R}, \widehat{\mu}_{\min }\left(\overline{\mathcal{F}_{s}^{\bar{E}} E}\right) \geqslant s$.

Démonstration. - La proposition est évidente lorsque $\mathcal{G}_{s}^{\bar{E}} E=0$. Dans la suite, on suppose que $\mathcal{G}_{s}^{\bar{E}} E$ est non-nul, d'où l'espace $E$ lui-même est non-nul. Soit le drapeau de Harder-Narasimhan de $\bar{E}$ comme dans (38). Soit $i \in\{0, \ldots, d\}$ tel que $\mathcal{F}_{s}^{\bar{E}} E=E_{i}$. Comme $\mathcal{F}_{s}^{\bar{E}} E$ est non-nul, on a $i<d$. Le drapeau $E_{i} \supsetneq E_{i+1} \supsetneq \cdots \supsetneq E_{d}$ est le drapeau de Harder-Narasimhan de $E_{i}$. Donc $\widehat{\mu}_{\min }\left(E_{i}\right)=\mu_{i} \geqslant s$.

Proposition 2.2.2. - Soient $\bar{E}$ et $\bar{F}$ deux fibrés adéliques hermitiens. Si $\varphi: E \rightarrow F$ est une application $K$-linéaire, alors $\varphi(E)$ est contenue dans $\mathcal{F}_{\widehat{\mu}_{\min }}^{\bar{F}}(\bar{E})-h(\varphi) F$.

Démonstration. - Le cas où $\varphi=0$ est trivial. On suppose alors que $\varphi$ est non-nul. Soit $F=F_{0} \supsetneq F_{1} \supsetneq \cdots \supsetneq F_{m}=0$ le drapeau de Harder-Narasimhan de $F$. Soit $i$ le plus grand indice dans $\{0, \ldots, m\}$ tel que $\varphi(E) \subset F_{i}$. Comme $\varphi$ est non-nul, on a $i<m$. Soit $\psi$ l'homomorphisme composé $E \stackrel{\varphi}{\longrightarrow} F_{i} \longrightarrow F_{i} / F_{i+1}$ où la dernière 
flèche est la projection canonique. L'homomorphisme $\psi$ est non-nul car l'image de $E$ $\operatorname{par} \varphi$ n'est pas contenue dans $F_{i+1}$. Par l'inégalité de pentes $(24)$, on obtient

$$
\widehat{\mu}_{\min }(\bar{E}) \leqslant \widehat{\mu}_{\max }\left(\overline{F_{i} / F_{i+1}}\right)+h(\psi) \leqslant \widehat{\mu}\left(\overline{F_{i} / F_{i+1}}\right)+h(\varphi) .
$$

On en déduit $F_{i}=\mathcal{F}_{\widehat{\mu}\left(\overline{F_{i} / F_{i+1}}\right)}^{\bar{F}} F \subset \mathcal{F}_{\widehat{\mu}_{\min }}^{\bar{F}}(\bar{E})-h(\varphi) F$.

Corollaire 2.2.3. - Pour tout fibré adélique hermitien non-nul $\bar{E}$, on a

$$
\mathcal{F}_{s}^{\bar{E}} E=\sum_{\substack{0 \neq F \subset E \\ \widehat{\mu}_{\min }(\bar{F}) \geqslant s}} F .
$$

Démonstration. - D'une part, la proposition 2.2.2 montre que, si $F \subset E$ est un sousespace non-nul tel que $\widehat{\mu}_{\min }(\bar{F}) \geqslant s$, alors $F$ est contenu dans $\mathcal{F}_{s}^{\bar{E}} E$, sachant que l'application d'inclusion de $F$ dans $E$ est de hauteur nulle. D'autre part, la proposition 2.2.1 affirme que $\widehat{\mu}_{\min }\left(\overline{\mathcal{F}_{s}^{\bar{E}} E}\right) \geqslant s$. Donc $\mathcal{F}_{s}^{\bar{E}} E$ est en fait le plus grand sous-espace $G$ de $E$ tel que $\widehat{\mu}_{\min }(\bar{G}) \geqslant s$, d'où $(41)$.

La proposition suivante compare les filtrations de Harder-Narasimhan de deux fibrés adéliques hermitiens.

Proposition 2.2.4. - Soient $\bar{E}$ et $\bar{F}$ deux fibrés adéliques hermitiens sur Spec $K$. Soit $\varphi: E \rightarrow F$ une application K-linéaire. Alors, pour tout $s \in \mathbb{R}$, l'image de $\mathcal{F}_{s}^{\bar{E}} E$ par $\varphi$ est contenue dans $\mathcal{F}_{s-h(\varphi)}^{\bar{F}} F$. Autrement dit, $\lambda_{\mathscr{F}^{\bar{F}}}(\varphi(x)) \geqslant \lambda_{\mathscr{G}^{\bar{E}}}(x)-h(\varphi)$ quel que soit $x \in E$.

Démonstration. - D'après la proposition 2.2.1, $\widehat{\mu}_{\min }\left(\overline{\mathcal{F}_{s}^{\bar{E}} E}\right) \geqslant s$. On obtient donc $\varphi\left(\mathcal{F}_{s}^{\bar{E}} E\right) \subset \mathcal{F}_{s-h(\varphi)}^{\bar{F}} F$ compte tenu de la proposition 2.2.2.

DÉFInition 2.2.5. - On désigne par $\nu_{\bar{E}}$ la mesure $\nu_{\mathcal{G}} \bar{E}$, appelée la mesure associée $\grave{a} \bar{E}$. Si $E$ est non-nul, alors $\nu_{\bar{E}}$ est une mesure de probabilité.

Corollaire 2.2.6. - Si $\bar{E}$ et $\bar{E}^{\prime}$ sont deux fibrés adéliques hermitiens et si $\varphi: E \rightarrow$ $E^{\prime}$ est un isomorphisme d'espaces vectoriels sur $K$, alors $\tau_{h(\varphi)} \nu_{\bar{E}^{\prime}} \succ \nu_{\bar{E}}$.

Démonstration. - C'est une conséquence de la proposition 2.2.4 et du lemme 1.2.6. 
2.2.3. Polygone de Harder-Narasimhan. - Étant donné un fibré adélique hermitien non-nul $\bar{E}$, on désigne par $\mathscr{P}_{\bar{E}}$ la fonction définie sur $[0,1]$ dont le graphe est le bord supérieur de l'enveloppe convexe des points $(\operatorname{rg} F / \operatorname{rg} E, \widehat{\mu}(\bar{F}))$, où $F$ parcourt tous les sous-espaces de $E$. La fonction $\mathscr{P}_{\bar{E}}$ est un polygone, appelé le polygone de Harder-Narasimhan (normalisé) de $\bar{E}$. Si le drapeau de HarderNarasimhan de $\bar{E}$ est (38) et si les pentes successives de $\bar{E}$ sont comme dans (39), alors les sommets de $\mathscr{P}_{\bar{E}}$ sont de coordonnés $\left(\operatorname{rg} E_{i} / \operatorname{rg} E, \widehat{\mu}\left(\bar{E}_{i}\right)\right)$, et les pentes de $\mathscr{P}_{\bar{E}}$ coïncident avec les $\mu_{i}$. On en déduit donc la proposition suivante :

Proposition 2.2.7. - Le polygone de Harder-Narasimhan normalisé de $\bar{E}$ coïncide avec $\mathscr{P}\left(\nu_{\bar{E}}\right)$, le polygone associé à la mesure $\nu_{\bar{E}}$ défini dans \$1.2.5. En particulier,

$$
\begin{gathered}
\widehat{\mu}(\bar{E})=\mathscr{P}_{\bar{E}}(1)=\int_{\mathbb{R}} x \nu_{\bar{E}}(\mathrm{~d} x), \\
\widehat{\mu}_{\max }(\bar{E})=\mathscr{P}_{\bar{E}}^{\prime}(0)=\sup \left(\operatorname{supp} \nu_{\bar{E}}\right), \quad \widehat{\mu}_{\min }(\bar{E})=\mathscr{P}_{\bar{E}}^{\prime}(1)=\inf \left(\operatorname{supp} \nu_{\bar{E}}\right) .
\end{gathered}
$$

Remarque 2.2.8. - Classiquement le polygone de Harder-Narasimhan de $\bar{E}$ est par définition la fonction $\widetilde{\mathscr{P}}_{\bar{E}}$ définie sur $[0, \mathrm{rg} E]$ dont le graphe est le bord supérieur de l'enveloppe convexe des points $\left(\operatorname{rg} F, \widehat{\operatorname{deg}}_{n}(\bar{F})\right)$, où $F$ parcourt tous les sous-espaces vectoriels de $E$. Autrement dit, on a la relation $\mathscr{P}_{\bar{E}}(t)=\widetilde{\mathscr{P}}_{\bar{E}}(t \mathrm{rg} E) / \mathrm{rg} E$. La version normalisée du polygone de Harder-Narasimhan a deux avantages. Premièrement, les valeurs de $\mathscr{P}_{\bar{E}}$ représente différentes pentes de $\bar{E}$, qui sont plus couramment utilisées que les degrés dans la méthode des pentes. Deuxièmement, les polygones de HarderNarasimhan normalisés sont tous définis sur le même intervalle $[0,1]$, cela donne la possibilité de comparer les polygones de différents fibrés adéliques hermitiens. La proposition 2.2.7 nous permet d'utiliser les mesures à étudier les polygones de HarderNarasimhan.

Corollaire 2.2.9. - Si $\bar{E}$ et $\bar{E}^{\prime}$ sont deux fibrés adéliques hermitiens et si $\varphi: E \rightarrow$ $E^{\prime}$ est un isomorphisme d'espaces vectoriels sur $K$, alors $\mathscr{P}_{\bar{E}}(t) \leqslant \mathscr{P}_{\bar{E}^{\prime}}(t)+h(\varphi) t$.

Démonstration. - On a $\mathscr{P}\left(\tau_{h(\varphi)} \nu_{\bar{E}^{\prime}}\right) \geqslant \mathscr{P}\left(\nu_{\bar{E}}\right)$, compte tenu de la proposition 1.2 .7 et du corollaire 2.2.6. D'après $(6), \mathscr{P}\left(\tau_{h(\varphi)} \nu_{\bar{E}^{\prime}}\right)(t)=\mathscr{P}\left(\nu_{\bar{E}^{\prime}}\right)(t)+h(\varphi) t=\mathscr{P}_{\bar{E}^{\prime}}(t)+h(\varphi) t$. D'autre part, $\mathscr{P}\left(\nu_{\bar{E}}\right)=\mathscr{P}_{\bar{E}}$. On obtient donc $\mathscr{P}_{\bar{E}}(t) \leqslant \mathscr{P}_{\bar{E}^{\prime}}(t)+h(\varphi) t$.

Remarque 2.2.10. - Dans [22, §5.2], Gaudron a défini le polygone de HarderNarasimhan et les pentes successives pour un fibré vectoriel adélique général. Étant donné un fibré vectoriel adélique non-nul $\bar{E}$, il a défini le polygone de HarderNarasimhan de $\bar{E}$ comme la fonction réelle $\widetilde{\mathscr{P}}_{\bar{E}}$ sur $[0, \mathrm{rg} E]$ qui délimite l'enveloppe convexe de l'ensemble $\left\{\left(\operatorname{rg}(F), \widehat{\operatorname{deg}}_{n}(\bar{F})\right) \mid F \subset E\right\}$. Pour $i \in\{1, \ldots, \operatorname{rg} E\}$, la $i^{\text {ème }}$ pente de $\bar{E}$ est définie comme le nombre réel $\widehat{\mu}_{i}(\bar{E}):=\widetilde{\mathscr{P}}_{\bar{E}}(i)-\widetilde{\mathscr{P}}_{\bar{E}}(i-1)$. On peut aussi définir une version normalisé du polygone de Harder-Narasimhan de $\bar{E}$ en posant $\mathscr{P}_{\bar{E}}(t)=\widetilde{\mathscr{P}}_{\bar{E}}(t \mathrm{rg} E) / \mathrm{rg} E$. Cependant, comme on n'a plus l'égalité 
$\widehat{\mu}_{\mathrm{rg}(E)}(\bar{E})=\widehat{\mu}_{\min }(\bar{E})$, le polygone associée à la filtration de la forme (41) ne coïncide pas forcément avec le polygone de Harder-Narasimhan normalisé de $\bar{E}$. Donc on ne peut pas utiliser directement les mesures de probabilité à étudier les polygones de Harder-Narasimhan dans ce cas général. Néanmoins, si on désigne par $\bar{E}_{\mathrm{J}}$ et $\bar{E}_{\mathrm{L}}$ les fibrés vectoriels hermitiens dont les espaces vectoriel sous-jacent sont $E$ et dont les métriques sont respectivement les métriques hermitiennes de John et de Löwner associées aux métriques de $\bar{E}$, alors on a les relations

$$
\mathscr{P}_{\bar{E}_{\mathrm{J}}} \leqslant \mathscr{P}_{\bar{E}} \leqslant \mathscr{P}_{\bar{E}_{\mathrm{L}}} .
$$

C'est une conséquence de la proposition 2.1.5. En outre, la hauteur de l'application d'identité $\bar{E}_{\mathrm{L}} \rightarrow \bar{E}_{\mathrm{J}}$ est bornée supérieurement par $\log (\operatorname{rg} E)$. Par conséquent, on a

$$
\left|\mathscr{P}_{\bar{E}_{\mathrm{L}}}(t)-\mathscr{P}_{\bar{E}_{\mathrm{J}}}(t)\right| \leqslant t \log (\mathrm{rg} E)
$$

compte tenu du corollaire 2.2.9. Cette estimation nous permettra d'obtenir le comportement asymptotique des polygones de Harder-Narasimhan dans le cadre de fibrés vectoriels adéliques.

\subsection{Cas de corps de fonctions}

Il est bien connu que les corps de fonctions et les corps de nombres son très similaires. Étant donné un corps $k$. Un corps de fonctions est une extension finie du corps $k(T)$ des fractions rationnelles à une variable. Un tel corps peut s'écrire comme le corps des fonctions méromorphes sur une courbe projective régulière $C$ définie sur $k$. La théorie des fibrés vectoriels adéliques sur un corps de fonctions est aussi développée dans [22]. Dans cette section, on rappelle la notion de fibré vectoriel adélique et on discute les filtrations de Harder-Narasimhan dans ce cadre. On fixe un corps de fonctions $K^{\prime}=k(C)$, où $C$ est un schéma projectif intègre $\operatorname{sur} \operatorname{Spec} k$, supposé être régulier et de dimension 1.

2.3.1. Fibré vectoriel adélique sur un corps de fonctions. - Soit $\Sigma^{\prime}$ l'ensemble des places de $K^{\prime}$, qui s'identifie à l'ensemble des points fermés de $C$. Toutes les places dans $\Sigma^{\prime}$ sont non-archimédiennes. Pour chaque $x \in \Sigma^{\prime}$, on désigne par $n_{x}$ l'entier $[k(x): k]$, où $k(x)$ est le corps résiduel de $C$ en $x$. Soit en outre $v_{x}$ la valuation discrète de $K^{\prime}$ correspondant à $x$. La version logarithmique de la formule de produit pour $K^{\prime}$ s'énonce comme

$$
\forall f \in K^{\prime \times}, \quad \sum_{x \in \Sigma^{\prime}} n_{x} v_{x}(f)=0 .
$$

Soit $|\cdot|_{x}$ la valeur absolue sur $K^{\prime}$ telle que $|f|_{x}=e^{-v_{x}(f)}$ quel que soit $f \in K^{\prime \times}$. On désigne par $K_{x}^{\prime}$ le complété de $K^{\prime}$ pour la valeur absoule $|\cdot|_{x}$ et par $\mathbb{C}_{x}$ le complété d'une clôture algébrique de $K_{x}^{\prime}$, où la valeur absolue $|\cdot|_{x}$ s'étend de façon unique. 
Remarque 2.3.1. - Dans [22], l'auteur a choisi de travailler dans le cas où le corps $k$ est fini. Dans ce cas-là, il y a un choix "canonique" de la métrique $|\cdot|_{x}$ en posant $|\cdot|_{x}=(\# k)^{-v_{x}(\cdot)}$. Mais le changement de valeurs absolues conduit à une constante supplémentaire (strictement positive) devant les degrés d'Arakelov et les pentes, qui est en fait anodin. Donc les résultats et les démonstrations dans loc. cit. sont valables pour $k$ quelconque, quitte à désigner une valeur absolue de l'uniformisante.

DÉFInition 2.3.2. - On appelle fibré vectoriel adélique sur Spec $K^{\prime}$ toute donnée $\bar{E}=\left(E,\left(\|\cdot\|_{x}\right)_{x \in \Sigma^{\prime}}\right)$ d'un espace vectoriel $E$ de rang fini sur $K^{\prime}$ et d'une famille de normes ultramétriques $\|\cdot\|_{x} \operatorname{sur} E \otimes_{K^{\prime}} \mathbb{C}_{x}$, soumise aux conditions suivante :

1) Il existe une base $\left(s_{1}, \ldots, s_{r}\right)$ de $E$ sur $K^{\prime}$ et une partie finie $S$ de $\Sigma^{\prime}$ telle que $\left\|a_{1} s_{1}+\cdots+a_{r} s_{r}\right\|_{x}=\max \left(\left|a_{1}\right|_{x}, \ldots,\left|a_{r}\right|_{x}\right)$ quels que soient $x \in \Sigma^{\prime} \backslash S$ et $\left(a_{1}, \ldots, a_{r}\right) \in \mathbb{C}_{x}^{r}$.

2) Pour tout $x \in \Sigma^{\prime}$, la norme $\|\cdot\|_{x}$ est invariante sous l'action de $\operatorname{Gal}\left(\mathbb{C}_{x} / K_{x}^{\prime}\right)$. Plus précisément, si $\left(e_{1}, \ldots, e_{r}\right)$ est une base de $E_{K_{x}^{\prime}}$ sur $K_{x}^{\prime}$ et si $a_{1}, \ldots, a_{r}$ sont des éléments dans $\mathbb{C}_{x}$, alors $\left\|\tau\left(a_{1}\right) e_{1}+\cdots+\tau\left(a_{r}\right) e_{r}\right\|_{x}=\left\|a_{1} e_{1}+\cdots+a_{r} e_{r}\right\|_{x}$ quel que soit $\tau \in \operatorname{Gal}\left(\mathbb{C}_{x} / K_{x}^{\prime}\right)$.

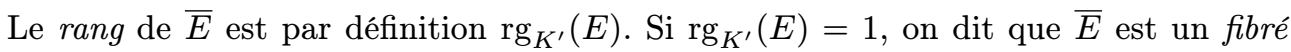
inversible adélique.

Comme toute place de $K^{\prime}$ est non-archimédienne, $\bar{E}$ est automatiquement "hermitien". Si $r=\operatorname{rg}_{K^{\prime}} E$ et si $\left(s_{1}, \ldots, s_{r}\right)$ est une base de $E$ sur $K^{\prime}$, alors le degré d'Arakelov de $E$ et sa version normalisée sont respectivement

$$
\widehat{\operatorname{deg}}(\bar{E})=-\sum_{x \in \Sigma^{\prime}} n_{x} \log \left\|s_{1} \wedge \cdots \wedge s_{r}\right\|_{x} \quad \text { et } \quad \widehat{\operatorname{deg}}_{n}(\bar{E})=\frac{1}{\operatorname{deg}(C)} \widehat{\operatorname{deg}}(\bar{E}) .
$$

Cette définition ne dépend pas du choix de la base $\left(s_{1}, \ldots, s_{r}\right)$, grâce à la formule de produit (43). L'avantage du procédé de normalisation est que, la fonction $\widehat{\operatorname{deg}}_{n}$ est invariant par toute extension finie du scalaire. Les opérations algébriques sont naturellement définies pour les fibrés vectoriels adéliques sur Spec $K^{\prime}$. En particulier, le déterminant $\operatorname{dét}(\bar{E}):=\Lambda^{\mathrm{rg} E} \bar{E}$ satisfait à l'égalité $\widehat{\operatorname{deg}}(\operatorname{dét}(\bar{E}))=\widehat{\operatorname{deg}}(\bar{E})$. La caractéristique d'Euler-Poincaré est définie comme

$$
\chi(\bar{E})=\widehat{\operatorname{deg}}(\bar{E})+\operatorname{rg} E(1-g),
$$

où $g$ est le genre de $C$.

Remarque 2.3.3. - Si $\mathcal{E}$ est un $\theta_{C}$-module localement libre de rang fini, et si $E=$ $\mathcal{E}_{K^{\prime}}$, alors la structure de $\theta_{C^{-}}$-module sur $\mathcal{E}$ définit naturellement une famille de ultranormes $\|\cdot\|_{x}$ sur les $E \otimes_{K^{\prime}} \mathbb{C}_{x}$ où $x \in \Sigma^{\prime}$. On désigne par $\widehat{\theta}_{x}$ l'anneau de valuation dans $\mathbb{C}_{x}$. La norme $\|\cdot\|_{x}$ est définie comme

$$
\|s\|_{x}=\inf \left\{|a|_{x} \mid a \in \mathbb{C}_{x}, s \in a\left(\mathcal{E}_{x} \otimes_{\vartheta_{C, x}} \widehat{\vartheta}_{x}\right)\right\} .
$$

On obtient ainsi un fibré vectoriel adélique $\bar{E} \operatorname{sur} \operatorname{Spec} K^{\prime}$, et on a la relation $\widehat{\operatorname{deg}}(\bar{E})=$ $\operatorname{deg}(\mathcal{E})$. 
Réciproquement, pour toute partie finie non-vide $S$ de $\Sigma^{\prime}, U=C \backslash S$ est un schéma affine. On désigne par $\theta_{U}$ son anneau. Étant donné un fibré vectoriel adélique $\bar{E}$ sur Spec $K^{\prime}$, il s'avère que l'ensemble

$$
\mathcal{E}_{U}:=\left\{s \in E \mid \forall x \in U \cap \Sigma^{\prime},\|s\|_{x} \leqslant 1\right\}
$$

est un $\theta_{U}$-module projectif de type fini. On suppose que les métriques de $\bar{E}$ sont discrètes. Pour tout $x \in U \cap \Sigma^{\prime}$, la norme sur $E$ induit par la structure de $\Theta_{C, x}$-module sur $\mathcal{E}_{U, x}$ coïncide avec $\|\cdot\|_{x}$. Si $U \subset V$ sont deux ouvert affine de $C$, alors l'application d'inclusion $\mathscr{E}_{V} \rightarrow \mathcal{E}_{U}$ est compatible à l'homomorphisme canonique $\theta_{V} \rightarrow \theta_{U}$. Par recollement on obtient un $\theta_{C}$-module localement libre de rang fini $\mathcal{E}$ tel que $\mathcal{E}_{K}=E$ et que, pour tout $x \in \Sigma$, la norme induite par la structure de $\theta_{C, x}$-module sur $\mathcal{E}_{x}$ s'identifie à $\|\cdot\|_{x}$.

2.3.2. Pentes, filtration et polygone de Harder-Narasimhan. - Les constructions dans la section précédente, notamment celles de pente, de filtration et polygone de Harder-Narasimhan sont valables pour tout fibré vectoriel adélique nonnul sur Spec $K^{\prime}$. Il suffit de remplacer dans $§ 2.1$ et $\S 2.2$ les occurrences de l'expression "fibré adélique hermitien" par "fibré vectoriel adélique" et celles du symboles " $K$ " par " $K^{\prime \prime}$.

Dans le cadre de la géométrie algébrique sur $C$, on est plus habitué à la version non-normalisée du degré et des pentes. Soit $\mathcal{E}$ un fibré vectoriel non-nul sur $C$, qui correspond à un fibré vectoriel adélique $\overline{\mathcal{E}}_{K^{\prime}}$ sur Spec $K^{\prime}$. On désigne par $\mu(\mathcal{E})$ la pente de $\mathcal{E}$, qui est le quotient $\operatorname{deg}(\mathcal{E}) / \operatorname{rg}(\mathcal{E})$. Par définition on a la relation $\mu(\mathcal{E})=$ $\operatorname{deg}(C) \widehat{\mu}\left(\overline{\mathscr{E}}_{K^{\prime}}\right)$. Les faits sur les fibrés adéliques que l'on a présentés conduisent à des énoncés analogues dans ce cadre qui correspond aux résultats classiques concernant les fibrés vectoriels sur $C$. Pour faciliter les lecteurs, on résume quelques constructions et résultats.

Étant donné un fibré vectoriel non-nul $\mathcal{E}$ sur $C$. La pente maximale et la pente minimale de $\mathcal{E}$ existent. On rappelle que la pente maximale $\mu_{\max }(\mathcal{E})$ est la valeur maximale des pentes des sous-fibrés vectoriels de $\mathcal{E}$; et la pente minimale $\mu_{\min }(\mathcal{E})$ est la valeur minimale des pentes des quotients localement libres de $\mathcal{E}$. Le fibré vectoriel $\mathcal{E}$ est dit semi-stable si $\mu(\mathcal{E})=\mu_{\max }(\mathcal{E})$, ou de façon équivalente, $\mu(\mathcal{E})=\mu_{\min }(\mathcal{E})$.

Il existe un unique drapeau $\mathcal{E}=\varepsilon_{0} \supsetneq \varepsilon_{1} \supsetneq \cdots \supsetneq \varepsilon_{d}=0$ tel que les sous-quotients $\varepsilon_{i} / \mathcal{E}_{i+1}$ soient semi-stables et que $\mu\left(\varepsilon_{0} / \mathcal{E}_{1}\right)<\cdots<\mu\left(\mathcal{E}_{d-1} / \mathcal{E}_{d}\right)$. Ce drapeau induit par restriction à la fibre générique un drapeau $\mathcal{E}_{K^{\prime}}=\mathcal{E}_{0, K^{\prime}} \supsetneq \mathcal{E}_{1, K^{\prime}} \supsetneq \cdots \supsetneq \mathcal{E}_{d, K^{\prime}}=0$ d'espaces vectoriels sur $K^{\prime}$. On en déduit donc (cf. $\S 1.2 .1 \mathrm{infra}$ ) une filtration de $\mathcal{E}_{K^{\prime}}$, appelée la filtration de Harder-Narasimhan de $\mathcal{E}$. On désigne par $\mathscr{P}_{\mathscr{E}}$ le polygone normalisé correspondant.

Comme expliqué plus haut dans la remarque 2.3.3, le fibré vectoriel $\mathcal{E}$ détermine un fibré vectoriel adélique $\overline{\mathcal{E}}_{K^{\prime}}$. On a les relations

$$
\widehat{\mu}_{\max }\left(\overline{\mathscr{E}}_{K^{\prime}}\right)=\frac{\mu_{\max }(\mathcal{E})}{\operatorname{deg}(C)}, \quad \widehat{\mu}_{\min }\left(\overline{\mathscr{E}}_{K^{\prime}}\right)=\frac{\mu_{\min }(\mathcal{E})}{\operatorname{deg}(C)} \quad \text { et } \quad \mathscr{P}_{\overline{\mathscr{E}}_{K^{\prime}}}=\frac{\mathscr{P}_{\mathscr{E}}}{\operatorname{deg}(C)} .
$$


Dans le cadre de la géométrie relative, les inégalités de pentes sont plus simples. Notamment, si $\varphi: \mathcal{E} \rightarrow \mathcal{F}$ est un homomorphisme non-nul de fibrés vectoriels sur $C$, alors $\mu_{\min }(\mathcal{E}) \leqslant \mu_{\max }(\mathcal{F})$. Si $\varphi$ est injectif, alors $\mu_{\max }(\mathcal{E}) \leqslant \mu_{\max }(\mathcal{F})$. Si $\varphi$ est surjectif, alors $\mu_{\min }(\mathcal{E}) \leqslant \mu_{\min }(\mathcal{F})$. 



\section{CHAPITRE 3}

\section{CONVERGENCE DES POLYGONES}

Dans ce chapitre, on établit la convergence uniforme des polygones associés à une algèbre graduée dont chaque composante homogène est munie d'une $\mathbb{R}$-filtration. Deux stratégies radicalement différentes sont proposées. La première est inspirée par un travail de Faltings et Wüstholz [20] qui remonte à l'idée très classique de séries de Poincaré. Cette approche, qui permet de calculer explicitement la limite des polygones, se restreint cependant au cas d'algèbre $\mathbb{N}^{2}$-bigraduée, c'est-à-dire au cas où les filtrations sont induites par une autre graduation. Ce cas particulier est loin d'être suffisant pour les applications arithmétiques car déjà les pentes successives d'un fibré adélique hermitien, qui décrivent les points de saut de sa filtration de Harder-Narasimhan, ne sont pas nécessairement des entiers, autrement dit, en général la filtration de Harder-Narasimhan ne peut pas être induite par une $\mathbb{Z}$-graduation. D'autre part, même si dans certains cas particuliers les pentes successives sont des entiers, il est peu plausible d'espérer que la structure d'algèbre de l'algèbre qui nous intéresse soit compatible aux graduations induites par les filtrations de HarderNarasimhan. L'une des difficultés majeures pour étendre cette approche au cas général est l'absence de finitude de l'algèbre bigraduée associée à une algèbre graduée munie des filtrations. Pour surmonter cette difficulté, on adopte la deuxième stratégie qui consiste à montrer que les mesures associées aux filtrations que l'on considère vérifient une sorte de sur-additivité. En s'appuyant sur les généralisations du lemme de Fekete établies dans $\S 1.3$, on obtient la convergence vague des mesures dilatées et en déduit la convergence uniforme des polygones normalisés.

On fixe dans ce chapitre un corps $k$. Toutes les algèbres sont supposées être sur $k$.

\subsection{Cas de modules bigradués}

Dans cette section, on présente la théorie des séries de Poincaré à deux variables qui est similaire à la théorie classique (cf. [14] VIII §4). On étudie ensuite le comportement asymptotique de ces séries de Poincaré et en déduit le comportement asymptotique des polygones associés. 
On entend par algèbre bigraduée toute $k$-algèbre commutative $\mathbb{N}^{2}$-graduée. Autrement dit, une algèbre bigraduée $A$ est une somme directe $A=\bigoplus_{(n, d) \in \mathbb{N}^{2}} A_{n, d}$ d'espaces vectoriels sur $k$, munie d'une structure de $k$-algèbre unifère telle que $A_{n, d} A_{n^{\prime}, d^{\prime}} \subset A_{n+n^{\prime}, d+d^{\prime}}$.

Pour munir une algèbre de polynôme $k\left[T_{1}, \ldots, T_{m}\right]$ d'une structure d'algèbre bigraduée, il suffit d'assigner une application $f:\{1, \ldots, m\} \rightarrow \mathbb{N}^{2}$. Dans ce cas-là, l'élément $T_{i}$ est homogène de bidegré $f(i)$. Une telle algèbre est notée $k[f]$.

Étant donnée une algèbre bigraduée $A$. On appelle $A$-module bigradué tout $A$ module $M$ muni d'une $\mathbb{Z}^{2}$-graduation en espaces vectoriels sur $k$ telle que, pour tout $(n, d) \in \mathbb{N}^{2}$ et tout $\left(n^{\prime}, d^{\prime}\right) \in \mathbb{Z}^{2}$, on ait $A_{n, d} M_{n^{\prime}, d^{\prime}} \subset M_{n+n^{\prime}, d+d^{\prime}}$. À partir de $M$, on peut construire des nouveaux $A$-modules bigradués en décalant les composantes homogènes : pour tout $(n, d) \in \mathbb{Z}^{2}$, on désigne par $M(n, d)$ le $A$-module bigradué tel que $M(n, d)_{n^{\prime}, d^{\prime}}=M_{n+n^{\prime}, d+d^{\prime}}$. En outre, les $A$-modules bigradués et les homomorphismes homogènes forment une catégorie abélienne. Cela nous permet de considérer les suites exactes de $A$-modules bigradués.

Si $A$ est une algèbre bigraduée de type fini, alors elle est engendrée par un nombre fini d'éléments homogènes $a_{1}, \ldots, a_{m}$. Soit $f:\{1, \ldots, m\} \rightarrow \mathbb{N}^{2}$ l'application qui envoie $i$ en le bidegré de $a_{i}$. Alors l'homomorphisme surjectif de $k$-algèbres de $k[f] \cong$ $k\left[T_{1}, \ldots, T_{m}\right]$ vers $A$ qui envoie $T_{i}$ en $a_{i}$ est homogène. Tout $A$-module bigradué peut donc être considéré comme un $k[f]$-module bigradué, qui est de type fini si $M$ est un $A$-module de type fini.

On renvoie les lecteurs à [13] II $§ 11$ pour une présentation plus complète des algèbres graduées et des modules gradués.

3.1.1. Séries de Poincaré à deux variables. - Soient $f=\left(f_{1}, f_{2}\right)$ une application de $\{1, \ldots, m\}$ vers $\mathbb{N}^{2}$ et $M$ un $k[f]$-module bigradué de type fini. On appelle série de Poincaré de $M$ l'élément $P_{M} \in \mathbb{Z} \llbracket X, Y \rrbracket\left[X^{-1}, Y^{-1}\right]$ défini par

$$
P_{M}=\sum_{(n, d) \in \mathbb{Z}^{2}} \operatorname{rg}_{k}\left(M_{n, d}\right) X^{n} Y^{d} .
$$

On note $Q_{M}=P_{M} \prod_{i=1}^{m}\left(1-X^{f_{1}(i)} Y^{f_{2}(i)}\right)$.

La série de Poincaré est additive par rapport aux suites exactes courtes : si $0 \longrightarrow M^{\prime} \longrightarrow M \longrightarrow M^{\prime \prime} \longrightarrow 0$ est une suite exacte de $k[f]$-modules bigradués de type fini, alors $P_{M}=P_{M^{\prime}}+P_{M^{\prime \prime}}$.

La proposition suivante est un analogue d'un résultat classique des séries de Poincaré à une variable.

Proposition 3.1.1. - Avec les notations au-dessus, on a $Q_{M} \in \mathbb{Z}\left[X, Y, X^{-1}, Y^{-1}\right]$. 
Démonstration. - On raisonne par récurrence en $m$. Si $m=0$, alors $k[f]=k$. Comme $M$ est un $k$-module de type fini, $P_{M} \in \mathbb{Z}\left[X, Y, X^{-1}, Y^{-1}\right]$. On suppose que la proposition a été démontrée pour les modules bigradués sur une algèbre des polynômes à $m-1$ variables. Soit $f^{\prime}$ la restriction de $f$ à $\{1, \ldots, m-1\}$. On note $\left(n_{m}, d_{m}\right)=f(m)$. L'application $T_{m}: M\left(-n_{m},-d_{m}\right) \longrightarrow M$ est un homomorphisme de $k[f]$-modules bigradués. Soit $N$ son noyau (vu comme un sous- $k[f]$-module bigradué de $M$ ). La suite suivante est exacte :

$$
0 \longrightarrow N\left(-n_{m},-d_{m}\right) \longrightarrow M\left(-n_{m},-d_{m}\right) \stackrel{T_{m}}{\longrightarrow} M \longrightarrow M / T_{m} M \longrightarrow 0 .
$$

Par conséquent,

$$
P_{M}-X^{n_{m}} Y^{d_{m}} P_{M}=P_{M / T_{m} M}-X^{n_{m}} Y^{d_{m}} P_{N} .
$$

Comme $M / T_{m} M$ et $N$ sont des $k\left[f^{\prime}\right]=k[f] /\left(T_{m}\right)$-modules bigradués de type fini, d'après l'hypothèse de récurrence, on a

$$
Q_{M}=Q_{M / T_{m} M}-X^{n_{m}} Y^{d_{m}} Q_{N} \in \mathbb{Z}\left[X, Y, X^{-1}, Y^{-1}\right] .
$$

Remarque 3.1.2. - Dans le cas particulier où $f_{1} \equiv 1$, l'algèbre $k[f]$ munie de la première graduation est l'algèbre de polynôme usuellement graduée. On peut également considérer la première graduation de $M$ pour laquelle la $n^{\text {ième }}$ composante homogène de $M$ est $\bigoplus_{d \in \mathbb{Z}} M_{n, d}$. Avec cette graduation, $M$ est un module gradué de type fini sur l'algèbre de polynômes $k\left[T_{1}, \ldots, T_{m}\right]$ usuellement graduée. Si on désigne par $H_{M}$ la série de Poincaré classique de $M$ (pour la première graduation), alors on a la relation $H_{M}(X)=P_{M}(X, 1)$. La théorie classique des séries de Poincaré affirme qu'il existe $h \in\{0, \ldots, m\}$ tel que $H_{M}(X)$ s'écrive sous la forme

$$
H_{M}(X)=a_{h}(X)(1-X)^{-h}+a_{h-1}(X)(1-X)^{-h+1}+\cdots+a_{0}(X),
$$

où $a_{0}, \ldots, a_{h}$ sont des éléments dans $\mathbb{Z}\left[X, X^{-1}\right]$ et $a_{h}$ est à coefficients positifs, et est non-nul lorsque $M \neq 0$. D'autre part, les valeurs de $h$ et de $a_{h}(1)$ ne dépendent pas du choix de $\left(a_{0}, \ldots, a_{h}\right)$. Lorsque $M \neq 0$, la valeur de $h$ coïncide avec la dimension de $M$, c'est-à-dire la dimension de Krull de $k\left[T_{1}, \ldots, T_{m}\right] / \operatorname{ann}(M)$. On note $c(M)=a_{h}(1)$. Si $M=0$, on note par convention $\operatorname{dim}(M)=-\infty$ et $c(M)=0$.

Dans la suite, on présente un analogue de la formule (45) pour les séries de Poincaré à deux variables :

THÉORÈme 3.1.3. - Soient $f=\left(f_{1}, f_{2}\right):\{1, \ldots, m\} \rightarrow \mathbb{N}^{2}$ une application telle que $f_{1} \equiv 1$ et $M$ un $k[f]$-module bigradué de type fini. Alors la série $P_{M}$ peut s'écrire sous la forme

$$
P_{M}(X, Y)=\sum_{r=0}^{h} \sum_{\substack{\alpha \subset\{1, \ldots, m\} \\ \# \alpha=r}} I_{\alpha}(X, Y) \prod_{i \in \alpha}\left(1-X Y^{f_{2}(i)}\right)^{-1}
$$

où

1) $I_{\alpha} \in \mathbb{Z}\left[X, Y, X^{-1}, Y^{-1}\right]$, 
2) si $\# \alpha=h, I_{\alpha}$ est à coefficients positifs,

3) si $M \neq 0$, il existe au moins un $\alpha \subset\{1, \ldots, m\}$ de cardinal $h$ tel que $I_{\alpha} \neq 0$.

Pour simplifier la démonstration du théorème 3.1.3, on introduit la notation suivante : si $M$ est un $k[f]$-module bigradué de type fini satisfaisant à l'assertion du théorème 3.1 .3 , on dit que $M$ vérifie la condition $\mathbb{P}$, noté $\mathbb{P}(M)$. Avec cette notation, l'énoncé du théorème 3.1 .3 se simplifie comme :

pour tout $k[f]$-module bigradué de type fini $M$, on a $\mathbb{P}(M)$.

Pour tout entier $m>0$, soit $\Theta_{m}$ l'ensemble

$$
\left\{(i, j) \in \mathbb{Z}^{2} \mid 0 \leqslant i \leqslant m, j>0\right\} \cup\{(-\infty, 0)\} .
$$

On le munit de la relation lexicographique " $\leqslant$ " comme ci-dessous:

$$
(i, j) \leqslant\left(i^{\prime}, j^{\prime}\right) \text { si et seulement si } i<i^{\prime} \text { ou si } i=i^{\prime}, j \leqslant j^{\prime} .
$$

C'est une relation d'ordre. L'ensemble $\Theta_{m}$ est totalement ordonné pour cette relation. On utilisera l'expression " $(i, j)<\left(i^{\prime}, j^{\prime}\right)$ " pour désigner la condition

$$
(i, j) \leqslant\left(i^{\prime}, j^{\prime}\right) \quad \text { mais }(i, j) \neq\left(i^{\prime}, j^{\prime}\right) .
$$

LEMme 3.1.4. - Soit $0 \longrightarrow M^{\prime} \longrightarrow M \longrightarrow M^{\prime \prime} \longrightarrow 0$ une suite exacte courte de $k[f]$-modules bigradués de type fini. Alors

1) $\operatorname{dim} M=\max \left(\operatorname{dim} M^{\prime}, \operatorname{dim} M^{\prime \prime}\right)$,

2) $c(M)= \begin{cases}c\left(M^{\prime}\right)+c\left(M^{\prime \prime}\right), & \operatorname{dim} M^{\prime}=\operatorname{dim} M^{\prime \prime}, \\ c\left(M^{\prime}\right), & \operatorname{dim} M^{\prime}>\operatorname{dim} M^{\prime \prime}, \\ c\left(M^{\prime \prime}\right), & \operatorname{dim} M^{\prime \prime}>\operatorname{dim} M^{\prime} .\end{cases}$

3) $\mathbb{P}\left(M^{\prime}\right)$ et $\mathbb{P}\left(M^{\prime \prime}\right) \Longrightarrow \mathbb{P}(M)$.

Démonstration. - C'est une conséquence des égalités $P_{M}=P_{M^{\prime}}+P_{M^{\prime \prime}}$ et $H_{M}=$ $H_{M^{\prime}}+H_{M^{\prime \prime}}$.

Démonstration du théorème 3.1.3. - On raisonne par récurrence en $m$ en commençant par montrer que le théorème est vrai pour le cas où $\operatorname{dim} M \leqslant 0$. Si $M$ est de dimension $\leqslant 0$, alors la série de Poincaré classique de $M$, i.e., $H_{M}(X)=P_{M}(X, 1)$ est un élément de $\mathbb{Z}\left[X, X^{-1}\right]$, et on a $P_{M} \in \mathbb{Z}\left[X, Y, X^{-1}, Y^{-1}\right]$. Donc la condition $\mathbb{P}(M)$ est vérifiée. Comme $\operatorname{dim} M \leqslant m$, le théorème est vrai lorsque $m=0$. On suppose que le théorème est vrai pour les modules bigradués sur une algèbre des polynômes à au plus $m-1$ variables $(m \geqslant 1)$. Soient $f=\left(f_{1}, f_{2}\right):\{1, \ldots, m\} \rightarrow \mathbb{N}^{2}$ une application telle que $f_{1} \equiv 1$ et $M$ un $k[f] \cong k\left[T_{1}, \ldots, T_{m}\right]$-module bigradué de type fini. Soit $d=f_{2}(m)$. On commence un autre procédé de récurrence en $(\operatorname{dim} M, c(M))$. La condition $\mathbb{P}(M)$ a été déjà démontrée pour $\operatorname{dim} M \leqslant 0$. On suppose qu'elle a aussi été prouvée pour le cas où $(\operatorname{dim} M, c(M))<(r, s)$, où $0<r \leqslant m, s>0$. Dans la suite, on démontre $\mathbb{P}(M)$ pour le cas où $(\operatorname{dim} M, c(M))=(r, s)$. On considère l'homothétie $T_{m}: M(-1,-d) \longrightarrow M$, qui est un homomorphisme de $k[f]$-modules bigradués. On désigne par $f^{\prime}$ la restriction de $f$ à $\{1, \ldots, m-1\}$. Soit $N_{1}$ le noyau de $T_{m}$ (vu comme un sous- $k[f]$-module bigradué de $M)$. C'est un $k\left[f^{\prime}\right]$-module bigradué de type fini. D'après l'hypothèse 
de récurrence, $\mathbb{P}\left(N_{1}\right)$ est vraie. Soit $M_{1}=M / N_{1}$. D'après le lemme 3.1.4 3), pour démontrer $\mathbb{P}(M)$, il suffit de démontrer $\mathbb{P}\left(M_{1}\right)$. Si $\operatorname{dim} N_{1}=\operatorname{dim} M$, alors ou bien $\operatorname{dim} M_{1}<\operatorname{dim} M$, ou bien $\operatorname{dim} M_{1}=\operatorname{dim} M$ et $c\left(M_{1}\right)=c(M)-c\left(N_{1}\right)<c(M)$. Donc on a toujours $\left(\operatorname{dim} M_{1}, c\left(M_{1}\right)\right)<(\operatorname{dim} M, c(M))$. D'après l'hypothèse de récurrence on obtient $\mathbb{P}\left(M_{1}\right)$. Sinon, on a $\operatorname{dim} N_{1}<\operatorname{dim} M$ et $\left(\operatorname{dim} M_{1}, c\left(M_{1}\right)\right)=(\operatorname{dim} M, c(M))$. Si $\mathbb{P}(M)$ n'était pas vrai, en itérant le procédé comme ci-dessus on obtiendrait une suite croissante de sous-modules homogènes

$$
N_{1} \subset N_{2} \subset \cdots N_{j} \subset N_{j+1} \subset \cdots
$$

de $M$ telle que,

i) $N_{j}=\operatorname{Ker} T_{m}^{j}$,

ii) $\operatorname{dim} N_{j}<\operatorname{dim} M$,

iii) $M_{j}:=M / N_{j}$ ne satisfait pas à la condition $\mathbb{P}$, et $\left(\operatorname{dim} M_{j}, c\left(M_{j}\right)\right)=$ $(\operatorname{dim} M, c(M))$.

Comme $k[f]$ est un anneau noethérien, la suite (47) est stationnaire. Autrement dit, il existe $j \in \mathbb{N}$ tel que $N_{j}=N_{j+1}$. Cela montre que l'application d'homothétie $T_{m}$ : $M_{j}(-1,-d) \rightarrow M_{j}$ est injective. On considère la suite exacte

$$
0 \longrightarrow M_{j}(-1,-d) \stackrel{T_{m}}{\longrightarrow} M_{j} \longrightarrow M_{j} / T_{m} M_{j} \longrightarrow 0 .
$$

Soit $N^{\prime}=M_{j} / T_{m} M_{j}$. C'est en fait un $k\left[f^{\prime}\right]$-module de type fini. D'après l'hypothèse de récurrence on a $\mathbb{P}\left(N^{\prime}\right)$. Enfin, comme $\left(1-X Y^{d}\right) P_{M_{j}}(X, Y)=P_{N^{\prime}}(X, Y)$, on obtient $\mathbb{P}\left(M_{j}\right)$. Cela est absurde. Donc la condition $\mathbb{P}(M)$ est vérifiée.

3.1.2. Mesures associées à une série à deux variables. - On associe à chaque série formelle dans $\mathbb{Z} \llbracket X, Y \rrbracket\left[X^{-1}, Y^{-1}\right]$ dont les coefficients sont positifs une suite de mesures boréliennes sur $\mathbb{R}$ et ensuite étudie le comportement asymptotique de ces mesures. On verra plus loin que, si la série formelle que l'on considère est la série de Poincaré d'un module bigradué, alors ces mesures coïncident avec les mesures associées aux filtrations induites par la deuxième graduation.

Soit $P$ une série formelle dans $\mathbb{Z} \llbracket X, Y \rrbracket\left[X^{-1}, Y^{-1}\right]$ à coefficients positifs. Alors $P$ s'écrit sous la forme $P(X, Y)=\sum_{(n, d) \in \mathbb{Z}^{2}} a_{n, d}(P) X^{n} Y^{d}$. Pour tout $n \in \mathbb{N}$, on note $S_{n}(P)=\sum_{d \in \mathbb{Z}} a_{n, d}(P)$. On désigne par $\nu_{n, P}$ la mesure borélienne sur $\mathbb{R}$ de la forme

$$
\nu_{n, P}=\sum_{d \in \mathbb{Z}} \frac{a_{n, d}(P)}{S_{n}(P)} \delta_{d / n} .
$$

Si $S_{n}(P)=0$, alors par convention $\nu_{n, P}$ est la mesure nulle.

On s'intéresse à étudier la convergence vague des mesures $\nu_{n, P}$ pour une série de Poincaré à deux variables, c'est-à-dire une série $P$ de la forme (46). Une telle série sous la forme la plus simple est celle dans la proposition au-dessous. Dans ce cas-là la suite des mesures $\nu_{n, P}$ converge vaguement vers une mesure borélienne, qui est 
l'image directe de la mesure de Lebesgue sur un simplexe par une projection linéaire. On montrera dans le théorème 3.1.7 que la convergence au cas général se déduit naturellement $\mathrm{du}$ résultat dans ce cas particulier, et la limite des mesures est une combinaison linéaire d'images directes de mesures de Lebesgue.

Pour tout entier $m \geqslant 1$, soit $\Delta_{m}$ le simplexe

$$
\Delta_{m}=\left\{\left(y_{1}, \ldots, y_{m}\right) \in \mathbb{R}_{+}^{m} \mid y_{1}+\cdots+y_{m}=1\right\} .
$$

Si $\underline{u}=\left(u_{1}, \ldots, u_{m}\right)$ est un élément dans $\mathbb{R}^{m}$, on désigne par $\varphi_{\underline{u}}: \Delta_{m} \rightarrow \mathbb{R}$ l'application qui envoie $\left(y_{1}, \ldots, y_{m}\right)$ en $y_{1} u_{1}+\cdots+y_{m} u_{m}$ et par $\nu_{\underline{u}}$ l'image directe de la mesure de Lebesgue sur $\Delta_{m}$ (normalisée de sorte que la masse totale est 1) par l'application $\varphi_{\underline{u}}$. C'est une mesure de probabilité. Enfin, soit $\nu_{\varnothing}$ la mesure nulle par convention.

On fixe dans la suite du sous-paragraphe un entier $m \geqslant 1$ et un élément $\underline{u}=$ $\left(u_{1}, \ldots, u_{m}\right) \in \mathbb{N}^{m}$.

Proposition 3.1.5. - Si P est une série dans $\mathbb{Z} \llbracket X, Y \rrbracket$ de la forme

$$
P(X, Y)=\prod_{i=1}^{m}\left(1-X Y^{u_{i}}\right)^{-1},
$$

alors les mesures boréliennes $\nu_{n, P}$ convergent vaguement vers $\nu_{\underline{u}}$ lorsque $n \rightarrow+\infty$.

Démonstration. - On a

$$
\begin{aligned}
& P(X, Y)=\prod_{i=1}^{m} \sum_{n \geqslant 0} X^{n} Y^{n u_{i}}=\sum_{\substack{(n, d) \in \mathbb{Z}^{2} \\
n>0}} X^{n} Y^{d} \sum_{\substack{(n, d) \in \mathbb{N} \times \mathbb{Z} \\
\left(\begin{array}{c}
\left.y_{1}, \ldots, y_{m}\right) \in \frac{1}{n} \mathbb{N}^{m} \\
y_{1}+\cdots+y_{m}
\end{array}=1, y_{1} u_{1}+\cdots+y_{m} u_{m}=d / n\right.}} 1 . \\
&=1+\sum_{\begin{array}{c}
\left(a_{1}, \ldots, a_{m}\right) \in \mathbb{N}^{m} \\
a_{1}+\cdots+a_{m}=n, \\
a_{1} u_{1}+\cdots+a_{m} u_{m}=d
\end{array}} 1
\end{aligned}
$$

En outre,

$$
S_{n}(P)=\sum_{\substack{\left(y_{1}, \ldots, y_{m}\right) \in \frac{1}{n} \mathbb{N}^{m} \\ y_{1}+\cdots+y_{m}=1}} 1 .
$$

Pour tout entier $n>0$, soit $\eta_{n, P}$ la mesure sur $\Delta_{m}$ définie par

$$
\eta_{n, P}=\sum_{\underline{y} \in \frac{1}{n} \mathbb{N}^{m} \cap \Delta_{m}} \frac{1}{S_{n}(P)} \delta_{\underline{y}} .
$$

On observe que $\nu_{n, P}$ est l'image directe de $\eta_{n, P}$ par $\varphi_{\underline{u}}$. Par conséquent, $\nu_{n, P}$ est à support dans $\varphi_{\underline{u}}\left(\Delta_{m}\right)$. Donc pour toute fonction continue $f: \mathbb{R} \rightarrow \mathbb{R}, f$ est intégrable par rapport à la mesure $\nu_{n, P}$. Par la formule du changement de variables, on a

$$
\int_{\mathbb{R}} f \mathrm{~d} \nu_{n, P}=\int_{\Delta_{m}} f \circ \varphi_{\underline{u}} \mathrm{~d} \eta_{n, P},
$$


qui est la $n^{\text {ième }}$ somme de Riemann de la fonction $f \circ \varphi_{\underline{u}}: \Delta_{m} \rightarrow \mathbb{R}$. Donc la suite $\left(\int_{\mathbb{R}} f \mathrm{~d} \nu_{n, P}\right)_{n \geqslant 1}$ converge vers $\int_{\Delta_{m}} f \circ \varphi_{\underline{u}} \mathrm{~d} \eta=\int_{\mathbb{R}} f \mathrm{~d} \varphi_{\underline{u} *} \eta$, où $\eta$ est la mesure de Lebesgue sur $\Delta_{m}$. On en déduit alors que les mesures $\nu_{n, P}$ convergent vaguement vers $\varphi_{\underline{u} *} \eta$.

Corollaire 3.1.6. - Si $Q$ est une série non-nulle dans $\mathbb{Z}\left[X, Y, X^{-1}, Y^{-1}\right]$ à coefficients positifs, et si $P \in \mathbb{Z} \llbracket X, Y \rrbracket\left[X^{-1}, Y^{-1}\right]$ est de la forme

$$
P(X, Y)=Q(X, Y) \prod_{i=1}^{m}\left(1-X Y^{u_{i}}\right)^{-1}
$$

alors les mesures boréliennes $\nu_{n, P}$ convergent vaguement vers $\nu_{\underline{u}}$ lorsque $n \rightarrow+\infty$.

Démonstration. - Soit $P^{\prime}=\prod_{i=1}^{m}\left(1-X Y^{u_{i}}\right)^{-1}$. On suppose que $Q$ soit de la forme

$$
Q(X, Y)=\sum_{\left|n^{\prime}\right| \leqslant b\left|d^{\prime}\right| \leqslant r} c_{n^{\prime}, d^{\prime}} X^{n^{\prime}} Y^{d^{\prime}}
$$

où $c_{n^{\prime}, d^{\prime}} \geqslant 0$. Comme $P=P^{\prime} Q$,

$$
a_{n, d}(P)=\sum_{\left|n^{\prime}\right| \leqslant b} \sum_{\left|d^{\prime}\right| \leqslant r} c_{n^{\prime}, d^{\prime}} a_{n-n^{\prime}, d-d^{\prime}}\left(P^{\prime}\right)
$$

et

$$
\begin{aligned}
S_{n}(P) & =\sum_{d \in \mathbb{Z}} a_{n, d}(P)=\sum_{d \in \mathbb{Z}} \sum_{\left|n^{\prime}\right| \leqslant b\left|d^{\prime}\right| \leqslant r} \sum_{n^{\prime}, d^{\prime}} a_{n-n^{\prime}, d-d^{\prime}}\left(P^{\prime}\right) \\
& =\sum_{\left|n^{\prime}\right| \leqslant b} \sum_{\left|d^{\prime}\right| \leqslant r} \sum_{d \in \mathbb{Z}} c_{n^{\prime}, d^{\prime}} a_{n-n^{\prime}, d-d^{\prime}}\left(P^{\prime}\right)=\sum_{\left|n^{\prime}\right| \leqslant b\left|d^{\prime}\right| \leqslant r} \sum_{n^{\prime}, d^{\prime}} S_{n-n^{\prime}}\left(P^{\prime}\right) .
\end{aligned}
$$

On note $C_{n^{\prime}}=\sum_{\left|d^{\prime}\right| \leqslant r} c_{n^{\prime}, d^{\prime}}$. Avec cette notation, on a $S_{n}(P)=\sum_{\left|n^{\prime}\right| \leqslant b} C_{n^{\prime}} S_{n-n^{\prime}}\left(P^{\prime}\right)$. Si $g: \mathbb{R} \rightarrow \mathbb{R}$ est une fonction continue à support compact, alors

$$
\int_{\mathbb{R}} g \mathrm{~d} \nu_{n, P}=\sum_{d \in \mathbb{Z}} \frac{a_{n, d}(P)}{S_{n}(P)} g(d / n)=\frac{1}{S_{n}(P)} \sum_{d \in \mathbb{Z}} \sum_{\left|n^{\prime}\right| \leqslant b\left|d^{\prime}\right| \leqslant r} \sum_{n^{\prime}, d^{\prime}} a_{n-n^{\prime}, d-d^{\prime}}\left(P^{\prime}\right) g(d / n) .
$$

La suite des nombres de la forme

$$
\begin{aligned}
& \frac{1}{S_{n}(P)} \sum_{\left|n^{\prime}\right| \leqslant b} \sum_{\left|d^{\prime}\right| \leqslant r} \sum_{d \in \mathbb{Z}} c_{n^{\prime}, d^{\prime}} a_{n-n^{\prime}, d-d^{\prime}}\left(P^{\prime}\right) g\left(\frac{d-d^{\prime}}{n-n^{\prime}}\right) \\
= & \frac{1}{S_{n}(P)} \sum_{\left|n^{\prime}\right| \leqslant b} \sum_{\left|d^{\prime}\right| \leqslant r} c_{n^{\prime}, d^{\prime}} S_{n-n^{\prime}}\left(P^{\prime}\right) \int_{\mathbb{R}} g \mathrm{~d} \nu_{n-n^{\prime}, P^{\prime}} \\
= & \frac{1}{S_{n}(P)} \sum_{\left|n^{\prime}\right| \leqslant b} C_{n^{\prime}} S_{n-n^{\prime}}\left(P^{\prime}\right) \int_{\mathbb{R}} g \mathrm{~d} \nu_{n-n^{\prime}, P^{\prime}}
\end{aligned}
$$


converge vers $\int_{\mathbb{R}} g \mathrm{~d} \nu_{\underline{u}}$ puisque les mesures $\nu_{n, P^{\prime}}$ convergent vaguement vers $\nu_{\underline{u}}$ lorsque $n$ tend vers l'infini. La fonction $g$ étant uniformément continue sur $\mathbb{R}$, pour tout $\delta>0$, il existe $\varepsilon>0$ tel que, pour tous $x, y \in \mathbb{R}$ vérifiant $|x-y|<\varepsilon$, on ait $|g(x)-g(y)|<\delta$. Soit $d_{0}=\max _{1 \leqslant i \leqslant m}\left|u_{i}\right|$. Comme $P^{\prime}=\prod_{i=1}^{m}\left(1-X Y^{u_{i}}\right)^{-1}$, si $|d|>d_{0} n$, alors $a_{n, d}\left(P^{\prime}\right)=0$. Par conséquent, pour tous entiers $n, d, n^{\prime}$ et $d^{\prime}$ tels que $n>b,\left|n^{\prime}\right| \leqslant b,\left|d^{\prime}\right| \leqslant r$ et que $a_{n-n^{\prime}, d-d^{\prime}}\left(P^{\prime}\right) \neq 0$, on a

$$
\left|\frac{d}{n}-\frac{d-d^{\prime}}{n-n^{\prime}}\right|=\left|\frac{d^{\prime} n-d n^{\prime}}{n\left(n-n^{\prime}\right)}\right| \leqslant \frac{r}{n-n^{\prime}}+\frac{b\left((n+b) d_{0}+r\right)}{n\left(n-n^{\prime}\right)} .
$$

Donc il existe un entier $N>0$ tel que, pour tous entiers $n, d, n^{\prime}$ et $d^{\prime}$ vérifiant $n \geqslant N$, $\left|n^{\prime}\right| \leqslant b$ et $\left|d^{\prime}\right| \leqslant r$, on ait, ou bien $a_{n-n^{\prime}, d-d^{\prime}}\left(P^{\prime}\right)=0$, ou bien $\left|\frac{d}{n}-\frac{d-d^{\prime}}{n-n^{\prime}}\right|<\varepsilon$. On obtient donc

$$
\begin{aligned}
& \left|\int_{\mathbb{R}} g \mathrm{~d} \nu_{n, P}-\frac{1}{S_{n}(P)} \sum_{\left|n^{\prime}\right| \leqslant b} \sum_{\left|d^{\prime}\right| \leqslant r} \sum_{d \in \mathbb{Z}} c_{n^{\prime}, d^{\prime}} a_{n-n^{\prime}, d-d^{\prime}}\left(P^{\prime}\right) g\left(\frac{d-d^{\prime}}{n-n^{\prime}}\right)\right| \\
& \leqslant \frac{1}{S_{n}(P)} \sum_{\left|n^{\prime}\right| \leqslant b} \sum_{\left|d^{\prime}\right| \leqslant r} \sum_{d \in \mathbb{Z}} c_{n^{\prime}, d^{\prime}} a_{n-n^{\prime}, d-d^{\prime}}\left(P^{\prime}\right)\left|g\left(\frac{d}{n}\right)-g\left(\frac{d-d^{\prime}}{n-n^{\prime}}\right)\right| \\
& \leqslant \frac{\delta}{S_{n}(P)} \sum_{\left|n^{\prime}\right| \leqslant b} \sum_{\left|d^{\prime}\right| \leqslant r} \sum_{d \in \mathbb{Z}} c_{n^{\prime}, d^{\prime}} a_{n-n^{\prime}, d-d^{\prime}}\left(P^{\prime}\right)=\delta .
\end{aligned}
$$

On en déduit la convergence vague des $\nu_{n, P}$ vers $\nu_{\underline{u}}$.

ThÉORÈme 3.1.7. - Soit

$$
P(X, Y)=\sum_{r=0}^{h} \sum_{\substack{\alpha \subset\{1, \ldots, m\} \\ \# \alpha=r}} I_{\alpha}(X, Y) \prod_{i \in \alpha}\left(1-X Y^{u_{i}}\right)^{-1}
$$

une série dans $\mathbb{Z} \llbracket X, Y \rrbracket\left[X^{-1}, Y^{-1}\right]$ où

a) $P$ est à coefficients positifs,

b) $I_{\alpha} \in \mathbb{Z}\left[X, Y, X^{-1}, Y^{-1}\right]$,

c) pour tout $\alpha \subset\{1, \ldots, m\}$ de cardinal $h, I_{\alpha}$ est à coefficients positifs,

d) il existe au moins un $\alpha \subset\{1, \ldots, m\}$ de cardinal $h$ tel que $I_{\alpha} \neq 0$.

Alors les mesures boréliennes $\nu_{n, P}$ convergent vaguement vers une mesure borélienne $\nu_{P}$ lorsque $n \rightarrow+\infty$. De plus, si pour tout $\alpha=\left\{i_{1}<\cdots<i_{h}\right\}$ on note $\underline{u}_{\alpha}=$ $\left(u_{i_{1}}, \ldots, u_{i_{h}}\right)$, alors la mesure limite $\nu_{P}$ est égale $\grave{a}$

$$
\sum_{\substack{\alpha \subset\{1, \ldots, m\} \\ \# \alpha=h}} \frac{I_{\alpha}(1,1)}{S} \nu_{\underline{u}_{\alpha}} \quad \text { ò̀ } \quad S=\sum_{\substack{\alpha \subset\{1, \ldots, m\} \\ \# \alpha=h}} I_{\alpha}(1,1) .
$$

Donc $\nu_{P}$ est une mesure de probabilité lorsque $h>0$. Si $h=0$, alors $\nu_{P}$ est la mesure nulle. 
Démonstration. - Pour toute série $Q \in \mathbb{Z} \llbracket X, Y \rrbracket\left[X^{-1}, Y^{-1}\right]$, on utilise l'expression $a_{n, d}(Q)$ pour désigner le coefficient du terme $X^{n} Y^{d}$ dans $Q$. Pour tout $n \in \mathbb{Z}$, si $a_{n, d}(Q)$ est nulle pour tout sauf un nombre fini de $d$, alors on note $S_{n}(Q)$ la somme $\operatorname{des} a_{n, d}(Q)$. Soit $\mathscr{A}$ le sous-ensemble de $\mathbb{Z} \llbracket X, Y \rrbracket\left[X^{-1}, Y^{-1}\right]$ des séries $Q$ telles que, pour tout $n \in \mathbb{Z}, a_{n, d}(Q)$ est nulle pour tout sauf un nombre fini de $d$. C'est en fait un sous-groupe additif de $\mathbb{Z} \llbracket X, Y \rrbracket\left[X^{-1}, Y^{-1}\right]$. De plus, l'application $S_{n}: \mathscr{A} \rightarrow \mathbb{Z}$ est un homomorphisme de groupe. De plus, pour tout $Q \in \mathscr{A}$, la série $Q(X, 1)$ est bien définie. En particulier, si $Q$ est la série de la forme

$$
Q(X, Y)=I(X, Y) \prod_{i \in \alpha}\left(1-X Y^{u_{i}}\right)^{-1}, \quad \text { où } \alpha \subset\{1, \ldots, m\}, I \in \mathbb{Z}\left[X, Y, X^{-1}, Y^{-1}\right],
$$

alors $Q \in \mathscr{A}$. En outre, on a

$$
Q(X, 1)=I(X, 1)(1-X)^{-\# \alpha}
$$

et donc

$$
S_{n}(Q)=\frac{I(1,1)}{(\# \alpha-1) !} n^{\# \alpha-1}+o\left(n^{\# \alpha-1}\right) \quad(n \rightarrow \infty) .
$$

Pour tout $\alpha \subset\{1, \ldots, m\}$ tel que $\# \alpha=h$, on note

$$
P_{\alpha}^{(1)}(X, Y)=I_{\alpha}(X, Y) \prod_{i \in \alpha}\left(1-X Y^{u_{i}}\right)^{-1} .
$$

Soient

$$
P^{(1)}=\sum_{\substack{\alpha \subset\{1, \ldots, m\} \\ \# \alpha=h}} P_{\alpha}^{(1)} \text { et } P^{(2)}=P-P^{(1)} .
$$

D'après le corollaire 3.1.6, les mesures $\nu_{n, P_{\alpha}^{(1)}}$ convergent vaguement vers $\nu_{\underline{u}_{\alpha}}$ lorsque $I_{\alpha} \neq 0$, et convergent vaguement vers la mesure nulle lorsque $I_{\alpha}=0$. De plus, on a

$$
\nu_{n, P^{(1)}}=\sum_{\substack{\alpha \subset\{1, \ldots, m\} \\ \# \alpha=h}} \frac{S_{n}\left(P_{\alpha}^{(1)}\right)}{S_{n}\left(P^{(1)}\right)} \nu_{n, P_{\alpha}^{(1)}} .
$$

En outre, l'additivité de la fonction $S_{n}$ montre que $\lim _{n \rightarrow \infty} \frac{S_{n}\left(P_{\alpha}^{(1)}\right)}{S_{n}\left(P^{(1)}\right)}=\frac{I_{\alpha}(1,1)}{S}$. On obtient donc que les mesures $\nu_{n, P^{(1)}}$ convergent vaguement vers $\sum_{\substack{\alpha \subset\{1, \ldots, m\} \\ \# \alpha=h}} \frac{I_{\alpha}(1,1)}{S} \nu_{u_{\alpha}}$.

Enfin, comme les $I_{\alpha}(\# \alpha=h)$ sont à coefficients positifs et comme l'un parmi eux est non-nul, on obtient $\lim _{n \rightarrow+\infty} S_{n}\left(P^{(2)}\right) / S_{n}\left(P^{(1)}\right)=0$ car $S_{n}\left(P^{(2)}\right)=o\left(n^{h-1}\right)$ et $S_{n}\left(P^{(1)}\right) \gg n^{h-1}$. On en déduit donc que les mesures

$$
\nu_{n, P}=\frac{1}{S_{n}(P)}\left(S_{n}\left(P^{(1)}\right) \nu_{n, P^{(1)}}+\sum_{d \in \mathbb{Z}} a_{n, d}\left(P^{(2)}\right) \delta_{d / n}\right)
$$

convergent vaguement vers la limite $\operatorname{des} \nu_{n, P^{(1)}}$. 
3.1.3. Convergence des polygones. - On s'intéresse maintenant à la convergence des polygones associés à un module bigradué. Soient $m \geqslant 1$ un entier et $\underline{u}=$ $\left(u_{1}, \ldots, u_{m}\right) \in \mathbb{N}^{m}$. Soit $f=\left(f_{1}, f_{2}\right):\{1, \ldots, m\} \rightarrow \mathbb{N}^{2}$ une application telle que $f_{1} \equiv 1$ et que $f_{2}(i)=u_{i}$. Soient $A=k[f]$ et $M$ un $A$-module bigradué de type fini.

Soit $P=P_{M}$ la série de Poincaré à deux variables de $M$. Pour tout entier $n \geqslant 1$, on munit l'espace vectoriel $M_{n, \bullet}:=\bigoplus_{d \in \mathbb{Z}} M_{n, d}$ de la $\mathbb{R}$-filtration $\mathcal{F}^{(n)}$ définie par $\mathcal{F}_{\lambda}^{(n)} M_{n, \bullet}=\bigoplus_{d \geqslant \lambda} M_{n, d}$, alors la mesure $\nu_{n, P}$ définie dans (48) s'identifie à $T_{\frac{1}{n}} \nu_{\mathcal{F}^{(n)}}$, où $\nu_{\mathcal{F}^{(n)}}$ est la mesure associée à la filtration $\mathcal{F}^{(n)}$ définie dans $\S 1.2 .2$, et $T_{\frac{1}{n}}$ est l'opération de dilatation définie dans $§ 1.2 .4$. On suppose que $M_{n, \bullet}$ est non-nul lorsque $n$ est assez grand et donc $\nu_{\mathcal{G}^{(n)}}$ est une mesure de probabilité. Le théorème 3.1.7 combiné avec la proposition 1.2.9, implique le résultat suivant :

THÉORÈmE 3.1.8. - La suite des polygones de la forme $\frac{1}{n} \mathscr{P}\left(\nu_{\mathcal{F}^{(n)}}\right)$ converge uniformément vers une fonction concave sur $[0,1]$ lorsque $n$ tend vers l'infini. De plus, en écrivant la série $P$ sous la forme (49) (cela est toujours possible d'après le théorème 3.1.3), la limite des polygones coïncide avec $\mathscr{P}\left(\nu_{P}\right)$, où $\nu_{P}$ est la mesure de probabilité définie dans (50).

Démonstration. - D'après le théorème 3.1 .7 , la suite de mesures $\left(T_{\frac{1}{n}} \nu_{\mathscr{F}^{(n)}}\right)_{n \geqslant 1}$ converge vaguement vers $\nu_{P}$. D'après $(6)$, on a $\mathscr{P}\left(T_{\frac{1}{n}} \nu_{\mathscr{G}^{(n)}}\right)=\frac{1}{n} \mathscr{P}\left(\nu_{\mathscr{F}^{(n)}}\right)$. La proposition 1.2.9 montre alors que $\left(\frac{1}{n} \mathscr{P}\left(\nu_{\mathscr{F}^{(n)}}\right)\right)_{n \geqslant 1}$ converge uniformément vers $\mathscr{P}\left(\nu_{P}\right)$.

EXEMPle 3.1.9. - On considère un cas particulier où $M=A$. Dans ce cas-là, la limite $\nu_{P}$ des mesures est $\nu_{\underline{u}}$ - l'image directe de la mesure de Lebesgue sur $\Delta_{m}$ par l'application linéaire $\varphi_{\underline{u}}$, compte tenu de la proposition 3.1.5. Par conséquent, la fonction $F_{\nu_{u}}(x)=\nu_{\underline{u}}(] x,+\infty[)$ est continue. Donc il en est de même de la fonction $F_{\nu_{\underline{u}}}^{*}$ (voir $\S 1.2 .5$ pour la définition). On en déduit que la limite des polygone $\mathscr{P}\left(\nu_{\underline{u}}\right)$ est de classe $C^{1}$. Si de plus $n=2$, alors $\Delta_{2}=\{(x, 1-x) \mid x \in[0,1]\}$ est paramétré par $[0,1]$, et l'application $\varphi_{\underline{u}}: \Delta_{2} \rightarrow \mathbb{R}$ envoie $(x, 1-x)$ en $u_{1} x+u_{2}(1-x)$. Donc $\nu_{\underline{u}}$ est la mesure équiprobable sur l'intervalle délimité par $u_{1}$ et $u_{2}$. La fonction $F_{\nu_{\underline{u}}}(x)$ est égale à $\left|\frac{\left(u_{2}-x\right)_{+}-\left(u_{1}-x\right)_{+}}{u_{2}-u_{1}}\right|$ si $u_{1} \neq u_{2}$ et à $\mathbb{1}_{] u_{1},+\infty[}(x)$ si $u_{1}=u_{2}$. On en déduit $F_{\nu_{\underline{u}}}^{*}(t)=-\left|u_{1}-u_{2}\right| t+\max \left(u_{1}, u_{2}\right)$ et donc $\mathscr{P}\left(\nu_{\underline{u}}\right)(t)=-\frac{1}{2}\left|u_{1}-u_{2}\right| t^{2}+\max \left(u_{1}, u_{2}\right) t$.

Remarque 3.1.10. - Dans certains cas particuliers on peut généraliser le théorème 3.1.8. Soit $B=k\left[X_{1}, \ldots, X_{m}\right]$ l'algèbre des polynômes, munie de la graduation usuelle. Pour tout $\alpha=\left(\alpha_{1}, \ldots, \alpha_{m}\right) \in \mathbb{N}^{m}$, on utilise le symbole $X^{\alpha}$ pour désigner le monôme $X_{1}^{\alpha_{1}} \cdots X_{m}^{\alpha_{m}}$ et on note $|\alpha|=\alpha_{1}+\cdots+\alpha_{m}$. On suppose que, pour tout entier $n \geqslant 1$, l'espace $B_{n}$ des polynômes homogènes de degré $n$ est muni d'une $\mathbb{R}$-filtration $\mathcal{F}^{(n)}$ telle que la base formée des monômes soit compatible à la filtration $\mathcal{F}^{(n)}$ et que 
$\lambda_{\mathscr{F}(|\alpha|+|\beta|)}\left(X^{\alpha+\beta}\right)=\lambda_{\mathscr{F}(|\alpha|)}\left(X^{\alpha}\right)+\lambda_{\mathscr{F}(|\beta|)}\left(X^{\beta}\right)$. Autrement dit, l'algèbre $B$ est " $\mathbb{N} \times \mathbb{R}$ bigraduée". Pour tout $i \in\{1, \ldots, m\}$ soit $b_{i}=\lambda_{\mathcal{F}^{(1)}}\left(X_{i}\right)$. Soit $\underline{b}=\left(b_{1}, \ldots, b_{m}\right)$. Par définition

$$
\nu_{\mathcal{F}^{(n)}}=\frac{1}{\operatorname{rg} B_{n}} \sum_{|\alpha|=n} \delta_{\alpha_{1} b_{1}+\cdots+\alpha_{m} b_{m}}, \quad T_{\frac{1}{n}} \nu_{\mathcal{F}^{(n)}}=\frac{1}{\operatorname{rg} B_{n}} \sum_{\underline{y} \in \frac{1}{n} \mathbb{N}^{m} \cap \Delta_{m}} \varphi_{\underline{b} *}\left(\delta_{\underline{y}}\right) .
$$

On en déduit donc que la suite de mesures $\left(T_{\frac{1}{n}} \nu_{\mathcal{F}^{(n)}}\right)_{n \geqslant 1}$ converge vaguement vers $\nu_{\underline{b}}$.

\subsection{Algèbres graduées quasi-filtrées et pseudo-filtrées}

Dans la section précédente, en utilisant la méthode des séries de Poincaré on a pu établir un théorème de convergence (le théorème 3.1.8) pour les algèbres bigraduées. En fait, le même résultat est aussi vrai pour une algèbre graduée munie des filtrations dont l'algèbre bigraduée associée est de type fini.

Soit $B=\bigoplus_{n \geqslant 0} B_{n}$ une algèbre graduée de type fini sur $k$. On suppose que chaque espace vectoriel $B_{n}$ est muni d'une $\mathbb{R}$-filtration décroissante $\mathcal{F}^{(n)}$ telle que le support de $\nu_{\mathscr{F}^{(n)}}$ soit contenu dans $\mathbb{N}$ (c'est-à-dire que les points de saut sont des entiers positifs) et que l'algèbre $B$ soit filtrée pour les filtrations $\mathcal{F}^{(n)}$, c'est-à-dire que $\mathcal{F}_{s}^{(n)} B_{n} \mathcal{F}_{t}^{\left(n^{\prime}\right)} B_{n^{\prime}} \subset \mathcal{F}_{s+t}^{\left(n+n^{\prime}\right)} B_{n+n^{\prime}}$ quels que soient $\left(n, n^{\prime}\right) \in \mathbb{N}^{2}$ et $(s, t) \in \mathbb{R}^{2}$. Pour tout entier $d$, soit $\widetilde{B}_{n, d}$ le sous-quotient $\mathcal{F}_{d}^{(n)} B_{n} / \mathcal{F}_{d+1}^{(n)} B_{n}$. Alors la somme directe $\widetilde{B}=\bigoplus_{n, d} \widetilde{B}_{n, d}$ forme une algèbre bigraduée sur $k$, où le produit de deux éléments $[x] \in B_{n, d}$ et $[y] \in B_{n^{\prime}, d^{\prime}}$ est la classe de $x y$ dans $\mathcal{F}_{d+d^{\prime}}^{\left(n+n^{\prime}\right)} B_{n+n^{\prime}} / \mathcal{F}_{d+d^{\prime}+1}^{\left(n+n^{\prime}\right)} B_{n+n^{\prime}}$. Soit $P \in \mathbb{Z} \llbracket X, Y \rrbracket$ la série

$$
P(X, Y):=\sum_{(n, d) \in \mathbb{N}^{2}} \operatorname{rg}_{k}\left(\widetilde{B}_{n, d}\right) X^{n} Y^{d}
$$

Il s'avère que la mesure dilatée $T_{\frac{1}{n}} \nu_{\mathcal{F}^{(n)}}$ associée à la filtration $\mathcal{F}^{(n)}$ coïncide avec $\nu_{n, P}$. Dans le cas où $\widetilde{B}$ est une algèbre de type fini sur $k$, cette observation nous permet d'appliquer le théorème 3.1 .7 pour démontrer la convergence $\operatorname{des} T_{\frac{1}{n}} \nu_{\mathcal{F}^{(n)}}$ et pour calculer la limite.

Cependant, il est très rare que la nouvelle algèbre $\widetilde{B}$ soit de type fini. On peut considérer un exemple simple où $B=k[X]$ est l'algèbre des polynômes à une variable. Soit $\left(a_{n}\right)_{n \geqslant 0}$ une suite d'entiers dans $\mathbb{N}$ telle que

$$
a_{0}=0, \quad 0 \leqslant a_{n} \leqslant n, \quad a_{n+n^{\prime}}>a_{n}+a_{n^{\prime}} \text { quel que soit }\left(n, n^{\prime}\right) \in \mathbb{Z}_{>0}^{2} .
$$

On suppose que $B$ est usuellement graduée et que la filtration $\mathcal{F}^{(n)}$ de l'espace $k X^{n}$ admet un seul point de saut en $a_{n}$. L'algèbre $B$ est filtrée car

$$
\lambda_{\mathscr{F}^{\left(n+n^{\prime}\right)}}\left(X^{n+n^{\prime}}\right)=a_{n+n^{\prime}} \geqslant a_{n}+a_{n^{\prime}}=\lambda_{\mathscr{F}^{(n)}}\left(X^{n}\right)+\lambda_{\mathscr{F}^{\left(n^{\prime}\right)}}\left(X^{n^{\prime}}\right),
$$

où les fonctions $\lambda$ sont définies dans (2). L'algèbre bigraduée $\widetilde{B}$ s'identifie à $k\left[T_{1}, \ldots, T_{n}, \cdots\right]$, où le bidegré de $T_{n}$ est $\left(n, a_{n}\right)$, modulo l'idéal homogène engendré 
par les éléments de la forme $T_{n} T_{n^{\prime}}$. Ce n'est pas une algèbre de type fini. Par ailleurs, la mesure associée à $\mathcal{F}^{(n)}$ est la mesure de Dirac $\delta_{a_{n}}$. La condition (51) combinée avec le corollaire 1.3.3 montre que la suite $\left(a_{n} / n\right)_{n \geqslant 1}$ converge dans $\mathbb{R}$. Cela montre effectivement que les mesures $T_{\frac{1}{n}} \delta_{a_{n}}=\delta_{a_{n} / n}$ convergent vaguement vers une mesure de Dirac lorsque $n$ tend vers l'infini.

Cet exemple suggère que la convergence vague des mesures associées à une algèbre graduée munie des filtrations devrait vérifier sous une hypothèse assez faible sur la croissance des filtrations. La formalisation de cette observation conduit à deux notions similaires - algèbre graduée quasi-filtrée et algèbre graduée pseudo-filtrée - qui sont importantes dans la deuxième stratégie à étudier la convergence des polygones que l'on présentera dans les sections qui suivent.

On fixe dans cette section une application $f: \mathbb{N} \rightarrow \mathbb{R}_{\geqslant 0}$ et une algèbre graduée $B=\bigoplus_{n \geqslant 0} B_{n}$ sur $k$, où chaque espace vectoriel $B_{n}$ est de rang fini et est muni d'une $\mathbb{R}$-filtration décroissante $\mathcal{F}^{(n)}$.

\subsubsection{Algèbre graduée quasi-filtrée}

DÉFInition 3.2.1. - On dit que l'algèbre graduée $B$ est $f$-quasi-filtrée s'il existe un entier $n_{0} \geqslant 0$ tel que, pour tout entier $r>0$, tout $\left(n_{i}\right)_{1 \leqslant i \leqslant r} \in \mathbb{Z}_{\geqslant n_{0}}^{r}$ et tout $\left(s_{i}\right)_{1 \leqslant i \leqslant r} \in$ $\mathbb{R}^{r}$, on ait

$$
\prod_{i=1}^{r} \mathcal{F}_{s_{i}}^{\left(n_{i}\right)} B_{n_{i}} \subset \mathcal{F}_{S}^{(N)} B_{N}, \quad \text { où } \quad N=\sum_{i=1}^{r} n_{i} \quad \text { et } \quad S=\sum_{i=1}^{r}\left(s_{i}-f\left(n_{i}\right)\right) .
$$

Une algèbre graduée 0 -quasi-filtrée avec $n_{0}=0$ s'appelle aussi une algèbre graduée filtrée, où le symbole 0 désigne la fonction constante qui prend valeur 0 .

L'algèbre graduée $B$ est $f$-quasi-filtrée si et seulement s'il existe un entier $n_{0} \geqslant 0$ tel que, pour tous les éléments homogènes $x_{1}, \ldots, x_{r}$ de degré $n_{1}, \ldots, n_{r}$ dans $\mathbb{N}_{\geqslant n_{0}}$ respectivement, en posant $x=x_{1} x_{2} \cdots x_{r}$ et $N=n_{1}+\cdots+n_{r}$, on ait

$$
\lambda_{\mathscr{F}^{(N)}}(x) \geqslant \sum_{i=1}^{r}\left(\lambda_{\mathscr{F}^{\left(n_{i}\right)}}\left(x_{i}\right)-f\left(n_{i}\right)\right) \text {. }
$$

Exemple 3.2.2. - On considère un exemple où l'algèbre $B$ est l'algèbre $k[X]$ des polynôme à une variable, munie de la graduation usuelle. Pour tout entier $n \geqslant 1$ soit $a_{n}=\lambda_{\mathcal{F}^{(n)}}\left(X^{n}\right)$. Alors cette algèbre graduée est $f$-quasi-filtrée si et seulement s'il existe un entier $n_{0} \geqslant 0$ tel que, pour toute suite finie $n_{1}, \ldots, n_{r}$ d'entiers $\geqslant n_{0}$, on ait

$$
a_{n_{1}+\cdots+n_{r}} \geqslant \sum_{i=1}^{r}\left(a_{n_{i}}-f\left(n_{i}\right)\right) .
$$

D'après le corollaire 1.3 .2 , la suite $\left(a_{n} / n\right)_{n \geqslant 1}$ converge pourvu que $a_{n}=O(n)(n \rightarrow$ $\infty)$ et que $\lim _{n \rightarrow+\infty} f(n) / n=0$. 
Remarque 3.2.3. - On suppose que l'algèbre graduée $B$ est $f$-quasi-filtrée. Alors pour toute fonction $g$ qui domine $f$, c'est-à-dire telle que $g(n) \geqslant f(n)$ quel que soit $n \in \mathbb{N}$, l'algèbre $B$ est $g$-quasi-filtrée. En outre, pour toute sous-algèbre $A$ engendrée par des éléments homogènes, $A$ est aussi $f$-quasi-filtrée.

On présente au-dessous un résultat de convergence. Bien que sa démonstration est élémentaire, elle contient déjà des idées importantes de la preuve de la convergence des polygones.

Proposition 3.2.4. - On suppose que

1) l'algèbre graduée $B$ est intègre et $f$-quasi-filtrée, et $B_{n} \neq 0$ pour $n$ assez grand,

2) $\lim _{n \rightarrow+\infty} f(n) / n=0$,

3) il existe $\alpha>0$ tel que $\lambda_{\max }\left(B_{n}, \mathcal{F}^{(n)}\right) \leqslant \alpha n$ pour tout entier $n \geqslant 1$.

Alors la suite $\left(\lambda_{\max }\left(B_{n}, \mathcal{F}^{(n)}\right) / n\right)_{n \geqslant 1}$ converge dans $\mathbb{R}$, où l'application $\lambda_{\max }$ est définie dans (3).

Démonstration. - Soit $n_{0} \geqslant 0$ comme dans la définition 3.2.1. On peut supposer $B_{n} \neq 0$ pour tout $n \geqslant n_{0}$. Pour tout entier $n \geqslant n_{0}$, soit $x_{n}$ un élément non-nul dans $B_{n}$ tel que $\lambda_{\mathscr{F}^{(n)}}\left(x_{n}\right)=\lambda_{\max }\left(B_{n}, \mathcal{F}^{(n)}\right)$. On suppose que $r \geqslant 1$ est un entier et que $n_{1}, \ldots, n_{r}$ sont des éléments dans $\mathbb{Z}_{\geqslant n_{0}}$. On note $N=n_{1}+\cdots+n_{r}$. L'algèbre $B$ étant intègre, le produit $x_{n_{1}} \cdots x_{n_{r}}$ est non-nul. Comme $B$ est $f$-quasi-filtrée, on a

$$
\begin{aligned}
\lambda_{\max }\left(B_{N}, \mathcal{F}^{(N)}\right) \geqslant & \lambda_{\mathcal{F}^{(N)}}\left(x_{n_{1}} \cdots x_{n_{r}}\right) \geqslant \sum_{i=1}^{r}\left(\lambda_{\mathcal{F}^{\left(n_{i}\right)}}\left(x_{n_{i}}\right)-f\left(n_{i}\right)\right) \\
& =\sum_{i=1}^{r}\left(\lambda_{\max }\left(B_{n_{i}}, \mathcal{F}^{\left(n_{i}\right)}\right)-f\left(n_{i}\right)\right) .
\end{aligned}
$$

Par conséquent, la suite $\left(\lambda_{\max }\left(B_{n}, \mathcal{F}^{(n)}\right)\right)_{n \geqslant 1}$ vérifie les conditions du corollaire 1.3.2. Donc elle converge dans $\mathbb{R}$.

Remarque 3.2.5. - On verra dans la remarque 3.4.4 que l'énoncé de la proposition 3.2.4 n'est pas nécessairement vrai si l'algèbre $B$ n'est pas intègre.

Proposition 3.2.6. - On suppose que

1) l'algèbre graduée $B$ est $f$-quasi-filtrée, $B_{n} \neq 0$ pour $n$ assez grand, et $B_{n} B_{m}=$ $B_{n+m}$ pour $n$ et $m$ assez grands,

2) $\lim _{n \rightarrow+\infty} f(n) / n=0$,

3) il existe $\alpha>0$ tel que $\lambda_{\min }\left(B_{n}, \mathcal{F}^{(n)}\right) \leqslant \alpha n$ pour tout entier $n \geqslant 1$.

Alors la suite $\left(\lambda_{\min }\left(B_{n}, \mathcal{F}^{(n)}\right) / n\right)_{n \geqslant 1}$ converge dans $\mathbb{R}$, où l'application $\lambda_{\min }$ est définie dans (3). 
Démonstration. - Soit $n_{0} \geqslant 0$ comme dans la définition 3.2.1. Quitte à agrandir $n_{0}$, on peut supposer que $B_{n} B_{m}=B_{n+m}$ dès que $n$ et $m$ sont tous supérieurs ou égaux à $n_{0}$. Soient $n_{1}, \ldots, n_{r}$ des entiers arbitraires dans $\mathbb{Z}_{\geqslant n_{0}}$ et $N=n_{1}+\cdots+n_{r}$. Soit $W=\left\{b_{1} \cdots b_{r} \mid \forall i, b_{i} \in B_{n_{i}}\right\}$. Comme $B_{N}=B_{n_{1}} \cdots B_{n_{r}}$, l'espace $B_{N}$ est engendré comme groupe additif par $W$. Si $a_{1}, \ldots, a_{m}$ sont des éléments dans $W$, on a $\lambda_{\mathscr{F}^{(N)}}\left(a_{1}+\cdots+a_{m}\right) \geqslant \min _{1 \leqslant i \leqslant m} \lambda_{\mathscr{F}^{(N)}}\left(a_{i}\right)$. On en déduit l'existence d'un élément $y \in W$ tel que $\lambda_{\mathscr{F}^{(N)}}(y)=\lambda_{\min }\left(B_{N}, \mathcal{F}^{(N)}\right)$. On suppose que $y=c_{1} \cdots c_{r}$ avec $c_{i} \in B_{n_{i}}$. Alors

$$
\begin{aligned}
\lambda_{\min }\left(B_{N}, \mathcal{F}^{(N)}\right) & =\lambda_{\mathcal{F}^{(N)}}(y) \geqslant \sum_{i=1}^{r}\left(\lambda_{\mathcal{F}^{\left(n_{i}\right)}}\left(c_{i}\right)-f\left(n_{i}\right)\right) \\
& \geqslant \sum_{i=1}^{r}\left(\lambda_{\min }\left(B_{n_{i}}, \mathcal{F}^{\left(n_{i}\right)}\right)-f\left(n_{i}\right)\right) .
\end{aligned}
$$

D'après le corollaire 1.3.2, la suite $\left(\lambda_{\min }\left(B_{n}, \mathcal{F}^{(n)}\right) / n\right)_{n \geqslant 1}$ converge dans $\mathbb{R}$.

REMARQUE 3.2.7. - L'assertion de la proposition 3.2.6 n'est pas nécessairement vraie si la condition " $B_{n+m}=B_{n} B_{m}$ pour $n, m$ assez grands" n'est pas satisfaite. On considère l'algèbre de polynômes $B=k[X, Y]$ qui est graduée de sorte que $X$ soit homogène de degré 1 et $Y$ soit homogène de degré 2 . Dans ce cas-là $B_{n}$ est engendré comme un espace vectoriel sur $k$ par $\mathbf{e}_{n}:=\left(X^{n}, X^{n-2} Y, \ldots, Y^{n / 2}\right)$ si $n$ est pair et par $\mathbf{e}_{n}:=\left(X^{n}, X^{n-2} Y, \ldots, X Y^{(n-1) / 2}\right)$ si $n$ est impaire. Pour tout entier $n \geqslant 1$, on munit l'espace $B_{n}$ d'une filtration $\mathcal{F}^{(n)}$ telle que

1) la base $\mathbf{e}_{n}$ soit compatible à $\mathscr{F}^{(n)}$

2) si $n$ est impair, pour tout $a \in \mathbf{e}_{n}, \lambda_{\mathscr{F}^{(n)}}(a)=0$;

3) si $n$ est pair, pour tout $a \in \mathbf{e}_{n}$ autre que $Y^{n / 2}, \lambda_{\mathscr{F}^{(n)}}(a)=0$, et $\lambda_{\mathscr{F}^{(n)}}\left(Y^{n / 2}\right)=-n$.

On vérifie que l'algèbre $B$ est filtrée. D'abord, pour tout élément $Q \in B_{n}, \lambda_{\mathcal{F}^{(n)}}(Q)=$ 0 si $Q$ est divisible par $X$ et $\lambda_{\mathcal{F}^{(n)}}(Q)=-n$ sinon. On suppose que $P \in B_{n}$ et $Q \in B_{m}$ sont deux éléments non-nuls. Si l'un des $P$ et $Q$ est divisible par $X$, alors il en est de même de $P Q$. Par conséquent, $\lambda_{\mathcal{F}^{(n+m)}}(P Q)=0 \geqslant \lambda_{\mathcal{G}^{(n)}}(P)+\lambda_{\mathcal{G}^{(n)}}(Q)$; sinon $\lambda_{\mathscr{G}^{(n+m)}}(P Q)=-(n+m)=\lambda_{\mathscr{G}^{(n)}}(P)+\lambda_{\mathscr{G}^{(m)}}(Q)$. Donc la relation $\lambda_{\mathscr{G}^{(n+m)}}(P Q) \geqslant$ $\lambda_{\mathcal{F}^{(n)}}(P)+\lambda_{\mathcal{F}^{(n)}}(Q)$ est toujours vérifiée. On en déduit que l'algèbre graduée $B$ est filtrée. Cependant, $\lambda_{\min }\left(B_{n}, \mathcal{F}^{(n)}\right)$ est égal à 0 si $n$ est impair et est égal à $-n$ si $n$ est pair. La suite $\left(\lambda_{\min }\left(B_{n}, \mathscr{F}^{(n)}\right) / n\right)_{n \geqslant 1}$ ne converge pas.

Bien que les hypothèses algébriques dans les propositions 3.2.4 et 3.2.6 sont légèrement différentes, les preuves utilisent une même technique - les suites presque sur-additives - discutée dans $\S 1.3$, qui jouera un rôle important dans l'étude de la convergence des polygones. 
3.2.2. Algèbre graduée pseudo-filtrée. - On présente une variante de la notion d'algèbre graduée quasi-filtrée - celle d'algèbre graduée pseudo-filtrée. Cette notion demande des conditions algébriques plus faibles. Ceci permet de simplifier dans certains cas la vérification des conditions.

DÉFInition 3.2.8. - On dit que l'algèbre graduée $B$ est $f$-pseudo-filtrée s'il existe un entier $n_{0} \geqslant 0$ tel que, pour tout couple d'entiers $(m, n) \in \mathbb{Z}_{\geqslant n_{0}}^{2}$, tout $(s, t) \in \mathbb{R}^{2}$, on ait $\left(\mathcal{F}_{s}^{(m)} B_{m}\right)\left(\mathcal{F}_{t}^{(n)} B_{n}\right) \subset \mathcal{F}_{s+t-f(m)-f(n)}^{(m+n)} B_{m+n}$.

Une algèbre graduée 0-pseudo-filtrée avec $n_{0}=0$ est exactement une algèbre graduée filtrée. En outre, l'algèbre graduée $B$ est pseudo-filtrée si et seulement s'il existe un entier $n_{0} \geqslant 0$ tel que, pour tous les éléments homogènes $x$ et $y$ de $B$ de degrés $m$ et $n$ dans $\mathbb{Z}_{\geqslant n_{0}}$ respectivement, on ait $\lambda_{\mathscr{F}^{(m+n)}}(x y) \geqslant \lambda_{\mathscr{F}^{(m)}}(x)+\lambda_{\mathscr{F}^{(n)}}(y)-$ $f(m)-f(n)$.

On présente dans le contexte des algèbres graduées pseudo-filtrées les résultats de convergence des suites $\left(\lambda_{\max }\left(B_{n}, \mathcal{F}^{(n)}\right) / n\right)_{n \geqslant 1}$ et $\left(\lambda_{\min }\left(B_{n}, \mathcal{F}^{(n)}\right) / n\right)$ en s'imposant d'une condition de croissante lente de $f$ qui est plus forte que l'annulation de la limite $\lim _{n \rightarrow+\infty} f(n) / n$. Au lieu d'utiliser le corollaire 1.3.2, on fait appel au corollaire 1.3.6. Les démonstrations sont très similiares à celles des propositions 3.2.4 et 3.2.6.

Proposition 3.2.9. - On suppose que

1) l'algèbre graduée $B$ est intègre et $f$-pseudo-filtrée, et $B_{n} \neq 0$ pour $n$ assez grand,

2) la fonction $f$ est croissante et $\sum_{\alpha \geqslant 0} f\left(2^{\alpha}\right) / 2^{\alpha}<+\infty$,

3) il existe $\alpha>0$ tel que $\lambda_{\max }\left(B_{n}, \mathcal{F}^{(n)}\right) \leqslant \alpha n$ pour tout entier $n \geqslant 1$.

Alors la suite $\left(\lambda_{\max }\left(B_{n}, \mathcal{F}^{(n)}\right) / n\right)_{n \geqslant 1}$ converge dans $\mathbb{R}$.

Proposition 3.2.10. - On suppose que

1) l'algèbre graduée $B$ est $f$-pseudo-filtrée, $B_{n} \neq 0$ pour $n$ assez grand, et $B_{n} B_{m}=$ $B_{n+m}$ pour $n$ et $m$ assez grands,

2) la fonction $f$ est croissante et $\sum_{\alpha \geqslant 0} f\left(2^{\alpha}\right) / 2^{\alpha}<+\infty$,

3) il existe $\alpha>0$ tel que $\lambda_{\min }\left(B_{n}, \mathcal{F}^{(n)}\right) \leqslant \alpha n$ pour tout entier $n \geqslant 1$.

Alors la suite $\left(\lambda_{\min }\left(B_{n}, \mathcal{F}^{(n)}\right) / n\right)_{n \geqslant 1}$ converge dans $\mathbb{R}$.

\subsection{Convergence des mesures : cas d'une algèbre de polynômes}

On démontrera dans cette section la convergence vague des mesures associées à une algèbre de polynômes usuellement graduée qui est quasi-filtrée. Comme expliqué au début du chapitre, l'idée est de passer par la presque sur-additivité de ces mesures. Cette presque sur-additivité sera établie par un argument combinatoire fin des points entiers dans des simplexes. 
3.3.1. Combinatoire des points entiers dans des simplexes. - Pour tout couple d'entiers $(n, d)$ tel que $n \geqslant 0$ et que $d \geqslant 1$, soit $\Omega_{n}^{(d)}$ le sous-ensemble de $\mathbb{N}^{d}$ formé des décompositions de $n$ en somme de $d$ entiers positifs ou nuls. On introduit la relation d'ordre lexicographique sur $\Omega_{n}^{(d)}:\left(a_{1}, \ldots, a_{d}\right) \geqslant\left(b_{1}, \ldots, b_{d}\right)$ si et seulement s'il existe $i \in\{1, \ldots, d\}$ tel que $a_{j}=b_{j}$ pour tout $j \in\{1, \ldots, i\}$ et que $a_{i+1}>b_{i+1}$ si $i<d$. L'ensemble $\Omega_{n}^{(d)}$ est totalement ordonné par cette relation d'ordre. D'autre part, pour tout $\mathbf{n}=\left(n_{i}\right)_{1 \leqslant i \leqslant r} \in \mathbb{N}^{r}$, on a une application de $\Omega_{n_{1}}^{(d)} \times \cdots \times \Omega_{n_{r}}^{(d)}$ vers $\Omega_{n_{1}+\cdots+n_{r}}^{(d)}$, qui envoie $\left(\alpha_{i}\right)_{1 \leqslant i \leqslant r}$ vers $\alpha_{1}+\cdots+\alpha_{r}$ (l'addition étant celle de $\left.\mathbb{Z}^{d}\right)$. Cette application n'est pas injective en général mais est toujours surjective. En outre, si $\left(\alpha_{i}\right)_{1 \leqslant i \leqslant r}$ et $\left(\beta_{i}\right)_{1 \leqslant i \leqslant r}$ sont deux éléments de $\Omega_{n_{1}}^{(d)} \times \cdots \Omega_{n_{r}}^{(d)}$ tels que $\alpha_{i} \geqslant \beta_{i}$ pour tout $i$, alors $\alpha_{1}+\cdots+\alpha_{r} \geqslant \beta_{1}+\cdots+\beta_{r}$.

Pour tout $n \in \mathbb{N}$, on désigne par $\Gamma_{n}^{(d)}$ le sous-ensemble de $\mathbb{N}^{d-1}$ formé des éléments $\left(a_{i}\right)_{1 \leqslant i \leqslant d-1}$ tels que $0 \leqslant a_{1}+\cdots+a_{d-1} \leqslant n$. On a une bijection naturelle $p_{n}^{(d)}$ : $\Omega_{n}^{(d)} \rightarrow \Gamma_{n}^{(d)}$ définie par la projection sur les $d-1$ premiers facteurs. Son inverse est l'application qui envoie $\left(a_{i}\right)_{1 \leqslant i \leqslant d-1}$ en $\left(a_{1}, \ldots, a_{d-1}, n-a_{1}-\cdots-a_{d-1}\right)$. Pour tout $\mathbf{n}=\left(n_{i}\right)_{1 \leqslant i \leqslant r} \in \mathbb{N}^{r}$, le diagramme suivant est commutatif :

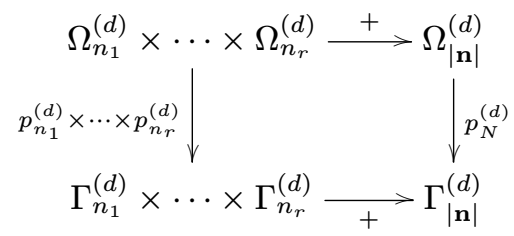

où $|\mathbf{n}|=n_{1}+\cdots+n_{r}$ et où les applications "+" sont définies par l'addition dans les monoïdes $\mathbb{N}^{d}$ et $\mathbb{N}^{d-1}$, respectivement.

ThÉorème 3.3.1. - Soient $r \geqslant 2$ et $d \geqslant 1$ deux entiers. Pour tout $\mathbf{n}=\left(n_{i}\right)_{1 \leqslant i \leqslant r} \in$ $\mathbb{N}^{r}$, il existe une mesure de probabilité $\rho_{\mathbf{n}}$ sur $\Omega_{n_{1}}^{(d)} \times \cdots \times \Omega_{n_{r}}^{(d)}$ telle que l'image directe de $\rho_{\mathbf{n}}$ par chacune des $r$ projections sur $\Omega_{n_{1}}^{(d)}, \ldots, \Omega_{n_{r}}^{(d)}$ soit une mesure équiprobable, ainsi que son image directe par l'application "+" à valeurs dans $\Omega_{|\mathbf{n}|}^{(d)}$.

Démonstration. - Le théorème est trivial lorsque $d=1$ car alors, pour tout $k \in \mathbb{N}$, $\Omega_{k}^{(d)}$ est le singleton $\{k\}$. Dans la suite de la démonstration, on suppose $d \geqslant 2$. D'après (52), il suffit de construire une mesure de probabilité $\rho_{\mathbf{n}}$ sur $\Gamma_{n_{1}}^{(d)} \times \cdots \times \Gamma_{n_{r}}^{(d)}$ telle que l'image directe de $\rho_{\mathbf{n}}$ par chacune des $r$ projections sur $\Gamma_{n_{1}}^{(d)}, \ldots, \Gamma_{n_{r}}^{d}$ soit une mesure équiprobable, ainsi que son image directe par l'application "+" à valeurs dans $\Gamma_{|\mathbf{n}|}^{(d)}$.

Pour tout $\alpha=\left(a_{i}\right)_{1 \leqslant i \leqslant d-1} \in \mathbb{N}^{d-1}$, on définit $|\alpha|=a_{1}+\cdots+a_{d-1}$. L'ensemble $\Gamma_{n}^{(d)}$ s'écrit sous la forme $\Gamma_{n}^{(d)}=\left\{\alpha \in \mathbb{N}^{d-1}|| \alpha \mid \leqslant n\right\}$. Si $\alpha=\left(a_{i}\right)_{1 \leqslant i \leqslant d-1}$ est un élément de $\mathbb{N}^{d-1}$, on note $\alpha !=a_{1} ! \times \cdots \times a_{d-1} !$.

On considère l'anneau des séries formelles de $r d$ variables $R=\mathbb{Z} \llbracket \mathbf{t}, \mathbf{X} \rrbracket$, où $\mathbf{t}=$ $\left(t_{1}, \ldots, t_{r}\right), \mathbf{X}=\left(X_{i, j}\right)_{\substack{1 \leqslant i \leqslant r \\ 1 \leqslant j \leqslant d-1}}$. Si $\alpha=\left(a_{1}, \ldots, a_{d-1}\right) \in \mathbb{N}^{d-1}$ et si $i \in\{1, \ldots, r\}$, on désigne par $X_{i}^{\alpha}$ le produit $X_{i, 1}^{a_{1}} \times \cdots \times X_{i, d-1}^{a_{d-1}}$. Si $\mathbf{n}=\left(n_{1}, \ldots, n_{r}\right)$ est un élément 
de $\mathbb{N}^{r}$, on désigne par $\mathbf{t}^{\mathbf{n}}$ le produit $t_{1}^{n_{1}} \times \cdots \times t_{r}^{n_{r}}$. Soit $H(\mathbf{t}, \mathbf{X})$ la série formelle à coefficients entiers positifs

$$
\sum_{\mathbf{n}=\left(n_{i}\right) \in \mathbb{N}^{r}} \mathbf{t}^{\mathbf{n}} \sum_{\substack{\left(\alpha_{i}\right) \in\left(\mathbb{N}^{d-1}\right)^{r} \\\left|\alpha_{i}\right| \leqslant n_{i}}} \frac{\left(\alpha_{1}+\cdots+\alpha_{r}\right) !}{\alpha_{1} ! \cdots \alpha_{r} !} \frac{\left(n_{1}+\cdots+n_{r}-\left|\alpha_{1}+\cdots+\alpha_{r}\right|\right) !}{\left(n_{1}-\left|\alpha_{1}\right|\right) ! \cdots\left(n_{r}-\left|\alpha_{r}\right|\right) !} \prod_{j=1}^{r} X_{j}^{\alpha_{j}} .
$$

En faisant les changements d'indexation $m_{i}=n_{i}-\left|\alpha_{i}\right|$ et en permutant les sommations, puis en posant $\left(\beta_{1}, \ldots, \beta_{d-1}\right)=\alpha_{1}+\cdots+\alpha_{r}$ et $m=m_{1}+\cdots+m_{r}$, on obtient l'égalité suivante dans $\mathbb{Z} \llbracket \mathbf{t}, \mathbf{X} \rrbracket$ :

$$
\begin{aligned}
H(\mathbf{t}, \mathbf{X}) & =\sum_{\left(\alpha_{i}\right) \in\left(\mathbb{N}^{d-1}\right)^{r}} \frac{\left(\alpha_{1}+\cdots+\alpha_{r}\right) !}{\alpha_{1} ! \cdots \alpha_{r} !} \prod_{j=1}^{r} t_{j}^{\left|\alpha_{j}\right|} X_{j}^{\alpha_{j}} \sum_{\mathbf{m}=\left(m_{i}\right) \in \mathbb{N}^{r}} \frac{\left(m_{1}+\cdots+m_{r}\right) !}{m_{1} ! \cdots m_{r} !} \mathbf{t}^{\mathbf{m}} \\
& =\sum_{\left(\beta_{i}\right) \in \mathbb{N}^{d-1}} \prod_{i=1}^{d-1}\left(t_{1} X_{1, i}+\cdots+t_{r} X_{r, i}\right)^{\beta_{i}} \sum_{m \in \mathbb{N}}\left(t_{1}+\cdots+t_{r}\right)^{m} \\
& =\left(1-\left(t_{1}+\cdots+t_{r}\right)\right)^{-1} \prod_{i=1}^{d-1}\left(1-\left(t_{1} X_{1, i}+\cdots+t_{r} X_{r, i}\right)\right)^{-1} .
\end{aligned}
$$

Ce calcul montre aussi (cf. [31] II §2.4) que le domaine de convergence absolue de Reinhardt de $H(\mathbf{t}, \mathbf{X})$ dans $\mathbb{C}^{r d}$ est défini par les conditions

$$
\sum_{j=1}^{r}\left|t_{j}\right|<1 \quad \text { et } \quad \sum_{j=1}^{r}\left|t_{j}\right|\left|X_{j, i}\right|<1 .
$$

Cette observation nous autorise à substituer le vecteur $\mathbb{1}=(\underbrace{1, \ldots, 1}_{d-1 \text { copies }})$ à certaines des variables $X_{i}$ sans avoir à examiner de questions de convergence (qui sont en fait anodines).

On effectue le changement de variables $m_{i}=n_{i}-\left|\alpha_{i}\right|$ pour $2 \leqslant i \leqslant r$, et on obtient

$$
\begin{aligned}
&\left.H(\mathbf{t}, \mathbf{X})\right|_{X_{2}=\cdots=X_{r}=\mathbb{1}} \sum_{n_{1} \geqslant 0} t_{1}^{n_{1}} \sum_{\left|\alpha_{1}\right| \leqslant n_{1}} X_{1}^{\alpha_{1}} \sum_{\begin{array}{c}
\left(\alpha_{i}\right)_{i=2}^{r} \in\left(\mathbb{N}^{d-1}\right)^{r-1} \\
\left(m_{i}\right)_{i=2}^{r} \in \mathbb{N}^{r-1}
\end{array}} \frac{\left(\alpha_{1}+\cdots+\alpha_{r}\right) !}{\alpha_{1} ! \cdots \alpha_{r} !} \\
& \frac{\left(n_{1}+m_{2}+\cdots+m_{r}-\left|\alpha_{1}\right|\right) !}{\left(n_{1}-\left|\alpha_{1}\right|\right) ! m_{2} ! \cdots m_{r} !} \prod_{j=2}^{r} t_{j}^{m_{j}+\left|\alpha_{j}\right|} \\
&= \sum_{n_{1} \geqslant 0} t_{1}^{n_{1}} \sum_{\left|\alpha_{1}\right| \leqslant n_{1}} X_{1}^{\alpha_{1}} \sum_{\left(\alpha_{i}\right)_{i=2}^{r} \in\left(\mathbb{N}^{d-1}\right)^{r-1}} \frac{\left(\alpha_{1}+\cdots+\alpha_{r}\right) !}{\alpha_{1} ! \cdots \alpha_{r} !} \prod_{j=2}^{r} t_{j}^{\left|\alpha_{j}\right|} \\
& \sum_{\left(m_{i}\right)_{i=2}^{r} \in \mathbb{N}^{r-1}} \frac{\left(n_{1}+m_{2}+\cdots+m_{r}-\left|\alpha_{1}\right|\right) !}{\left(n_{1}-\left|\alpha_{1}\right|\right) ! m_{2} ! \cdots m_{r} !} \prod_{j=2}^{r} t_{j}^{m_{j}} .
\end{aligned}
$$


Pour tout $a \in \mathbb{N}$, l'égalité suivante est vérifiée dans $\mathbb{Z} \llbracket t \rrbracket$

$$
\sum_{b \geqslant 0} \frac{(a+b) !}{a ! b !} t^{b}=(1-t)^{-a-1} .
$$

On en déduit donc

$$
\begin{aligned}
\sum_{\left(\alpha_{i}\right)_{i=2}^{r} \in\left(\mathbb{N}^{d-1}\right)^{r-1}} & \frac{\left(\alpha_{1}+\cdots+\alpha_{r}\right) !}{\alpha_{1} ! \cdots \alpha_{r} !} \prod_{j=2}^{r} t_{j}^{\left|\alpha_{j}\right|} \\
= & \sum_{\left(\alpha_{i}\right)_{i=2}^{r} \in\left(\mathbb{N}^{d-1}\right)^{r-1}} \frac{\left(\alpha_{1}+\cdots+\alpha_{r}\right) !}{\alpha_{1} !\left(\alpha_{2}+\cdots+\alpha_{r} !\right)} \frac{\left(\alpha_{2}+\cdots+\alpha_{r}\right) !}{\alpha_{2} ! \cdots \alpha_{r} !} \prod_{j=2}^{r} t_{j}^{\left|\alpha_{j}\right|} \\
= & \sum_{\alpha \in \mathbb{N}^{d-1}} \frac{\left(\alpha_{1}+\alpha\right) !}{\alpha_{1} ! \alpha !} \sum_{\substack{\left(\alpha_{i}\right)_{i=2}^{r} \in\left(\mathbb{N}^{d-1}\right)^{r-1} \\
\alpha_{2}+\cdots+\alpha_{r}=\alpha}} \frac{\left(\alpha_{2}+\cdots+\alpha_{r}\right) !}{\alpha_{1} ! \cdots \alpha_{r} !} \prod_{j=2}^{r} t_{j}^{\left|\alpha_{j}\right|} \\
= & \sum_{\alpha \in \mathbb{N}^{d-1}} \frac{\left(\alpha_{1}+\alpha\right) !}{\alpha_{1} ! \alpha !}\left(t_{2}+\cdots+t_{r}\right)^{|\alpha|}=\left(1-\left(t_{2}+\cdots+t_{r}\right)\right)^{-\left|\alpha_{1}\right|-d+1},
\end{aligned}
$$

et

$$
\begin{aligned}
\sum_{\left(m_{i}\right)_{i=2}^{r} \in \mathbb{N}^{r-1}} & \frac{\left(n_{1}+m_{2}+\cdots+m_{r}-\left|\alpha_{1}\right|\right) !}{\left(n_{1}-\left|\alpha_{1}\right|\right) ! m_{2} ! \cdots m_{r} !} \prod_{j=2}^{r} t_{j}^{m_{j}} \\
& =\sum_{\left(m_{i}\right)_{i=2}^{r} \in \mathbb{N}^{r-1}} \frac{\left(n_{1}+m_{2}+\cdots+m_{r}-\left|\alpha_{1}\right|\right) !}{\left(n_{1}-\left|\alpha_{1}\right|\right) !\left(m_{2}+\cdots+m_{r}\right) !} \frac{\left(m_{2}+\cdots+m_{r}\right) !}{m_{2} ! \cdots m_{r} !} \prod_{j=2}^{r} t_{j}^{m_{j}} \\
& =\sum_{M \geqslant 0} \frac{\left(n_{1}-\left|\alpha_{1}\right|+M\right) !}{\left(n_{1}-\left|\alpha_{1}\right|\right) ! M !} \sum_{\left(m_{i}\right)_{i=2}^{r} \in \mathbb{N}^{r-1}} \frac{\left(m_{2}+\cdots+m_{r}\right) !}{m_{2} ! \cdots m_{r} !} \prod_{j=2}^{r} t_{j}^{m_{j}} \\
& =\sum_{M \geqslant 0} \frac{\left(n_{1}-\left|\alpha_{1}\right|+M\right) !}{\left(n_{1}-\left|\alpha_{1}\right|\right) ! M !}\left(t_{2}+\cdots+t_{r}\right)^{M}=\left(1-\left(t_{2}+\cdots+t_{r}\right)\right)^{-n_{1}+\left|\alpha_{1}\right|-1} .
\end{aligned}
$$

Par conséquent,

$$
\begin{aligned}
\left.H(\mathbf{t}, \mathbf{X})\right|_{X_{2}=\cdots=X_{r}=\mathbb{1}} & =\sum_{n_{1} \geqslant 0} t_{1}^{n_{1}}\left(1-\left(t_{2}+\cdots+t_{r}\right)\right)^{-n_{1}-d} \sum_{\left|\alpha_{1}\right| \leqslant n_{1}} X_{1}^{\alpha_{1}} \\
& =\sum_{\mathbf{n}=\left(n_{i}\right) \in \mathbb{N}^{r}} \mathbf{t}^{\mathbf{n}} \frac{\left(n_{1}+\cdots+n_{r}+d-1\right) !}{\left(n_{1}+d-1\right) ! n_{2} ! \cdots n_{r} !} \sum_{\left|\alpha_{1}\right| \leqslant n_{1}} X_{1}^{\alpha_{1}} .
\end{aligned}
$$

De même, pour tout $j \in\{1, \ldots, r\}$, on a

$$
\begin{aligned}
\left.H(\mathbf{t}, \mathbf{X})\right|_{X_{1}=\cdots=X_{j-1}=X_{j+1}=\cdots=X_{r}=\mathbb{1}} & \\
& =\sum_{\mathbf{n}=\left(n_{i}\right) \in \mathbb{N}^{r}} \mathbf{t}^{\mathbf{n}} \frac{\left(n_{1}+\cdots+n_{r}+d-1\right) !}{n_{1} ! \cdots n_{j-1} !\left(n_{j}+d-1\right) ! n_{j+1} ! \cdots n_{r} !} \sum_{\left|\alpha_{j}\right| \leqslant n_{j}} X_{j}^{\alpha_{j}} .
\end{aligned}
$$


D'autre part,

$$
\begin{aligned}
\left.H(\mathbf{t}, \mathbf{X})\right|_{X_{1}=\cdots=X_{r}=Y}= & \sum_{\mathbf{n}=\left(n_{i}\right) \in \mathbb{N}^{r}} \mathbf{t}^{\mathbf{n}} \sum_{\substack{\left(\alpha_{i}\right) \in\left(\mathbb{N}^{d-1}\right)^{r} \\
\left|\alpha_{i}\right| \leqslant n_{i}}} Y^{\alpha_{1}+\cdots+\alpha_{r}} \\
& \frac{\left(\alpha_{1}+\cdots+\alpha_{r}\right) !}{\alpha_{1} ! \cdots \alpha_{r} !} \frac{\left(n_{1}+\cdots+n_{r}-\left|\alpha_{1}+\cdots+\alpha_{r}\right|\right) !}{\left(n_{1}-\left|\alpha_{1}\right|\right) ! \cdots\left(n_{r}-\left|\alpha_{r}\right|\right) !} .
\end{aligned}
$$

En faisant les changements d'indexation $m_{i}=n_{i}-\left|\alpha_{i}\right|$ pour tout $1 \leqslant i \leqslant r$, on obtient

$$
\begin{aligned}
& \left.H(\mathbf{t}, \mathbf{X})\right|_{X_{1}=\cdots=X_{r}=Y} \\
= & \sum_{\left(\alpha_{i}\right) \in\left(\mathbb{N}^{d-1}\right)^{r}} \frac{\left(\alpha_{1}+\cdots+\alpha_{r}\right) !}{\alpha_{1} ! \cdots \alpha_{r} !} \prod_{j=1}^{r} t_{j}^{\left|\alpha_{j}\right|} Y^{\alpha_{1}+\cdots+\alpha_{r}} \sum_{\mathbf{m}=\left(m_{i}\right) \in \mathbb{N}^{r}} \frac{\left(m_{1}+\cdots+m_{r}\right) !}{m_{1} ! \cdots m_{r} !} \mathbf{t}^{\mathbf{m}} \\
= & \sum_{N \geqslant 0}\left(t_{1}+\cdots+t_{r}\right)^{N} \sum_{\gamma \in \mathbb{N}^{d-1}} Y^{\gamma}\left(t_{1}+\cdots+t_{r}\right)^{|\gamma|}=\sum_{M \geqslant 0}\left(t_{1}+\cdots+t_{r}\right)^{M} \sum_{|\gamma| \leqslant M} Y^{\gamma},
\end{aligned}
$$

où on a utilisé le changement d'indexation $M=N+|\gamma|$ dans la dernière égalité. Par conséquent,

$$
\left.H(\mathbf{t}, \mathbf{X})\right|_{X_{1}=\cdots=X_{r}=Y}=\sum_{\mathbf{n}=\left(n_{i}\right) \in \mathbb{N}^{r}} \frac{\left(n_{1}+\cdots+n_{r}\right) !}{n_{1} ! \cdots n_{r} !} t_{1}^{n_{1}} \cdots t_{r}^{n_{r}} \sum_{|\gamma| \leqslant n_{1}+\cdots+n_{r}} Y^{\gamma}
$$

Enfin,

$$
\begin{aligned}
H(\mathbf{t},(\mathbb{1}, \cdots \mathbb{1})) & =\left(1-\left(t_{1}+\cdots+t_{r}\right)\right)^{-d} \\
& =\sum_{N \geqslant 0} \frac{(N+d-1) !}{N !(d-1) !} \sum_{\substack{\mathbf{n}=\left(n_{i}\right) \in \mathbb{N}^{r} \\
n_{1}+\cdots+n_{r}=N}} \frac{N !}{n_{1} ! \cdots n_{r} !} \mathbf{t}^{\mathbf{n}} \\
& =\sum_{\mathbf{n}=\left(n_{i}\right) \in \mathbb{N}^{r}} \frac{\left(n_{1}+\cdots+n_{r}+d-1\right) !}{n_{1} ! \cdots n_{r} !(d-1) !} \mathbf{t}^{\mathbf{n}} .
\end{aligned}
$$

Pour tout $\mathbf{n}=\left(n_{i}\right) \in \mathbb{N}^{r}$, posons

$$
\begin{aligned}
\rho_{\mathbf{n}}= & \frac{(d-1) ! n_{1} ! \cdots n_{r} !}{\left(n_{1}+\cdots+n_{r}+d-1\right) !} \sum_{\substack{\left(\alpha_{i}\right) \in\left(\mathbb{N}^{d-1}\right)^{r} \\
\left|\alpha_{i}\right| \leqslant n_{i}}} \\
& \left(\frac{\left(\alpha_{1}+\cdots+\alpha_{r}\right) !}{\alpha_{1} ! \cdots \alpha_{r} !} \frac{\left(n_{1}+\cdots+n_{r}-\left|\alpha_{1}+\cdots+\alpha_{r}\right|\right) !}{\left(n_{1}-\left|\alpha_{1}\right|\right) ! \cdots\left(n_{r}-\left|\alpha_{r}\right|\right) !}\right) \delta_{\left(\alpha_{1}, \ldots, \alpha_{r}\right)} .
\end{aligned}
$$

La définition de $H(\mathbf{t}, \mathbf{X})$ et les égalités (54), (55) et (56) montrent que $\rho_{\mathbf{n}}$ vérifie les conditions requises.

REMARque 3.3.2. - Le théorème 3.3.1 équivaut à résoudre un système d'équations linéaires homogènes, où le nombre des indéterminés est en général beaucoup plus grand que celui des équations. La difficulté provient du besoin d'une solution positive et non-triviale. Un cas très particulier où $d=2 \mathrm{du}$ théorème montre que, pour tous les entiers $m, n \geqslant 1$, dans un réseau rectangulaire de taille $m \times n$, on peut toujours 
placer des entiers strictement positifs tels que, les sommes des entiers dans chaque ligne, dans chaque colonne et dans chaque diagonale principale sont respectivement égales.

3.3.2. Convergence vague des mesures. - Dans ce sous-paragraphe, on utilise le théorème combinatoire établi au-dessus pour démontrer la convergence vague des mesures. On fixe une algèbre de polynômes $B=k\left[X_{1}, \ldots, X_{d}\right](d \geqslant 1)$ qui est usuellement graduée, c'est-à-dire que $B_{n}$ est l'espaces des polynômes homogènes de degré $n$ en $X_{1}, \ldots, X_{d}$. Si $\alpha=\left(\alpha_{1}, \ldots, \alpha_{d}\right)$ est un élément dans $\mathbb{N}^{d}$, on utilise l'expression $X^{\alpha}$ pour désigner le monôme $X_{1}^{\alpha_{1}} \cdots X_{d}^{\alpha_{d}}$. Pour tout entier $n \geqslant 1$, on munit l'espace $B_{n}$ d'une $\mathbb{R}$-filtration $\mathcal{F}^{(n)}$. Enfin, soit $f: \mathbb{N} \rightarrow \mathbb{R}_{\geqslant 0}$ une application. On suppose que l'algèbre graduée $B$ est $f$-quasi-filtrée. Soit $n_{0}$ comme dans la définition 3.2.1. On suppose en outre $n_{0}>0$.

Soit $\varphi_{n}: \Omega_{n}^{(d)} \rightarrow B_{n}$ l'application qui envoie $\alpha=\left(\alpha_{1}, \ldots, \alpha_{d}\right)$ en $X^{\alpha}$, où $\Omega_{n}^{(d)}$ est l'ensemble des décompositions de $n$ défini dans le sous-paragraphe précédent. L'image de $\Omega_{n}^{(d)}$ par $\varphi_{n}$ est une base de $B_{n}$. D'après la proposition 1.2.4), il existe, pour tout $n \in \mathbb{N}$, une base $\mathbf{u}^{(n)}=\left(u_{\alpha}\right)_{\alpha \in \Omega_{n}^{(d)}}$ de $B_{n}$ telle que,

$$
\forall \alpha \in \Omega_{n}^{(d)}, \quad u_{\alpha} \in X^{\alpha}+\sum_{\beta<\alpha} k X^{\beta},
$$

et qui est compatible à la filtration $\mathcal{F}^{(n)}$. Si $\mathbf{n}=\left(n_{i}\right)_{1 \leqslant i \leqslant r} \in \mathbb{N}^{r}$ et si $N=n_{1}+\cdots+n_{r}$, pour tout $\gamma \in \Omega_{N}^{(d)}$, soit $u_{\gamma}^{(\mathbf{n})}$ un élément dans

$$
\left\{\prod_{i=1}^{r} u_{\alpha_{i}} \mid \alpha_{i} \in \Omega_{n_{i}}^{(d)}, \sum_{i=1}^{r} \alpha_{i}=\gamma\right\} .
$$

tel que

$$
\lambda_{\mathscr{F}^{(N)}}\left(u_{\gamma}^{(\mathbf{n})}\right)=\max _{\substack{\alpha_{i} \in \Omega_{n_{i}}^{(d)} \\ \alpha_{1}+\cdots+\alpha_{r}=\gamma}} \lambda_{\mathcal{F}^{(N)}}\left(u_{\alpha_{1}} \cdots u_{\alpha_{r}}\right) .
$$

De (57), on déduit

$$
u_{\gamma}^{(\mathbf{n})} \in X^{\gamma}+\sum_{\delta<\gamma} k X^{\delta} .
$$

Donc $\mathbf{u}^{(\mathbf{n})}:=\left(u_{\gamma}^{(\mathbf{n})}\right)_{\gamma \in \Omega_{N}^{(d)}}$ est une base de $B_{N}$.

Proposition 3.3.3. - Soient c un nombre réel positif et $g: \mathbb{R} \rightarrow \mathbb{R}$ une fonction croissante concave et c-lipschitzienne. Pour tout entier $n \geqslant 0$, soit

$$
I_{n}=\int_{\mathbb{R}} g \mathrm{~d}\left(T_{\frac{1}{n}} \nu_{\mathcal{F}^{(n)}}\right),
$$

où $\nu_{\mathcal{F}^{(n)}}$ est la mesure associée à la filtration $\mathcal{F}^{(n)}$ et $T_{\frac{1}{n}}$ est l'opération de dilatation définie dans \$1.2.4. Alors, pour tout entier $r \geqslant 2$ et tout $\mathbf{n}=\left(n_{i}\right) \in \mathbb{Z}_{\geqslant n_{0}}^{r}$, si on note 
$N=n_{1}+\cdots+n_{r}$, alors on a l'inégalité

$$
N I_{N} \geqslant \sum_{i=1}^{r}\left(n_{i} I_{n_{i}}-c f\left(n_{i}\right)\right)
$$

Démonstration. - Pour tout entier $n \geqslant 0$, on désigne par $\xi_{n}$ la mesure équiprobable sur $\Omega_{n}^{(d)}$, par $\rho_{\mathbf{n}}$ une mesure sur $\Omega_{n_{1}}^{(d)} \times \cdots \times \Omega_{n_{r}}^{(d)}$ satisfaisant aux conditions du théorème 3.3.1, et par $\mathbf{u}^{(\mathbf{n})}$ la base de $B_{N}$ construite comme ci-dessus. En utilisant les notations introduites dans $§ 1.2 .2$, on a l'inégalité suivante :

$$
\begin{aligned}
I_{N} & \geqslant \int_{\mathbb{R}} g \mathrm{~d}\left(T_{\frac{1}{N}} \nu_{\mathcal{F}^{(N)}}, \mathbf{u}^{(\mathbf{n})}\right)=\int_{\Omega_{N}^{(d)}} g\left(\frac{1}{N} \lambda_{\mathcal{F}^{(N)}}\left(u_{\gamma}^{(\mathbf{n})}\right)\right) \mathrm{d} \xi_{N}(\gamma) \\
& =\int_{\Omega_{n_{1}}^{(d)} \times \cdots \times \Omega_{n_{r}}^{(d)}} g\left(\frac{1}{N} \lambda_{\mathcal{F}^{(N)}}\left(u_{\alpha_{1}+\cdots+\alpha_{r}}^{(\mathbf{n})}\right)\right) \mathrm{d} \rho_{\mathbf{n}}\left(\alpha_{1}, \ldots, \alpha_{r}\right),
\end{aligned}
$$

où la dernière égalité est parce que l'image directe de $\rho_{\mathbf{n}}$ par l'addition est équiprobable. Comme $g$ est une fonction croissante, d'après la définition de $u_{\gamma}^{(\mathbf{n})}$, on a

$$
I_{N} \geqslant \int_{\Omega_{n_{1}}^{(d)} \times \cdots \times \Omega_{n_{r}}^{(d)}} g\left(\frac{1}{N} \lambda_{\mathcal{F}^{(N)}}\left(u_{\alpha_{1}}^{\left(n_{1}\right)} \cdots u_{\alpha_{r}}^{\left(n_{r}\right)}\right)\right) \mathrm{d} \rho_{\mathbf{n}}\left(\alpha_{1}, \ldots, \alpha_{n}\right) .
$$

Comme l'algèbre $B$ est graduée $f$-quasi-filtrée et comme $g$ est croissante,

$$
I_{N} \geqslant \int_{\Omega_{n_{1}}^{(d)} \times \cdots \times \Omega_{n_{r}}^{(d)}} g\left(\frac{1}{N} \sum_{i=1}^{r}\left(\lambda_{\mathcal{F}^{\left(n_{i}\right)}}\left(u_{\alpha_{i}}^{\left(n_{i}\right)}\right)-f\left(n_{i}\right)\right)\right) \mathrm{d} \rho_{\mathbf{n}}\left(\alpha_{1}, \ldots, \alpha_{r}\right) .
$$

Car la fonction $g$ est $c$-lipschitzienne,

$$
I_{N} \geqslant \int_{\Omega_{n_{1}}^{(d)} \times \cdots \times \Omega_{n_{r}}^{(d)}}\left[g\left(\frac{1}{N} \sum_{i=1}^{r} \lambda_{\mathscr{F}^{\left(n_{i}\right)}}\left(u_{\alpha_{i}}^{\left(n_{i}\right)}\right)\right)-\frac{c}{N} \sum_{i=1}^{r} f\left(n_{i}\right)\right] \mathrm{d} \rho_{\mathbf{n}}\left(\alpha_{1}, \ldots, \alpha_{r}\right) .
$$

Ensuite, la concavité de $g$ implique que

$$
I_{N} \geqslant \int_{\Omega_{n_{1}}^{(d)} \times \cdots \times \Omega_{n_{r}}^{(d)}}\left[\sum_{i=1}^{r} \frac{n_{i}}{N} g\left(\frac{\lambda_{\mathcal{F}^{\left(n_{i}\right)}}\left(u_{\alpha_{i}}^{\left(n_{i}\right)}\right)}{n_{i}}\right)\right] \mathrm{d} \rho_{\mathbf{n}}\left(\alpha_{1}, \ldots, \alpha_{r}\right)-\frac{c}{N} \sum_{i=1}^{r} f\left(n_{i}\right)
$$

Enfin, comme les images directes de $\rho_{\mathbf{n}}$ par les $r$ projections sont des mesures équiprobables, on obtient

$$
I_{N} \geqslant \sum_{i=1}^{r} \frac{n_{i}}{N} I_{n_{i}}-\frac{c}{N} \sum_{i=1}^{r} f\left(n_{i}\right) .
$$

Corollaire 3.3.4. - Avec les notations de la proposition 3.3.3, si la suite $\left(I_{n}\right)_{n \geqslant 0}$ est bornée supérieurement et si $\lim _{n \rightarrow+\infty} f(n) / n=0$, alors la suite $\left(I_{n}\right)_{n \geqslant 0}$ admet une limite dans $\mathbb{R}$ lorsque $n \rightarrow+\infty$.

Démonstration. - D'après la proposition 3.3.3, la suite $\left(n I_{n}\right)_{n \geqslant 1}$ vérifie les conditions du corollaire 1.3.2. On en déduit donc la convergence de la suite $\left(I_{n}\right)_{n \geqslant 0}$. 
REMARqUE 3.3.5. - La suite $\left(I_{n}\right)_{n \geqslant 1}$ est bornée supérieurement notamment lorsque la fonction $g$ est bornée supérieurement, ou lorsque $\lambda_{\max }\left(B_{n}, \mathcal{F}^{(n)}\right)=O(n)(n \rightarrow$ $+\infty)$.

On a établi dans le corollaire précédent la convergence des intégrales pour une famille particulière de fonctions définies sur $\mathbb{R}$. En fait, l'espace linéaire sur $\mathbb{R}$ engendré par cette famille de fonctions contient un sous-espace qui est dense dans $C_{c}(\mathbb{R})$ l'espace des fonctions continues à support compact. Cet argument permet de conclure la convergence vague des mesures.

Proposition 3.3.6. - Si $\lim _{n \rightarrow+\infty} f(n) / n=0$, alors la suite de mesures $\left(T_{\frac{1}{n}} \nu_{\mathcal{F}^{(n)}}\right)_{\geqslant 1}$ converge vaguement vers une mesure borélienne finie.

Démonstration. - Pour simplifier les notations, on note $\nu_{n}=T_{\frac{1}{n}} \nu_{\mathcal{F}^{(n)}}$. Soit $G$ l'ensemble des fonctions boréliennes $g$ sur $\mathbb{R}$ telle que, pour tout $n \in \mathbb{N}, g$ soit intégrable par rapport à $\nu_{n}$ et telle que la suite d'intégrales $\left(\int_{\mathbb{R}} g \mathrm{~d} \nu_{n}\right)_{n \geqslant 1}$ converge dans $\mathbb{R}$. Le corollaire 3.3.4 (voir aussi la remarque 3.3.5) montre que $G$ contient toutes les fonctions croissantes, concaves, lipschitziennes et bornées supérieurement. L'ensemble $G$ est un espace vectoriel sur $\mathbb{R}$. On suppose que $f$ est une fonction dans $C_{0}^{\infty}(\mathbb{R})$, l'espace des fonctions lisses et à support compact sur $\mathbb{R}$. Soit $I=[a, b]$ un intervalle qui contient le support de $f$. Les fonctions $f^{\prime}$ et $f^{\prime \prime}$ sont aussi lisses et les supports de $f^{\prime}$ et $f^{\prime \prime}$ sont tous contenus dans $I$. Par conséquent, $f^{\prime}$ et $f^{\prime \prime}$ sont des fonctions bornées. Soient $C=\left\|f^{\prime}\right\|_{\text {sup }}$ et $C^{\prime}=\left\|f^{\prime \prime}\right\|_{\text {sup }} / 2$. Soit $h$ la fonction

$$
h(x)= \begin{cases}C^{\prime}(b-a)(2 x-a-b)+C(x-b), & x \leqslant a, \\ -C^{\prime}(b-x)^{2}+C(x-b), & a<x \leqslant b, \\ 0, & x>b .\end{cases}
$$

C'est une fonction croissante concave $\left(2 C^{\prime}(b-a)+C\right)$-lipschitzienne et bornée supérieurement par 0. Donc $h \in G$. D'autre part, $h+f$ est aussi une fonction croissante concave car, sur $[a, b], h^{\prime} \geqslant C$ et $h^{\prime \prime}=-2 C^{\prime}$. Elle est de plus $\left(2 C^{\prime}(b-a)+2 C\right)$ -

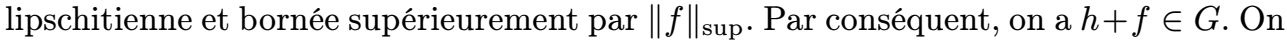
en déduit $f \in G$. Enfin, comme $C_{0}^{\infty}(\mathbb{R})$ est dense dans l'espace normé $\left(C_{c}(\mathbb{R}),\|\cdot\|_{\text {sup }}\right)$, on a $C_{c}(\mathbb{R}) \subset G$, où $C_{c}(\mathbb{R})$ est l'espace des fonctions continues à support compact sur $\mathbb{R}$.

Soit $S: C_{c}(\mathbb{R}) \rightarrow \mathbb{R}$ l'opérateur qui associe à chaque fonction continue à support compact $g$ la limite de la suite $\left(\int_{\mathbb{R}} g \mathrm{~d} \nu_{n}\right)_{n \geqslant 1}$. C'est un opérateur linéaire. De plus, si $g$ est une fonction positive, alors $\int_{\mathbb{R}} g \mathrm{~d} \nu_{n} \geqslant 0$ quel que soit $n \in \mathbb{N}$. Par conséquent, $S(g) \geqslant 0$. D'après le théorème de représentation de Riesz, il existe une unique mesure borélienne finie $\nu$ sur $\mathbb{R}$ telle que $S(g)=\int_{\mathbb{R}} g \mathrm{~d} \nu$. Par définition la suite de mesure $\left(\nu_{n}\right)_{n \geqslant 1}$ converge vaguement vers $\nu$.

REMARQUe 3.3.7. - La limite des mesures obtenue dans la proposition 3.3.6 n'est pas nécessairement une mesure de probabilité : il suffit de considérer le cas où $B=k[X]$ et $\lambda_{\mathscr{F}^{(n)}}\left(X^{n}\right)=n^{2}$. La limite des mesures $T_{\frac{1}{n}} \nu_{\mathcal{F}^{(n)}}=\delta_{n}$ est alors nulle. Cependant, 
si on rajoute une condition supplémentaire que $\lambda_{\max }\left(B_{n}, \mathcal{F}^{(n)}\right)=O(n)(n \rightarrow+\infty)$, alors cette mesure limite est effectivement une mesure de probabilité. En effet, dans ce cas-là les suites $\left(\lambda_{\max }\left(B_{n}, \mathcal{F}^{(n)}\right) / n\right)_{n \geqslant 1}$ et $\left(\lambda_{\min }\left(B_{n}, \mathcal{F}^{(n)}\right) / n\right)_{n \geqslant 1}$ convergent dans $\mathbb{R}$ (voir les propositions 3.2 .4 et 3.2.6), donc les supports des mesures $T_{\frac{1}{n}} \nu_{\mathcal{F}^{(n)}}$ sont uniformément bornés. La proposition 1.2.9 montre donc la mesure limite est une mesure de probabilité.

3.3.3. Variante pseudo-filtrée. - Les résultats que l'on a obtenus dans le sousparagraphe précédent admettent des analogues dans le cas où l'algèbre $B$ est $f$-pseudofiltrée. On présente au-dessous un analogue de la proposition 3.3.6.

Proposition 3.3.8. - Soit $f: \mathbb{N} \rightarrow \mathbb{R}_{\geqslant 0}$ une fonction croissante telle que $\sum_{\alpha \geqslant 0} f\left(2^{\alpha}\right) / 2^{\alpha}<+\infty$. Soit $B=k\left[X_{1}, \ldots, X_{d}\right]$ l'algèbre des polynômes qui est usuellement graduée. Si, pour chaque entier $n \geqslant 1, B_{n}$ est muni d'une $\mathbb{R}$-filtration $\mathcal{F}^{(n)}$ de sorte que $B$ devient une algèbre graduée $f$-pseudo-filtrée, alors la suite de mesures $\left(T_{\frac{1}{n}} \nu_{\mathcal{G}^{(n)}}\right)_{n \geqslant 1}$ converge vaguement. Si de plus $\lambda_{\max }\left(B_{n}, \mathcal{F}^{(n)}\right)=O(n)$, alors la mesure limite est une mesure de probabilité.

\subsection{Convergence des mesures : cas général}

Dans cette section, on démontre le théorème de convergence général par la réduction au cas d'algèbres de polynômes. Une technique classique à attaquer ce genre de problème est de généraliser le problèmes aux modules gradués et puis faire appel à la méthode de dévissage, qui est un argument de récurrence noethérienne. Nous avons déjà utilisé cette technique dans $§ 3.1$ pour les algèbres bigraduées. Pourtant, comme on montrera plus loin dans la remarque 3.4.4, la convergence vague des mesures n'est pas nécessairement vraie pour un "module gradué quasi-filtré". En effet, la condition d'être quasi-filtré(e) donne seulement une minoration de la position (i.e. la valeur de la fonction $\lambda$ ) du produit de plusieurs éléments. C'est la structure d'algèbre qui empêche le produit d'aller trop loin dans la filtration.

On fixe dans cette section une algèbre graduée $B=\bigoplus_{n \geqslant 0} B_{n}$ de type fini sur $k$. On rappelle que, si $M=\bigoplus_{n \in \mathbb{Z}} M_{n}$ est un $B$-module gradué de type fini et non-nul, alors on a l'estimation suivante :

$$
\operatorname{rg}_{k}\left(M_{n}\right)=\frac{c(M)}{(r-1) !} n^{r-1}+o\left(n^{r-1}\right),
$$

où $r$ est la dimension de $M$ et $c(M)$ est une constante qui ne dépend que de $M$. Si $M$ est nul, alors par convention $\operatorname{dim}(M)=-\infty$ et $c(M)=0$. Les énoncés 1 ) et 2) du lemme 3.1.4 sont vrais pour toute suite exacte courte de $B$-modules de type fini. On suppose que l'espace $B_{n}$ est muni d'une $\mathbb{R}$-filtration $\mathcal{F}^{(n)}$. Dans toute la suite de section, si $M$ est un $B$-module gradué de type fini, on suppose, pour tout entier $n \geqslant 1$, que l'espace $M_{n}$ est muni d'une $\mathbb{R}$-filtration que l'on ne précise pas. On utilise les conventions présentées dans $§ 1.2 .6$. Par exemple, l'expression $\nu_{M_{n}}$ désigne la mesure associée à la filtration sous-entendue de $M_{n}$. 


\subsubsection{Condition de convergence vague}

DÉfinition 3.4.1. - Soit $M$ un $B$-module gradué de type fini (muni de $\mathbb{R}$-filtrations). On dit que $M$ satisfait à la condition de convergence vague et on note $\mathbf{C V}(M)$ si la suite de mesures boréliennes $\left(T_{\frac{1}{n}} \nu_{M_{n}}\right)_{n \geqslant 1}$ converge vaguement.

D'après la définition, un $B$-module gradué $M$ de dimension 0 satisfait automatiquement à la condition de convergence vague : $\nu_{M_{n}}=0$ pour $n$ suffisamment grand.

On présente au-dessous un lemme qui étudie la condition de convergence vague pour une suite exacte. Ce lemme sera utilisé dans la démonstration du théorème 3.4.3.

LEMME 3.4.2. - Soit $0 \longrightarrow M^{\prime} \stackrel{\phi}{\longrightarrow} M \stackrel{\pi}{\longrightarrow} M^{\prime \prime} \longrightarrow 0$ une suite exacte courte de B-modules gradués de type fini munis des filtrations. On suppose que, pour tout $n \in \mathbb{Z}, 0 \longrightarrow M_{n}^{\prime} \stackrel{\phi_{n}}{\longrightarrow} M_{n} \stackrel{\pi_{n}}{\longrightarrow} M_{n}^{\prime \prime} \longrightarrow 0$ est une suite exacte d'espaces filtrés. On note $d^{\prime}=\operatorname{dim} M^{\prime}, \quad d=\operatorname{dim} M, \quad d^{\prime \prime}=\operatorname{dim} M^{\prime \prime}$.

1) Si $d^{\prime}>d^{\prime \prime}$, alors $\mathbf{C V}\left(M^{\prime}\right) \Longleftrightarrow \mathbf{C V}(M)$.

2) Si $d^{\prime \prime}>d^{\prime}$, alors $\mathbf{C V}\left(M^{\prime \prime}\right) \Longleftrightarrow \mathbf{C V}(M)$.

3) Si $d^{\prime}=d^{\prime \prime}$, alors $\mathbf{C V}\left(M^{\prime}\right)$ et $\mathbf{C V}\left(M^{\prime \prime}\right) \Longrightarrow \mathbf{C V}(M)$.

Démonstration. - Si $\operatorname{dim} M^{\prime}=0$, alors pour $n$ assez grand, on a $M_{n}=M_{n}^{\prime \prime}$, donc $\mathbf{C V}\left(M^{\prime \prime}\right) \Longleftrightarrow \mathbf{C V}(M)$. Donc le lemme est vraie lorsque $\operatorname{dim} M^{\prime}=0$. On peut démontrer de façon similaire que le lemme est vraie lorsque $\operatorname{dim} M^{\prime \prime}=0$. Dans tout le reste de la démonstration, on supposera $\min \left(d^{\prime}, d^{\prime \prime}\right) \geqslant 1$. On a $d=\max \left(d^{\prime}, d^{\prime \prime}\right)$. Pour tout $n \in \mathbb{N}$, on note

$$
\begin{gathered}
\nu_{n}^{\prime}=T_{\frac{1}{n}} \nu_{M_{n}^{\prime}}, \quad \nu_{n}=T_{\frac{1}{n}} \nu_{M_{n}}, \quad \nu_{n}^{\prime \prime}=T_{\frac{1}{n}} \nu_{M_{n}^{\prime \prime}} \\
r_{n}^{\prime}=\operatorname{rg} M_{n}^{\prime}, \quad r_{n}=\operatorname{rg} M_{n}, \quad r_{n}^{\prime \prime}=\operatorname{rg} M_{n}^{\prime \prime} .
\end{gathered}
$$

Pour $n$ suffisamment grand, $r_{n}^{\prime}, r_{n}$ et $r_{n}^{\prime \prime}$ sont strictement positifs et les mesures $\nu_{n}^{\prime}$, $\nu_{n}$ et $\nu_{n}^{\prime \prime}$ sont des mesures de probabilité. En outre, la proposition 1.2.5 montre que

$$
\nu_{n}=\frac{r_{n}^{\prime}}{r_{n}} \nu_{n}^{\prime}+\frac{r_{n}^{\prime \prime}}{r_{n}} \nu_{n}^{\prime \prime}
$$

D'après (58),

$$
r_{n}^{\prime}=\frac{c\left(M^{\prime}\right)}{\left(d^{\prime}-1\right) !} n^{d^{\prime}-1}+o\left(n^{d^{\prime}-1}\right), \quad r_{n}^{\prime \prime}=\frac{c\left(M^{\prime \prime}\right)}{\left(d^{\prime \prime}-1\right) !} n^{d^{\prime \prime}-1}+o\left(n^{d^{\prime \prime}-1}\right), \quad r_{n}=r_{n}^{\prime}+r_{n}^{\prime \prime} .
$$

1) Si $d^{\prime}>d^{\prime \prime}$, alors

$$
\lim _{n \rightarrow+\infty} \frac{r_{n}^{\prime}}{r_{n}}=1, \quad \lim _{n \rightarrow+\infty} \frac{r_{n}^{\prime \prime}}{r_{n}}=0
$$

donc $\left(\nu_{n}\right)_{n \geqslant 1}$ converge vaguement si et seulement si $\left(\nu_{n}^{\prime}\right)_{n \geqslant 1}$ converge vaguement, et si c'est le cas, elles ont la même limite.

2) Si $d^{\prime \prime}>d^{\prime}$, alors

$$
\lim _{n \rightarrow+\infty} \frac{r_{n}^{\prime}}{r_{n}}=0, \quad \lim _{n \rightarrow+\infty} \frac{r_{n}^{\prime \prime}}{r_{n}}=1
$$


donc $\left(\nu_{n}\right)_{n \geqslant 1}$ converge vaguement si et seulement si $\left(\nu_{n}^{\prime \prime}\right)_{\geqslant 1}$ converge vaguement, et si c'est le cas, elles ont la même limite.

3) Si $d^{\prime \prime}=d^{\prime}$, alors $c(M)=c\left(M^{\prime}\right)+c\left(M^{\prime \prime}\right)$, et

$$
\lim _{n \rightarrow+\infty} \frac{r_{n}^{\prime}}{r_{n}}=\frac{c\left(M^{\prime}\right)}{c(M)}, \quad \lim _{n \rightarrow+\infty} \frac{r_{n}^{\prime \prime}}{r_{n}}=\frac{c\left(M^{\prime \prime}\right)}{c(M)},
$$

Si $\left(\nu_{n}^{\prime}\right)_{n \geqslant 1}$ converge vaguement vers $\nu^{\prime}$ et si $\left(\nu_{n}^{\prime \prime}\right)_{n \geqslant 1}$ converge vaguement vers $\nu^{\prime \prime}$, alors $\left(\nu_{n}\right)_{n \geqslant 1}$ converge vaguement vers $\frac{c\left(M^{\prime}\right)}{c(M)} \nu^{\prime}+\frac{c\left(M^{\prime \prime}\right)}{c(M)} \nu^{\prime \prime}$.

3.4.2. Théorème de convergence des mesures. - Dans ce sous-paragraphe, on établit le théorème principal de l'article qui affirme la convergence des mesures (dilatées) associées à $B$. On en déduit ensuite la convergence uniforme des polygones associés.

ThÉorÈme 3.4.3. - Soit $f: \mathbb{Z}_{\geqslant 0} \rightarrow \mathbb{R}_{\geqslant 0}$ une fonction telle que $\lim _{n \rightarrow+\infty} f(n) / n=0$.

On suppose que

1) l'algèbre graduée $B$ est intègre et $f$-quasi-filtrée, et $B_{n} \neq 0$ pour $n$ assez grand,

2) il existe $\alpha>0$ tel que $\lambda_{\max }\left(B_{n}, \mathcal{F}^{(n)}\right) \leqslant \alpha n$ quel que soit $n \geqslant 1$.

Pour tout entier $n \geqslant 1$, soit $\nu_{n}=T_{\frac{1}{n}} \nu_{\mathcal{F}^{(n)}}$. Alors les supports des mesures $\nu_{n}$ sont uniformément bornés et la suite de mesures $\left(\nu_{n}\right)_{\geqslant 1}$ converge vaguement vers une mesure de probabilité borélienne sur $\mathbb{R}$.

Démonstration. - Soit $n_{0}$ comme dans la définition 3.2.1. Quitte à augmenter la valeur de $n_{0}$ on peut supposer que $B_{n} \neq 0$ lorsque $n \geqslant n_{0}$. D'après [25] II.2.1.6, il existe deux entiers $m_{0}>n_{0}$ et $d_{0}>0$ tels que, pour tout entier $n \geqslant m_{0}$, on ait $B_{d_{0}+n}=B_{d_{0}} B_{n}$. Soit $d=d_{0} m_{0}$. L'algèbre graduée $B^{(d)}=\bigoplus_{n \geqslant 0} B_{n d}$ est engendrée comme une $B_{0^{-}}$ algèbre par $B_{1}^{(d)}=B_{d}$. De plus, si on désigne par $g: \mathbb{N} \rightarrow \mathbb{R}_{\geqslant 0}$ la fonction telle que $g(n)=f(n d)$, alors l'algèbre graduée $B^{(d)}$ est $g$-quasi-filtrée. Pour tout entier $n$ assez grand, on a $B_{n} \neq 0$ et donc $\lambda_{\min }\left(B_{n}, \mathcal{F}^{(n)}\right) \leqslant \lambda_{\max }\left(B_{n}, \mathcal{F}^{(n)}\right) \leqslant \alpha n$. D'après la proposition 3.2.6, la suite $\left(\lambda_{\min }\left(B_{n d}, \mathcal{F}^{(n d)}\right) / n d\right)_{n \geqslant 1}$ converge dans $\mathbb{R}$. De plus, pour tout entier $l \in\left[m_{0}, m_{0}+d\left[\right.\right.$ et tout entier $n \geqslant 1$, on a $B_{n d+l}=B_{n d} B_{l}$. On en déduit (voir la démonstration de la proposition 3.2.6)

$$
\lambda_{\min }\left(B_{n d+l}, \mathcal{F}^{(n d+l)}\right) \geqslant \lambda_{\min }\left(B_{n d}, \mathcal{F}^{(n d)}\right)+\lambda_{\min }\left(B_{l}, \mathcal{F}^{(l)}\right)-f(n d)-f(l) .
$$

Par passage à la limite, on obtient

$$
\liminf _{n \rightarrow+\infty} \lambda_{\min }\left(B_{n d+l}, \mathcal{F}^{(n d+l)}\right) /(n d+l) \geqslant \lim _{n \rightarrow+\infty} \lambda_{\min }\left(B_{n d}, \mathcal{F}^{(n d)}\right) / n d
$$

Comme $l$ est arbitraire, on en déduit que $\liminf _{n \rightarrow+\infty} \lambda_{\min }\left(B_{n}, \mathcal{F}^{(n)}\right) / n$ existe dans $\mathbb{R}$. Si $B_{n} \neq 0$, alors le support de $\nu_{n}$ est contenu dans $\left[\lambda_{\min }\left(B_{n}, \mathcal{F}^{(n)}\right) / n, \lambda_{\max }\left(B_{n}, \mathcal{F}^{(n)}\right) / n\right]$, donc les supports des $\nu_{n}$ sont uniformément bornés.

D'après la proposition 1.2.9, pour démontrer la deuxième assertion du théorème, il suffit d'établir $\mathbf{C V}(B)$. On commence par quelques réductions. 
D'abord, par extension des scalaires (voir §1.2.3), en introduisant une extension infinie de $k$, on peut supposer que $k$ est un corps infini. De plus, il est anodin de supposer $B_{0}=k$, muni de la filtration triviale qui contient un seul saut en 0 .

Si $c$ est une constante réelle, on peut considérer la filtration $\mathcal{F}^{(n), c}$ sur $B_{n}$ telle que $\mathcal{F}_{t}^{(n), c} B_{n}=\mathcal{F}_{t-c n}^{(n)} B_{n}$. Autrement dit, pour tout élément $a \in B_{n}$, on a la relation $\lambda_{\mathcal{F}^{(n), c}}(a)=\lambda_{\mathcal{F}^{(n)}}(a)+c n$. Si $\left(n_{i}\right)_{1 \leqslant i \leqslant r} \in \mathbb{Z}_{\geqslant n_{0}}^{r}$ est un multi-indice et si pour tout $i$, $a_{i}$ est un élément dans $B_{n_{i}}$, en posant $N=n_{1}+\cdots+n_{r}$ et $a=a_{1} \cdots a_{r}$, on a

$$
\begin{aligned}
\lambda_{\mathcal{F}^{(N)}, c}(a) & =\lambda_{\mathcal{F}^{(N)}}(a)+c N \geqslant \sum_{i=1}^{r}\left(\lambda_{\mathcal{F}^{\left(n_{i}\right)}}\left(a_{i}\right)-f\left(n_{i}\right)\right)+\sum_{i=1}^{r} c n_{i} \\
& =\sum_{i=1}^{r}\left(\lambda_{\mathcal{F}^{\left(n_{i}\right), c}}\left(a_{i}\right)-f\left(n_{i}\right)\right),
\end{aligned}
$$

autrement dit, l'algèbre graduée $B$, munie des filtrations $\mathcal{F}^{(n), c}$, est encore $f$-quasifiltrée. D'autre part, si on désigne par $\nu_{B_{n}}^{c}$ la probabilité associée à la filtration $\mathcal{F}^{(n), c}$, on a $\nu_{B_{n}}^{c}=\tau_{c n} \nu_{B_{n}}$, où $\nu_{B_{n}}$ est la mesure associée à $\mathcal{F}^{(n)}$. Par conséquent, on a $T_{\frac{1}{n}} \nu_{B_{n}}^{c}=T_{\frac{1}{n}} \tau_{c n} \nu_{B_{n}}=\tau_{c} T_{\frac{1}{n}} \nu_{B_{n}}$. Autrement dit, $B$ satisfait à la condition de convergence vague pour les filtrations $\mathcal{F}^{(n)}$ si et seulement si c'est le cas pour les filtrations $\mathcal{F}^{(n), c}$. En remplaçant les filtrations $\mathcal{F}^{(n)}$ par $\mathcal{F}^{(n), c}$ avec $c$ suffisamment grand, on se ramène au cas où $\lambda_{\min }\left(B_{n}, \mathcal{F}^{(n)}\right)-f(n) \geqslant 0$ pour tout $n \geqslant 1$. En particulier, pour tout élément homogène $a$ de degré $n$ de $B, \lambda_{\mathscr{F}^{(n)}}(a) \geqslant f(n)$.

La démonstration du théorème se décompose en trois étapes. Sauf dans la dernière étape, on s'impose d'une condition supplémentaire que l'algèbre $B$ est engendrée comme $K$-algèbre par $B_{1}$ et que la constante $n_{0}$ est nulle.

Étape 1: Comme $k$ est un corps infini, par la normalisation de Noether (cf. [19] Théorème 13.3), il existe $d$ éléments $x_{1}, \ldots, x_{d}$ dans $B_{1}$ tels que

1) l'homomorphisme de l'algèbre de polynômes $k\left[T_{1}, \ldots, T_{d}\right]$ vers $B$ qui envoie $T_{i}$ en $x_{i}$ soit un isomorphisme de $k$-algèbres graduées de $k\left[T_{1}, \ldots, T_{d}\right]$ sur son image.

2) si on désigne par $A$ cette image, c'est-à-dire la sous- $k$-algèbre de $B$ engendrée par $x_{1}, \ldots, x_{d}$, alors $B$ soit un $A$-module gradué de type fini.

L'algèbre $A$, munie des filtrations induites, est une $k$-algèbre graduée $f$-quasi-filtrée. La proposition 3.3.6 montre que l'on a $\mathbf{C V}(A)$.

Soit $a$ un élément homogène de $A$. Alors $A a$ est un sous- $A$-module gradué de $B$. On munit $A a$ des filtrations induites (de celles de $B$ ). Comme $\operatorname{dim}(A / A a)<\operatorname{dim} A$, on a CV $(A a)$ compte tenu du lemme 3.4.2. De plus, les suites de mesures de probabilité $\left(T_{\frac{1}{n}} \nu_{A_{n}}\right)_{n \geqslant 1}$ et $\left(T_{\frac{1}{n}} \nu_{(A a)_{n}}\right)_{n \geqslant 1}$ convergent vaguement vers la même limite.

Si $x$ est un élément homogène de degré $m>0$ dans $B \backslash A$, alors il existe un polynôme unitaire $P \in A[X]$ de degré $p \geqslant 2$ tel que $P(x)=0$. On suppose que $P$ est de degré minimal et s'écrit sous la forme $P(X)=X^{p}+a_{p-1} X^{p-1}+\cdots+a_{0}$. 
Comme $P$ est minimal et comme $B$ est un anneau intègre, $a_{0}$ est non-nul. Pour tout $i \in\{0, \ldots, p-1\}$, soit $\widetilde{a}_{i}$ la composante de degré $(p-i) m$ de $a_{i}$. Si on note

$$
\widetilde{P}(X)=X^{p}+\widetilde{a}_{p-1} X^{p-1}+\cdots+\widetilde{a}_{0},
$$

alors on a $\widetilde{P}(x)=0$ puisque $x$ est homogène de degré $m$. On peut donc supposer que $a_{i}$ est homogène de degré $(p-i) m$ quel que soit $i \in\{0, \ldots, p-1\}$. Soit $y=$ $x^{p-1}+a_{p-1} x^{p-2}+\cdots+a_{1}$. Il est homogène de degré $(p-1) m$. En outre, on a $x y+a_{0}=0$. Dans la suite, on utilise les notations simplifiées introduites dans $\S 1.2 .6$ pour les fonctions $\lambda$. Puisque l'algèbre graduée $B$ est $f$-quasi-filtrée, si $u$ est un élément homogène de degré $n$ de $A$, alors

$$
\lambda\left(u a_{0}\right)=\lambda(u x y) \geqslant \lambda(u x)-f(n+m)+\lambda(y)-f((p-1) m) \geqslant \lambda(u x)-f(n+m),
$$

où dans la dernière inégalité, on a utilisé l'hypothèse

$$
\lambda_{\min }\left(B_{(p-1) m}, \mathcal{F}^{(p-1) m}\right) \geqslant f((p-1) m)
$$

introduite dans l'étape de réduction. On en déduit $\lambda(u x) \leqslant \lambda\left(u a_{0}\right)+f(n+m)$. En outre,

$$
\lambda(u x) \geqslant \lambda(u)+\lambda(x)-f(m)-f(n) \geqslant \lambda(u)-f(n) .
$$

Soient $M=A a_{0}, M^{\prime}=A x$. L'algèbre $B$ étant intègre, pour tout entier $n \geqslant 1$, l'application $u x \mapsto u a_{0}\left(u \in A_{n}\right)$ est un isomorphisme de $k$-espaces vectoriels de $M_{n+m}^{\prime}$ vers $M_{n+m p}$. D'après (59) et le lemme 1.2.6, on a $\nu_{M_{n+m}^{\prime}} \prec \tau_{f(n+m)} \nu_{M_{n+m p}}$. D'autre part, l'application $u \mapsto u x\left(u \in A_{n}\right)$ est un isomorphisme de $k$-espaces vectoriels de $A_{n}$ vers $M_{n+m}^{\prime}$. D'après (60) et le lemme 1.2.6, on obtient $\nu_{A_{n}} \prec \tau_{f(n)} \nu_{M_{n+m}^{\prime}}$, ou encore $\tau_{-f(n)} \nu_{A_{n}} \prec \nu_{M_{n+m}^{\prime}}$. On obtient donc l'encadrement

$$
\tau_{-f(n)} \nu_{A_{n}} \prec \nu_{M_{n+m}^{\prime}} \prec \tau_{f(n+m)} \nu_{M_{n+m p}},
$$

et donc

ou encore

$$
T_{\frac{1}{n+m}} \tau_{-f(n)} \nu_{A_{n}} \prec T_{\frac{1}{n+m}} \nu_{M_{n+m}^{\prime}} \prec T_{\frac{1}{n+m}} \tau_{f(n+m)} \nu_{M_{n+m p}}
$$

$$
\tau_{\frac{-f(n)}{n+m}} T_{\frac{n}{n+m}} T_{\frac{1}{n}} \nu_{A_{n}} \prec T_{\frac{1}{n+m}} \nu_{M_{n+m}^{\prime}} \prec \tau_{\frac{f(n+m)}{n+m}} T_{\frac{n+m p}{n+m}} T_{\frac{1}{n+m p}} \nu_{M_{n+m p}} .
$$

Comme observé plus haut, les suites $\left(T_{\frac{1}{n}} \nu_{A_{n}}\right)_{n \geqslant 1}$ et $\left(T_{\frac{1}{n}} \nu_{M_{n}}\right)_{n \geqslant 1}$ convergent vaguement vers une même limite que l'on note $\nu$. D'après le lemme 1.2.11, l'encadrement (61), et le lemme 1.2.12, on conclut que la suite $\left(T_{\frac{1}{n}} \nu_{M_{n}^{\prime}}\right)_{n \geqslant 1}$ converge vaguement aussi vers $\nu$.

Étape 2: Soit $k^{\prime}$ le corps des fractions de $A$. Comme $B$ est une algèbre finie sur $A$, l'algèbre $k^{\prime} \otimes_{A} B$ est de rang fini sur $k^{\prime}$. Le $A$-module $B$ est engendré par les éléments homogènes, il existe donc des éléments homogènes $x_{1}, \ldots, x_{s}$ de $B$ qui forment une base de $k^{\prime} \otimes_{A} B$ sur $k^{\prime}$. Si on note $H=A x_{1}+\cdots+A x_{s}$, alors $H$ est un sous- $A$-module libre de base $\left(x_{1}, \ldots, x_{s}\right)$ de $B$. Soit $H^{\prime}=B / H$. On a une suite exacte :

$$
0 \longrightarrow H \stackrel{\psi}{\longrightarrow} B \stackrel{\pi}{\longrightarrow} H^{\prime} \longrightarrow 0 \text {. }
$$


Comme $1 \otimes \psi: k^{\prime} \otimes_{A} H \rightarrow k^{\prime} \otimes_{A} B$ est un isomorphisme, on a $k^{\prime} \otimes_{A} H^{\prime}=0$, donc $H^{\prime}$ est un $A$-module de torsion. On a alors $\operatorname{dim}_{A} H^{\prime}<\operatorname{dim} A=\operatorname{dim}_{A} H=\operatorname{dim}_{A} B$. D'après l'étape 2 , la condition $\mathbf{C V}\left(A x_{i}\right)$ est vérifiée pour tout $1 \leqslant i \leqslant s$. D'après le lemme 3.4.2, on a $\mathbf{C V}(H)$ et puis $\mathbf{C V}(B)$. Le théorème est donc démontré pour le cas particulier où $B$ est engendrée comme une $B_{0}$-algèbre par $B_{1}$, et où $n_{0}=0$.

Étape 3: On traite maintenant le cas général. Comme remarqué au début de la démonstration, il existe un entier $d>n_{0}$ tel que $B^{(d)}=\bigoplus_{n \geqslant 0} B_{d n}$ soit une $B_{0^{-}}$ algèbre engendrée par $B_{1}^{(d)}=B_{d}$. C'est un anneau intègre. De plus, elle est $g$-quasifiltrée pour la fonction $g(n)=f(n d)$, où cette fois-ci la constante " $n_{0}$ " pour cette algèbre est nulle. D'après le résultat déjà démontré, on a $\mathbf{C V}\left(B^{(d)}\right)$. On désigne par $\rho$ la limite de $\left(T_{\frac{1}{n d}} \nu_{B_{n d}}\right)_{n \geqslant 1}$. Soit $x$ un élément homogène de degré $l \geqslant n_{0}$ de $B$. On suppose $x \neq 0$. On a $x^{d} \in B_{l d}$. Si $u$ est un élément dans $B_{n d}$, alors

$$
\begin{aligned}
\lambda\left(u x^{d}\right) & \geqslant \lambda\left(x^{d-1}\right)-f(l d-l)+\lambda(u x)-f(n d+l) \\
& \geqslant \lambda(u x)-f(n d+l),
\end{aligned}
$$

où on a utilisé l'estimation $\lambda\left(x^{d-1}\right) \geqslant f(l d-l)$ convenue dans l'étape de réduction. De façon similaire, on a

$$
\lambda(u x) \geqslant \lambda(u)-f(n d)+\lambda(x)-f(l) \geqslant \lambda(u)-f(n d) .
$$

D'où

$$
\tau_{f(n d+l)} \nu_{B_{n d+l d}} \succ \nu_{B_{n d} x} \succ \tau_{-f(n d)} \nu_{B_{n d}} .
$$

Par passage à la limite, en s'appuyant sur le lemme 1.2.11, on obtient que la suite de mesures $\left(T_{\frac{1}{n d}} \nu_{B_{n d} x}\right)_{n \geqslant 1}$ converge vaguement vers $\rho$. D'autre part, pour tout entier $k$ tel que $n_{0} \stackrel{\text { nd }}{\leqslant} k<n_{0}+d$, l'espace $B^{(d, k)}=\bigoplus_{n \geqslant 0} B_{n d+k}$ est non-nul. C'est un $B^{(d)}$ module de type fini. Soient $k^{(d)}$ le corps de fractions de $B^{(d)}$ et $\left(x_{i}\right)_{i=1}^{s_{d, k}}$ une famille d'éléments homogène dans $B^{(d, k)}$ qui forme une base de $B^{(d, k)} \otimes_{B^{(d)}} k^{(d)}$ sur $k^{(d)}$. Soit $H^{(d, k)}=\bigoplus_{i=1}^{s_{d, k}} B^{(d)} x_{i}$. C'est un sous- $B^{(d)}$-module libre de $B^{(d, k)}$ tel que

$$
\operatorname{dim}_{B^{(d)}}\left(B^{(d, k)} / H^{(d, k)}\right)<\operatorname{dim}_{B^{(d)}}\left(B^{(d, k)}\right) .
$$

D'après ce que l'on a démontré, le lemme 3.4.2 montre que la suite $\left(T_{\frac{1}{n d}} \nu_{H^{(d, k)}}\right)_{n \geqslant 1}$ converge vaguement vers $\rho$. Encore par le lemme 3.4.2, joint au fait que

$$
\operatorname{dim}_{B^{(d)}}\left(B^{(d, k)} / H^{(d, k)}\right)<\operatorname{dim}_{B^{(d)}}\left(B^{(d, k)}\right),
$$

on conclut que $B^{(d, k)}$ satisfait à la condition de convergence vague, et que la limite de la suite $\left(T_{\frac{1}{n d}} \nu_{B_{n d+k}}\right)_{n \geqslant 1}$ coïncide avec $\rho$. Enfin, en combinant ces suites de probabilités, on conclut que la suite de probabilités $\left(T_{\frac{1}{n}} \nu_{B_{n}}\right)_{n \geqslant 1}$ converge vaguement vers $\rho$.

REmARQue 3.4.4. - 1) La condition d'être quasi-filtré peut s'étendre facilement au cas de module gradué. Soit $f: \mathbb{N} \rightarrow \mathbb{R}_{\geqslant 0}$ une application. On suppose que l'algèbre graduée $B$ est $f$-quasi-filtrée. Soit $M$ un $B$-module gradué muni des filtrations 
$\left(\mathscr{G}^{(n)}\right)_{n \geqslant 1}$. On dit que $M$ est $f$-quasi-filtré s'il existe un entier $n_{0} \geqslant 0$ tel que, pour tout entier $r>0$, tout $\left(n_{i}\right)_{1 \leqslant i \leqslant r+1} \in \mathbb{Z}_{\geqslant n_{0}}^{r+1}$ et tout $\left(s_{i}\right)_{1 \leqslant i \leqslant r+1} \in \mathbb{R}^{r+1}$, on ait

$\left(\prod_{i=1}^{r} \mathcal{F}_{s_{i}}^{\left(n_{i}\right)} B_{n_{i}}\right) \mathscr{G}_{s_{r+1}}^{\left(n_{r+1}\right)} M_{n_{r+1}} \subset \mathscr{G}_{S}^{(N)} M_{N}, \quad$ où $\quad N=\sum_{i=1}^{r+1} n_{i}$ et $S=\sum_{i=1}^{r+1}\left(s_{i}-f\left(n_{i}\right)\right)$.

Si $f=0$ et si $n_{0}=0, M$ est dit filtré. Cependant, l'assertion du théorème 3.4.3 n'est pas vraie en général pour un module gradué (quasi-)filtré. En effet, soit $B$ l'algèbre $k[X]$ des polynômes à une variable munie de la graduation usuelle et de la filtration $\mathcal{F}^{(n)}$ telle que

$$
\mathcal{F}_{t}^{(n)} B_{n}= \begin{cases}B_{n}, & \text { si } t \leqslant 0 \\ 0, & \text { si } t>0\end{cases}
$$

Evidemment $B$ est une $k$-algèbre graduée filtrée. Soit $M$ le $B$-module gradué libre engendré par un élément homogène de degré 0 . Soit $\varphi: \mathbb{Z}_{\geqslant 0} \rightarrow \mathbb{R}$ une fonction croissante. Pour tout entier $n \geqslant 1$, on définit une filtration $\mathscr{G}^{\varphi,(n)}$ sur $M_{n}$ telle que

$$
\mathscr{G}_{t}^{\varphi,(n)} M_{n}= \begin{cases}M_{n}, & \text { si } t \leqslant \varphi(n), \\ 0, & \text { si } t>\varphi(n) .\end{cases}
$$

Alors $M$ est un $B$-module gradué filtré et, pour tout entier $n \geqslant 0, \nu_{M_{n}}=\delta_{\varphi(n)}$. La condition $\mathbf{C V}(M)$ est équivalente à l'existence de $\lim _{n \rightarrow+\infty} \varphi(n) / n$ dans $\mathbb{R} \cup\{+\infty\}$. Si $\varphi: \mathbb{Z}_{\geqslant 0} \rightarrow \mathbb{R}$ est une fonction croissante telle que la suite $(\varphi(n) / n)_{n \geqslant 1}$ ait plusieurs points adhérents - par exemple, $\varphi(n)=2^{\left\lfloor\log _{2} n\right\rfloor}, \mathbf{C V}(M)$ n'est plus satisfaite. Ce contre-exemple montre l'impossibilité de démontrer le théorème 3.4 .3 par la version classique de la technique de dévissage.

2) L'assertion du théorème 3.4.3 n'est pas vraie en général pour une algèbre graduée quasi-filtrée non-intègre. Soient $B$ et $M$ comme dans 1) plus haut. Si on désigne par $C$ l'extension nilpotente de $B$ par $M$ (cf. [35] chap. $9 \S 25$ ), alors $C$ est une algèbre graduée quasi-filtrée sur $K$, mais la condition $\mathbf{C V}(C)$ n'est pas vraie. La même construction fournit aussi un contre-exemple de la proposition 3.2.4 lorsque l'algèbre $B$ n'est pas intègre.

Corollaire 3.4.5. - Avec les mêmes hypothèses du théorème 3.4.3, la suite de polygones $\left(\mathscr{P}\left(\nu_{n}\right)\right)_{n \geqslant 1}$ converge uniformément vers une fonction concave sur $[0,1]$.

Démonstration. - C'est une conséquence du théorème 3.4.3 et de la proposition 1.2.9.

3.4.3. Variante pseudo-filtrée. - On présente enfin la version pseudo-filtrée du théorème 3.4.3.

ThÉORÈme 3.4.6. - Soit $f: \mathbb{Z}_{\geqslant 0} \rightarrow \mathbb{R}_{\geqslant 0}$ une fonction croissante telle que $\sum_{\alpha \geqslant 0} f\left(2^{\alpha}\right) / 2^{\alpha}<+\infty$. On suppose que

1) l'algèbre graduée $B$ est intègre et $f$-pseudo-filtrée, et $B_{n} \neq 0$ pour $n$ assez grand, 
2) il existe $\alpha>0$ tel que $\lambda_{\max }\left(B_{n}, \mathcal{F}^{(n)}\right) \leqslant \alpha n$ quel que soit $n \geqslant 1$.

Pour tout entier $n \geqslant 1$, soit $\nu_{n}=T_{\frac{1}{n}} \nu_{\mathcal{F}^{(n)}}$. Alors les supports des mesures $\nu_{n}$ sont uniformément bornés, la suite de mesures $\left(\nu_{n}\right)_{\geqslant 1}$ converge vaguement vers une mesure de probabilité borélienne sur $\mathbb{R}$, et la suite de polygones $\left(\mathscr{P}\left(\nu_{n}\right)\right)_{n \geqslant 1}$ converge uniformément sur $[0,1]$. 


\section{CHAPITRE 4}

\section{APPLICATIONS}

\subsection{Théorème de Hilbert-Samuel arithmétique}

Le théorème de Hilbert-Samuel arithmétique étudie le comportement asymptotique des caractéristiques d'Euler-Poincaré des images directes des puissances tensorielles d'un fibré inversible hermitien sur une variété arithmétique. Ce théorème était d'abord démontré par Gillet et Soulé [23] en utilisant leur théorème de Riemann-Roch arithmétique. Puis il a été réétudié par plusieurs auteurs comme Abbes et Bouche [1], Zhang [45], Rumely, Lau et Varley [41], Autissier [2] et Randriambololona [38] dans divers contextes. Dans le cas où les métriques sur le fibré inversible hermitien sont positives et le fibré inversible sous-jacent est ample, ce comportement asymptotique peut être interprété par le nombre d'intersection du fibré inversible hermitien considéré.

Dans cette section, on applique la convergence de polygones à l'étude du théorème de Hilbert-Samuel. Comme le résultat précédemment établi est très général, on obtient le théorème de Hilbert-Samuel (la partie de convergence) dans toute généralité sans condition de positivité sur les métriques.

4.1.1. Comparaison des limites. - Étant donnée une suite de fibrés vectoriels adéliques, on montrera que, si on modifie convenablement les métriques de chaque fibré vectoriel adélique de la suite, le comportement asymptotique (convergence ou divergence) reste inchangé.

Proposition 4.1.1. - Soient

$$
\left(\bar{B}_{n}=\left(B_{n},\left(\|\cdot\|_{v}\right)_{v \in \Sigma}\right)\right)_{n \geqslant 1} \quad \text { et } \quad\left(\bar{B}_{n}^{\prime}=\left(B_{n},\left(\|\cdot\|_{v}^{\prime}\right)_{v \in \Sigma}\right)\right)_{n \geqslant 1}
$$

deux suites de fibrés vectoriels adéliques sur $\operatorname{Spec} K$ qui ont la même suite d'espaces vectoriels sous-jacents $\left(B_{n}\right)_{n \geqslant 1}$ telle que $\lim _{n \rightarrow \infty} \log \left(\operatorname{rg}\left(B_{n}\right)\right) / n=0$. On suppose que,

(i) pour tout entier $n \geqslant 1$ et toute place finie $\mathfrak{p} \in \Sigma_{f}$, les métriques $\|\cdot\|_{\mathfrak{p}}$ et $\|\cdot\|_{\mathfrak{p}}^{\prime}$ sur $B_{n} \otimes_{K} \mathbb{C}_{\mathfrak{p}}$ sont la même, 
(ii) on a la relation

$$
\max _{v \in \Sigma_{\infty}} \sup _{0 \neq s \in B_{n} \otimes_{K} \mathbb{C}_{v}}\left|\log \|s\|_{v}-\log \|s\|_{v}^{\prime}\right|=o(n) \quad(n \rightarrow \infty) .
$$

Alors les propiétés suivantes sont vérifiées.

1) La suite $\left(\widehat{\mu}_{\max }\left(\bar{B}_{n}\right) / n\right)_{n \geqslant 1} \quad$ (resp. $\left.\left(\widehat{\mu}_{\min }\left(\bar{B}_{n}\right) / n\right)_{n \geqslant 1}\right)$ converge dans $\mathbb{R}$ si et seulement si la suite $\left(\widehat{\mu}_{\max }\left(\bar{B}_{n}^{\prime}\right) / n\right)_{n \geqslant 1}$ (resp. $\left.\left(\widehat{\mu}_{\min }\left(\bar{B}_{n}^{\prime}\right) / n\right)_{n \geqslant 1}\right)$ converge dans $\mathbb{R}$. De plus, dans le cas où ces deux suites sont convergentes, elles ont la même limite.

2) La suite $\left(\mathscr{P}_{\bar{B}_{n}} / n\right)_{n \geqslant 1}$ converge (resp. converge uniformément) si et seulement si la suite $\left(\mathscr{P}_{\bar{B}_{n}^{\prime}} / n\right)_{n \geqslant 1}$ converge (resp. converge uniformément). De plus, si ces deux suites convergent, alors elles convergent vers la même limite.

Démonstration. - Pour tout entier $n \geqslant 1$, on note

$$
\alpha_{n}=\max _{v \in \Sigma_{\infty}} \sup _{0 \neq s \in B_{n} \otimes_{K} \mathbb{C}_{v}}\left|\log \|s\|_{v}-\log \|s\|_{v}^{\prime}\right| .
$$

D'après les propositions 2.1.6 et 2.1.7, on a

$$
\left|\widehat{\mu}_{\max }\left(\bar{B}_{n}\right)-\widehat{\mu}_{\max }\left(\bar{B}_{n}^{\prime}\right)\right| \leqslant \alpha_{n} \quad \text { et } \quad\left|\widehat{\mu}_{\min }\left(\bar{B}_{n}\right)-\widehat{\mu}_{\min }\left(\bar{B}_{n}^{\prime}\right)\right| \leqslant \alpha_{n} .
$$

Comme $\lim _{n \rightarrow \infty} \alpha_{n} / n=0$, l'assertion 1) est vraie pour $\left(\bar{B}_{n}\right)_{n \geqslant 1}$ et $\left(\bar{B}_{n}^{\prime}\right)_{n \geqslant 1}$.

Pour démontrer l'assertion 2), on commence par un cas particulier où les $\bar{B}_{n}$ et $\bar{B}_{n}^{\prime}$ sont hermitiens. Dans ce cas-là, on a $\left\|\mathscr{P}_{\bar{B}_{n}}-\mathscr{P}_{\bar{B}_{n}^{\prime}}\right\|_{\text {sup }} \leqslant \alpha_{n}$, compte tenu du corollaire 2.2.9. Donc l'assertion 2) est vraie pour ce cas particulier.

Pour démontrer le cas général, on introduit quelques notations. On désigne par $\mathbb{P}\left(\left(\bar{B}_{n}\right)_{n \geqslant 1},\left(\bar{B}_{n}^{\prime}\right)_{n \geqslant 1}\right)$ la condition de la proposition et par $\mathbb{Q}\left(\left(\bar{B}_{n}\right)_{n \geqslant 1},\left(\bar{B}_{n}^{\prime}\right)_{n \geqslant 1}\right)$ l'assertion 2). On observe que $\mathbb{P}$ et $\mathbb{Q}$ sont des relations d'équivalence sur l'ensemble des suites en fibrés vectoriels adéliques. Soit $\mathbf{E}:=\left(\bar{E}_{n}\right)_{n \geqslant 1}$ une suite de fibrés vectoriels adéliques. Soient $\mathbf{E}_{\mathrm{J}}:=\left(\bar{E}_{n, \mathrm{~J}}\right)_{n \geqslant 1}$ et $\mathbf{E}_{\mathrm{L}}:=\left(\bar{E}_{n, \mathrm{~L}}\right)_{n \geqslant 1}$ (voir $\S 2.1 .5$ pour les notations). Par définition, la condition $\mathbb{P}\left(\mathbf{E}_{\mathrm{J}}, \mathbf{E}_{\mathrm{L}}\right)$ est vérifiée. D'après le cas particulier que l'on a démontré, l'assertion $\mathbb{Q}\left(\mathbf{E}_{\mathrm{J}}, \mathbf{E}_{\mathrm{L}}\right)$ est vraie. En outre, pour tout fibré vectoriel adélique $\bar{E} \operatorname{sur} \operatorname{Spec} K$, on a des encadrements $\mathscr{P}_{\bar{E}_{\mathrm{J}}} \leqslant \mathscr{P}_{\bar{E}} \leqslant \mathscr{P}_{\bar{E}_{\mathrm{L}}}$. C'est une conséquence de la proposition 2.1.5 (voir aussi la remarque 2.2.10). Par conséquent, l'assertion $\mathbb{Q}\left(\mathbf{E}_{\mathrm{J}}, \mathbf{E}\right)$ est vraie pour toute suite $\mathbf{E}$ en fibrés vectoriels adéliques. Maintenant si $\mathbf{B}$ et $\mathbf{B}^{\prime}$ sont deux suites en fibrés vectoriels adéliques qui vérifient $\mathbb{P}\left(\mathbf{B}, \mathbf{B}^{\prime}\right)$, alors la condition $\mathbb{P}\left(\mathbf{B}_{\mathrm{J}}, \mathbf{B}_{\mathrm{J}}^{\prime}\right)$ est encore vérifiée. On en déduit $\mathbb{Q}\left(\mathbf{B}_{\mathrm{J}}, \mathbf{B}_{\mathrm{J}}^{\prime}\right)$, compte tenu de ce que l'on a démontré plus haut. En outre, les assertions $\mathbb{Q}\left(\mathbf{B}_{\mathrm{J}}, \mathbf{B}\right)$ et $\mathbb{Q}\left(\mathbf{B}_{\mathrm{J}}^{\prime}, \mathbf{B}^{\prime}\right)$ sont vraies. Comme $\mathbb{Q}$ est une relation d'équivalence, on obtient $\mathbb{Q}\left(\mathbf{B}, \mathbf{B}^{\prime}\right)$. 
4.1.2. Algèbre en fibrés vectoriels adéliques. - Soient $K$ un corps de nombres et $B=\bigoplus_{n \geqslant 0} B_{n}$ une algèbre intègre sur $K$ telle que chaque $B_{n}$ est un espace vectoriel de rang fini sur $K$ et que $B_{n} \neq 0$ pour $n$ suffisamment grand. On suppose que $B_{n}$ est l'espace vectoriel sous-jacent d'un fibré vectoriel adélique $\bar{B}_{n}$ sur Spec $K$. Pour tout entier $r \geqslant 2$ et tout élément $\mathbf{n}=\left(n_{i}\right)_{1 \leqslant i \leqslant r} \in \mathbb{N}^{r}$, on désigne par $\psi_{\mathbf{n}}$ : $B_{n_{1}} \otimes \cdots \otimes B_{n_{r}} \rightarrow B_{|\mathbf{n}|}$ l'application $K$-linéaire induite par la structure de $K$-algèbre de $B$, où $|\mathbf{n}|=n_{1}+\cdots+n_{r}$. Le théorème suivant étudie le comportement asymptotique des invariants arithmétiques des $\bar{B}_{n}$.

ThÉORÈme 4.1.2. - Soit $B=\bigoplus_{n \geqslant 0} B_{n}$ une algèbre intègre sur $K$ telle que $B_{n} \neq 0$ pour $n$ assez grand et que $B_{n}$ soit l'espace vectoriel sous-jacent d'un fibré vectoriel adélique $\bar{B}_{n}$. On suppose qu'il existe $C>0$ tel que $\widehat{\mu}_{\max }\left(\bar{B}_{n}\right) \leqslant C n$ pour $n \geqslant 1$, et que l'une des deux conditions suivantes est vérifiée :

(i) $\lim _{n \rightarrow+\infty} \log \left(\operatorname{rg} B_{n}\right) / n=0$ (cette condition est satisfaite notamment lorsque $B$ est une algèbre de type fini), et il existe une fonction $g: \mathbb{N} \rightarrow \mathbb{R}_{\geqslant 0}$ et un entier $n_{0} \geqslant 0$ tels que $\lim _{n \rightarrow+\infty} g(n) / n=0$ et que la hauteur de

$$
\psi_{\mathbf{n}}: \bar{B}_{n_{1}} \otimes_{\alpha} \cdots \otimes_{\alpha} \bar{B}_{n_{r}} \longrightarrow \bar{B}_{|\mathbf{n}|}
$$

est majorée par $g\left(n_{1}\right)+\cdots+g\left(n_{r}\right)$ pour tous $r \in \mathbb{Z}_{\geqslant 2}, \mathbf{n}=\left(n_{i}\right) \in \mathbb{Z}_{\geqslant n_{0}}^{r}$ et pour une famille hermitienne $\alpha$ de normes tensorielles d'ordre $r$,

(ii) $\log \left(\operatorname{rg} B_{n}\right)=O(\log n)$, et il existe une fonction croissante $g: \mathbb{N} \rightarrow \mathbb{R}_{\geqslant 0}$ et un entier $n_{0} \geqslant 0$ tels que $\sum_{u \geqslant 0} g\left(2^{u}\right) / 2^{u}<+\infty$ et que $h\left(\psi_{(n, m)}\right) \leqslant g(n)+g(m)$ pour tous les entiers $n$ et $m$ supérieurs ou égaux à $n_{0}$, et pour une famille hermitienne de normes tensorielles d'ordre 2.

Alors la suite de pentes maximales $\left(\widehat{\mu}_{\max }\left(\bar{B}_{n}\right) / n\right)_{n \geqslant 1}$ converge dans $\mathbb{R}$. Si de plus l'algèbre $B$ est de type fini, alors

1) la suite de polygones de Harder-Narasimhan $\left(\mathscr{P}_{\bar{B}_{n}} / n\right)_{n \geqslant 1}$ converge uniformément vers une fonction concave sur $[0,1]$;

2) les suites $\left(\widehat{\mu}\left(\bar{B}_{n}\right) / n\right)_{n \geqslant 1}$ et $\left(d ! \chi\left(\bar{B}_{n}\right) / n^{d}\right)_{n \geqslant 1}$ convergent dans $\mathbb{R}$, où $d$ est la dimension de Krull de B.

Enfin, si l'homomorphisme $\psi_{(n, m)}$ est surjectif pour $n$ et $m$ assez grand, alors la suite de pentes minimales $\left(\widehat{\mu}_{\min }\left(\bar{B}_{n}\right) / n\right)_{n \geqslant 1}$ converge dans $\mathbb{R}$.

Démonstration. - On commence par le cas particulier où les fibrés vectoriels adéliques $\bar{B}_{n}$ sont hermitiens. Pour tout entier $n \geqslant 1$, on désigne par $\mathcal{F}^{(n)}$ la filtration de Harder-Narasimhan de $\bar{B}_{n}$.

On suppose la condition (i) et on démontre que l'algèbre graduée $B$ est $f$-quasifiltrée pour $f(n)=g(n)+\log \left(\operatorname{rg} B_{n}\right)$. En effet, si $\left(s_{i}\right)_{1 \leqslant i \leqslant r}$ est un élément dans $\mathbb{R}^{r}$, alors on a l'inégalité

$$
\widehat{\mu}_{\min }\left(\overline{\mathcal{F}}_{s_{1}}^{\left(n_{1}\right)} B_{n_{1}} \otimes \cdots \otimes \overline{\mathcal{F}}_{s_{r}}^{\left(n_{r}\right)} B_{n_{r}}\right) \geqslant \sum_{i=1}^{r}\left(\widehat{\mu}_{\min }\left(\overline{\mathcal{F}}_{s_{i}}^{\left(n_{i}\right)} B_{n_{i}}\right)-\log \left(\operatorname{rg} B_{n_{i}}\right)\right)
$$


compte tenu de (30). D'après la proposition 2.2.1, on obtient

$$
\widehat{\mu}_{\min }\left(\overline{\mathcal{F}}_{s_{1}}^{\left(n_{1}\right)} B_{n_{1}} \otimes \cdots \otimes \overline{\mathcal{F}}_{s_{r}}^{\left(n_{r}\right)} B_{n_{r}}\right) \geqslant \sum_{i=1}^{r}\left(s_{i}-\log \left(\operatorname{rg} B_{n_{i}}\right)\right)
$$

Par conséquent, $\psi_{\mathbf{n}}\left(\mathcal{F}_{s_{1}}^{\left(n_{1}\right)} B_{n_{1}} \otimes \cdots \otimes \mathcal{F}_{s_{r}}^{\left(n_{r}\right)} B_{n_{r}}\right)$ est contenu dans $\mathcal{F}_{t}^{(|\mathbf{n}|)} B_{|\mathbf{n}|}$ où $t=$ $\sum_{i=1}^{r}\left(s_{i}-\log \left(\operatorname{rg} B_{n_{i}}\right)\right)-h\left(\psi_{|\mathbf{n}|}\right)$ (cf. proposition 2.2.4 infra). Cela montre que $B$ est $f$-quasi-filtrée. Comme $\lim _{n \rightarrow \infty} \log \left(\operatorname{rg} B_{n}\right) / n=0$, on a $\lim _{n \rightarrow+\infty} f(n) / n=0$. D'après la proposition 3.2.4, on obtient la convergence de $\left(\widehat{\mu}_{\max }\left(\bar{B}_{n}\right) / n\right)_{n \geqslant 1}$. Si l'algèbre $B$ est de type fini, alors le corollaire 3.4 .5 montre que les polygones $\mathscr{P}\left(T_{\frac{1}{n}} \nu_{\bar{B}_{n}}\right)=\mathscr{P}_{\bar{B}_{n}} / n$ convergent uniformément quand $n \rightarrow+\infty$. Dans le cas où $\psi_{(n, m)}$ est surjectif lorsque $n$ et $m$ sont assez grands, la convergence de $\left(\widehat{\mu}_{\min }\left(\bar{B}_{n}\right) / n\right)_{n \geqslant 1}$ résulte de la proposition 3.2.6.

Lorsque l'algèbre $B$ est de type fini, la convergence de $\left(\widehat{\mu}\left(\bar{B}_{n}\right) / n\right)_{n \geqslant 1}$ provient de la convergence uniforme des polygones $\mathscr{P}_{\bar{B}_{n}} / n$ et l'égalité $\widehat{\mu}(\bar{E})=\mathscr{P}_{\bar{E}}(1)$ qui est valable pour tout fibré vectoriel adélique non-nul $\bar{E}$. En outre, d'après la remarque 2.1.4, il existe une constante $b$ telle que $\left|\chi\left(\bar{K}^{m}\right)\right| \leqslant b m(\log m+1)$ pour tout entier $m \geqslant 1$. On en déduit donc $\lim _{n \rightarrow+\infty} \chi\left(\bar{K}^{\mathrm{rg} B_{n}}\right) /\left(n \mathrm{rg} B_{n}\right)=0$. D'après la relation entre le degré d'Arakelov et la caractéristique d'Euler-Poincaré (16), on obtient que

$$
\lim _{n \rightarrow+\infty} \chi\left(\bar{B}_{n}\right) /\left(n \operatorname{rg} B_{n}\right)=\lim _{n \rightarrow+\infty}[K: \mathbb{Q}] \widehat{\mu}\left(\bar{B}_{n}\right) / n
$$

existe dans $\mathbb{R}$. Enfin, comme $B$ est de dimension de Krull $d$, il existe une constante $c(B)>0$ tel que $\operatorname{rg}\left(B_{n}\right) \sim c(B) n^{d-1}$, d'où la convergence de $\left(\chi\left(\bar{B}_{n}\right) /\left(n \operatorname{rg} B_{n}\right)\right)_{n \geqslant 1}$ implique celle de $\left(d ! \chi\left(\bar{B}_{n}\right) / n^{d}\right)_{n \geqslant 1}$.

La démonstration du cas particulier sous la condition (ii) est très similaire à celle qui précède, en utilisant la variante pseudo-filtrée. En effet, la fonction $\log \left(\operatorname{rg} B_{n}\right)$ est de croissance logarithmique. Comme $\sum_{u \geqslant 0} \log \left(2^{u}\right) / 2^{u}<+\infty$, on obtient la proposition en s'appuyant sur le théorème 3.4.6 et les propositions 3.2.9 et 3.2.10.

La démonstration du cas général repose sur les résultats de comparaisons dans les propositions 2.1.15 et 4.1.1. En effet, si les fibrés vectoriels adéliques $\bar{B}_{n}$ satisfont à $\widehat{\mu}_{\max }\left(\bar{B}_{n}\right)=O(n)$ et l'une des conditions (i) et (ii), alors il en est de même des fibrés adéliques hermitiens $\bar{B}_{n, \mathrm{~J}}$ : d'abord, d'après (33), on a $\widehat{\mu}_{\max }\left(\bar{B}_{n, \mathrm{~J}}\right) \leqslant \widehat{\mu}_{\max }\left(\bar{B}_{n}\right)$; en outre, d'après la proposition 2.1.15, quitte à augmenter la fonction $g$ par un terme logarithmique, on obtient que la condition (i) (resp. (ii)) est vérifiée pour les $\bar{B}_{n}$ si et seulement si elle est vérifiée pour les $\bar{B}_{n, \mathrm{~J}}$. Compte tenu de ce qu'on a démontré plus haut, le théorème est vrai pour les fibrés adéliques hermitiens $\bar{B}_{n, \mathrm{~J}}$, et donc le même résultat est vrai pour les $\bar{B}_{n}$, grâce à la proposition 4.1.1.

Remarque 4.1.3. - On garde les notations du théorème 4.1.2. Pour tout entier $n \geqslant$ 1 , on choisit un fibré adélique hermitien $\bar{B}_{n}^{\prime}=\left(B_{n},\left(\|\cdot\|_{v}^{\prime}\right)_{v \in \Sigma}\right)$ tel que, en écrivant 
$\bar{B}_{n}=\left(B_{n},\left(\|\cdot\|_{v}\right)_{v \in \Sigma}\right)$, on ait $\|\cdot\|_{\mathfrak{p}}=\|\cdot\|_{\mathfrak{p}}^{\prime}$ pour tout $\mathfrak{p} \in \Sigma_{f}$, et

$$
\max _{v \in \Sigma_{\infty}} \sup _{0 \neq s \in B_{n} \otimes_{K} \mathbb{C}_{v}}\left|\log \|s\|_{v}-\log \|s\|_{v}^{\prime}\right|=o(n) \quad(n \rightarrow \infty) .
$$

D'après (33), on obtient que $\widehat{\mu}_{\max }\left(\bar{B}_{n}\right)=O(n)$ si et seulement si $\widehat{\mu}_{\max }\left(\bar{B}_{n}^{\prime}\right)=O(n)$. En particulier, $\widehat{\mu}_{\max }\left(\bar{B}_{n}\right)=O(n)$ si et seulement si $\widehat{\mu}_{\max }\left(\bar{B}_{n, \mathrm{~J}}\right)=O(n)$, ou encore si et seulement si $\widehat{\mu}_{\max }\left(\bar{B}_{n, \mathrm{~L}}\right)=O(n)$.

En outre, d'après la proposition 2.1.15, la condition (i) du théorème 4.1.2 équivaut à

$\left(\mathrm{i}^{\prime}\right) \lim _{n \rightarrow \infty} \log \left(\operatorname{rg} B_{n}\right) / n=0$, et il existe une fonction $g: \mathbb{N} \rightarrow \mathbb{R}_{\geqslant 0}$ et un entier $n_{0} \geqslant 0$ tels que $\lim _{n \rightarrow+\infty} g(n) / n=0$ et que la hauteur de $\psi_{\mathbf{n}}: \bigotimes_{i=1}^{r} \bar{B}_{n_{i}}^{\prime} \longrightarrow \bar{B}_{|\mathbf{n}|}^{\prime}$ est majorée par $g\left(n_{1}\right)+\cdots+g\left(n_{r}\right)$ pour tout $r \in \mathbb{Z}_{\geqslant 2}$ et tout $\mathbf{n}=\left(n_{i}\right) \in \mathbb{Z}_{\geqslant n_{0}}^{r}$.

En particulier, elle est équivalente à chacune des conditions suivantes:

(i $\left.i_{1}\right) \lim _{n \rightarrow \infty} \log \left(\operatorname{rg} B_{n}\right) / n=0$, et il existe une fonction $g: \mathbb{N} \rightarrow \mathbb{R}_{\geqslant 0}$ et un entier $n_{0} \geqslant 0$ tels que $\lim _{n \rightarrow+\infty} g(n) / n=0$ et que la hauteur de $\psi_{\mathbf{n}}: \bigotimes_{i=1}^{r} \bar{B}_{n_{i}, \mathrm{~J}} \longrightarrow \bar{B}_{|\mathbf{n}|, \mathrm{J}}$ est majorée par $g\left(n_{1}\right)+\cdots+g\left(n_{r}\right)$ pour tout $r \in \mathbb{Z}_{\geqslant 2}$ et tout $\mathbf{n}=\left(n_{i}\right) \in \mathbb{Z}_{\geqslant n_{0}}^{r}$.

(i $\left.\mathrm{i}_{2}\right) \lim _{n \rightarrow \infty} \log \left(\operatorname{rg} B_{n}\right) / n=0$, et il existe une fonction $g: \mathbb{N} \rightarrow \mathbb{R}_{\geqslant 0}$ et un entier $n_{0} \geqslant 0$ tels que $\lim _{n \rightarrow+\infty} g(n) / n=0$ et que la hauteur de $\psi_{\mathbf{n}}: \bigotimes_{i=1}^{r} \bar{B}_{n_{i}, \mathrm{~L}} \longrightarrow \bar{B}_{|\mathbf{n}|, \mathrm{L}}$ est majorée par $g\left(n_{1}\right)+\cdots+g\left(n_{r}\right)$ pour tout $r \in \mathbb{Z}_{\geqslant 2}$ et tout $\mathbf{n}=\left(n_{i}\right) \in \mathbb{Z}_{\geqslant n_{0}}^{r}$.

De façon similaire, on a des conditions équivalentes à la condition (ii) du théorème 4.1.2. Ces observations - combinées avec la proposition 4.1.1 - permettent de se ramener au cas de fibrés adéliques hermitiens dans l'étude du comportement asymptotique des invariants arithmétiques, comme ce qu'on a fait dans la démonstration du théorème 4.1.2.

Si $B_{n} \neq 0$ pour $n$ suffisamment grand, si l'algèbre $B=\bigoplus_{n \geqslant 0} B_{n}$ est de type fini, $\widehat{\mu}_{\text {max }}\left(\bar{B}_{n}\right)=O(n)$ et si les fibrés vectoriels adéliques $\bar{B}_{n}$ vérifient les conditions dans (i) du théorème 4.1.2, alors l'algèbre graduée $B$ est $f$-quasi-filtrée pour les filtrations de Harder-Narasimhan des $\bar{B}_{n}^{\prime}$ relativement à une fonction $f: \mathbb{N} \rightarrow \mathbb{R}_{+}$telle que $\lim _{n \rightarrow \infty} f(n) / n=0$. D'après le théorème 3.4 .3 , la suite de mesures $\left(T_{\frac{1}{n}} \nu_{\bar{B}_{n}^{\prime}}^{\prime}\right)_{n \geqslant 1}$ converge vaguement vers une mesure de probabilité $\nu$ sur $\mathbb{R}$, et cette mesure limite ne dépend pas du choix des métriques sur les $\bar{B}_{n}^{\prime}$. La limite des polygones $\mathscr{P}_{\bar{B}_{n}} / n$ coïncide avec $\mathscr{P}(\nu)$.

De façon similaire, si $B_{n} \neq 0$ pour $n$ suffisamment grand, si l'algèbre $B=\bigoplus_{n \geqslant 0} B_{n}$ est de type fini, $\widehat{\mu}_{\max }\left(\bar{B}_{n}\right)=O(n)$ et si les fibrés vectoriels adéliques $\bar{B}_{n}$ vérifient conditions (ii) du théorème 4.1.2, alors l'algèbre graduée $B$ est $f$-pseudo-filtrée pour les filtrations de Harder-Narasimhan des $\bar{B}_{n}^{\prime}$ relativement à une fonction $f: \mathbb{N} \rightarrow \mathbb{R}_{+}$ telle que $\sum_{u \geqslant 0} f\left(2^{u}\right) / 2^{u}<+\infty$. On déduit du théorème 3.4.6 que la suite de mesures $\left(T_{\frac{1}{n}} \nu_{\bar{B}_{n}^{\prime}}^{\prime}\right)_{n \geqslant 1}$ converge vaguement vers une mesure de probabilité $\nu$ sur $\mathbb{R}$ et que la limite des polygones $\mathscr{P}_{\bar{B}_{n}} / n$ coïncide avec $\mathscr{P}(\nu)$. 
Enfin, on remarque que les invariants asymptotiques considérés dans le théorème 4.1.2 sont les mêmes (une fois s'ils existent) pour les $\bar{B}_{n}$ et pour les $\bar{B}_{n}^{\prime}$.

4.1.3. Existence de la capacité sectionnelle. - Le théorème 4.1.2 peut être comparé avec le théorème $(\mathrm{A})$ de [41]. Soient $X$ un schéma intègre projectif de dimension $d$ définie sur $K$ et $L$ un fibré inversible sur $X$. Si chaque espace de sections globales $\Gamma\left(X, L^{\otimes n}\right)$ est muni des normes de sorte que $\overline{\Gamma\left(X, L^{\otimes n}\right)}$ soit un fibré vectoriel adélique, on dit que $\bar{L}$ est un fibré inversible avec sections normées. La capacité sectionnelle de $\bar{L}$ est par définition

$$
S_{\gamma}(\bar{L})=\exp \left(-\lim _{n \rightarrow+\infty} \frac{(d+1) !}{n^{d+1}} \chi\left(\overline{\Gamma\left(X, L^{\otimes n}\right)}\right)\right)
$$

pourvu que la limite dans le terme à droite existe dans $\mathbb{R} \cup\{+\infty\}$. Cette notion est d'abord introduite par Chinburg [18]. Elle généralise deux notions en même temps : la capacité logarithmique d'un diviseur ample dans $X$ et le nombre d'autointersection d'un fibré inversible hermitien arithmétiquement ample. L'existence de capacité sectionnelle est étudiée par plusieurs auteurs dans une série d'articles comme $[\mathbf{1 8}, \mathbf{3 9}, \mathbf{4 0}, \mathbf{4 1}$. En particulier, cette existence est démontrée dans [41] à une grande généralité avec eventuellement des semi-normes sur $\Gamma\left(X, L^{\otimes n}\right)$.

Le théorème 4.1.2 donne un critère d'existence de la capacité sectionnelle.

Corollaire 4.1.4. - On suppose que l'algèbre $\bigoplus_{n \geqslant 0} \Gamma\left(X, L^{\otimes n}\right)$ est de type fini sur $K$ (cette condition est vérifiée notamment lorsque $L$ est ample) et telle que $\Gamma\left(X, L^{\otimes n}\right) \neq 0$ pour $n$ suffisamment grand. Si pour chaque entier $n \geqslant 0$, on munit l'espace $\Gamma\left(X, L^{\otimes n}\right)$ d'une structure de fibré vectoriel adélique de sorte que $\widehat{\mu}_{\max }\left(\overline{\Gamma\left(X, L^{\otimes n}\right)}\right)=O(n)(n \rightarrow \infty)$ et que les fibrés vectoriels adéliques $\overline{\Gamma\left(X, L^{\otimes n}\right)}$ vérifient l'une des conditions (i) et (ii) du théorème 4.1.2 (ou l'une parmi leurs formes équivalentes introduites dans la remarque 4.1.3), alors la capacité sectionnelle $S_{\gamma}(\bar{L})$ existe et est non-nul.

Démonstration. - C'est une conséquence de l'assertion 2) du théorème 4.1.2, sachant que la dimension de Krull de l'algèbre $\bigoplus_{n \geqslant 0} \Gamma\left(X, L^{\otimes n}\right)$ est au plus $d+1$.

Remarque 4.1.5. - Dans [41], les auteurs ont obtenu l'existence de la capacité sectionnelle sous des hypothèses de compatibilité et de finitude pour les métriques. En particulier, l'une des conditions de compatibilité algébrique (A1),

$$
\begin{aligned}
& \|f g\|_{v} \leqslant\|f\|_{v}\|g\|_{v} \text { quels que soient } v \in \Sigma_{f} \cup \Sigma_{\infty}, f \in \mathbb{C}_{v} \otimes \Gamma\left(X, L^{\otimes n}\right) \text { et } \\
& g \in \mathbb{C}_{v} \otimes \Gamma\left(X, L^{\otimes m}\right),
\end{aligned}
$$

implique les conditions (i) et (ii) du théorème 4.1.2. Pour tout entier $n \geqslant 0$, on note $B_{n}=\Gamma\left(X, L^{\otimes n}\right)$. Pour tout entier $n \geqslant 0$ et toute $v \in \Sigma_{\infty}$, on choisit une base

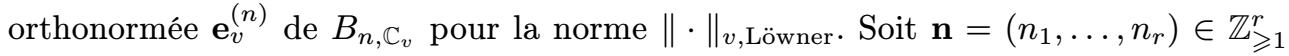
une famille finie d'indices. Alors

$$
\mathbf{e}_{v}^{(\mathbf{n})}:=\left\{s_{1} \otimes \cdots \otimes s_{r} \mid \forall i \in\{1, \ldots, r\}, s_{i} \in \mathbf{e}_{v}^{(i)}\right\}
$$


est une base orthonormée pour la métrique $\|\cdot\|_{v, \mathrm{~L}}$ produit tensoriel des métriques de Löwner. Soit $\psi_{\mathbf{n}, v}: B_{n_{1}, \mathbb{C}_{v}} \otimes \cdots \otimes B_{n_{r}, \mathbb{C}_{v}} \rightarrow B_{|\mathbf{n}|, \mathbb{C}_{v}}$ l'homomorphisme induit par la multiplication des sections. Si $s=s_{1} \otimes \cdots \otimes s_{r}$ est un élément dans $\mathbf{e}_{v}^{(\mathbf{n})}$, alors

$$
\begin{aligned}
& \log \left\|\psi_{\mathbf{n}, v}(s)\right\|_{v, \text { Löwner }} \leqslant \log \left\|\psi_{\mathbf{n}, v}(s)\right\|_{v} \leqslant \sum_{i=1}^{r} \log \left\|s_{i}\right\|_{v} \\
\leqslant & \sum_{i=1}^{r} \log \left\|s_{i}\right\|_{v, \text { Löwner }}+\frac{1}{2} \log \left(\operatorname{rg} B_{n_{i}}\right)=\frac{1}{2} \sum_{i=1}^{r} \log \left(\operatorname{rg} B_{n_{i}}\right)=\frac{1}{2} \log \left(\# \mathbf{e}_{v}^{(\mathbf{n})}\right) .
\end{aligned}
$$

Un élément général $x \in B_{n_{1}, \mathbb{C}_{v}} \otimes \cdots \otimes B_{n_{r}, \mathbb{C}_{v}}$ s'écrit sous la forme $\sum_{s \in \mathbf{e}_{v}^{(\mathbf{n})}} a_{s} s$, où $a_{s} \in \mathbb{C}_{v}$. Par conséquent, on a

$$
\left\|\psi_{\mathbf{n}, v}(x)\right\|_{v, \text { Löwner }} \leqslant \sum_{s \in \mathbf{e}_{v}^{(\mathbf{n})}}\left|a_{s}\right|_{v} \cdot\left\|\psi_{\mathbf{n}, v}(s)\right\|_{v, \text { Löwner }} \leqslant\left(\# \mathbf{e}_{v}^{(\mathbf{n})}\right)^{\frac{1}{2}} \sum_{s \in \mathbf{e}_{v}^{(\mathbf{n})}}\left|a_{s}\right|_{v} .
$$

Par l'inégalité de Cauchy-Schwarz, on obtient $\left\|\psi_{\mathbf{n}, v}(x)\right\|_{v, \text { Löwner }} \leqslant\left(\# \mathbf{e}_{v}^{(\mathbf{n})}\right)\|x\|_{v, \mathrm{~L}}$. Donc les fibrés adéliques hermitiens $\left(\bar{B}_{n, \mathrm{~L}}\right)_{n \geqslant 1}$ vérifie la condition $\left(\mathrm{i}_{2}\right)$ de la remarque 4.1.3.

D'autre part, dans [41], la capacité sectionnelle est justifiée d'être strictement positive pourvu que la condition de croissance suivante dans le théorème $(\mathrm{A}) \mathrm{du}$ loc. cit. est vérifiée :

$$
\text { il existe } \epsilon>0 \text { tel que } \inf _{0 \neq f \in \Gamma(X, L \otimes n)} \prod_{v}\|f\|_{v} \geqslant \epsilon^{n} .
$$

Mais cette condition n'est rien d'autre que la condition $\widehat{\mu}_{\max }\left(\bar{B}_{n}\right)=O(n)$ dans le théorème 4.1.2 compte tenu de la comparaison du premier minimum à la pente maximale, établie dans [5] (voir aussi [22] théorème 5.20). Le point de vue algébrique que l'on a adopté permet d'obtenir le corollaire 4.1.4 pour le cas où $L$ est éventuellement non-ample.

4.1.4. Théorème de Hilbert-Samuel arithmétique. - Soient $\mathscr{X}$ un schéma projectif et plat sur $\operatorname{Spec} \theta_{K}$ et $\mathscr{L}$ un fibré inversible sur $\mathscr{X}$. Soient $X=\mathscr{X}_{K}$, $L:=\mathscr{L}_{K}$ et $d=\operatorname{dim} X$. On suppose que $X$ est intègre et que $E_{n}:=H^{0}\left(X, L^{\otimes n}\right)$ est non-nul lorsque $n$ est suffisamment grand. Pour chaque plongement $\sigma: K \rightarrow \mathbb{C}$, on munit le faisceau $\mathscr{L}_{\sigma, \mathbb{C}}$ d'une métrique hermitienne continue $\|\cdot\|_{\sigma}$. On suppose que les métriques $\|\cdot\|_{\sigma}$ sont invariantes par la conjugaison complexe. Pour tout entier $n \geqslant 0$, soit $\mathcal{E}_{n}=\pi_{*}\left(\mathscr{L}^{\otimes n}\right)$, où $\pi: \mathscr{X} \rightarrow \operatorname{Spec} \theta_{K}$ est le morphisme structurel. Pour tout plongement $\sigma: K \rightarrow \mathbb{C}$, on désigne par $\|\cdot\|_{\sigma, \text { sup }}$ la norme sur $E_{n, \sigma}:=E_{n} \otimes_{K, \sigma} \mathbb{C}=$ $\Gamma\left(X_{\sigma, \mathbb{C}}, L_{\sigma, \mathbb{C}}^{\otimes n}\right)$ telle que $\|s\|_{\sigma, \text { sup }}=\sup _{x \in \mathscr{X}_{\sigma}(\mathbb{C})}\left\|s_{x}\right\|_{\sigma}$. La structure de $\emptyset_{K}$-module sur $\mathcal{E}_{n}$ avec les normes $\|\cdot\|_{\sigma \text {,sup }}$ induisent une structure de fibré vectoriel adélique sur $E_{n}$. On désigne par $\bar{E}_{n}$ le fibré vectoriel adélique correspondant.

Dans [22, théorème 5.20], Gaudron compare les pentes successives d'un fibré vectoriel adélique à ses minima successifs. Cela généralise un résultat de [5] dans le cadre de fibrés vectoriels hermitiens, qui remonte sur le théorème de Minkowski 
adélique dû à Bombieri et Vaaler [3]. On rappelle ici un cas particulier de ce résultat, où on compare la pente maximal au premier minimum. Si $\bar{\delta}=\left(\mathcal{E},\left(\|\cdot\|_{\sigma}\right)_{\sigma: K \rightarrow \mathbb{C}}\right)$ un fibré vectoriel normé non-nul sur $\operatorname{Spec} \theta_{K}$ et si $i \in\{1,2, \ldots, \mathrm{rg} E\}$, on désigne par

$$
\lambda_{i}(\overline{\mathcal{E}}):=\inf \left\{a>0 \mid \operatorname{rg}\left(\operatorname{Vect}_{K}\{\mathbb{B}(\overline{\mathcal{E}}, a)\}\right) \geqslant i\right\}
$$

le $i^{\text {ème }}$ minimum de $\bar{E}$, où

$$
\mathbb{B}(\overline{\mathcal{E}}, a):=\left\{s \in \mathcal{E} \mid \forall \sigma,\|s\|_{\sigma} \leqslant a\right\} .
$$

En particulier, $\lambda_{1}(\overline{\mathcal{E}})=\inf _{0 \neq s \in \mathcal{E}} \max _{\sigma: K \rightarrow \mathbb{C}}\|s\|_{\sigma}$. Attention : ici on utilise la définition de Bombieri et Vaaler [3] pour les minima successifs; or dans [22], Gaudron a pris le point de vue de Thunder [44].

Proposition 4.1.6. - Soit $\bar{E}$ un fibré vectoriel normé de rang $n>0$ sur $\operatorname{Spec} \theta_{K}$. Les inégalités suivantes sont vérifiées

$$
0 \leqslant \widehat{\mu}_{\max }(\overline{\mathcal{E}})+\log \lambda_{1}(\overline{\mathcal{E}}) \leqslant U(n, K),
$$

ò̀ $U(n, K):=-\frac{1}{n[K: \mathbb{Q}]} \chi\left(\bar{K}^{n}\right)+\log 2+\frac{1}{2} \log n \ll_{K} \log (n+1)$.

Démonstration. - Soit $s$ un élément dans $\mathcal{E}$ tel que $\max _{\sigma: K \rightarrow \mathbb{C}}\|s\|_{\sigma}=\lambda_{1}(\overline{\mathcal{E}})$. L'homomorphisme $\bar{\theta}_{K} \rightarrow \overline{\mathcal{E}}$ induit par $s$ est de hauteur $\leqslant \log \lambda_{1}(\overline{\mathscr{E}})$. Par l'inégalité $(22)$, on obtient $0 \leqslant \widehat{\mu}_{\max }(\overline{\mathcal{E}})+\log \lambda_{1}(\overline{\mathcal{E}})$.

On démontrera la majoration de $\widehat{\mu}_{\max }(\overline{\mathcal{E}})+\log \lambda_{1}(\overline{\mathcal{E}})$. Par définition, si $\overline{\mathcal{F}}$ est un sous-fibré vectoriel normé de $\overline{\mathcal{E}}$, alors $\lambda_{1}(\overline{\mathcal{F}}) \geqslant \lambda_{1}(\overline{\mathcal{E}})$. On est donc ramené au cas où $\bar{\delta}$ est semi-stable, c'est-à-dire $\widehat{\mu}_{\max }(\overline{\mathscr{E}})=\widehat{\mu}(\overline{\mathscr{E}})$. Soit $n$ le rang de $\mathcal{E}$. D'après [36, théorème 5] (voir aussi [3, théorème 3], [4], [22, théorème 5.19]), on a

$$
\sum_{i=1}^{n} \log \lambda_{i}(\overline{\mathscr{E}})+\frac{1}{[K: \mathbb{Q}]} \chi(\overline{\mathscr{E}}) \leqslant n \log 2+n \log \sqrt{n} .
$$

Comme $\lambda_{i}(\overline{\mathscr{E}}) \geqslant \lambda_{1}(\overline{\mathcal{E}})$ pour tout $i \in\{1, \ldots, n\}$, on obtient la majoration souhaitée en s'appuyant sur (16). Enfin, d'après $(17)$, on a $U(n, K) \ll_{K} \log (n+1)$.

On démontre que la suite $\left(\widehat{\mu}_{\max }\left(\bar{E}_{n}\right)\right)_{n \geqslant 1}$ est bornée supérieurement par une suite linéaire. Il suffit de minorer la norme du plus petit vecteur non-nul dans $H^{0}\left(\mathscr{X}, \mathscr{L}^{\otimes n}\right)$. Cela est achevé en utilisant le fait que la hauteur d'un cycle effectif par rapport à un fibré inversible hermitien arithmétiquement ample est positive ou nulle.

On rappelle brièvement la notion d'amplitude arithmétique. Soient $\mathscr{Y}$ un $\theta_{K^{-}}$ schéma projectif et plat dont la fibre générique $\mathscr{Y}_{K}$ est lisse et $\overline{\mathcal{L}}$ un fibré inversible hermitien sur $\mathscr{Y}$ dont les métriques sont lisses. Pour tout $q$-cycle $Z$ de $\mathscr{Y}$, la hauteur (relative à $K$ ) de $Z$ est définie comme le nombre d'intersection arithmétique $h_{\bar{L}}(Z):=$ $\left(\widehat{c}_{1}(\bar{L})^{q} \cdot[Z]\right)$ (voir $[\mathbf{1 0}, \S 3]$ pour le détail). On dit que $\overline{\mathcal{L}}$ est ample si $\mathscr{L}$ est relativement ample, si $c_{1}(\overline{\mathscr{L}})$ est une $(1,1)$-forme strictement positive et si, pour tout sous-schéma fermé irréductible $Z$ de $\mathscr{Y}$ qui est plat sur $\operatorname{Spec} \Theta_{K}$, la hauteur $h_{\overline{\mathscr{L}}}(Z)$ est strictement 
positive. D'après [45, corollaire 4.8] et [10, proposition 3.2.4], si $\overline{\mathcal{L}}$ est ample, alors pour tout cycle effectif $Z$, on a $h_{\bar{L}}(Z) \geqslant 0$.

LEMME 4.1.7. - Il existe une constante $C$ telle que $\widehat{\mu}_{\max }\left(\bar{E}_{n}\right) \leqslant C n$ pour tout entier $n$ suffisamment grand.

Démonstration. - Supposons que $\nu: \mathscr{X}^{\prime} \rightarrow \mathscr{X}$ est un morphisme projectif et birationnel. Alors, pour tout entier $n \geqslant 1$, l'homomorphisme injectif naturel $\Gamma\left(\mathscr{X}, \mathscr{L}^{\otimes n}\right) \rightarrow \Gamma\left(\mathscr{X}^{\prime},\left(\nu^{*} \mathscr{L}\right)^{\otimes n}\right)$ est de hauteur nulle pour les sup-normes, d'où $\widehat{\mu}_{\max }\left(\overline{\Gamma\left(\mathscr{X}, \mathscr{L}^{\otimes n}\right)}\right) \leqslant \widehat{\mu}_{\max }\left(\overline{\Gamma\left(\mathscr{X}^{\prime}, \nu^{*} \mathscr{L}^{\otimes n}\right)}\right)$. Grâce au théorème de résolution de singularités dû à Hironaka, on est ramené au cas où $\mathscr{X}_{K}$ est lisse. D'après le théorème de Weierstrass-Stone, on peut supposer que les métriques sur $\mathscr{L}$ sont lisses.

Quitte à choisir un plongement de $\mathscr{X}$ dans un espace projectif et considérer le tir en arrière du fibré inversible canonique muni des métriques de Fubini-Study, on obtient qu'il existe un fibré inversible hermitien $\overline{\mathscr{L}^{\prime}}$ sur $\mathscr{X}$ qui est ample (cf. [10, théorème 5.2.3]). En particulier, si $s$ est une section non-nulle de $\mathscr{L}^{\otimes n}$ sur $\mathscr{X}$, alors divs est un diviseur effectif de $\mathscr{X}$. Par conséquent, on obtient que

$$
h \overline{\mathscr{L}^{\prime}}(\operatorname{div} s)=\widehat{c}_{1}\left(\overline{\mathscr{L}^{\prime}}\right)^{d} \cdot \widehat{c}_{1}\left(\overline{\mathscr{L}}^{\otimes n}\right)+\int_{\mathscr{X}(\mathbb{C})} \log \|s\| c_{1}\left(\overline{\mathscr{L}^{\prime}}\right)^{d} \geqslant 0,
$$

compte tenu de $[\mathbf{1 0},(3.2 .2)]$, où $\mathscr{X}(\mathbb{C})$ est l'ensemble des points complexes de $\mathscr{X} \otimes_{\mathbb{Z}} \mathbb{C}$, muni de la topologie analytique, qui est une variété analytique complexe. En outre, comme $c_{1}\left(\overline{\mathscr{L}^{\prime}}\right)$ est strictement positive, on obtient

$$
\int_{\mathscr{X}(\mathbb{C})} \log \|s\| c_{1}\left(\overline{\mathscr{L}^{\prime}}\right)^{d} \leqslant \max _{\sigma \in \Sigma_{\infty}} \log \|s\|_{\sigma, \sup } \int_{\mathscr{X}(\mathbb{C})} c_{1}\left(\overline{\mathscr{L}^{\prime}}\right)^{d} .
$$

Si on note

$$
C_{1}=\left(\widehat{c}_{1}\left(\overline{\mathscr{L}^{\prime}}\right)^{d} \cdot \widehat{c}_{1}(\overline{\mathscr{L}})\right) /\left(\int_{\mathscr{X}(\mathbb{C})} c_{1}\left(\overline{\mathscr{L}^{\prime}}\right)^{d}\right)
$$

on obtient $-\max _{\sigma} \log \|s\|_{\sigma \text {,sup }} \leqslant C_{1} n$, qui implique $-\log \lambda_{1}\left(\bar{E}_{n}\right) \leqslant C_{1} n$. D'après (63), on obtient $\widehat{\mu}_{\max }\left(\bar{E}_{n}\right) \leqslant C_{1} n+U\left(\operatorname{rg} E_{n}, K\right)=O(n)$.

Le théorème 4.1 .2 conduit à la convergence de plusieurs invariants arithmétiques associés aux $\bar{E}_{n}$.

ThÉORÈme 4.1.8. - Soient $\pi: \mathscr{X} \rightarrow$ Spec $\theta_{K}$ un morphisme projectif et plat tel que $\mathscr{X}_{K}$ soit intègre de dimension $d$, et $\overline{\mathscr{L}}$ un fibré inversible hermitien sur $\mathscr{X}$. Pour tout entier $n \geqslant 1$, on note $\mathcal{E}_{n}=\pi_{*}\left(\mathscr{L}^{\otimes n}\right)$ et on désigne par $\bar{E}_{n}$ le fibré adélique hermitien défini par $\mathcal{E}_{n}$ et les normes sup. On suppose que $E_{n} \neq 0$ pour $n$ suffisamment grand.

1) La suite $\left(\widehat{\mu}_{\max }\left(\bar{E}_{n}\right) / n\right)_{n \geqslant 1}$ converge dans $\mathbb{R}$. 
2) Si l'algèbre $\bigoplus_{n \geqslant 0} H^{0}\left(\mathscr{X}_{K}, \mathscr{L}_{K}^{\otimes n}\right)$ est de type fini, alors la suite de polygones de Harder-Narasimhan $\left(\mathscr{P}_{\bar{E}_{n}} / n\right)_{n \geqslant 1}$ converge uniformément vers une fonction concave $\mathscr{P} \frac{\pi}{\mathscr{L}}$. En particulier, on a

$$
\lim _{n \rightarrow+\infty} \frac{(d+1) !}{n^{d+1}} \chi\left(\bar{E}_{n}\right)=[K: \mathbb{Q}](d+1) \operatorname{vol}\left(\mathscr{L}_{K}\right) \mathscr{P}^{\frac{\pi}{\mathscr{L}}}(1) .
$$

Si $\mathscr{L}$ est ample et si $c_{1}(\overline{\mathscr{L}})$ est positif en tant que $(1,1)$-courant, alors

$$
\mathscr{P}_{\mathscr{L}}^{\pi}(1)=\frac{\widehat{c}_{1}(\overline{\mathscr{L}})^{d+1}}{[K: \mathbb{Q}](d+1) c_{1}\left(\mathscr{L}_{K}\right)^{d}} .
$$

3) Si l'homomorphisme $E_{n} \otimes E_{m} \rightarrow E_{n+m}$ est surjectif pour tous entiers $n$ et $m$ suffisamment grands, alors la suite $\left(\widehat{\mu}_{\min }\left(\bar{E}_{n}\right) / n\right)_{n \geqslant 1}$ converge dans $\mathbb{R}$.

Démonstration. - La remarque 4.1 .5 et le lemme 4.1 .7 montrent que $\widehat{\mu}_{\max }\left(\bar{E}_{n}\right)=$ $O(n)$ et que les fibrés adéliques hermitiens $\bar{E}_{n}$ satisfont aux conditions (i) et (ii) du théorème 4.1.2, on obtient donc les assertions sauf les égalités (64) et (65). Mais (64) est une conséquence de (62) et de l'égalité

$$
\lim _{n \rightarrow+\infty} \frac{\operatorname{rg} E_{n}}{n^{d} / d !}=\operatorname{vol}\left(\mathscr{L}_{K}\right) .
$$

L'égalité (65) provient de [38, Théorème A].

REMARQUe 4.1.9. - On peut aussi travailler avec les métriques $L^{2}$, en choisissant une mesure de Lebesgue de densité lisse sur $\mathscr{X}(\mathbb{C})$. D'après un résultat de Gromov [23, Lemma 30], la proposition 4.1.1 montre que le théorème 4.1.8 reste valable pour les $E_{n}$ munis des métriques $L^{2}$, et que les limites des invariants arithmétiques sont les mêmes.

4.1.5. Exemples. - Dans ce sous-paragraphe, on calcule explicitement les invariants asymptotiques du faisceau inversible canonique sur un espace projectif, muni des métriques de Fubini-Study.

Puissances symétriques. - Soit $V$ un espace hermitien dont le produit scalaire est $\langle,\rangle_{V}$. Pour tout entier $n \geqslant 1$, on appelle $n^{\text {ième }}$ puissance symétrique de $V$ l'espace vectoriel $S^{n} V$ muni du produit hermitien $\langle,\rangle_{S^{n} V}$ tel que

$$
\left\langle x_{1} \cdots x_{n}, y_{1} \cdots y_{n}\right\rangle_{S^{n} V}=\frac{1}{n !} \sum_{\tau \in \mathfrak{S}_{n}} \prod_{j=1}^{n}\left\langle x_{j}, y_{\tau(j)}\right\rangle_{V},
$$

où $\mathfrak{S}_{n}$ désigne le $n^{\text {ième }}$ groupe symétrique. Si $\left(v_{i}\right)_{1 \leqslant i \leqslant r}$ est une base orthogonale de $V$, alors $\left(v^{I}\right)_{I \in \mathbb{N}^{r},|I|=n}$ est une base orthogonale de $S^{n} V$, où pour tout $I=\left(i_{1}, \ldots, i_{r}\right) \in$ $\mathbb{N}^{r}$, on définit $|I|:=i_{1}+\cdots+i_{r}$ et $v^{I}=v_{1}^{i_{1}} \cdots v_{r}^{i_{r}}$. En outre, la norme de $v^{I}$ est

$$
\left\|v^{I}\right\|=\left(\frac{n !}{i_{1} ! \cdots i_{r} !}\right)^{-\frac{1}{2}}\left\|v_{1}\right\|^{i_{1}} \cdots\left\|v_{r}\right\|^{i_{r}}
$$

Soit $\mathfrak{p}$ un idéal maximal de $\theta_{K}$. On suppose que $W$ est un espace vectoriel de rang fini sur $\mathbb{C}_{\mathfrak{p}}$, muni d'une ultranorme $\|\cdot\|$. Alors l'espace vectoriel $S^{n} W$ est naturellement 
muni d'une ultranorme. On suppose que $\left(w_{i}\right)_{1 \leqslant i \leqslant r}$ est une base $\mathfrak{p}$-adique de $W$. C'est une base de $W$ telle que

$$
\left\|a_{1} w_{1}+\cdots+a_{r} w_{r}\right\|=\max _{1 \leqslant i \leqslant r}\left|a_{i}\right|_{\mathfrak{p}}\left\|w_{i}\right\|
$$

Alors $\left(w^{I}\right)_{I \in \mathbb{N}^{r},|I|=n}$ est une base $\mathfrak{p}$-adique de $W$. En outre, on a

$$
\left\|w^{I}\right\|=\left\|w_{1}\right\|^{i_{1}} \cdots\left\|w_{r}\right\|^{i_{r}} \text {, où } I=\left(i_{1}, \ldots, i_{r}\right) .
$$

Considérons maintenant un fibré adélique hermitien $\bar{E} \operatorname{sur} \operatorname{Spec} K$, qui est une somme directe de fibrés inversibles hermitiens $\left(\bar{L}_{i}\right)_{i=1}^{r}$. Dans la suite, on calculera les puissances tensorielles $S^{n} \bar{E}$.

Pour tout $i \in\{1, \ldots, r\}$, on choisit un élément non-nul $s_{i}$ dans $L_{i}$. Alors $\left(s^{I}\right)_{I \in \mathbb{N}^{r},|I|=n}$ est une base de $S^{n} E_{K}$, où pour tout $I=\left(i_{1}, \ldots, i_{r}\right) \in \mathbb{N}^{r}, s^{I}$ désigne $s_{1}^{i_{1}} \cdots s_{r}^{i_{r}}$. Pour tout $I \in \mathbb{N}^{r},|I|=n$, on désigne par $\bar{L}^{I}$ le sous-espace de $S^{n} E$ engendré par $s^{I}$, muni des métriques induites. Pour toute place finie (resp. infinie) $v,\left(s_{i}\right)_{i=1}^{r}$ est une base $v$-adique (resp. orthogonale) de $E \otimes_{K} \mathbb{C}_{v}$. Par conséquent, $\left(s^{I}\right)_{I \in \mathbb{N}^{r},|I|=n}$ est une base $v$-adique (resp. orthogonale) de $S^{n} E \otimes_{K} \mathbb{C}_{v}$. On obtient donc $S^{n} \bar{E}=\bigoplus_{I \in \mathbb{N}^{r},|I|=n} \bar{L}^{I}$. D'après (18), le degré d'Arakelov de $\bar{L}^{I}, I=\left(i_{1}, \ldots, i_{r}\right)$ se calcule comme la suite

$$
\widehat{\operatorname{deg}}\left(\bar{L}^{I}\right)=-\sum_{v} n_{v} \log \left\|s^{I}\right\|_{v}=\sum_{j=1}^{r}\left(i_{j} \widehat{\operatorname{deg}}\left(\bar{L}_{j}\right)-\frac{d_{K}}{2} \log \left(i_{j} !\right)\right)+\frac{d_{K}}{2} \log (n !)
$$

où on a utilisé les relations $(66)$ et $(67)$ plus haut, et $d_{K}:=[K: \mathbb{Q}]$. On rappelle la formule de Stirling :

$$
\log m !=\log \sqrt{2 \pi}+\frac{1}{2} \log m+m(\log m-1)+\varepsilon_{m},
$$

où $\left.\varepsilon_{m} \in\right] \frac{1}{12 m+1}, \frac{1}{12 m}[$, d'où on obtient

$$
\begin{aligned}
\widehat{\mu}\left(\bar{L}^{I}\right) & =\sum_{j=1}^{r} i_{j}\left(\widehat{\mu}\left(\bar{L}_{j}\right)-\frac{1}{2} \log i_{j}\right)+\frac{1}{2} n \log n+\text { terme d'erreur } \\
& =\sum_{j=1}^{r} i_{j}\left(\widehat{\mu}\left(\bar{L}_{j}\right)-\frac{1}{2} \log \frac{i_{j}}{n}\right)+\text { terme d'erreur, }
\end{aligned}
$$

où la valeur absolue du terme d'erreur est majorée par $\frac{1}{2}(r+1)\left(\log \sqrt{2 \pi}+\frac{1}{2} \log n+1\right)$. Mesure limite. - Soient $\mathscr{X}=\mathbb{P}(E)$ et $\pi: \mathscr{X} \rightarrow$ Spec $\theta_{K}$ le morphisme canonique. On note $\mathscr{L}=\mathscr{\vartheta}_{\mathbb{P}(E)}(1)$. L'espace $\mathscr{X}(\mathbb{C})$ des points complexes de $\mathscr{X}$, muni de la topologie analytique, est une variété analytique complexe. On munit $\mathscr{L}_{\mathbb{C}}$ de la métrique de Fubini-Study, c'est-à-dire la métrique quotient induit par le morphisme universel $\pi_{\mathbb{C}}^{*} E_{\mathbb{C}} \rightarrow \mathscr{L}_{\mathbb{C}}$. On obtient ainsi un fibré inversible hermitien $\overline{\mathscr{L}}$ sur $\mathscr{X}$. En outre, on fixe une mesure de Lebesgue sur $\mathscr{X}_{\sigma}(\mathbb{C})$ qui est proportionnelle à la $(r-1)^{\text {ième }}$ puissance extérieure de la $(1,1)$-forme de Fubini-Study, et qui est de masse totale 1 sur chaque composante connexe de $\mathscr{X}(\mathbb{C})$. Pour tout entier $n \geqslant 1$, soit $\overline{\mathscr{E}}_{n}$ le $\theta_{K}$-module 
$\pi_{*}\left(\mathscr{L}^{\otimes n}\right)$, muni des métriques $L^{2}$. On désigne par $\bar{E}_{n}$ le fibré adélique hermitien correspondant.

Dans la suite, on calculera explicitement les mesures $T_{\frac{1}{n}} \nu_{\bar{E}_{n}}$. La méthode repose sur la forme explicite de $S^{n} \bar{E}$ étudiée plus haut, et un résultat de comparaison entre $\bar{E}_{n}$ et $S^{n} \bar{E}$ établi dans [10]. Observons que $\bar{E}_{n}$ et $S^{n} \bar{E}$ ont le même espace vectoriel sous-jacent, et ont les mêmes métriques en les places finies. Par contre, si on note $\|\cdot\|_{v, 2}$ et $\|\cdot\|_{v}$ la norme hermitienne d'indice $v \in \Sigma_{\infty}$ de $\bar{E}_{n}$ et de $S^{n} \bar{E}$, respectivement, d'après [10, lemme 4.3.6], on a la relation suivante :

$$
\forall x \in E_{n}, x \neq 0, \log \|x\|_{v, 2}=\log \|x\|_{v}-\frac{1}{2} \log \left(\begin{array}{c}
n+r-1 \\
r-1
\end{array}\right) .
$$

Par conséquent, si on désigne par $\bar{L}^{I, 2}$ le sous-fibré inversible adélique de $\bar{E}_{n}$ tel que $L^{I, 2}=L^{I}$, où $I=\left(i_{1}, \ldots, i_{r}\right) \in \mathbb{N}^{r},|I|=n$, alors on a, d'après (69), que

$$
\widehat{\mu}\left(\bar{L}^{I, 2}\right)=\sum_{j=1}^{r} i_{j}\left(\widehat{\mu}\left(\bar{L}_{j}\right)-\frac{1}{2} \log \frac{i_{j}}{n}\right)+O(\log n) .
$$

En outre, la relation $\bar{E}_{n}=\bigoplus_{I \in \mathbb{N}^{r},|I|=n} \bar{L}^{I, 2}$ montre que

$$
T_{\frac{1}{n}} \nu_{\bar{E}_{n}}=\frac{1}{\operatorname{rg} E_{n}} \sum_{I \in \mathbb{N}^{r},|I|=n} \delta_{\frac{1}{n} \widehat{\mu}\left(\bar{L}^{I, 2}\right)} .
$$

On note

$$
\lambda_{I}=\sum_{j=1}^{r} \frac{i_{j}}{n}\left(\widehat{\mu}\left(\bar{L}_{j}\right)-\frac{1}{2} \log \frac{i_{j}}{n}\right), \quad \nu_{n}=\frac{1}{\operatorname{rg} E_{n}} \sum_{I \in \mathbb{N}^{r},|I|=n} \delta_{\lambda_{I}} .
$$

Les suites de mesures $\left(T_{\frac{1}{n}} \nu_{\bar{E}_{n}}\right)$ et $\left(\nu_{n}\right)_{n \geqslant 1}$ convergent vers la même limite que l'on notera $\nu$.

Soit $\Delta_{r}=\left\{\left(x_{1}, \ldots, x_{r}\right) \in \mathbb{R}^{r} \mid x_{1}+\cdots+x_{r}=1\right\}$ le simplexe dans $\mathbb{R}^{r}$ et $\eta$ la mesure de Lebesgue de masse totale 1 sur $\Delta_{r}$. On désigne par $\Phi: \Delta_{r} \rightarrow \mathbb{R}$ l'application

$$
\Phi\left(x_{1}, \ldots, x_{r}\right)=\sum_{j=1}^{r} x_{j} \widehat{\mu}\left(\bar{L}_{j}\right)-\frac{1}{2} x_{j} \log x_{j} .
$$

Initialement la fonction $\Phi$ n'est bien définie que sur $\Delta_{r}$ privé le bord, mais l'égalité $\lim _{x \rightarrow 0} x \log x=0$ nous permet de la prolonger continûment sur $\Delta_{r}$.

Proposition 4.1.10. - Avec les notations ci-dessus, on a $\nu=\Phi_{*} \eta$.

Démonstration. - Par la définition de $\Phi$, pour tout $I=\left(i_{1}, \ldots, i_{r}\right) \in \mathbb{N}^{r}$ tel que $|I|=n$, on a $\lambda_{I}=\Phi\left(i_{1} / n, \ldots, i_{r} / n\right)$. Donc

$$
\frac{1}{\operatorname{rg} E_{n}} \sum_{I \in \mathbb{N}^{r},|I|=n} \delta_{\lambda_{I}}=\frac{1}{\operatorname{rg} E_{n}} \sum_{\underline{b} \in \frac{1}{n} \mathbb{N}^{r} \cap \Delta_{r}} \delta_{\Phi(\underline{b})}=\Phi_{*}\left[\frac{1}{\operatorname{rg} E_{n}} \sum_{\underline{b} \in \frac{1}{n} \mathbb{N}^{r} \cap \Delta_{r}} \delta_{\underline{b}}\right] .
$$

Comme la mesure entre les crochets est la $n^{\text {ième }}$ somme de Riemann sur $\Delta_{r}$, les mesures $\nu_{n}$ convergent vers $\Phi_{*} \eta$ lorsque $n \rightarrow \infty$. 
Considérons maintenant un cas très particulier où $r=2$. Soient $\alpha=\widehat{\operatorname{deg}}\left(\bar{L}_{1}\right)$ et $\beta=\widehat{\operatorname{deg}}\left(\bar{L}_{2}\right)$ respectivement. Dans ce cas-là le simplexe $\Delta_{2}$ est un morceau de droite dans $\mathbb{R}^{2}$ paramétré par $[0,1]$ : un point général de $\Delta_{2}$ est de la forme $\underline{x}(t)=(t, 1-t)$. L'application $\Phi$ envoie $\underline{x}(t)$ vers

$$
f(t):=\alpha t+\beta(1-t)-\frac{1}{2}(t \log t+(1-t) \log (1-t)) .
$$

Comme la mesure de probabilité de Lebesgue sur $\Delta_{2}$ est l'image directe de la distribution uniforme sur [0,1] par l'application de paramétrisation $t \mapsto \underline{x}(t)$, pour toute fonction borélienne $h$ sur $\mathbb{R}$ telle que $h \circ f$ soit intégrable sur $[0,1]$, on a

$$
\int_{\mathbb{R}} h \mathrm{~d} \nu=\int_{0}^{1} h(f(t)) \mathrm{d} t
$$

où $\nu$ est la limite de $\left(T_{\frac{1}{n}} \nu_{E_{n}}\right)_{n \geqslant 1}$. En outre, on a

$$
\lim _{n \rightarrow \infty} \frac{\widehat{\mu}_{\max }\left(\bar{E}_{n}\right)}{n}=\sup _{t \in[0,1]} f(t), \quad \lim _{n \rightarrow \infty} \frac{\widehat{\mu}_{\min }\left(\bar{E}_{n}\right)}{n}=\inf _{t \in[0,1]} f(t) .
$$

Comme $-\frac{1}{2}(t \log t+(1-t) \log (1-t))$ est positif sur $[0,1]$, on obtient $\inf _{t \in[0,1]} f(t)=$ $\min \{\alpha, \beta\}$. Cependant, la fonction $f$ prend sa valeur maximale en

$$
t_{0}=e^{2(\alpha-\beta)} /\left(1+e^{2(\alpha-\beta)}\right),
$$

et

$$
\sup _{t \in[0,1]} f(t)=\frac{1}{2}\left(\log \left(e^{2(\alpha-\beta)}+1\right)+2 \beta\right) .
$$

La comparaison avec le calcul que l'on a mené dans l'exemple 3.1.9 montre que, même si le fibré vectoriel adélique $\bar{E}$ est très simple, les limites des invariants arithmétiques de $\overline{\mathscr{L}}$ peuvent être très compliquées. Par exemple, quand $\alpha=\beta=0$, le fibré vectoriel adélique $\bar{E}$ est semi-stable de pente 0 , mais la mesure limite $\lim _{n \rightarrow \infty} T_{\frac{1}{n}} \nu_{\bar{E}_{n}}$ est l'image directe de la mesure uniforme sur [0,1] par l'application $f(t)=-\frac{1}{2}(t \log t+(1-t) \log (1-t))$, qui n'est pas du tout une mesure de Dirac.

\subsection{Pente maximale asymptotique}

Soit $\pi: \mathscr{X} \rightarrow$ Spec $\theta_{K}$ un morphisme projectif et plat, tel que $\mathscr{X}_{K}$ soit intègre de dimension $d$. Dans le paragraphe précédent, on a établi, pour tout fibré inversible hermitien $X:=\overline{\mathscr{L}}$ sur $\mathscr{X}$ tel que $\mathscr{L}_{K}$ soit effectif, la convergence de $\left(\widehat{\mu}_{\max }\left(\pi_{*} \overline{\mathscr{L}}^{\otimes n}\right) / n\right)_{n \geqslant 1}$, où $\pi_{*} \overline{\mathscr{L}}^{\otimes n}$ désigne le $\theta_{K}$-module $\pi_{*}\left(\mathscr{L}^{\otimes n}\right)$, muni des normes sup. Dans le groupe de Picard $\operatorname{Pic}\left(\mathscr{X}_{K}\right)$, l'intérieur du cône des fibrés inversibles effectifs est le cône des fibrés inversibles gros. On rappelle qu'un fibré inversible $L$ sur $\mathscr{X}_{K}$ est dit gros si son volume, défini comme (cf. [33, théorème 2.2.26])

$$
\operatorname{vol}(L):=\limsup _{n \rightarrow \infty} \frac{\operatorname{rg} H^{0}\left(\mathscr{X}_{K}, L^{\otimes n}\right)}{n^{d} / d !},
$$


est strictement positif. On rappelle aussi que, si un fibré inversible $L$ sur $\mathscr{X}_{K}$ est gros, alors il existe une puissance de $L$ qui peut être décomposée comme le produit tensoriel d'un fibré inversible ample et d'un fibré inversible effectif (cf. [33, 2.2.7]). Pour tout fibré inversible hermitien $\overline{\mathscr{L}}$ sur $\mathscr{X}$ tel que $\mathscr{L}_{K}$ soit gros, on désigne par $\widehat{\mu}_{\max }^{\pi}(\overline{\mathscr{L}})$ la limite de la suite $\left(\widehat{\mu}_{\max }\left(\pi_{*} \overline{\mathscr{L}}^{\otimes n}\right) / n\right)_{n \geqslant 1}$. On montrera que la fonction $\widehat{\mu}_{\max }^{\pi}$ est sur-additive. Cette observation permet d'étendre le domaine de définition de la fonction $\widehat{\mu}_{\text {max }}^{\pi}$ à l'ensemble de tous les fibrés inversibles hermitiens sur $\mathscr{X}$. Enfin, on étudie les propriétés arithmétiques de la fonction $\widehat{\mu}_{\max }^{\pi}(\overline{\mathscr{L}})$.

Notation. - Dans la suite du paragraphe, si $\overline{\mathcal{E}}$ est un fibré vectoriel normé sur $\mathscr{X}$, alors l'expression $\pi_{*}(\overline{\mathscr{E}})$ désigne le $\emptyset_{K}$-module $\pi_{*}(\mathcal{E})$, muni des métriques sup.

4.2.1. Sur-additivité de la pente maximale asymptotique. - Dans ce sousparagraphe, on vérifie que la fonction $\widehat{\mu}_{\max }^{\pi}$ est sur-additive.

LEMME 4.2.1. - Soient $\overline{\mathscr{L}}_{1}$ et $\overline{\mathscr{L}}_{2}$ deux fibrés inversibles hermitiens tels que $\mathscr{L}_{1, K}$ et $\mathscr{L}_{2, K}$ soient effectifs. On note $\overline{\mathscr{E}}_{i}=\pi_{*}\left(\overline{\mathscr{L}}_{i}\right)(i=1,2)$ et $\overline{\mathcal{E}}=\pi_{*}\left(\overline{\mathscr{L}}_{1} \otimes \overline{\mathscr{L}}_{2}\right)$. Alors

$$
\widehat{\mu}_{\max }(\overline{\mathcal{E}}) \geqslant \widehat{\mu}_{\max }\left(\overline{\mathscr{E}}_{1}\right)+\widehat{\mu}_{\max }\left(\overline{\mathscr{E}}_{2}\right)-U\left(\operatorname{rg} \mathcal{E}_{1}, K\right)-U\left(\operatorname{rg} \mathcal{E}_{2}, K\right),
$$

où la constant $U(n, K)$ est définie dans la proposition 4.1.6.

Démonstration. - D'après $(63)$, on a $\widehat{\mu}_{\max }(\overline{\mathcal{E}}) \geqslant-\log \lambda_{1}(\overline{\mathcal{E}})$. Par définition,

$$
-\log \lambda_{1}(\bar{\delta})=-\inf _{0 \neq s \in \mathscr{E}} \max _{\sigma: K \rightarrow \mathbb{C}} \log \|s\|_{\sigma, \text { sup }} \geqslant-\inf _{\substack{0 \neq s_{1} \in \mathscr{E}_{1} \\ 0 \neq s_{2} \in \mathcal{E}_{2}}} \max _{\sigma: K \rightarrow \mathbb{C}} \log \left\|s_{1} \cdot s_{2}\right\|_{\sigma, \text { sup }} .
$$

Pour tout $s_{i} \in \mathcal{E}_{i}(i=1,2)$ et tout $\sigma: K \rightarrow \mathbb{C}$, on a $\left\|s_{1} \cdot s_{2}\right\|_{\sigma, \text { sup }} \leqslant\left\|s_{1}\right\|_{\sigma, \text { sup }} \cdot\left\|s_{2}\right\|_{\sigma, \text { sup }}$, d'où $-\log \lambda_{1}(\overline{\mathcal{E}}) \geqslant-\log \lambda_{1}\left(\bar{\varepsilon}_{1}\right)-\log \lambda_{1}\left(\bar{\varepsilon}_{2}\right)$. Encore d'après (63), on obtient

$$
-\log \lambda_{1}(\overline{\mathscr{E}}) \geqslant \widehat{\mu}_{\max }\left(\overline{\mathscr{E}}_{1}\right)+\widehat{\mu}_{\max }\left(\bar{\varepsilon}_{2}\right)-U\left(\operatorname{rg} \mathcal{E}_{1}, K\right)-U\left(\operatorname{rg} \varepsilon_{2}, K\right),
$$

qui implique (71) puisque $\widehat{\mu}_{\max }(\overline{\mathcal{E}}) \geqslant-\log \lambda_{1}(\overline{\mathcal{E}})$.

Proposition 4.2.2. - Soient $\overline{\mathscr{L}}_{1}$ et $\overline{\mathscr{L}}_{2}$ deux fibrés inversibles hermitiens tels que $\mathscr{L}_{1, K}$ et $\mathscr{L}_{2, K}$ soient gros. Alors

$$
\widehat{\mu}_{\max }^{\pi}\left(\overline{\mathscr{L}}_{1} \otimes \overline{\mathscr{L}}_{2}\right) \geqslant \widehat{\mu}_{\max }^{\pi}\left(\overline{\mathscr{L}}_{1}\right)+\widehat{\mu}_{\max }^{\pi}\left(\overline{\mathscr{L}}_{2}\right) .
$$

Démonstration. - Pour tout entier $n$ suffisamment grand (de sorte que les $\mathscr{L}_{i, K}^{\otimes n}$ soient effectifs), on note $\overline{\mathcal{E}}_{n}^{(i)}=\pi_{*}\left(\overline{\mathscr{L}}_{i}^{\otimes n}\right)(i=1,2)$, et $\overline{\mathcal{E}}_{n}=\pi_{*}\left(\overline{\mathscr{L}}_{1}^{\otimes n} \otimes \overline{\mathscr{L}}_{2}^{\otimes n}\right)$. D'après (71), pour tout entier $n$ assez grand, on a

$$
\widehat{\mu}_{\max }\left(\overline{\mathscr{E}}_{n}\right) \geqslant \widehat{\mu}_{\max }\left(\overline{\mathcal{E}}_{n}^{(1)}\right)+\widehat{\mu}_{\max }\left(\overline{\mathcal{E}}_{n}^{(2)}\right)-U\left(\operatorname{rg} \mathcal{E}_{n}^{(1)}, K\right)-U\left(\operatorname{rg} \mathcal{E}_{n}^{(2)}, K\right)
$$

Si on divise les deux côtés de (73) par $n$ et passe $n$ tendre vers l'infini, d'après la proposition 4.1.6, on obtient (72).

Proposition 4.2.3. - Soient $\overline{\mathscr{L}}$ un fibré inversible hermitien sur $\mathscr{X}$ tel que $\mathscr{L}_{K}$ soit gros, et $\bar{M}$ un fibré inversible hermitien sur $\operatorname{Spec} \theta_{K}$. Alors 
1) Pour tout entier $n \geqslant 1, \widehat{\mu}_{\max }^{\pi}\left(\overline{\mathscr{L}}^{\otimes n}\right)=n \widehat{\mu}_{\max }^{\pi}(\overline{\mathscr{L}})$,

2) $\widehat{\mu}_{\max }^{\pi}\left(\overline{\mathscr{L}} \otimes \pi^{*} \bar{M}\right)=\widehat{\mu}(\bar{M})+\widehat{\mu}_{\max }^{\pi}(\overline{\mathscr{L}})$.

Démonstration. -1$)$ résulte de la définition de $\widehat{\mu}_{\max }^{\pi}(\cdot)$.

2) En effet, on a $\pi_{*}\left(\left(\overline{\mathscr{L}} \otimes \pi^{*}(\bar{M})\right)^{\otimes n}\right)=\pi_{*}\left(\overline{\mathscr{L}}^{\otimes n}\right) \otimes \bar{M}^{\otimes n}$. D'après (37), on a

$$
\widehat{\mu}_{\max }\left(\pi_{*}\left(\overline{\mathscr{L}} \otimes \pi^{*}(\bar{M})\right)^{\otimes n}\right)=\widehat{\mu}_{\max }\left(\pi_{*}\left(\overline{\mathscr{L}}^{\otimes n}\right)\right)+n \widehat{\mu}(\bar{M}) .
$$

Par passage à la limite on obtient $\widehat{\mu}_{\max }^{\pi}\left(\overline{\mathscr{L}} \otimes \pi^{*} \bar{M}\right)=\widehat{\mu}(\bar{M})+\widehat{\mu}_{\max }^{\pi}(\overline{\mathscr{L}})$.

4.2.2. Pente maximale asymptotique d'un fibré inversible hermitien quelconque. - La sur-additivité de $\widehat{\mu}_{\max }^{\pi}$ obtenue dans le sous-paragraphe précédent permet d'étendre le domaine de définition de la fonction $\widehat{\mu}_{\max }^{\pi}(\overline{\mathscr{L}})$ à l'espace de tous les fibrés inversibles hermitiens sur $\mathscr{X}$. Dans le reste de la section, le symbole $\Theta$ désigne l'espace des fibrés inversibles hermitiens $\overline{\mathscr{L}}$ sur $\mathscr{X}$ tels que $\mathscr{L}_{K}$ soit gros.

DÉfinition 4.2.4. - Soient $\overline{\mathscr{L}}$ et $\overline{\mathscr{L}}$ deux fibrés inversibles hermitiens sur $\mathscr{X}$. On suppose que $\overline{\mathscr{L}} \in \Theta$. Il existe alors un entier $n_{0}(\mathscr{L}, \mathscr{L})>0$ tel que $\mathscr{L}_{K} \otimes \mathscr{L}_{K}^{\otimes n}$ soit gros quel que soit $n \geqslant n_{0}(\mathscr{L}, \mathscr{L})$ (cf. [33, 2.2.24]). On définit, pour tout entier $n \geqslant n_{0}(\mathscr{L}, \mathscr{L})$,

$$
A_{n}(\overline{\mathscr{L}}, \overline{\mathscr{L}})=\widehat{\mu}_{\max }^{\pi}\left(\overline{\mathscr{L}} \otimes \overline{\mathscr{L}}^{\otimes n}\right)-n \widehat{\mu}_{\max }^{\pi}(\overline{\mathscr{L}})
$$

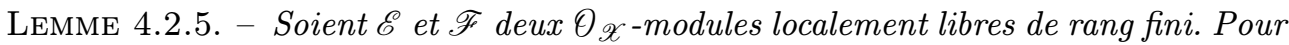
tout homomorphisme $f: \mathscr{E}_{K} \rightarrow \mathscr{F}_{K}$, il existe un élément non-nul a $\in \theta_{K}$ tel que af se relève en un homomorphisme de $\mathscr{E}$ vers $\mathscr{F}$. Si de plus $f$ est injectif, l'homomorphisme relevé peut être choisi injectif.

Démonstration. - Soit $\eta: \operatorname{Spec} K \rightarrow \operatorname{Spec} \theta_{K}$ le point générique. C'est un morphisme plat. Soit $X:=\mathscr{X}_{K}$. On désigne par $p: X \rightarrow \operatorname{Spec} K$ et $q: X \rightarrow \mathscr{X}$ les morphismes canoniques, qui s'insèrent dans un carré cartésien:

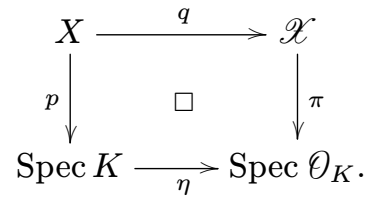

D'après [26] III, 1.4.15, pour tout $\Theta_{\mathscr{X}}$-module quasi-cohérent $\mathscr{G}$, l'homomorphisme canonique $\eta^{*} \pi_{*} \mathscr{G} \rightarrow p_{*} q^{*} \mathscr{G}$ est un isomorphisme. Par conséquent, on a un isomorphisme canonique $H^{0}\left(X, \mathscr{G}_{K}\right) \cong H^{0}(\mathscr{X}, \mathscr{G})_{K}$. Si $\mathscr{E}$ et $\mathscr{F}$ sont deux $\bigoplus_{\mathscr{X}}$ modules localement libres de rang fini, alors $\operatorname{Hom}_{\vartheta_{\mathscr{X}}}(\mathscr{E}, \mathscr{F})$ s'identifie à l'espace des sections de $\mathscr{E}^{\vee} \otimes \mathscr{F}$ au-dessus de $\mathscr{X}$. D'autre part,

$$
\operatorname{Hom}_{\vartheta_{X}}\left(\mathscr{E}_{K}, \mathscr{F}_{K}\right) \cong H^{0}\left(X, \mathscr{E}_{K}^{\vee} \otimes \mathscr{F}_{K}\right)=H^{0}\left(\mathscr{X}, \mathscr{E}^{\vee} \otimes \mathscr{F}_{K}\right.
$$


Par conséquent, pour tout homomorphisme $f: \mathscr{E}_{K} \rightarrow \mathscr{F}_{K}$, il existe un élément nonnul $a \in \theta_{K}$ tel que af se relève en un homomorphisme de $\mathscr{E} \rightarrow \mathscr{F}$, autrement dit, l'image de l'homomorphisme composé

$$
\mathscr{E} \stackrel{a}{\longrightarrow} \mathscr{E} \longrightarrow q_{*} \mathscr{E}_{K} \stackrel{q_{*} f}{\longrightarrow} q_{*} \mathscr{F}_{K}
$$

est dans $\mathscr{F}$. Cet homomorphisme est injectif lorsque $f$ est injectif.

Soit $\overline{\mathscr{E}}$ un fibré vectoriel normé sur $\mathscr{X}$. On dit qu'une section $s \in H^{0}(\mathscr{X}, \mathcal{E})$ est effective si $\max _{\sigma: K \rightarrow \mathbb{C}}\|s\|_{\sigma, \text { sup }} \leqslant 1$. On dit que $s$ est strictement effective si l'inégalité est stricte, c'est-à-dire $\max _{\sigma: K \rightarrow \mathbb{C}}\|s\|_{\sigma, \text { sup }}<1$. Toute section $s \in H^{0}(\mathscr{X}, \mathcal{E})$ correspond à un homomorphisme $\varphi: \theta_{\mathscr{X}} \rightarrow \mathcal{E}$. Si on munit $\Theta_{\mathscr{X}}$ des métriques hermitiennes habituelles, alors $s$ est effective si et seulement si $\max _{\sigma: K \rightarrow \mathbb{C}} \sup _{x \in X_{\sigma}(\mathbb{C})}\left\|\varphi_{x}\right\|_{\sigma} \leqslant 1$; elle est strictement effective si et seulement si $\max _{\sigma: K \rightarrow \mathbb{C}} \sup _{x \in X_{\sigma}(\mathbb{C})}\left\|\varphi_{x}\right\|_{\sigma}<1$.

Soient $\overline{\mathscr{E}}_{1}$ et $\overline{\mathscr{E}}_{2}$ deux fibrés vectoriels normés sur $\mathscr{X}$, et $f: \mathcal{E}_{1, K} \rightarrow \mathcal{E}_{2, K}$ un homomorphisme. On a démontré dans le lemme 4.2.5 qu'il existe $a \in \theta_{K}$ tel que $a f$ se relève en un homomorphisme de $\mathcal{E}_{1}$ vers $\mathcal{E}_{2}$. Par conséquent, pour tout sauf un nombre fini de place finie $\mathfrak{p}$, l'homomorphisme $f_{\mathbb{C}_{\mathfrak{p}}}$ se relève en un homomorphisme de $\mathcal{E}_{1, \widehat{\vartheta}_{\mathfrak{p}}}$ vers $\mathcal{E}_{2, \widehat{\vartheta}_{\mathfrak{p}}}$. Pour tout $\mathfrak{p} \in \Sigma_{f}$, on désigne par $h_{\mathfrak{p}}(f)$ la borne inférieure des valeurs - $\log |b|_{\mathfrak{p}}$, où $b$ parcourt l'ensemble des éléments dans $\mathbb{C}_{\mathfrak{p}}$ tels que $b f_{\mathbb{C}_{p}}$ se relève en un homomorphisme de $\mathcal{E}_{1, \widehat{\theta}_{\mathfrak{p}}}$ vers $\mathcal{E}_{2, \widehat{\vartheta}_{\mathfrak{p}}}$. L'argument ci-dessus montre que $h_{\mathfrak{p}}<+\infty$ et que $h_{\mathfrak{p}}(f) \leqslant 0$ pour toute sauf un nombre fini de places finies $\mathfrak{p}$. Si $v$ est une place infinie de $K$ qui correspond à un plongement $\sigma: K \rightarrow \mathbb{C}$, on désigne par $h_{v}(f)$ ou $h_{\sigma}(f)$ le nombre réel $\sup _{\mathscr{C}} \log \left\|f_{x}\right\|_{\sigma}$. Le nombre $h_{v}(f)\left(v \in \Sigma_{f} \cup \Sigma_{\infty}\right)$ est appelé la hauteur locale de $f$ en $v$. On définit la hauteur de $f$ comme le nombre

$$
h(f)=\frac{1}{[K: \mathbb{Q}]} \sum_{v \in \Sigma} n_{v} h_{v}(f) \in[-\infty,+\infty[.
$$

On observe que, lorsque $\mathscr{X}=\operatorname{Spec} \theta_{K}$, la définition de $h_{v}(f)$ et de $h(f)$ coïncide avec celle à la fin de $§ 2.1 .1$.

LEMme 4.2.6. - Soient $\overline{\mathcal{E}}_{1}$ et $\overline{\mathcal{E}}_{2}$ deux fibrés vectoriels normés sur $\mathscr{X}$, et $f: \mathcal{E}_{1, K} \rightarrow$ $\mathcal{E}_{2, K}$ un homomorphisme. Soit $\varphi: H^{0}\left(\mathscr{X}_{K}, \mathcal{E}_{1, K}\right) \rightarrow H^{0}\left(\mathscr{X}_{K}, \mathcal{E}_{2, K}\right)$ l'application $K$ linéaire induite par $f$. Alors on a $h_{v}(\varphi) \leqslant h_{v}(f)$ quel que soit $v \in \Sigma_{f} \cup \Sigma_{\infty}$, où on a considéré $H^{0}\left(\mathscr{X}_{K}, \mathscr{L}_{i, K}\right)$ comme la fibre générique de $\pi_{*}\left(\overline{\mathscr{E}}_{i}\right)(i=1,2)$. Si de plus $\varphi$ est injectif, alors

$$
\widehat{\mu}_{\max }\left(\pi_{*}\left(\bar{\varepsilon}_{1}\right)\right) \leqslant \widehat{\mu}_{\max }\left(\pi_{*}\left(\bar{\varepsilon}_{2}\right)\right)+h(f) .
$$

Démonstration. - Soient $\mathfrak{p} \in \Sigma_{f}$ et

$$
\varphi_{\mathfrak{p}}: H^{0}\left(\mathscr{X}_{\mathbb{C}_{\mathfrak{p}}}, \mathcal{E}_{1, \mathbb{C}_{\mathfrak{p}}}\right) \rightarrow H^{0}\left(\mathscr{X}_{\mathbb{C}_{\mathfrak{p}}}, \mathcal{E}_{2, \mathbb{C}_{\mathfrak{p}}}\right)
$$


l'homomorphisme induit par $\varphi$. Par définition, $\pi_{*}\left(\mathcal{E}_{1}\right) \otimes \widehat{\mathscr{\theta}}_{\mathfrak{p}}\left(\right.$ resp. $\left.\pi_{*}\left(\mathcal{E}_{2}\right) \otimes \widehat{\mathscr{\theta}}_{\mathfrak{p}}\right)$ est le disque fermé de rayon 1 dans $H^{0}\left(\mathscr{X}_{\mathbb{C}_{\mathfrak{p}}}, \mathcal{E}_{1, \mathbb{C}_{\mathfrak{p}}}\right)\left(\operatorname{resp} . H^{0}\left(\mathscr{X}_{\mathbb{C}_{\mathfrak{p}}}, \mathcal{E}_{2, \mathbb{C}_{\mathfrak{p}}}\right)\right)$. Si $b \in \mathbb{C}_{\mathfrak{p}}$ est tel que $b f_{\mathbb{C}_{\mathfrak{p}}}$ se relève en un homomorphisme de $\mathcal{E}_{1, \widehat{\mathscr{O}}_{\mathfrak{p}}}$ vers $\mathcal{E}_{2, \widehat{\vartheta}_{\mathfrak{p}}}$, alors $b \varphi_{\mathfrak{p}}$ se relève en un homomorphisme de $\pi_{*}\left(\mathcal{E}_{1}\right) \otimes \widehat{\theta}_{\mathfrak{p}}$ vers $\pi_{*}\left(\mathcal{E}_{2}\right) \otimes \widehat{\theta}_{\mathfrak{p}}$. Donc $\left\|b \varphi_{\mathfrak{p}}\right\|_{\mathfrak{p}} \leqslant 1$, d'où $\left\|\varphi_{\mathfrak{p}}\right\|_{\mathfrak{p}} \leqslant|b|_{\mathfrak{p}}^{-1}$. On obtient donc $\log \left\|\varphi_{\mathfrak{p}}\right\|_{\mathfrak{p}} \leqslant h_{\mathfrak{p}}(f)$. Supposons maintenant que $\sigma: K \rightarrow \mathbb{C}$ est un plongement. Pour tout élément $s \in H^{0}\left(\mathscr{X}_{\sigma}(\mathbb{C}), \mathcal{E}_{1, \sigma}\right)$, on a

$$
\left\|\varphi_{\sigma}(s)\right\|_{\sigma, \sup }=\sup _{x \in \mathscr{X}_{\sigma}(\mathbb{C})}\left\|f(s)_{x}\right\| \leqslant e^{h_{\sigma}(f)}\|s\|_{\sigma, \mathrm{sup}}
$$

qui implique que $\log \left\|\varphi_{\sigma}\right\|_{\sigma} \leqslant h_{\sigma}(f)$. Par conséquent, on a $h(\varphi) \leqslant h(f)$.

Si $f$ est injectif, l'homomorphisme $\varphi$ est injectif. L'inégalité (75) résulte donc de l'inégalité de pentes (22).

Remarque 4.2.7. - On garde les notations du lemme 4.2.6.

(1) Si l'homomorphisme $f$ lui-même se relève en un homomorphisme de $\mathcal{E}_{1}$ vers $\mathcal{E}_{2}$, alors $h_{\mathfrak{p}}(f) \leqslant 0$ quel que soit $\mathfrak{p} \in \Sigma_{f}$. Donc on obtient

$$
h(f) \leqslant \frac{1}{[K: \mathbb{Q}]} \sum_{\sigma: K \rightarrow \mathbb{C}} h_{\sigma}(f) .
$$

(2) Si $\overline{\mathscr{L}}$ est un autre fibré inversible hermitien, et si $g: \mathcal{E}_{1, K} \otimes \mathscr{L}_{K} \rightarrow \mathcal{E}_{2, K} \otimes \mathscr{L}_{K}$ est l'homomorphisme induit par $f$, alors on a $h_{v}(g)=h_{v}(f)$ pour toute $v \in \Sigma_{f} \cup \Sigma_{\infty}$.

(3) Si $\overline{\mathscr{L}_{1}^{\prime}}$ et $\overline{\mathscr{L}_{2}^{\prime}}$ sont deux fibrés inversibles hermitiens et si $f^{\prime}: \mathscr{L}_{1, K}^{\prime} \rightarrow \mathscr{L}_{2, K}^{\prime}$ est un homomorphisme, alors $h_{v}\left(f \otimes f^{\prime}\right) \leqslant h_{v}(f)+h_{v}\left(f^{\prime}\right)$ pour toute $v \in \Sigma_{f} \cup \Sigma_{\infty}$. En particulier, si $\mathcal{E}_{1}$ et $\mathcal{E}_{2}$ sont de rang 1 , alors pour tout entier $n \geqslant 1$ et toute $v \in \Sigma_{f} \cup \Sigma_{\infty}$, on a $h_{v}\left(f^{\otimes n}\right) \leqslant n h_{v}(f)$. Dans ce cas-là, si on applique le lemme 4.2.6 à $f^{\otimes n}: \mathcal{E}_{1, K}^{\otimes n} \rightarrow \mathcal{E}_{2, K}^{\otimes n}$, on obtient que, la hauteur de l'homomorphisme $\varphi_{n}: H^{0}\left(\mathscr{X}_{K}, \mathcal{E}_{1, K}^{\otimes n}\right) \rightarrow H^{0}\left(\mathscr{X}_{K}, \mathcal{E}_{2, K}^{\otimes n}\right)$ induit par $f^{\otimes n}$ est majorée par $n h(f)$.

(4) Soit $\phi$ une fonction continue sur $\mathscr{X}(\mathbb{C})$ qui est invariante par la conjugaison complexe. Soit $\overline{\vartheta_{\mathscr{X}}(\phi)}$ le fibré inversible hermitien sur $\mathscr{X}$ dont le fibré inversible sous-jacent est le fibré trivial $\Theta_{\mathscr{X}}$ et tel que la norme de la section unitaire $\mathbf{1}$ en $x \in \mathscr{X}(\mathbb{C})$ est $e^{-\phi(x)}$. Alors la hauteur locale en $\sigma: K \rightarrow \mathbb{C}$ de l'homomorphisme d'identité $\overline{\theta_{\mathscr{X}}} \rightarrow \overline{\theta_{\mathscr{X}}(\phi)}$ est égale à la valeur maximale de la restriction de $-\phi$ à $\mathscr{X}_{\sigma}(\mathbb{C})$. En particulier, si $\phi=a$ est une fonction constante, alors la hauteur de l'homomorphisme d'identité $\overline{\theta_{\mathscr{X}}} \rightarrow \overline{\vartheta_{\mathscr{X}}(a)}$ est $-a$.

(5) Si $\overline{\mathscr{E}}_{i}(i=1,2,3)$ sont des fibrés inversibles hermitiens sur $\mathscr{X}, f: \mathcal{E}_{1, K} \rightarrow \mathcal{E}_{2, K}$ et $g: \mathcal{E}_{2, K} \rightarrow \mathcal{E}_{3, K}$ sont des homomorphismes, alors $h_{v}(g f) \leqslant h_{v}(g)+h_{v}(f)$ pour toute $v \in \Sigma_{f} \cup \Sigma_{\infty}$.

(6) Soient $\overline{\mathscr{E}}$ un fibré vectoriel normé sur $\mathscr{X}$ et $g: \theta_{\mathscr{X}} \rightarrow \mathcal{E}$ un homomorphisme, alors $g$ correspond à une section effective (resp. strictement effective) de $\bar{\varepsilon}$ si et seulement si $h_{\sigma}(g) \leqslant 0$ (resp. $h_{\sigma}(g)<0$ ) pour tout $\sigma: K \rightarrow \mathbb{C}$.

(7) Pour tout fibré vectoriel normé $\overline{\mathcal{E}}$ sur $\mathscr{X}$, on a

$\log \lambda_{1}\left(\pi_{*}(\mathcal{E})\right)=\inf \left\{a \in \mathbb{R} \mid \overline{\mathcal{E}} \otimes \overline{\Theta_{\mathscr{X}}(a)}\right.$ admet une section effective non-nulle $\}$. 
Si l'homomorphisme $f$ est injectif, et se relève en un homomorphisme de $\mathcal{E}_{1}$ vers $\mathscr{E}_{2}$, et si $h_{\sigma}(f) \leqslant a$ quel que soit $\sigma: K \rightarrow \mathbb{C}$, alors $\lambda_{1}\left(\pi_{*}\left(\overline{\mathscr{E}}_{1}\right)\right) \geqslant e^{-a} \lambda_{1}\left(\pi_{*}\left(\overline{\mathscr{E}}_{2}\right)\right)$.

Proposition 4.2.8. - Soit $\overline{\mathscr{L}}$ un fibré inversible hermitien sur $\mathscr{X}$.

1) pour tout $\overline{\mathscr{L}} \in \Theta$, la suite $\left(A_{n}(\overline{\mathscr{L}}, \overline{\mathscr{L}})\right)_{n \geqslant n_{0}(\mathscr{L}, \mathscr{L})}$ est croissante et converge vers une limite dans $\mathbb{R}$;

2) si $\bar{M}$ est un fibré inversible hermitien sur $\operatorname{Spec} \theta_{K}, \overline{\mathscr{L}} \in \Theta$, alors

$$
A_{n}\left(\overline{\mathscr{L}}, \overline{\mathscr{L}} \otimes \pi^{*} \bar{M}\right)=A_{n}(\overline{\mathscr{L}}, \overline{\mathscr{L}})
$$

quel que soit $n \geqslant n_{0}(\mathscr{L}, \mathscr{L})$;

3) si $\mathscr{L}_{K}$ est gros, alors

$$
\widehat{\mu}_{\max }^{\pi}(\overline{\mathscr{L}})=\inf _{\mathscr{L} \in \Theta} \lim _{n \rightarrow+\infty} A_{n}(\overline{\mathscr{L}}, \overline{\mathscr{L}})
$$

4) pour tout fibré inversible hermitien $\bar{M}$ sur $\operatorname{Spec} \theta_{K}$, on a

$$
\widehat{\mu}(\bar{M})=\inf _{\overline{\mathscr{L}} \in \Theta} \lim _{n \rightarrow+\infty} A_{n}\left(\pi^{*}(\bar{M}), \overline{\mathscr{L}}\right) .
$$

Démonstration. - 1) D'après la proposition 4.2.2, pour tout entier $n \geqslant n_{0}(\mathscr{L}, \mathscr{L})$,

$$
\widehat{\mu}_{\max }^{\pi}\left(\overline{\mathscr{L}} \otimes \overline{\mathscr{L}}^{\otimes(n+1)}\right) \geqslant \widehat{\mu}_{\max }^{\pi}\left(\overline{\mathscr{L}} \otimes \overline{\mathscr{L}}^{\otimes n}\right)+\widehat{\mu}_{\max }^{\pi}(\overline{\mathscr{L}}) .
$$

Donc $A_{n+1}(\overline{\mathscr{L}}, \overline{\mathscr{L}}) \geqslant A_{n}(\overline{\mathscr{L}}, \overline{\mathscr{L}})$.

Comme $\mathscr{L}_{K}$ est gros, une de ses puissances tensorielles s'écrit comme le produit tensoriel d'un fibré inversible ample et d'un fibré inversible effectif. Donc il existe un entier $m \geqslant 1$ et un homomorphisme injectif $\varphi$ de $\mathscr{L}_{K}$ vers $\mathscr{L}_{K}^{\otimes m}$ (cf. proposition $1.4 .31))$. Pour tout entier $n \geqslant n_{0}(\mathscr{L}, \mathscr{L})$, soit $\varphi_{n}: \mathscr{L}_{K} \otimes \mathscr{L}_{K}^{\otimes n} \rightarrow \mathscr{L}_{K}^{\otimes(m+n)}$ l'homomorphisme induit par $\varphi$, qui est également injectif. D'après l'inégalité (75), pour tout entier $u \geqslant 1$, on a

$$
\widehat{\mu}_{\max }\left(\pi_{*}\left(\overline{\mathscr{L}}^{\otimes u} \otimes \overline{\mathscr{L}}^{\otimes u n}\right)\right) \leqslant \widehat{\mu}_{\max }\left(\pi_{*}\left(\overline{\mathscr{L}}^{\otimes u(n+m)}\right)\right)+h\left(\varphi_{n}^{\otimes u}\right) .
$$

D'après la remarque $4.2 .7(3)$, on a $h\left(\varphi_{n}^{\otimes u}\right) \leqslant u h\left(\varphi_{n}\right) \leqslant u h(\varphi)$. Par passage à la limite, on obtient

$$
\widehat{\mu}_{\max }^{\pi}\left(\overline{\mathscr{L}} \otimes \overline{\mathscr{L}}^{\otimes n}\right) \leqslant \widehat{\mu}_{\max }^{\pi}\left(\overline{\mathscr{L}}^{\otimes(m+n)}\right)+h(\varphi) .
$$

D'après la proposition 4.2.31), on a

$$
A_{n}(\overline{\mathscr{L}}, \overline{\mathscr{L}}) \leqslant m \widehat{\mu}_{\max }^{\pi}(\overline{\mathscr{L}})+h(\varphi) .
$$

Par conséquent, la suite $\left(A_{n}(\overline{\mathscr{L}}, \overline{\mathscr{L}})\right)_{n \geqslant n_{0}(\mathscr{L}, \mathscr{L})}$ est bornée supérieurement, donc converge dans $\mathbb{R}$.

2) En effet, pour tout $n \geqslant n_{0}(\mathscr{L}, \mathscr{L})$, on a, d'après la proposition 4.2.32), $A_{n}\left(\overline{\mathscr{L}}, \overline{\mathscr{L}} \otimes \pi^{*}(\bar{M})\right)=\widehat{\mu}_{\max }^{\pi}\left(\overline{\mathscr{L}} \otimes \overline{\mathscr{L}}^{\otimes n} \otimes \pi^{*}\left(\bar{M}^{\otimes n}\right)\right)-n \widehat{\mu}_{\max }^{\pi}\left(\overline{\mathscr{L}} \otimes \pi^{*}(\bar{M})\right)=A_{n}(\overline{\mathscr{L}}, \overline{\mathscr{L}})$.

3) D'après la proposition 4.2 .31$)$, on a $A_{n}(\overline{\mathscr{L}}, \overline{\mathscr{L}})=\widehat{\mu}_{\max }^{\pi}(\overline{\mathscr{L}})$, donc

$$
\widehat{\mu}_{\max }^{\pi}(\overline{\mathscr{L}}) \geqslant \inf _{\overline{\mathscr{L}} \in \Theta} \lim _{n \rightarrow+\infty} A_{n}(\overline{\mathscr{L}}, \overline{\mathscr{L}}) .
$$


D'autre part, pour tout $\overline{\mathscr{L}} \in \Theta$, d'après la proposition 4.2 .2 , on a l'estimation

$$
A_{n}(\overline{\mathscr{L}}, \overline{\mathscr{L}}) \geqslant \widehat{\mu}_{\max }^{\pi}(\overline{\mathscr{L}})+\widehat{\mu}_{\max }^{\pi}\left(\overline{\mathscr{L}}^{\otimes n}\right)-n \widehat{\mu}_{\max }^{\pi}(\overline{\mathscr{L}})=\widehat{\mu}_{\max }^{\pi}(\overline{\mathscr{L}}) .
$$

Par conséquent, $\lim _{n \rightarrow+\infty} A_{n}(\overline{\mathscr{L}}, \overline{\mathscr{L}}) \geqslant \widehat{\mu}_{\max }^{\pi}(\overline{\mathscr{L}})$.

4) En effet, pour tout $\overline{\mathscr{L}} \in \Theta$, on a, d'après la proposition 4.2.32),

$$
A_{n}\left(\pi^{*}(\bar{M}), \overline{\mathscr{L}}\right)=\widehat{\mu}_{\max }^{\pi}\left(\pi^{*}(\bar{M}) \otimes \overline{\mathscr{L}}^{\otimes n}\right)-n \widehat{\mu}_{\max }^{\pi}(\overline{\mathscr{L}})=\widehat{\mu}(\bar{M}) .
$$

DÉfinition 4.2.9. - Soit $\overline{\mathcal{L}}$ un fibré inversible hermitien sur $\mathscr{X}$. On appelle pente maximale asymptotique de $\overline{\mathcal{L}}$ relativement à $\pi$ la valeur

$$
\widehat{\mu}_{\max }^{\pi}(\overline{\mathscr{L}}):=\inf _{\overline{\mathscr{L}} \in \Theta} \lim _{n \rightarrow+\infty} A_{n}(\overline{\mathscr{L}}, \overline{\mathscr{L}}) \in[-\infty,+\infty[.
$$

La proposition 4.2.8 3) montre que cette fonction généralise la fonction de la pente maximale asymptotique initialement définie sur $\Theta$.

La proposition suivante montre que la fonction $\widehat{\mu}_{\max }^{\pi}$ prolongée préserve les propriétés de la fonction initiale.

Proposition 4.2.10. - Soient $\overline{\mathcal{L}}, \overline{\mathscr{L}}_{1}$ et $\overline{\mathscr{L}}_{2}$ trois fibrés inversibles hermitiens sur $\mathscr{X}$, et $\bar{M}$ un fibré inversible hermitien sur $\operatorname{Spec} \theta_{K}$. On a

1) $\widehat{\mu}_{\max }^{\pi}\left(\overline{\mathscr{L}}_{1} \otimes \overline{\mathscr{L}}_{2}\right) \geqslant \widehat{\mu}_{\max }^{\pi}\left(\overline{\mathscr{L}}_{1}\right)+\widehat{\mu}_{\max }^{\pi}\left(\overline{\mathscr{L}}_{2}\right)$;

2) $\widehat{\mu}_{\max }^{\pi}\left(\overline{\mathscr{L}}^{\otimes n}\right)=n \widehat{\mu}_{\max }^{\pi}(\overline{\mathscr{L}})$ pour tout entier $n \geqslant 1$;

3) $\widehat{\mu}_{\max }^{\pi}\left(\overline{\mathscr{L}} \otimes \pi^{*}(\bar{M})\right)=\widehat{\mu}_{\max }^{\pi}(\overline{\mathscr{L}})+\widehat{\mu}(\bar{M})$;

4) si $f: \mathscr{L}_{1, K} \rightarrow \mathscr{L}_{2, K}$ est un homomorphisme non-nul, alors

$$
\widehat{\mu}_{\max }^{\pi}\left(\overline{\mathscr{L}}_{1}\right) \leqslant \widehat{\mu}_{\max }^{\pi}\left(\overline{\mathscr{L}}_{2}\right)+h(f) .
$$

Démonstration. - Pour tout fibré inversible hermitien $\overline{\mathscr{L}}$ sur $\mathscr{X}$ tel que $\mathscr{L}_{K}$ soit gros, et tout entier $m$ suffisamment grand, on a, d'après les propositions 4.2 .2 et 4.2 .8 et le lemme 4.2.6, que

$$
\begin{gathered}
A_{2 m}\left(\overline{\mathscr{L}}_{1} \otimes \overline{\mathscr{L}}_{2}, \overline{\mathscr{L}}\right) \geqslant A_{m}\left(\overline{\mathscr{L}}_{1}, \overline{\mathscr{L}}\right)+A_{m}\left(\overline{\mathscr{L}}_{2}, \overline{\mathscr{L}}\right), \\
A_{m n}\left(\overline{\mathscr{L}}^{\otimes n}, \overline{\mathscr{L}}\right)=n A_{m}(\overline{\mathscr{L}}, \overline{\mathscr{L}}), \\
A_{m}\left(\overline{\mathscr{L}} \otimes \pi^{*}(\bar{M}), \overline{\mathscr{L}}\right)=A_{m}(\overline{\mathscr{L}}, \overline{\mathscr{L}})+\widehat{\mu}(\bar{M}), \\
A_{m}\left(\overline{\mathscr{L}}_{1}, \overline{\mathscr{L}}\right) \leqslant A_{m}\left(\overline{\mathscr{L}}_{2}, \overline{\mathscr{L}}\right)+h(f) .
\end{gathered}
$$

Par passage à la limite, on obtient les (in)égalités annoncées. 
4.2.3. Pente maximale asymptotique d'un fibré vectoriel hermitien. - La notion de pente maximale asymptotique peut s'étendre naturellement pour tous les fibrés vectoriels hermitien sur $\mathscr{X}$ par passage au faisceau canonique du fibré projectif, muni des métriques de Fubini-Study.

DÉFInition 4.2.11. - Soit $\bar{E}$ un fibré vectoriel hermitien non-nul sur $\mathscr{X}$. On appelle pente maximale asymptotique arithmétique relativement à $\pi$ de $\bar{E}$ la valeur

$$
\widehat{\mu}_{\max }^{\pi}(\bar{E}):=\widehat{\mu}_{\max }^{\pi p}\left(\overline{\theta_{E}(1)}\right),
$$

où $p: \mathbb{P}(E) \rightarrow \mathscr{X}$ est le morphisme canonique et où les métriques sur $\bigoplus_{E}(1)$ sont des métriques de Fubini-Study.

Remarque 4.2.12. - Dans le cas où $\mathscr{X}=\operatorname{Spec} \theta_{K}$ et où $\pi$ est le morphisme d'identité, la pente maximale asymptotique $\widehat{\mu}_{\max }^{\pi}(\bar{E})$ est toujours plus grand que $\widehat{\mu}_{\max }(\bar{E})$ (cf. [22, remarque 7.5]). Voir loc. cit. théorème 7.1 pour une estimation de $\widehat{\mu}_{\max }\left(S^{n} \bar{E}\right)$.

Proposition 4.2.13. - Avec les notations de la définition 4.2.11, si $E_{K}$ est gros (c'est-à-dire que $\theta_{E}(1)_{K}$ est gros), alors

$$
\widehat{\mu}_{\max }^{\pi}(\bar{E})=\lim _{n \rightarrow+\infty} \frac{1}{n} \widehat{\mu}_{\max }\left(\pi_{*}\left(S^{n} \bar{E}\right)\right) .
$$

Démonstration. - Soit $r$ le rang de E. Par définition

$$
\widehat{\mu}_{\max }^{\pi}(\bar{E})=\lim _{n \rightarrow+\infty} \frac{1}{n} \widehat{\mu}_{\max }\left((\pi p)_{*}\left(\overline{\theta_{E}(1)}\right)\right) .
$$

Pour tout entier $n \geqslant 1$ et tout $\sigma: K \rightarrow \mathbb{C}$, on désigne par $\|\cdot\|_{\sigma, L^{2}}$ la métrique $L^{2}$ $\operatorname{sur} S^{n} E_{\sigma, \mathbb{C}}$ par rapport à la métrique de Fubini-Study sur $\Theta_{E}(1)_{\sigma, \mathbb{C}}$, et par $\|\cdot\|_{\sigma}$ la métrique produit symétrique sur $S^{n} E_{\sigma, \mathbb{C}}$. D'après [10] Lemma 4.3.6, on a la relation

$$
\|s\|_{\sigma}^{2}=\left(\begin{array}{c}
n+r-1 \\
n
\end{array}\right)\|s\|_{\sigma, L^{2}}^{2}
$$

pour tout $x \in X_{\sigma}(\mathbb{C})$ et tout $s \in x^{*}\left(S^{n} E\right)$. Par conséquent, on a

$$
\widehat{\mu}_{\max }\left(\pi_{*}\left(S^{n} E,\left(\|\cdot\|_{\sigma}\right)\right)\right)=\widehat{\mu}_{\max }\left(\pi_{*}\left(S^{n} E,\left(\|\cdot\|_{\sigma, L^{2}}\right)\right)\right)-\frac{1}{2[K: \mathbb{Q}]} \log \left(\begin{array}{c}
n+r-1 \\
n
\end{array}\right) .
$$

Par passage à la limite on obtient (cf. la remarque 4.1.9, voir aussi le lemme 4.2.26)

$$
\widehat{\mu}_{\max }^{\pi}(\bar{E})=\lim _{n \rightarrow+\infty} \frac{1}{n} \widehat{\mu}_{\max }\left(\pi_{*}\left(S^{n} \bar{E}\right)\right) .
$$


4.2.4. Lien avec une condition d'annulation. - La négativité de la pente maximale asymptotique d'un fibré inversible hermitien est liée à l'absence de section globale effective de certains fibrés vectoriels normés.

Proposition 4.2.14. - Soit $\overline{\mathscr{L}}$ un fibré inversible hermitien sur $\mathscr{X}$.

1) Si $\widehat{\mu}_{\max }^{\pi}(\overline{\mathscr{L}})>0$, alors $\widehat{\mu}_{\max }^{\pi}\left(\overline{\mathscr{L}}^{\vee}\right)<0$.

2) Si $\widehat{\mu}_{\max }^{\pi}(\overline{\mathscr{L}})<0$, alors $\mathscr{L}$ n'a pas de section effective non-nulle;

3) Si $\widehat{\mu}_{\max }^{\pi}(\overline{\mathscr{L}}) \leqslant 0$, alors $\mathscr{L}$ n'a pas de section strictement effective non-nulle;

Démonstration. - 1) Comme $\widehat{\mu}_{\max }^{\pi}\left(\bar{\theta}_{\mathscr{X}}\right)=0$, d'après la proposition 4.2.10 1), on a $\widehat{\mu}_{\max }^{\pi}(\overline{\mathscr{L}})+\widehat{\mu}_{\max }^{\pi}\left(\overline{\mathscr{L}}^{\vee}\right) \leqslant 0$. Donc $\widehat{\mu}_{\max }^{\pi}(\overline{\mathscr{L}})>0$ implique $\widehat{\mu}_{\max }^{\pi}\left(\overline{\mathscr{L}}^{\vee}\right)<0$.

2) Si $f: \mathscr{\theta}_{\mathscr{X}} \rightarrow \mathscr{L}$ est un homomorphisme non-nul correspondant à une section effective de $\overline{\mathcal{L}}$, alors on a $h(f) \leqslant 0$. D'après $(76)$, on a $\widehat{\mu}_{\max }^{\pi}(\overline{\mathscr{L}}) \geqslant \widehat{\mu}_{\max }^{\pi}\left(\overline{\mathscr{\vartheta}}_{\mathscr{X}}\right)=0$.

3) est similaire à 2).

Lemme 4.2.15. - Soient $\overline{\mathscr{L}}$ et $\overline{\mathscr{L}}$ deux fibrés inversibles hermitiens sur $\mathscr{X}$. On suppose que $\mathscr{L}_{K}$ est gros et qu'une puissance tensorielle de $\overline{\mathscr{L}}$ a une section strictement effective non-nulle. Pour tout $a \in \mathbb{R}$, il existe un entier $n \geqslant 1$ et un homomorphisme injectif $\varphi: \mathscr{L} \rightarrow \mathscr{L}^{\otimes n}$ tel que $h_{\sigma}(\varphi)<$ a quel que soit $\sigma: K \rightarrow \mathbb{C}$.

Démonstration. - On suppose que $\overline{\mathscr{L}}^{\otimes m}$ a une section strictement effective non-nulle qui correspond à un homomorphisme $\eta: \theta_{\mathscr{X}} \rightarrow \mathscr{L}^{\otimes m}$ tel que $h_{\sigma}(\eta)<0$ quel que soit $\sigma: K \rightarrow \mathbb{C}$. Comme $\mathscr{L}$ est gros, il existe un entier $p \geqslant 1$ ainsi qu'un homomorphisme injectif $\mathscr{L}_{K} \rightarrow \mathscr{L}_{K}^{\otimes p}$ qui induit un homomorphisme injectif $f: \mathscr{L} \rightarrow \mathscr{L}^{\otimes p}$ (voir la proposition 1.4.3 et le lemme 4.2.5). On choisit un entier $r$ tel que $r h_{\sigma}(\eta)+h_{\sigma}(f)<a$ pour tout $\sigma$. D'après la remarque 4.2.7 (3), l'homomorphisme $\varphi=\eta^{\otimes r} \otimes f: \mathcal{L} \rightarrow$ $\mathscr{L}^{\otimes(p r+m)}$ vérifie l'inégalité $h_{\sigma}(\varphi)<a$ pour tout $\sigma: K \rightarrow \mathbb{C}$.

Remarque 4.2.16. - 1) Suivant la terminologie de Moriwaki [37, §2], si un fibré inversible hermitien $\overline{\mathscr{L}}$ est tel que $\mathscr{L}_{K}$ soit gros et qu'une puissance tensorielle positive de $\overline{\mathscr{L}}$ admette une section strictement effective non-nulle, alors $\overline{\mathscr{L}}$ est dit arithmétiquement gros.

2) Soit $\mathscr{L}$ un fibré inversible sur $\mathscr{X}$ tel que $\mathscr{L}_{K}$ soit gros. Il existe toujours un fibré inversible hermitien ayant $\mathscr{L}$ comme fibré inversible sous-jacent et dont une puissance tensorielle admette une section strictement effective non-nulle. En effet, soit $\overline{\mathscr{L}}$ un fibré inversible hermitien dont le fibré inversible sous-jacent est $\mathscr{L}$. Comme $\mathscr{L}_{K}$ est gros, il existe une puissance tensorielle $\mathscr{L}_{K}^{\otimes p}$ qui admet une section globale non-nulle. D'après le lemme 4.2 .5 , il existe un homomorphisme injectif $\varphi: \theta_{\mathscr{X}} \rightarrow \mathscr{L}^{\otimes p}\left(p \in \mathbb{N}_{*}\right)$. D'après la remarque 4.2.7 (3), (4) et (6), quitte à tordre $\overline{\mathscr{L}}$ par $\overline{\vartheta_{\mathscr{X}}(a)}$ avec $a$ assez positif on obtient un fibré inversible hermitien ayant $\mathscr{L}$ comme fibré inversible sous-jacent et dont la $p^{\text {ème }}$ puissance admet une section strictement effective non-nulle. 
LEMME 4.2.17. - Soient $\overline{\mathscr{L}}$ un fibré inversible hermitien arithmétiquement gros et $\overline{\mathcal{E}}$ un fibré vectoriel normé sur $\mathscr{X}$. Pour tout nombre a $\in \mathbb{R}$, il existe deux entiers $m, n \geqslant 1$ et un homomorphisme injectif $\varphi: \mathcal{E} \rightarrow\left(\mathscr{L}^{\otimes n}\right)^{\oplus m}$ tel que $h_{\sigma}(\varphi)<$ a quel que soit $\sigma: K \rightarrow \mathbb{C}$.

Démonstration. - Comme le fibré inversible hermitien $\overline{\mathscr{L}}$ est arithmétiquement gros, $\mathscr{L}_{K}$ est gros. Donc il existe deux entiers $p, m \geqslant 1$ et un homomorphisme injectif $\mathscr{E}_{K} \rightarrow\left(\mathscr{L}_{K}^{\otimes p}\right)^{\oplus m}$ (cf. proposition 1.4.3 infra), qui induit un homomorphisme injectif $\psi: \mathscr{E} \rightarrow\left(\mathscr{L}^{\otimes p}\right)^{\oplus m}$, compte tenu du lemme 4.2.5. D'après le lemme 4.2.15, il existe un entier $q \geqslant 1$ et un homomorphisme $\eta: \Theta_{\mathscr{X}} \rightarrow \mathscr{L}^{\otimes q}$ tel que $h_{\sigma}(\eta)<a-h_{\sigma}(\psi)$ quel que soit $\sigma: K \rightarrow \mathbb{C}$. On note $\varphi=\psi \otimes \eta$. D'après la remarque $4.2 .7(3)$, on a, pour tout $\sigma, h_{\sigma}(\varphi)<a$.

DÉFInITION 4.2.18. - Soit $\overline{\mathscr{E}}$ un fibré inversible hermitien sur $\mathscr{X}$. On dit que $\overline{\mathscr{E}}$ est faiblement positif si, pour tout fibré inversible hermitien $\overline{\mathscr{L}} \operatorname{sur} \mathscr{X}$, il existe $\lambda>0$ tel que, pour tout entier $D>\lambda$ et tout entier $n>\lambda D$, le fibré inversible hermitien $\overline{\mathcal{E}}^{\vee \otimes n} \otimes \overline{\mathcal{L}}^{\otimes D}$ n'a pas de section effective non-nulle.

Lemme 4.2.19. - Soient $\overline{\mathcal{E}}_{1}$ et $\overline{\mathcal{E}}_{2}$ deux fibrés vectoriels normés sur $\mathscr{X}, f: \mathcal{E}_{1} \rightarrow \mathcal{E}_{2}$ un homomorphisme injectif et tel que $h_{\sigma}(f) \leqslant 0$ quel que soit le plongement $\sigma$ : $K \rightarrow \mathbb{C}$. Si le fibré vectoriel normé $\bar{\delta}_{2}$ n'a pas de section effective (resp. strictement effective) non-nulle, alors il en est de même de $\overline{\mathscr{E}}_{1}$.

Démonstration. - Si $\varphi: \Theta_{\mathscr{X}} \rightarrow \mathcal{E}_{1}$ est un homomorphisme non-nul qui correspond à une section effective (resp. strictement effective), alors l'homomorphisme composé $f \varphi$ correspond à une section effective (resp. strictement effective) non-nulle de $\overline{\mathscr{E}}_{2}$ (voir la remarque $4.2 .7(5))$.

Dans la suite, on présente quelques formes équivalentes de la condition de positivité faible.

Proposition 4.2.20. - Soit $\overline{\mathcal{E}}$ un fibré inversible hermitien sur $\mathscr{X}$. Les conditions suivantes sont équivalentes:

1) pour tout fibré inversible hermitien $\overline{\mathscr{L}}$ et tout fibré vectoriel normé $\overline{\mathcal{F}}$ sur $\mathscr{X}$, il existe $\lambda>0$ tel que, pour tout entier $D>\lambda$ et tout entier $n>\lambda D, \overline{\mathcal{E}}^{\vee \otimes n} \otimes \overline{\mathscr{L}}^{\otimes D} \otimes \overline{\mathcal{F}}$ n'a pas de section effective non-nulle;

2) $\bar{\varepsilon}$ est faiblement positif;

3) il existe un fibré inversible hermitien $\overline{\mathscr{L}}$ sur $\mathscr{X}$ qui est arithmétiquement gros au sens de Moriwaki, et un nombre $\lambda>0$ tel que, pour tout entier $D>\lambda$ et tout entier $n>\lambda D, \overline{\mathcal{E}}^{\vee \otimes n} \otimes \overline{\mathscr{L}}^{\otimes D}$ n'a pas de section effective non-nulle. 
Démonstration. - "1) $\Longrightarrow 2) \Longrightarrow 3$ )" sont triviaux.

"3) $\Longrightarrow 1$ )": D'après les lemmes 4.2 .15 et 4.2 .17 , il existe trois entiers $p, q, r \geqslant 1$ ainsi que deux homomorphismes injectifs $\varphi: \mathcal{F} \rightarrow\left(\mathscr{L}^{\otimes p}\right)^{\oplus r}$ et $\psi: \mathscr{L} \rightarrow \mathscr{L}^{\otimes q}$ tels que $h_{\sigma}(\varphi)<0$ et $h_{\sigma}(\psi)<0$ quel que soit $\sigma: K \rightarrow \mathbb{C}$. Pour tout entier $D \geqslant 1$, l'homomorphisme $\phi_{D}=\psi^{\otimes D} \otimes \varphi$ de $\mathscr{L}^{\otimes D} \otimes \mathcal{F}$ vers $\left(\mathscr{L}^{\otimes(D q+p)}\right)^{\oplus r}$ est injectif. De plus, d'après la remarque 4.2.7 $(3)$, on a $h_{\sigma}\left(\phi_{D}\right)<0$ pour tout plongement $\sigma: K \rightarrow \mathbb{C}$. La condition 3) prédit qu'il existe $\lambda>0$ tel que, pour tout entier $D>\lambda$ et tout entier $n>\lambda D, \bar{E}^{\vee \otimes n} \otimes \overline{\mathscr{L}}^{\otimes(D q+p)}$ n'a pas de section effective non-nulle. Par conséquent, pour tel $D$ et tel $n, \overline{\mathscr{E}}^{\vee \otimes n} \otimes \overline{\mathcal{L}}^{\otimes D} \otimes \overline{\mathcal{F}}$ n'a pas de section effective non-nulle, compte tenu du lemme 4.2.19 et de la remarque 4.2 .7 (2).

Dans le théorème qui suit est présenté un critère de la condition de positivité faible par la négativité de la pente maximale asymptotique du dual du fibré inversible hermitien. Lorsque la fibre générique du fibré est négative, c'est-à-dire que la positivité verticale est exclue, alors ces deux conditions sont équivalentes.

THÉORÈme 4.2.21. - Soit $\overline{\mathscr{L}}$ un $\bigoplus_{\mathscr{X}}$-module inversible hermitien. Si $\widehat{\mu}_{\max }^{\pi}\left(\overline{\mathscr{L}}^{\vee}\right)<0$, alors $\overline{\mathscr{L}}$ est faiblement positif. La réciproque est vraie lorsque $\mathscr{L}_{K}^{\vee}$ est gros.

Démonstration. - "Nécessité": Comme $\widehat{\mu}_{\max }^{\pi}\left(\overline{\mathscr{L}}^{\vee}\right)<0$, il existe une constante $\varepsilon>0$ et un fibré inversible hermitien $\overline{\mathscr{L}}$ sur $\mathscr{X}$ tels que $\mathscr{L}_{K}$ soit gros et que $A_{m}\left(\overline{\mathscr{L}}^{\vee}, \overline{\mathscr{L}}\right) \leqslant-\varepsilon$ pour tout entier $m$ suffisamment grand. Pour tout fibré inversible hermitien $\bar{M}$ sur Spec $\theta_{K}$, on a $A_{m}\left(\overline{\mathscr{L}}^{\vee}, \overline{\mathscr{L}}\right)=A_{m}\left(\overline{\mathscr{L}}^{\vee}, \overline{\mathscr{L}} \otimes \pi^{*} \bar{M}\right)$. En particulier, pour tout $a \in \mathbb{R}$

$$
A_{m}\left(\overline{\mathscr{L}}^{\vee}, \overline{\mathscr{L}}\right)=A_{m}\left(\overline{\mathscr{L}}^{\vee}, \overline{\mathscr{L}} \otimes \pi^{*} \overline{\vartheta_{K}(a)}\right)=A_{m}\left(\overline{\mathscr{L}}^{\vee}, \overline{\mathscr{L}} \otimes \overline{\vartheta_{\mathscr{X}}(a)}\right),
$$

où $\overline{\theta_{K}(a)}$ désigne le fibré inversible hermitien sur $\operatorname{Spec} \theta_{K}$ dont le $\theta_{K}$-module sousjacent est trivial et tel que $\|\mathbf{1}\|_{\sigma}=e^{-a}$ pour tout $\sigma: K \rightarrow \mathbb{C}, \mathbf{1}$ étant l'élément unité de $\theta_{K}$. Quitte à tordre $\overline{\mathscr{L}}$ par $\overline{\theta_{\mathscr{X}}(a)}$ avec $a$ assez positif, on peut supposer que $\overline{\mathscr{L}}$ est arithmétiquement gros (voir la remarque 4.2.16 2)). D'après la proposition 4.2.8 1), on a $A_{1}\left(\overline{\mathscr{L}}^{\vee}, \overline{\mathscr{L}}\right) \leqslant A_{m}\left(\overline{\mathscr{L}}^{\vee}, \overline{\mathscr{L}}\right) \leqslant-\varepsilon$ et donc $\widehat{\mu}_{\text {max }}^{\pi}\left(\overline{\mathscr{L}}^{\vee} \otimes \overline{\mathscr{L}}\right) \leqslant \widehat{\mu}_{\text {max }}^{\pi}(\overline{\mathscr{L}})-\varepsilon$. Soit $\lambda>\varepsilon^{-1} \widehat{\mu}_{\max }^{\pi}(\overline{\mathscr{L}})$ une constante. Pour tout entier $D \geqslant 1$ et tout entier $n>\lambda D$, on a, d'après la proposition 4.2.10 1) et 2 ),

$$
(n-D) \widehat{\mu}_{\max }^{\pi}(\overline{\mathscr{L}})+\widehat{\mu}_{\max }^{\pi}\left(\overline{\mathscr{L}}^{\vee \otimes n} \otimes \overline{\mathscr{L}}^{\otimes D}\right) \leqslant \widehat{\mu}_{\max }^{\pi}\left(\left(\overline{\mathscr{L}}^{\vee} \otimes \overline{\mathscr{L}}\right)^{\otimes n}\right) \leqslant n\left(\widehat{\mu}_{\max }^{\pi}(\overline{\mathscr{L}})-\varepsilon\right) .
$$

Par conséquent,

$$
\widehat{\mu}_{\max }^{\pi}\left(\overline{\mathscr{L}}^{\vee \otimes n} \otimes \overline{\mathscr{L}}^{\otimes D}\right) \leqslant D \widehat{\mu}_{\max }^{\pi}(\overline{\mathscr{L}})-n \varepsilon<0 .
$$

Donc $\overline{\mathscr{L}}^{\vee \otimes n} \otimes \overline{\mathscr{L}}^{\otimes D}$ n'a pas de section effective non-nulle, compte tenu de la proposition 4.2 .142 ). D'après la proposition $4.2 .20, \overline{\mathcal{L}}$ est faiblement positif.

"Suffisance": Soit $\bar{M}$ un fibré inversible hermitien sur $\operatorname{Spec} \theta_{K}$ tel que $\widehat{\mu}(\bar{M})>0$. D'après la proposition $4.2 .20(2) \Rightarrow 1$ )) Il existe une constante $\lambda>0$ telle que, pour 
tout entier $D>\lambda$ et tout entier $n>\lambda D$, on ait $-\log \lambda_{1}\left(\pi_{*}\left(\overline{\mathscr{L}}^{\vee \otimes n} \otimes \pi^{*}\left(\bar{M}^{\otimes D}\right)\right)\right) \leqslant 0$. D'après (63) et $(37)$, on a

$$
\widehat{\mu}_{\max }\left(\pi_{*}\left(\overline{\mathscr{L}}^{\vee \otimes n} \otimes \pi^{*}\left(\bar{M}^{\otimes D}\right)\right)\right)=\widehat{\mu}_{\max }\left(\pi_{*}\left(\overline{\mathscr{L}}^{\vee \otimes n}\right)\right)+D \widehat{\mu}(\bar{M}) \leqslant U\left(r_{n}, K\right),
$$

où $r_{n}=\operatorname{rg}\left(\pi_{*}\left(\mathcal{L}^{\vee \otimes n}\right)\right)$. Soit $\left(D_{n}\right)_{n \geqslant 1}$ une suite telle que

i) $D_{n}>\lambda$ pour tout entier $n \geqslant 1$;

ii) $D_{n}<n / \lambda$ pour $n$ suffisamment grand;

iii) $\lim _{n \rightarrow+\infty} n / D_{n}=\lambda$.

D'après (77), pour tout entier $n$ assez grand,

$$
\frac{1}{n} \widehat{\mu}_{\max }\left(\pi_{*}\left(\overline{\mathscr{L}}^{\vee \otimes n}\right)\right)+\frac{D_{n}}{n} \widehat{\mu}(\bar{M}) \leqslant \frac{1}{n} U\left(r_{n}, K\right) .
$$

Par passage à la limite on obtient $\widehat{\mu}_{\max }^{\pi}\left(\overline{\mathscr{L}}^{\vee}\right) \leqslant-\lambda^{-1} \widehat{\mu}(\bar{M})<0$, en s'appuyant sur l'estimation $U(r, K) \ll \log (r+1)$, établie dans le lemme 4.2.1.

La condition de positivité faible implique (est donc équivalente à) une condition de croissance exponentielle de la norme du plus petit vecteur non-nul.

Proposition 4.2.22. - Soient $\overline{\mathscr{L}}$ et $\overline{\mathscr{L}}$ deux fibrés inversibles hermitiens sur $\mathscr{X}$. Si $\overline{\mathcal{L}}$ est faiblement positif, alors il existe deux nombres réels strictement positifs a et $\lambda^{\prime}$ tels que, pour tout entier $D>\lambda^{\prime}$ et tout entier $n>\lambda^{\prime} D$, on ait $\lambda_{1}\left(\pi_{*}\left(\overline{\mathscr{L}}^{\vee \otimes n} \otimes\right.\right.$ $\left.\left.\overline{\mathscr{L}}^{\otimes D}\right)\right) \geqslant e^{a n}$.

Démonstration. - D'après le lemme 4.2.15, il suffit de démontrer la proposition pour le cas où $\overline{\mathscr{L}}$ et $\overline{\mathscr{L}} \otimes \overline{\mathscr{L}}$ admettent au moins une section effective non-nulle. En effet, si $f: \overline{\mathscr{L}} \rightarrow \overline{\mathscr{L}}^{\prime}$ est un homomorphisme injectif tel que $h_{\sigma}(f) \leqslant 0$ pour tout $\sigma$, alors $\lambda_{1}\left(\overline{\mathscr{L}}^{\vee \otimes n} \otimes \overline{\mathscr{L}}^{\otimes D}\right) \geqslant \lambda_{1}\left(\overline{\mathscr{L}}^{\vee \otimes n} \otimes \overline{\mathscr{L}}^{\prime \otimes D}\right)$ (cf. la remarque 4.2 .7 (7)). Soit $\bar{M}$ un fibré inversible hermitien sur Spec $\theta_{K}$ dont le $\theta_{K}$-module inversible sous-jacent $M$ est trivial, et tel que, pour tout $\sigma: K \rightarrow \mathbb{C}$, on ait $\|\mathbf{1}\|_{\sigma}=e^{-b}$, où $b>0, \mathbf{1}$ est l'élément d'unité de $M \cong \theta_{K}$. On a $\widehat{\mu}(\bar{M})=b$, et $\pi^{*}(\bar{M})=\overline{\vartheta_{\mathscr{X}}(b)}$. Comme $\overline{\mathcal{L}}$ est faiblement positif, il existe $\lambda>0$ tel que, pour tout entier $D>\lambda$ et tout entier $n>\lambda D$, on ait

$$
\lambda_{1}\left(\pi_{*}\left(\overline{\mathscr{L}}^{\vee \otimes n} \otimes \overline{\mathscr{L}}^{\otimes D} \otimes \pi^{*}(\bar{M})^{\otimes D}\right)\right)>1,
$$

ou encore $\lambda_{1}\left(\pi_{*}\left(\overline{\mathscr{L}}^{\vee \otimes n} \otimes \overline{\mathscr{L}}^{\otimes D}\right)>e^{b D}\right.$, compte tenu de la remarque 4.2.7 (4) et (7). On fixe deux entiers $D_{0}$ et $n_{0}$ tels que $D_{0}>\lambda$ et $n_{0}>\lambda D_{0}$. Pour tout entier $n>0$, on a

$$
\lambda_{1}\left(\pi_{*}\left(\overline{\mathscr{L}}^{\vee \otimes n n_{0}} \otimes \overline{\mathscr{L}}^{\otimes n D_{0}}\right)>e^{b n D_{0}} .\right.
$$

Comme $\overline{\mathscr{L}}$ a une section effective, et comme il existe un homomorphisme $\phi$ de $\overline{\mathscr{L}}^{\vee}$ vers $\overline{\mathscr{L}}$ tel que $h_{\sigma}(\phi) \leqslant 0$ pour tout $\sigma$, d'après la remarque $4.2 .7(7)$, on obtient

$$
\lambda_{1}\left(\pi_{*}\left(\overline{\mathscr{L}}^{\vee \otimes\left(n n_{0}+l\right)} \otimes \overline{\mathscr{L}}^{\otimes D}\right)\right) \geqslant \lambda_{1}\left(\pi_{*}\left(\overline{\mathscr{L}}^{\vee \otimes n n_{0}} \otimes \overline{\mathscr{L}}^{\otimes n D_{0}}\right)>e^{b n D_{0}}\right.
$$


pour tous les entiers $D$ et $l$ tels que $D_{0} \leqslant D \leqslant n D_{0}$ et $0 \leqslant l \leqslant n D_{0}-D$. On note

$$
\lambda_{0}=\frac{n_{0}^{2}}{D_{0}^{2}}+\frac{2 n_{0}}{D_{0}}
$$

Pour tout entier $D \geqslant D_{0}$ et tout entier $m \geqslant \lambda_{0} D$, si on pose $n=\left\lfloor m / n_{0}\right\rfloor$ et $l=$ $m-n n_{0}$, alors on a $m=n n_{0}+l$, où $0 \leqslant l<n_{0}$. En outre, on a

$$
\begin{aligned}
n D_{0}-D & \geqslant\left(m / n_{0}-1\right) D_{0}-D \geqslant\left(\lambda_{0} D / n_{0}-1\right) D_{0}-D \\
& \geqslant n_{0} D / D_{0}+2 D-D_{0}-D \geqslant n_{0} .
\end{aligned}
$$

Donc $l<n_{0} \leqslant n D_{0}-D$ et $D \leqslant n D_{0}$. Par conséquent,

$$
\lambda_{1}\left(\pi_{*}\left(\overline{\mathscr{L}}^{\vee \otimes m} \otimes \overline{\mathscr{L}}^{\otimes D}\right)\right) \geqslant e^{b D_{0} m / 2 n_{0}}
$$

puisque $n \geqslant m / 2 n_{0}$. En prenant $a=b D_{0} / 2 n_{0}$, on achève la démonstration.

La proposition 4.2.22 suggère la généralisation suivante de la condition de positivité faible pour un fibré vectoriel hermitien.

DÉfinition 4.2.23. - Soit $\bar{E}$ un fibré vectoriel hermitien sur $\mathscr{X}$. On dit que $\bar{E}$ est faiblement positif si, pour tout fibré inversible hermitien $\overline{\mathcal{L}}$ sur $\mathscr{X}$, il existe deux nombres réels strictement positifs $a$ et $\lambda$ tels que, pour tout entier $D>\lambda$ et tout entier $n \geqslant \lambda D$, on ait $\lambda_{1}\left(\pi_{*}\left(S^{n} \overline{\mathcal{E}}^{\vee} \otimes \overline{\mathcal{L}}^{\otimes D}\right)\right)>e^{a n}$.

REmarque 4.2.24. - On peut proposer une autre condition en faisant une généralisation naïve de la définition 4.2 .18 au cas de fibré vectoriel hermitien. Certainement cette condition est plus faible que celle dans la définition 4.2.23. Mais il n'est pas claire, au moins pour l'auteur, que l'analogue de la proposition 4.2.22 est encore valable pour le cas de rang $>1$.

La proposition suivante est un analogue au cas de rang supérieur de la proposition 4.2.20. Sa démonstration est presque la même.

Proposition 4.2.25. - Les conditions suivantes sont équivalentes:

1) pour tout fibré inversible hermitien $\overline{\mathscr{L}}$ et tout fibré vectoriel normé $\overline{\mathcal{F}}$ sur $\mathscr{X}$ il existe deux nombres réels a, $\lambda>0$ tels que, pour tout entier $D>\lambda$ et tout entier $n \geqslant \lambda D$, on ait

$$
\lambda_{1}\left(\pi_{*}\left(S^{n} \overline{\mathcal{E}}^{\vee} \otimes \overline{\mathcal{L}}^{\otimes D} \otimes \overline{\mathcal{F}}\right)\right)>e^{a n}
$$

2) $\bar{\varepsilon}$ est faiblement positif;

3) il existe un fibré inversible hermitien arithmétiquement gros $\overline{\mathscr{L}}$ sur $\mathscr{X}$ et deux nombres $a, \lambda>0$ tels que, pour tout entier $D>\lambda$ et tout entier $n \geqslant \lambda D$, on ait

$$
\lambda_{1}\left(\pi_{*}\left(S^{n} \overline{\mathcal{E}}^{\vee} \otimes \overline{\mathscr{L}}^{\otimes D}\right)\right)>e^{a n} .
$$

La proposition 4.2.27 au-dessous montre que la condition de positivité faible d'un fibré vectoriel hermitien $\bar{E}$ est équivalente à la même condition du dual du fibré canonique de $\mathbb{P}\left(\mathcal{E}^{\vee}\right)$ muni des métriques de Fubini-Study. Ce résultat nous permet de ramener l'étude de cette condition au cas de fibré inversible hermitien. 
Lemme 4.2.26. - Soit $(V,\|\cdot\|)$ un espace hermitien de dimension $r>0$. Soit $\bar{L}$ le fibré inversible $\Theta_{V}(1)$ sur $\mathbb{P}(V)$, muni de la métrique de Fubini-Study. Pour tout entier $n \geqslant 1$, on désigne respectivement $\|\cdot\|_{\text {sup }}$ et $\|\cdot\|_{\text {sym }}$ la norme sup et la norme puissance symétrique sur $H^{0}\left(\mathbb{P}(V), L^{\otimes n}\right) \cong S^{n} V$. Alors, pour toute section non-nulle $s \in H^{0}\left(\mathbb{P}(V), L^{\otimes n}\right)$, on $a$

$$
\left|\log \|s\|_{\text {sup }}-\log \|s\|_{\text {sym }}\right| \leqslant \frac{1}{2} \log C_{1}(n, r),
$$

où $C_{1}(n, r)=\left(\begin{array}{c}n+r-1 \\ n\end{array}\right)$ est le rang de $S^{n} V$.

Démonstration. - On désigne par $\|\cdot\|_{\mathrm{J}}$ la métrique de John associée à la métrique $\|\cdot\|_{\text {sup }}$. Comme $\|\cdot\|_{\text {sup }}$ est invariante par l'action du groupe unitaire de $V$, il en est de même de la métrique hermitienne $\|\cdot\|_{\mathrm{J}}$. En vertu du [10, lemme 4.3.6], les métriques $\|\cdot\|_{\mathrm{J}}$ et $\|\cdot\|_{\mathrm{sym}}$ sont proportionnelles. Il existe donc un nombre réel $c_{0}(n, r)$ tel que

$$
\forall 0 \neq s \in H^{0}\left(\mathbb{P}(V), L^{\otimes n}\right), \quad \log \|s\|_{\mathrm{J}}=\log \|s\|_{\mathrm{sym}}+c_{0}(n, r) .
$$

Or, si $u$ est un élément dans $H^{0}(\mathbb{P}(V), L)=V$, alors $\left\|u^{n}\right\|_{\text {sup }}=\|u\|^{n}=\left\|u^{n}\right\|_{\text {sym }}$. On en déduit donc $0 \leqslant c_{0}(n, r) \leqslant \frac{1}{2} \log C_{1}(n, r)$, compte tenu de la relation

$$
\|\cdot\|_{\text {sup }} \leqslant\|\cdot\|_{\mathrm{J}} \leqslant \sqrt{C_{1}(n, r)}\|\cdot\|_{\text {sup }} .
$$

La démonstration est donc achevée.

Proposition 4.2.27. - Soit $\overline{\mathcal{E}}$ un fibré vectoriel hermitien de rang $r$ sur $\mathscr{X}$. On désigne par $\overline{\mathcal{L}}$ le fibré inversible $\Theta_{\mathcal{E}^{\vee}}(-1)$ sur $\mathbb{P}\left(\mathcal{E}^{\vee}\right)$, muni des métriques duales des métriques de Fubini-Study sur $\Theta_{\mathscr{E}^{\vee}}(1)$. Alors $\overline{\mathcal{E}}$ est faiblement positif sur $\mathscr{X}$ si et seulement si $\overline{\mathcal{L}}$ est faiblement positif sur $\mathbb{P}\left(\mathcal{E}^{\vee}\right)$.

Démonstration. - Soient $p: \mathbb{P}\left(\mathcal{E}^{\vee}\right) \rightarrow \mathscr{X}$ le morphisme canonique et $r=\operatorname{rg} \mathcal{E}$.

"£": On suppose que $\bar{M}$ est un $\bigoplus_{\mathscr{X}}$-module inversible hermitien. Comme $\overline{\mathcal{L}}$ est faiblement positif, il existe deux nombres $a, \lambda>0$ tels que, pour tout entier $D>\lambda$ et tout entier $n>\lambda D$, on ait

$$
\lambda_{1}\left((\pi p)_{*}\left(\overline{\mathcal{L}}^{\vee \otimes n} \otimes p^{*}(\bar{M})^{\otimes D}\right)>e^{a n} .\right.
$$

D'après le lemme 4.2.26 et la remarque $4.2 .7(7)$, on a

$$
\lambda_{1}\left(\pi_{*}\left(S^{n} \overline{\mathcal{E}}^{\vee} \otimes \bar{M}^{\otimes D}\right)\right) \geqslant C_{1}(n, r)^{-\frac{1}{2}} \lambda_{1}\left((\pi p)_{*}\left(\overline{\mathcal{L}}^{\vee \otimes n} \otimes p^{*}(\bar{M})^{\otimes D}\right)>e^{a n} C_{1}(n, r)^{-\frac{1}{2}},\right.
$$

où $C_{1}(n, r)=\left(\begin{array}{c}n+r-1 \\ n\end{array}\right)$. Comme la fonction $C_{1}(n, r)$ croît polynomialement par rapport à $n$, on obtient le résultat.

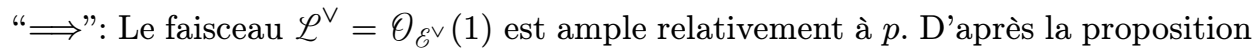
1.4.1, il existe un fibré inversible $M$ sur $\mathscr{X}$ tel que $\mathscr{L}:=\mathscr{L}^{\vee} \otimes p^{*} M$ soit ample. On munit $M$ des métriques continues arbitraires et on pose

$$
\overline{\mathscr{L}}=\overline{\mathscr{L}}^{\vee} \otimes p^{*}\left(\bar{M} \otimes \overline{\bar{\Theta}_{\mathscr{X}}(b)}\right) \cong \overline{\mathcal{L}}^{\vee} \otimes p^{*}(\bar{M}) \otimes \overline{\vartheta_{\mathbb{P}\left(\mathscr{E}^{\vee}\right)}(b)},
$$


où $b$ est un nombre suffisamment positif de sorte que certaine puissance tensorielle de $\overline{\mathscr{L}}$ ait une section strictement effective non-nulle. Donc $\overline{\mathscr{L}}$ est gros (voir la remarque 4.2.16 1)) Dans la suite, on note $\overline{M(b)}=\bar{M} \otimes \overline{\vartheta_{\mathscr{X}}(b)}$.

Comme $\bar{E}$ est faiblement positif, il existe deux nombres réels $a, \lambda>0$ tels que, pour tout entier $D>\lambda$ et tout entier $n>\lambda D$, on ait

$$
\lambda_{1}\left(\pi_{*}\left(S^{n} \overline{\mathcal{E}}^{\vee} \otimes \overline{M(b)}^{\otimes D}\right)\right)>e^{a n} .
$$

D'après le lemme 4.2.26, on obtient

$$
\lambda_{1}\left((\pi p)_{*}\left(\overline{\mathscr{L}}^{\vee \otimes n} \otimes p^{*}(\overline{M(b)})^{\otimes D}\right)\right)=\lambda_{1}\left((\pi p)_{*}\left(\overline{\mathscr{L}}^{\vee \otimes(n-D)} \otimes \overline{\mathscr{L}}^{\otimes D}\right)\right)>e^{a n} C_{1}(n, r)^{-\frac{1}{2}} .
$$

Soit $n_{0} \in \mathbb{N}$ tel que, pour tout $n>n_{0}$, on ait $e^{a n} C_{1}(n, r)^{-\frac{1}{2}}>1$. Soit $\lambda^{\prime}=\max \left(n_{0}, \lambda\right)$. Alors pour tout entier $D>\lambda^{\prime}$ et $n>\lambda^{\prime} D$ on a

$$
\lambda_{1}\left((\pi p)_{*}\left(\overline{\mathscr{L}}^{\vee \otimes n} \otimes \overline{\mathscr{L}}^{\otimes D}\right)\right)>1 .
$$

Donc $\overline{\mathcal{L}}$ est faiblement positif, compte tenu de la proposition $4.2 .20(3) \Rightarrow 2)$ ).

Le théorème suivant donne un critère numérique de la condition de positivité faible, qui généralise le théorème 4.2.21 au cas de rang supérieur.

THÉORÈme 4.2.28. - Soit $\overline{\mathcal{E}}$ un fibré vectoriel hermitien sur $\mathscr{X}$. Si $\widehat{\mu}_{\max }^{\pi}\left(\overline{\mathcal{E}}^{\vee}\right)<0$, alors $\overline{\mathcal{E}}$ est faiblement positif. La réciproque est vraie lorsque $\mathcal{E}_{K}^{\vee}$ est gros.

Démonstration. - Soient $p: \mathbb{P}\left(\mathcal{E}^{\vee}\right) \rightarrow \mathscr{X}$ le morphisme canonique et $\overline{\mathcal{L}}$ le fibré inversible $\theta_{\mathcal{E}^{\vee}}(-1)$ muni des métriques duales à celles de Fubini-Study sur $\theta_{\mathscr{E}^{\vee}}(1)$.

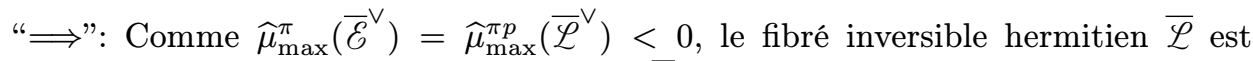
faiblement positif, donc il en est de même de $\overline{\mathcal{E}}$, compte tenu de la proposition 4.2.27.

"£": D'après la proposition 4.2.27, si $\overline{\mathcal{E}}$ est faiblement positif, il en est de même de $\overline{\mathcal{L}}$. D'autre part, si $\mathcal{E}_{K}^{V}$ est gros, $\mathscr{L}_{K}^{\vee}$ est gros. D'après le théorème 4.2.21, on obtient

$$
\widehat{\mu}_{\max }^{\pi}\left(\bar{\varepsilon}^{\vee}\right)=\widehat{\mu}_{\max }^{\pi p}\left(\overline{\mathscr{L}}^{\vee}\right)<0 .
$$

\subsection{Polygones et pentes asymptotiques en géométrie relative}

Dans cette section, on applique les résultats obtenus dans le chapitre 3 à l'étude du comportement asymptotique des fibrés vectoriels dans le cadre de la géométrique relative.

On fixe un corps $k$ et une courbe projective et régulière $C \operatorname{sur} \operatorname{Spec} k$. On désigne par $K^{\prime}=k(C)$ le corps des fonctions rationnelles sur $C$. Soit $g$ le genre de $C$. Pour tout fibré vectoriel $E$ sur $C$, le théorème de Riemann-Roch implique que

$$
\chi(E)=\operatorname{rg} H^{0}(C, E)-\operatorname{rg} H^{1}(C, E)=\operatorname{deg}(E)+\operatorname{rg}(E)(1-g) .
$$


4.3.1. Polygone et pentes asymptotiques. - Soit $B=\bigoplus_{n \geqslant 0} B_{n}$ une $\theta_{C^{-}}$-algèbre graduée. On suppose que chaque $B_{n}$ est un $\theta_{C}$-module localement libre de rang fini et que $B_{n} \neq 0$ pour $n$ suffisamment grand. De plus, on suppose que $B_{K^{\prime}}$ est un anneau intègre. Dans ce sous-paragraphe, on montre que la suite de polygones $\left(\mathscr{P}_{B_{n}} / n\right)_{n \geqslant 1}$ converge uniformément, où les polygones $\mathscr{P}_{B_{n}}$ sont définis dans $§ 2.3 .2$. C'est un analogue dans le cadre de la géométrie relative du théorème 4.1.2. On rappelle d'abord une estimation de la pente minimale de produit tensoriel établie dans [17].

Soit $b(C)=\min \{\operatorname{deg}(H) \mid H \in \operatorname{Pic}(C), H$ est ample $\}$. C'est un entier strictement positif, et l'ensemble des valeurs $\operatorname{de} \operatorname{deg}(H)$ lorsque $H$ décrit $\operatorname{Pic}(C)$ est exactement $b(C) \mathbb{Z}$. En effet, un faisceau inversible $H \in \operatorname{Pic}(C)$ est ample si et seulement si $\operatorname{deg}(H)>0$. Soit en outre $a(C)=b(C)+g-1$.

Lemme 4.3.1. - Soient $E$ et $F$ deux fibrés vectoriels sur $C$. On a $\mu_{\min }(E \otimes F) \geqslant$ $\mu_{\min }(E)+\mu_{\min }(F)-a(C)$.

Démonstration. - Il suffit d'appliquer [17] Proposition 2.2 aux fibrés vectoriels $E^{\vee}$ et $F^{\vee}$. En utilisant le fait que $\mu_{\max }\left(E^{\vee}\right)=-\mu_{\min }(E)$, on obtient le résultat.

ThÉORÈme 4.3.2. - Soit $B=\bigoplus_{n \geqslant 0} B_{n}$ une algèbre graduée en fibrés vectoriels sur $C$. On suppose que $B_{K^{\prime}}$ est intègre, $B_{n} \neq 0$ pour $n$ suffisamment grand et qu'il existe $\alpha>0$ tel que $\mu_{\max }\left(B_{n}\right) \leqslant \alpha n$ quel que soit $n \geqslant 1$.

1) La suite de pentes maximales $\left(\mu_{\max }\left(B_{n}\right) / n\right)_{n \geqslant 1}$ converge dans $\mathbb{R}$.

2) Si l'algèbre $B_{K^{\prime}}$ est de type fini sur $K^{\prime}$, la suite de polygones $\left(\mathscr{P}_{B_{n}} / n\right)_{n \geqslant 1}$ converge uniformément vers une fonction concave sur $[0,1]$.

3) $S i$, pour tous entiers $n$ et $m$ assez grands, l'homomorphisme $B_{n, K^{\prime}} \otimes B_{m, K^{\prime}} \rightarrow$ $B_{n+m, K^{\prime}}$ induit par la structure d'algèbre de $B$ est surjectif, alors la suite de pentes minimales $\left(\mu_{\min }\left(B_{n}\right) / n\right)_{n \geqslant 1}$ converge dans $\mathbb{R}$.

Démonstration. - D'après un argument similaire à celui dans la démonstration du théorème 4.1.2, en utilisant le lemme 4.3.1, on obtient que l'algèbre graduée $B_{K^{\prime}}=$ $\bigoplus_{n \geqslant 0} B_{n, K^{\prime}}$, munie des filtrations de Harder-Narasimhan, est $f$-pseudo-filtrée pour la fonction constante $f=\frac{1}{2} a(C)$. Le théorème résulte donc du théorème 3.4.6 et les propositions 3.2 .9 et 3.2.10.

4.3.2. Théorème de Hilbert-Samuel. - Soient $X$ une variété projective sur $k$ et $\pi: X \rightarrow C$ un $k$-morphisme projectif et plat. Soit $d$ la dimension relative de $X$ sur $C$. Soit $L$ un $\theta_{X}$-module inversible. On établit un analogue du théorème 4.1 .8 en géométrie relative. L'idée est d'appliquer le théorème 4.3 .2 à l'algèbre $B=$ $\bigoplus_{n \geqslant 0} \pi_{*}\left(L^{\otimes n}\right)$. Comme $\pi$ est un morphisme plat, $\pi_{*}\left(L^{\otimes n}\right)$ est un $\theta_{C}$-module cohérent sans torsion, donc est un $\theta_{C}$-module localement libre de rang fini puisque $C$ est régulière. On commence par un résultat (proposition 4.3.4) sur la comparaison de la pente maximale au plus grand degré de sous-fibré inversible.

LEMme 4.3.3 ([17] Lemma 2.1). - Soit $E$ un $\theta_{C}$-module localement libre de rang fini et non-nul. Si $H^{0}(C, E)$ est nul, alors $\mu_{\max }(E) \leqslant g-1$. 
Proposition 4.3.4. - Pour tout $\theta_{C}$-module localement libre de rang fini et non-nul $E$, il existe un sous- $\mathscr{\vartheta}_{C}$-module inversible $\mathscr{L}$ tel que $\operatorname{deg}(\mathscr{L}) \geqslant \mu_{\max }(E)-a(C)$, où $a(C)$ est la constante définie dans \$4.3.1.

Démonstration. - Soit $M$ un $\theta_{C}$-module inversible de degré $b(C)$. On choisit $r \in \mathbb{Z}$ tel que

$$
g+b(C)-1 \geqslant \mu_{\max }\left(E \otimes M^{\otimes r}\right)=\mu_{\max }(E)+r b(C)>g-1 .
$$

D'après le lemme 4.3.3 on obtient $H^{0}\left(C, E \otimes M^{\otimes r}\right) \neq 0$. Donc il existe un homomorphisme injectif de $\theta_{C}$ vers $E \otimes M^{\otimes r}$. Soit $\mathcal{L}=M^{\vee \otimes r}$. Il existe alors un homomorphisme injectif de $\mathscr{L}$ vers $E$. En outre, on a

$$
\operatorname{deg}(\mathscr{L})=-r \operatorname{deg}(M)=-r b(C) \geqslant \mu_{\max }(E)-g-b(C)+1 .
$$

Comme $a(C)=b(C)+g-1$, on obtient $\operatorname{deg}(\mathscr{L}) \geqslant \mu_{\max }(E)-a(C)$.

La proposition au-dessous est une estimation sur la croissance des pentes maximales. Elle montre en particulier que l'algèbre $B=\bigoplus_{n \geqslant 0} \pi_{*}\left(L^{\otimes n}\right)$ vérifie la condition du théorème 4.3.2.

Proposition 4.3.5. - Pour tout $\theta_{X}$-module inversible $L$, il existe une constante $\alpha$ dans $\mathbb{R}$ telle que, pour tout entier $n>1$, on ait $\mu_{\max }\left(\pi_{*}\left(L^{\otimes n}\right)\right) \leqslant \alpha n$.

Démonstration. - La variété $X$ étant projective sur $k$, on choisit un $\Theta_{X}$-module inversible ample $\mathscr{L}$ sur $X$. L'égalité $\pi_{*}\left(c_{1}(\mathscr{L})^{d}\right)=c_{1}\left(L_{K^{\prime}}\right)^{d}[C]$ est vraie dans le groupe de Chow $\mathrm{CH}_{1}(C)$. Soient $M$ un sous- $\theta_{C}$-module inversible de $\pi_{*}\left(\mathscr{L}^{\otimes n}\right)$ et $\varphi: M \rightarrow \pi_{*}\left(\mathscr{L}^{\otimes n}\right)$ l'homomorphisme canonique. On désigne par $\widetilde{\varphi}: \pi^{*} M \rightarrow \mathscr{L}^{\otimes n}$ le morphisme obtenu de $\varphi$ par adjonction. Ce dernier s'identifie à une section nonidentiquement nulle de $\pi^{*} M^{\vee} \otimes \mathscr{L}^{\otimes n}$, dont le diviseur [div $\left.(\widetilde{\varphi})\right]$ est effectif. On a donc $\operatorname{deg}_{X}\left(c_{1}(\mathscr{L})^{d}[\operatorname{div}(\widetilde{\varphi})]\right) \geqslant 0$. Or $[\operatorname{div} \widetilde{\varphi}]=-\pi^{*} c_{1}(M)+n c_{1}(L) \operatorname{dans} \mathrm{CH}^{1}(X), \operatorname{donc}$

$$
\begin{aligned}
\operatorname{deg}_{X}\left(c_{1}(\mathscr{L})^{d}[\operatorname{div}(\widetilde{\varphi})]\right) & =\operatorname{deg}_{X}\left(\left(-\pi^{*} c_{1}(M)+n c_{1}(L)\right) c_{1}(\mathscr{L})^{d}\right) \\
& =-\operatorname{deg}_{C}\left(c_{1}(M) \pi_{*}\left(c_{1}(\mathscr{L})^{d}\right)\right)+n \operatorname{deg}_{X}\left(c_{1}(L) c_{1}(\mathscr{L})^{d}\right) .
\end{aligned}
$$

Par conséquent,

$$
\operatorname{deg}(M) \leqslant \frac{\operatorname{deg}_{X}\left(c_{1}(L) c_{1}(\mathscr{L})^{d}\right)}{\operatorname{deg}_{\mathscr{L}_{K^{\prime}}}\left(X_{K^{\prime}}\right)} n .
$$

Enfin, d'après la comparaison que l'on a établi dans la proposition 4.3.4, on déduit la majoration linéaire en $n$ de $\mu_{\max }\left(\pi_{*}\left(\mathscr{L}^{\otimes n}\right)\right)$ annoncée.

ThÉORÈme 4.3.6. - Soient $\pi: X \rightarrow C$ un morphisme projectif et plat, et $L$ un fibré inversible sur $X$. On suppose que $H^{0}\left(X_{K^{\prime}}, L_{K^{\prime}}^{\otimes n}\right)$ est non-nul lorsque $n$ est assez grand. Pour tout entier $n \geqslant 1$, soit $B_{n}=\pi_{*}\left(L^{\otimes n}\right)$.

1) La suite $\left(\mu_{\max }\left(B_{n}\right) / n\right)_{n \geqslant 1}$ converge vers un nombre dans $\mathbb{R}$. 
2) Si l'algèbre $\bigoplus_{n \geqslant 0} H^{0}\left(X_{K^{\prime}}, L_{K^{\prime}}^{\otimes n}\right)$ est de type fini sur $K^{\prime}$, alors la suite de polygones de Harder-Narasimhan $\left(\mathscr{P}_{B_{n}} / n\right)_{n \geqslant 1}$ converge uniformément vers une fonction concave $\mathscr{P}_{L}^{\pi}$, et on a

$$
\lim _{n \rightarrow+\infty} \frac{(d+1) !}{n^{d+1}} \chi\left(B_{n}\right)=(d+1) \operatorname{vol}\left(L_{K^{\prime}}\right) \mathscr{P}_{L}^{\pi}(1) .
$$

Si $L$ est ample, alors

$$
\mathscr{P}_{L}^{\pi}(1)=\frac{c_{1}(L)^{d+1}}{(d+1) c_{1}\left(L_{K^{\prime}}\right)^{d}} .
$$

3) Si l'homomorphisme canonique

$$
\left.H^{0}\left(X_{K^{\prime}}\right), L_{K^{\prime}}^{\otimes n}\right) \otimes H^{0}\left(X_{K^{\prime}}, L_{K^{\prime}}^{\otimes n}\right) \rightarrow H^{0}\left(X_{K^{\prime}}, L_{K^{\prime}}^{\otimes(m+n)}\right)
$$

est surjectif pous tous $n$ et $m$ assez grand, alors $\left(\mu_{\min }\left(B_{n}\right) / n\right)_{n \geqslant 1}$ converge dans $\mathbb{R}$.

Démonstration. - D'après la proposition 4.3.5, les conditions du théorème 4.3.2 sont vérifiées pour l'algèbre $B=\bigoplus_{n \geqslant 0} \pi_{*}\left(L^{\otimes n}\right)$. On obtient donc les résultats sauf les égalités (79) et (80). On a $\lim _{n \rightarrow+\infty} \mu\left(B_{n}\right) / n=\mathscr{P}_{L}^{\pi}(1)$. En outre, d'après (78),

$$
\chi\left(B_{n}\right)=\operatorname{deg}\left(B_{n}\right)+\operatorname{rg}\left(B_{n}\right)(1-g),
$$

d'où

$$
\lim _{n \rightarrow+\infty} \frac{\chi\left(B_{n}\right)}{n \operatorname{rg}\left(B_{n}\right)}=\lim _{n \rightarrow+\infty} \frac{\mu\left(B_{n}\right)}{n} .
$$

Comme $\lim _{n \rightarrow \infty} d ! \operatorname{rg}\left(B_{n}\right) / n^{d}=\operatorname{vol}\left(L_{K^{\prime}}\right)$, on obtient (79). Enfin, lorsque $L$ est ample, (80) se déduit du théorème de Riemann-Roch-Grothendieck.

4.3.3. Pente maximale asymptotique. - Soit $\pi: X \rightarrow C$ un $k$-morphisme projectif et plat d'une variété projective $X$ vers une courbe projective régulière $C$. On note $K^{\prime}=k(C)$ le corps des fonctions rationnelles sur $C$. Pour tout $\theta_{X^{-}}$ module inversible $L$ tel que $L_{K^{\prime}}$ soit gros, le théorème 4.3 .6 montre que la suite $\left(\mu_{\max }\left(\pi_{*} L^{\otimes n}\right) / n\right)_{n \geqslant 1}$ converge dans $\mathbb{R}$. On désigne par $\mu_{\max }^{\pi}(L)$ cette limite. Dans la suite, on montrera que la fonction $\mu_{\max }^{\pi}$ est sur-additive. Ce résultat permet d'étendre le domaine de définition de $\mu_{\max }^{\pi}$ à $\operatorname{Pic}(X)$ - le groupe des classes d'isomorphisme de fibrés inversibles sur $X$.

Lemme 4.3.7. - Soient $E_{1}$ et $E_{2}$ deux $\theta_{X}$-modules localement libres de rang fini et non-nuls. Si $\pi_{*}\left(E_{1}\right)$ et $\pi_{*}\left(E_{2}\right)$ sont tous les deux non-nuls, alors on a

$$
\mu_{\max }\left(\pi_{*}\left(E_{1} \otimes E_{2}\right)\right) \geqslant \mu_{\max }\left(\pi_{*} E_{1}\right)+\mu_{\max }\left(\pi_{*} E_{2}\right)-2 a(C),
$$

où $a(C)$ est la constante définie dans \$4.3.1. 
Démonstration. - Comme $\pi_{*}\left(E_{1}\right)$ et $\pi_{*}\left(E_{2}\right)$ sont non-nuls, d'après la proposition 4.3.4, il existe deux $\theta_{C}$-modules inversibles $M_{1}$ et $M_{2}$ ainsi que deux homomorphisme injectifs $M_{1} \longrightarrow \pi_{*}\left(E_{1}\right)$ et $M_{2} \longrightarrow \pi_{*}\left(E_{2}\right)$ tels que $\operatorname{deg} M_{1} \geqslant \mu_{\max }\left(\pi_{*} E_{1}\right)-a(C)$ et $\operatorname{deg} M_{2} \geqslant \mu_{\max }\left(\pi_{*} E_{2}\right)-a(C)$. Comme $M_{1}^{\vee} \otimes \pi_{*}\left(E_{1}\right)$ et $M_{2}^{\vee} \otimes \pi_{*}\left(E_{2}\right)$ ont des sections non partout nulles au-dessus de $C, \pi^{*}\left(M_{1}\right)^{\vee} \otimes E_{1}$ et $\pi^{*}\left(M_{2}\right)^{\vee} \otimes E_{2}$ ont des sections non partout nulles au-dessus de $X$. Par conséquent,

$$
H^{0}\left(X, \pi^{*}\left(M_{1} \otimes M_{2}\right)^{\vee} \otimes\left(E_{1} \otimes E_{2}\right)\right)=H^{0}\left(C,\left(M_{1} \otimes M_{2}\right)^{\vee} \otimes \pi_{*}\left(E_{1} \otimes E_{2}\right)\right) \neq 0 .
$$

Donc $0 \leqslant \mu_{\max }\left(\left(M_{1} \otimes M_{2}\right)^{\vee} \otimes \pi_{*}\left(E_{1} \otimes E_{2}\right)\right)$, et donc

$$
\mu_{\max }\left(\pi_{*}\left(E_{1} \otimes E_{2}\right)\right) \geqslant \operatorname{deg} M_{1}+\operatorname{deg} M_{2} \geqslant \mu_{\max }\left(\pi_{*}\left(E_{1}\right)\right)+\mu_{\max }\left(\pi_{*}\left(E_{2}\right)\right)-2 a(C) .
$$

Proposition 4.3.8. - Soient $L, L_{1}$ et $L_{2}$ des $\theta_{X}$-modules inversibles dont les fibres génériques sont gros.

1) Pour tout entier $n \geqslant 1, \mu_{\max }^{\pi}\left(L^{\otimes n}\right)=n \mu_{\max }^{\pi}(L)$.

2) Si $M$ est un $\theta_{C}$-module inversible, alors $\mu_{\max }^{\pi}\left(L \otimes \pi^{*} M\right)=\operatorname{deg} M+\mu_{\max }^{\pi}(L)$.

3) $\mu_{\max }^{\pi}\left(L_{1} \otimes L_{2}\right) \geqslant \mu_{\max }^{\pi}\left(L_{1}\right)+\mu_{\max }^{\pi}\left(L_{2}\right)$.

4) S'il existe un homomorphisme non-nul $\varphi: L_{1} \rightarrow L_{2}$ de $\theta_{X}$-modules, alors $\mu_{\max }^{\pi}\left(L_{1}\right) \leqslant \mu_{\max }^{\pi}\left(L_{2}\right)$.

Démonstration. - 1) est immédiat d'après la définition de $\mu_{\max }^{\pi}$.

2) On a, pour $n$ assez grand,

$$
\mu_{\max }\left(\pi_{*}\left(L^{\otimes n} \otimes \pi^{*} M^{\otimes n}\right)\right)=\mu_{\max }\left(\pi_{*}\left(L^{\otimes n}\right) \otimes M^{\otimes n}\right)=\mu_{\max }\left(\pi_{*}\left(L^{\otimes n}\right)\right)+n \operatorname{deg} M .
$$

D'où

$$
\lim _{n \rightarrow \infty} \frac{1}{n} \mu_{\max }\left(\pi_{*}\left(L^{\otimes n} \otimes \pi^{*} M^{\otimes n}\right)\right)=\operatorname{deg} M+\lim _{n \rightarrow \infty} \frac{1}{n} \mu_{\max }\left(\pi_{*}\left(L^{\otimes n}\right)\right) .
$$

3) D'après le lemme 4.3.7, pour tout entier $n$ assez grand,

$$
\mu_{\max }\left(\pi_{*}\left(L_{1}^{\otimes n} \otimes L_{2}^{\otimes n}\right)\right) \geqslant \mu_{\max }\left(\pi_{*}\left(L_{1}^{\otimes n}\right)\right)+\mu_{\max }\left(\pi_{*}\left(L_{2}^{\otimes n}\right)\right)-2 a(C) .
$$

Donc

$$
\frac{\mu_{\max }\left(\pi_{*}\left(L_{1}^{\otimes n} \otimes L_{2}^{\otimes n}\right)\right)}{n} \geqslant \frac{\mu_{\max }\left(\pi_{*}\left(L_{1}^{\otimes n}\right)\right)}{n}+\frac{\mu_{\max }\left(\pi_{*}\left(L_{2}^{\otimes n}\right)\right)}{n}-\frac{2 a(C)}{n} .
$$

Par passage à la limite on obtient $\mu_{\max }^{\pi}\left(L_{1} \otimes L_{2}\right) \geqslant \mu_{\max }^{\pi}\left(L_{1}\right)+\mu_{\max }^{\pi}\left(L_{2}\right)$.

4) Pour tout entier $n \geqslant 1$ on a un homomorphisme injectif $\varphi^{\otimes n}: L_{1}^{\otimes n} \rightarrow L_{2}^{\otimes n}$. En prenant l'image directe on obtient un homomorphisme injectif de $\pi_{*}\left(L_{1}^{\otimes n}\right)$ vers $\pi_{*}\left(L_{2}^{\otimes n}\right)$. Par conséquent, on a $\mu_{\max }\left(\pi_{*}\left(L_{1}^{\otimes n}\right)\right) \leqslant \mu_{\max }\left(\pi_{*}\left(L_{2}^{\otimes n}\right)\right)$. Par passage à la limite on obtient $\mu_{\max }^{\pi}\left(L_{1}\right) \leqslant \mu_{\max }^{\pi}\left(L_{2}\right)$.

DÉfinition 4.3.9. - Soient $L$ un $\vartheta_{X^{-}}$-module inversible et $\mathscr{L}$ un $\vartheta_{X}$-module inversible tel que $\mathscr{L}_{K^{\prime}}$ soit gros. D'après la proposition 1.4 .31 ), il existe un entier $n_{0}(L, \mathscr{L})>0$ tel que $L_{K^{\prime}} \otimes \mathscr{L}_{K^{\prime}}^{\otimes n}$ soit gros quel que soit $n \geqslant n_{0}(L, \mathscr{L})$. On définit, pour tout entier $n \geqslant n_{0}(L, \mathscr{L}), A_{n}(L, \mathscr{L})=\mu_{\max }^{\pi}\left(L \otimes \mathscr{L}^{\otimes n}\right)-n \mu_{\max }^{\pi}(\mathscr{L})$.

Proposition 4.3.10. - 1) La suite $\left(A_{n}(L, \mathscr{L})\right)_{n \geqslant n_{0}(L, \mathscr{L})}$ est croissante et converge dans $\mathbb{R}$. 
2) Si $M$ est un $\Theta_{C}$-module inversible, on a $A_{n}\left(L, \mathscr{L} \otimes \pi^{*} M\right)=A_{n}(L, \mathscr{L})$ pour tout $n \geqslant n_{0}(L, \mathscr{L})$.

3) On désigne par $\mathscr{C}$ l'ensemble des $\Theta_{X}$-modules inversibles génériquement gros et par $\mathscr{C}^{\prime}$ l'ensemble des $\Theta_{X}$-modules inversibles gros. Alors

$$
\inf _{\mathscr{L} \in \mathscr{C}} \lim _{n \rightarrow+\infty} A_{n}(L, \mathscr{L})=\inf _{\mathscr{L}^{\prime} \in \mathscr{Q}^{\prime}} \lim _{n \rightarrow+\infty} A_{n}\left(L, \mathscr{L}^{\prime}\right) .
$$

4) Si L est un $\vartheta_{X}$-module tel que $L_{K^{\prime}}$ soit gros, alors la valeur dans (81) est égale à $\mu_{\max }^{\pi}(L)$.

5) Si L est de la forme $\pi^{*} M$, où $M$ est un $\theta_{C}$-module inversible, alors la valeur dans (81) est égale à $\operatorname{deg}(M)$.

Démonstration. - 1) D'après la proposition 4.3.8 3), pour tout entier $n \geqslant n_{0}(L, \mathscr{L})$,

$$
\mu_{\max }^{\pi}\left(L \otimes \mathscr{L}^{\otimes(n+1)}\right) \geqslant \mu_{\max }^{\pi}\left(L \otimes \mathscr{L}^{\otimes n}\right)+\mu_{\max }^{\pi}(\mathscr{L}) .
$$

Donc on a $A_{n+1}(L, \mathscr{L}) \geqslant A_{n}(L, \mathscr{L})$. D'autre part, comme $\mathscr{L}_{K^{\prime}}$ est gros, il existe un $\vartheta_{C}$-module inversible $M$ tel que $\mathscr{L} \otimes \pi^{*} M$ soit gros (cf. la proposition 1.4.5 infra). Donc il existe un entier $m>0$ et un homomorphisme injectif de $L$ vers $\left(\mathscr{L} \otimes \pi^{*} M\right)^{\otimes m}$ (cf. proposition 1.4.3 infra). D'après la proposition 4.3.8 2) et 4), on a

$$
\mu_{\max }^{\pi}\left(L \otimes \mathscr{L}^{\otimes n}\right) \leqslant \mu_{\max }^{\pi}\left(\left(\mathscr{L} \otimes \pi^{*} M\right)^{\otimes m} \otimes \mathscr{L}^{\otimes n}\right)=(m+n) \mu_{\max }^{\pi}(\mathscr{L})+m \operatorname{deg}(M),
$$

i.e., $A_{n}(L, \mathscr{L}) \leqslant m\left(\mu_{\max }^{\pi}(\mathscr{L})+\operatorname{deg}(M)\right)$. La suite $\left(A_{n}(L, \mathscr{L})\right)_{n \geqslant n_{0}(L, \mathscr{L})}$ est alors croissante et bornée supérieurement, donc converge dans $\mathbb{R}$.

2) Si $L \otimes \mathscr{L}^{\otimes n}$ est génériquement gros, il en est de même de $L \otimes\left(\mathscr{L} \otimes \pi^{*} M\right)^{\otimes n}=$ $L \otimes \mathscr{L}^{\otimes n} \otimes \pi^{*} M^{\otimes n}$, et on a, d'après la proposition 4.3.8 2),

$$
\mu_{\max }^{\pi}\left(L \otimes\left(\mathscr{L} \otimes \pi^{*} M\right)^{\otimes n}\right)=\mu_{\max }^{\pi}\left(L \otimes \mathscr{L}^{\otimes n}\right)+n \operatorname{deg} M .
$$

Par conséquent,

$$
A_{n}\left(L, \mathscr{L} \otimes \pi^{*} M\right)=\mu_{\max }^{\pi}\left(L \otimes \mathscr{L}^{\otimes n}\right)+n \operatorname{deg}(M)-n \mu_{\max }^{\pi}\left(\mathscr{L} \otimes \pi^{*} M\right)=A_{n}(L, \mathscr{L}) .
$$

3) L'égalité (81) est une conséquence immédiate de 2) et de la proposition 1.4.5.

4) Pour tout $\vartheta_{X}$-module inversible génériquement gros $\mathscr{L}$, d'après la proposition 4.3.8 3), on a

$$
\mu_{\max }^{\pi}\left(L \otimes \mathscr{L}^{\otimes n}\right)-n \mu_{\max }^{\pi}(\mathscr{L}) \geqslant \mu_{\max }^{\pi}(L)+\mu_{\max }^{\pi}\left(\mathscr{L}^{\otimes n}\right)-n \mu_{\max }^{\pi}(\mathscr{L})=\mu_{\max }^{\pi}(L) .
$$

Donc on a $\inf _{\mathscr{L} \in \mathscr{Q}} \lim _{n \rightarrow+\infty} A_{n}(L, \mathscr{L}) \geqslant \mu_{\max }^{\pi}(L)$. D'autre part, comme $L_{K^{\prime}}$ est gros,

$$
\begin{aligned}
& \inf _{\mathscr{L} \in \mathscr{Q} n \rightarrow+\infty} \lim _{n} A_{n}(L, \mathscr{L}) \leqslant \lim _{n \rightarrow+\infty} A_{n}(L, L) \\
= & \lim _{n \rightarrow+\infty}\left(\mu_{\max }^{\pi}\left(L \otimes L^{\otimes n}\right)-n \mu_{\max }^{\pi}(L)\right)=\mu_{\max }^{\pi}(L) .
\end{aligned}
$$

On obtient donc l'égalité. 
5) Si $L=\pi^{*} M$, où $M$ est un $\vartheta_{C}$-module inversible, alors pour tout $\Theta_{X}$-module inversible génériquement gros $\mathscr{L}$ et tout entier $n>0$, on a, d'après la proposition 4.3.8 2)

$$
\begin{aligned}
& A_{n}(L, \mathscr{L})=\mu_{\max }^{\pi}\left(\pi^{*} M \otimes \mathscr{L}^{\otimes n}\right)-n \mu_{\max }^{\pi}(\mathscr{L}) \\
= & \operatorname{deg}(M)+\mu_{\max }^{\pi}\left(\mathscr{L}^{\otimes n}\right)-n \mu_{\max }^{\pi}(\mathscr{L})=\operatorname{deg}(M) .
\end{aligned}
$$

DÉfinition 4.3.11. - Pour tout $\theta_{X}$-module inversible $L$ on définit

$$
\mu_{\max }^{\pi}(L)=\inf _{\mathscr{L}} \lim _{n \rightarrow+\infty}\left(\mu_{\max }^{\pi}\left(L \otimes \mathscr{L}^{\otimes n}\right)-n \mu_{\max }^{\pi}(\mathscr{L})\right) \in[-\infty,+\infty[,
$$

où $\mathscr{L}$ parcourt tous les $\theta_{X}$-modules inversibles tels que $\mathscr{L}_{K^{\prime}}$ soit gros. Lorsque $L_{K^{\prime}}$ est gros, cette valeur est finie, et coïncide avec la limite $\lim _{n \rightarrow+\infty} \mu_{\max }\left(L^{\otimes n}\right) / n$ (cf. la proposition 4.3.10 4)). La valeur $\mu_{\max }^{\pi}(L)$ est appelée la pente maximale asymptotique de $L$ relativement à $\pi$.

La proposition suivante montre que les propriétés de la fonction $\mu_{\max }^{\pi}$ établie dans la proposition 4.3.8 sont encore valables pour la fonction généralisées.

Proposition 4.3.12. - 1) Pour tous $\theta_{X}$-modules inversibles $L_{1}$ et $L_{2}$, on a

$$
\mu_{\max }^{\pi}\left(L_{1} \otimes L_{2}\right) \geqslant \mu_{\max }^{\pi}\left(L_{1}\right)+\mu_{\max }^{\pi}\left(L_{2}\right) .
$$

2) Pour tout $\theta_{X}$-module inversible $L$ et tout entier $n>0$, on a

$$
\mu_{\max }^{\pi}\left(L^{\otimes n}\right)=n \mu_{\max }^{\pi}(L) .
$$

3) Si $L_{1}$ et $L_{2}$ sont deux $\vartheta_{X}$-modules inversibles et s'il existe un homomorphisme non-nul de $L_{1}$ vers $L_{2}$, alors $\mu_{\max }^{\pi}\left(L_{1}\right) \leqslant \mu_{\max }^{\pi}\left(L_{2}\right)$.

4) Pour tout $\theta_{X}$-module inversible $L$ et tout $\theta_{C}$-module inversible $M$, on a

$$
\mu_{\max }^{\pi}\left(L \otimes \pi^{*} M\right)=\mu_{\max }^{\pi}(L)+\operatorname{deg}(M) .
$$

Démonstration. - 1) Pour tout $\Theta_{X}$-module inversible $\mathscr{L}$ génériquement gros et tout entier $n$ suffisamment grand, d'après la proposition 4.3.8 3),

$$
\mu_{\max }^{\pi}\left(L_{1} \otimes L_{2} \otimes \mathscr{L}^{\otimes 2 n}\right) \geqslant \mu_{\max }^{\pi}\left(L_{1} \otimes \mathscr{L}^{\otimes n}\right)+\mu_{\max }^{\pi}\left(L_{2} \otimes \mathscr{L}^{\otimes n}\right),
$$

d'où $A_{2 n}\left(L_{1} \otimes L_{2}, \mathscr{L}\right) \geqslant A_{n}\left(L_{1}, \mathscr{L}\right)+A_{n}\left(L_{2}, \mathscr{L}\right)$. Par passage à la limite on obtient $\mu_{\max }^{\pi}\left(L_{1} \otimes L_{2}\right) \geqslant \mu_{\max }^{\pi}\left(L_{1}\right)+\mu_{\max }^{\pi}\left(L_{2}\right)$.

2) Pour tout $\vartheta_{X}$-module inversible $\mathscr{L}$ génériquement gros et tout entier $m$ assez grand, on a, d'après la proposition 4.3.8 1),

$$
\mu_{\max }^{\pi}\left(L^{\otimes n} \otimes \mathscr{L}^{\otimes m n}\right)=n \mu_{\max }^{\pi}\left(L \otimes \mathscr{L}^{\otimes m}\right) .
$$

Par conséquent, on a $A_{m n}\left(L^{\otimes n}, \mathscr{L}\right)=n A_{m}(L, \mathscr{L})$. Par passage à la limite, on obtient $\mu_{\max }^{\pi}\left(L^{\otimes n}\right)=n \mu_{\max }^{\pi}(L)$.

3) Pour tout $\vartheta_{X}$-module inversible $\mathscr{L}$ génériquement gros et tout entier $n$ suffisamment grand, on a un homomophisme injectif de $L_{1} \otimes \mathscr{L}^{\otimes n}$ vers $L_{2} \otimes \mathscr{L}^{\otimes n}$. Par la proposition 4.3.84), on a $\mu_{\text {max }}^{\pi}\left(L_{1} \otimes \mathscr{L}^{\otimes n}\right) \leqslant \mu_{\max }^{\pi}\left(L_{2} \otimes \mathscr{L}^{\otimes n}\right)$, qui implique que $A_{n}\left(L_{1}, \mathscr{L}\right) \leqslant A_{n}\left(L_{2}, \mathscr{L}\right)$. Par passage à la limite, on obtient $\mu_{\max }^{\pi}\left(L_{1}\right) \leqslant \mu_{\max }^{\pi}\left(L_{2}\right)$. 
4) Pour tout $\theta_{X}$-module inversible génériquement gros $\mathscr{L}$ et tout entier $n$ suffisamment grand, on a, d'après la 4.3.8 2),

$$
A_{n}\left(L \otimes \pi^{*} M, \mathscr{L}\right)=A_{n}(L, \mathscr{L})+\operatorname{deg}(M) .
$$

Par passage à la limite on obtient $\mu_{\max }^{\pi}\left(L \otimes \pi^{*} M\right)=\mu_{\max }^{\pi}(L)+\operatorname{deg}(M)$.

4.3.4. Pente maximale asymptotique et annulation de section globale. La négativité de la pente maximale asymptotique est liée à l'annulation de section globale de certains fibrés vectoriels. Les résultats présentés dans ce sous-paragraphe sont analogues à ceux dans $§ 4.2 .4$. On fixe dans ce sous-paragraphe un $\vartheta_{X}$-module inversible $L$.

Proposition 4.3.13. - 1) Si $\mu_{\max }^{\pi}(L)>0$, alors $\mu_{\max }^{\pi}\left(L^{\vee}\right)<0$.

2) Si $\mu_{\max }^{\pi}(L)<0$, alors $H^{0}(X, L)=0$.

3) Si L est gros, alors $\mu_{\max }^{\pi}(L)>0$.

Démonstration. - 1) On a $\mu_{\max }^{\pi}\left(\theta_{X}\right)=\mu_{\max }^{\pi}\left(\pi^{*} \theta_{C}\right)=\operatorname{deg}\left(\theta_{C}\right)=0$, donc $\mu_{\max }^{\pi}(L)+$ $\mu_{\max }^{\pi}\left(L^{\vee}\right) \leqslant 0$ compte tenu de la proposition 4.3.12 1).

2) Si $H^{0}(X, L) \neq 0$, alors il existe un homomorphisme non-nul de $\vartheta_{X}$ vers $L$, d'où $\mu_{\max }^{\pi}(L) \geqslant \mu_{\max }^{\pi}\left(\theta_{X}\right)=0$, compte tenu de la proposition 4.3.12 3).

3) Soit $M$ un $\theta_{C}$-module inversible tel que $\operatorname{deg}(M)>0$. Comme $L$ est gros, il existe un entier $n \geqslant 1$ tel que $\pi^{*} M^{\vee} \otimes L^{\otimes n}$ ait une section globale non-nulle. D'après 2 ), on a

$$
\mu_{\max }^{\pi}\left(\pi^{*} M^{\vee} \otimes L^{\otimes n}\right)=n \mu_{\max }^{\pi}(L)+\operatorname{deg}\left(M^{\vee}\right) \geqslant 0,
$$

Donc $\mu_{\max }^{\pi}(L) \geqslant \operatorname{deg}(M) / n>0$.

THÉORÈme 4.3.14. - Si $\mu_{\max }^{\pi}\left(L^{\vee}\right)<0$, alors la condition suivante est satisfaite :

il existe un $\vartheta_{X}$-module inversible gros $\mathscr{L}$ et un nombre réel $\lambda>0$ tel que, quels que soient les entiers $D>\lambda$ et $n>\lambda D, H^{0}\left(X, L^{\vee \otimes n} \otimes \mathscr{L}^{\otimes D}\right)=0$.

La réciproque est vraie lorsque $L_{K^{\prime}}^{\vee}$ est gros.

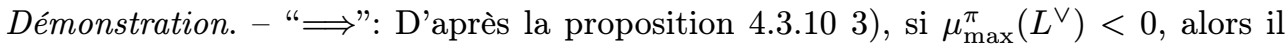
existe une constante $\varepsilon>0$ et un $\vartheta_{X}$-module gros $\mathscr{L}$ tels que, pour tout entier $m$ suffisamment grand, $A_{m}\left(L^{\vee}, \mathscr{L}\right) \leqslant-\varepsilon$. On a alors $\mu_{\max }^{\pi}\left(L^{\vee} \otimes \mathscr{L}\right) \leqslant \mu_{\max }^{\pi}(\mathscr{L})-\varepsilon$. Soit $\lambda$ une constante telle que $\lambda>\varepsilon^{-1} \mu_{\max }^{\pi}(\mathscr{L})$. Pour tout entier $D \geqslant 1$ et tout entier $n>\lambda D$, on obtient, d'après la proposition 4.3.12 1),

$$
\begin{aligned}
& (n-D) \mu_{\max }^{\pi}(\mathscr{L})+\mu_{\max }^{\pi}\left(L^{\vee \otimes n} \otimes \mathscr{L}^{\otimes D}\right) \leqslant \mu_{\max }^{\pi}\left(\left(L^{\vee} \otimes \mathscr{L}\right)^{\otimes n}\right) \\
= & n \mu_{\max }^{\pi}\left(L^{\vee} \otimes \mathscr{L}\right) \leqslant n\left(\mu_{\max }^{\pi}(\mathscr{L})-\varepsilon\right) .
\end{aligned}
$$

Par conséquent, on a

$$
\mu_{\max }^{\pi}\left(L^{\vee \otimes n} \otimes \mathscr{L}^{\otimes D}\right) \leqslant D \mu_{\max }^{\pi}(\mathscr{L})-n \varepsilon<0 .
$$

D'après la proposition 4.3.13 2), on a $H^{0}\left(X, L^{\vee \otimes n} \otimes \mathscr{L}^{\otimes D}\right)=0$. Ainsi $(X, L)$ satisfait à la condition. 
" $\Longleftarrow:$ On suppose que $L_{K^{\prime}}^{\vee}$ est gros et que la condition est vérifiée pour $L$. La condition dans le théorème est équivalente à la condition suivante :

pour tout $\Theta_{X}$-module inversible $\mathcal{L}$, il existe $\lambda>0$ tel que, quels que soient

les entiers $D>\lambda$ et $n>\lambda D$, on ait $H^{0}\left(X, L^{\vee \otimes n} \otimes \mathscr{L}^{\otimes D}\right)=0$.

En effet, si $\mathscr{L}$ est un $\bigoplus_{X}$-module inversible gros, alors il existe un homomorphisme injectif de $\mathscr{L}$ dans une puissance tensorielle $\mathscr{L}^{\otimes m}$. Donc l'annulation de $H^{0}\left(X, L^{\vee \otimes n} \otimes\right.$ $\left.\mathscr{L}^{\otimes m d}\right)$ implique celle de $H^{0}\left(X, L^{\vee \otimes n} \otimes \mathscr{L}^{\otimes D}\right)$.

Soit $M$ un $\theta_{C}$-module inversible ample. Il existe $\lambda>0$ tel que, pour tout entier $D>\lambda$ et tout entier $n>\lambda D$, on ait

$$
H^{0}\left(X, L^{\vee \otimes n} \otimes \pi^{*} M^{\otimes D}\right)=0 .
$$

D'après le lemme 4.3.3 et la proposition 4.3.12 4), on a

$$
\mu_{\max }\left(\pi_{*}\left(L^{\vee \otimes n} \otimes \pi^{*} M^{\otimes D}\right)\right)=D \operatorname{deg} M+\mu_{\max }\left(\pi_{*}\left(L^{\vee \otimes n}\right)\right) \leqslant g-1 .
$$

On prend une suite $\left(D_{n}\right)_{n \geqslant 1}$ telle que

i) pour tout entier $n \geqslant 1, D_{n}>\lambda$,

ii) pour $n$ suffisamment grand, $D_{n}<n / \lambda$,

iii) $\lim _{n \rightarrow \infty} \frac{n}{D_{n}}=\lambda$.

Alors pour $n$ suffisamment grand, on a $\operatorname{deg}(M) D_{n}+\mu_{\max }\left(\pi_{*}\left(L^{\vee \otimes n}\right)\right) \leqslant g-1$, c'està-dire

$$
\operatorname{deg}(M) \frac{D_{n}}{n}+\frac{\mu_{\max }\left(\pi_{*}\left(L^{\vee \otimes n}\right)\right)}{n} \leqslant \frac{g-1}{n} .
$$

Par passage à la limite, on obtient $\mu_{\max }^{\pi}\left(L^{\vee}\right) \leqslant-\lambda^{-1} \operatorname{deg} M<0$.

4.3.5. Pente maximale asymptotique d'un fibré vectoriel. - Par passage au fibré canonique sur le fibré projectif, on étend le domaine de définition de la fonction $\mu_{\text {max }}^{\pi}$ à l'ensemble des fibrés vectoriels sur $X$. Si $E$ est un $\theta_{X}$-module localement libre de rang fini et non-nul et si $p: \mathbb{P}(E) \rightarrow X$ est le morphisme canonique, on définit

$$
\mu_{\max }^{\pi}(E)=\mu_{\max }^{\pi p}\left(\theta_{E}(1)\right) .
$$

Remarque 4.3.15. - Si $E_{K^{\prime}}$ est gros, c'est-à-dire que si $\theta_{E}(1)_{K^{\prime}}$ est gros, alors $\mu_{\max }^{\pi}(E)=\lim _{n \rightarrow \infty} \mu_{\max }\left(\pi_{*}\left(S^{n} E\right)\right)$.

LEMME 4.3.16. - Les deux conditions suivantes sont équivalentes :

1) pour tout $\theta_{X}$-module inversible $M$ et tout $\Theta_{X}$-module cohérent sans torsion $\mathcal{F}$, il existe un nombre réel $\lambda>0$ tels que, quels que soient les entiers $D>\lambda$ et $n>\lambda D$, on ait $H^{0}\left(X, S^{n} E^{\vee} \otimes M^{\otimes D} \otimes \mathcal{F}\right)=0$.

2) pour tout $\Theta_{X}$-module inversible $M$, il existe un nombre réel $\lambda>0$ tels que, quels que soient les entiers $D>\lambda$ et $n>\lambda D$, on ait $H^{0}\left(X, S^{n} E^{\vee} \otimes M^{\otimes D}\right)=0$.

3) il existe un $\vartheta_{X}$-module inversible gros $L$ et un nombre réel $\lambda>0$ tels que, quels que soient les entiers $D>\lambda$ et $n>\lambda D$, on ait $H^{0}\left(X, S^{n} E^{\vee} \otimes L^{\otimes D}\right)=0$. 


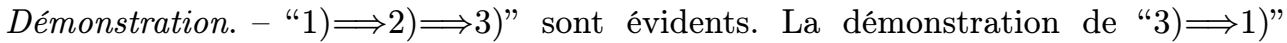
repose sur la proposition 1.4 .3 , et est très similaire à celle de son homologue dans la proposition 4.2.20.

THÉORÈme 4.3.17. - Si $\mu_{\max }^{\pi}\left(E^{\vee}\right)<0$, alors la condition suivante est vérifiée : pour tout $\theta_{X}$-module inversible $M$ (ou de façon équivalente, il existe un $\vartheta_{X}$-module inversible gros $\left.M\right)$, il existe un nombre réel $\lambda>0$ tels que, quels que soient les entiers $D>\lambda$ et $n>\lambda D$, on ait $H^{0}\left(X, S^{n} E^{\vee} \otimes\right.$ $\left.M^{\otimes D}\right)=0$.

La réciproque est vraie lorsque $E_{K^{\prime}}^{\vee}$ est gros, c'est-à-dire que le faisceau inversible $\vartheta_{E^{\vee}}(1)_{K^{\prime}}$ est gros.

Démonstration. - La condition dans ce théorème est équivalente à la condition dans le théorème 4.3.14 pour $\left(\mathbb{P}\left(E^{\vee}\right), \vartheta_{E^{\vee}}(-1)\right)$ :

pour tout $\Theta_{\mathbb{P}\left(E^{\vee}\right)}$-module inversible $L$ (ou de façon équivalente, il existe un $\vartheta_{\mathbb{P}\left(E^{\vee}\right)}$-module inversible gros $\left.L\right)$, il existe un nombre réel $\lambda>0$ tels que, quels que soient les entiers $D>\lambda$ et $n>\lambda D$, on ait $H^{0}\left(\mathbb{P}\left(E^{\vee}\right), \theta_{E^{\vee}}(n) \otimes\right.$ $\left.L^{\otimes D}\right)=0$.

En effet, si $M$ est un $\theta_{X}$-module inversible, alors

$$
H^{0}\left(\mathbb{P}\left(E^{\vee}\right), \theta_{E^{\vee}}(n) \otimes p^{*} M^{\otimes D}\right)=H^{0}\left(X, S^{n} E^{\vee} \otimes M^{\otimes D}\right) .
$$

Donc l'annulation de $H^{0}\left(\mathbb{P}\left(E^{\vee}\right), \vartheta_{E^{\vee}}(n) \otimes p^{*} M^{\otimes D}\right)$ implique celle de $H^{0}\left(X, S^{n} E^{\vee} \otimes\right.$ $\left.M^{\otimes D}\right)$. Réciproquement, d'après la proposition 1.4.5, il existe un $\theta_{X}$-module inversible $M$ tel que $L:=\theta_{E^{\vee}}(1) \otimes p^{*} M$ soit gros. On a $H^{0}\left(\mathbb{P}\left(E^{\vee}\right), \vartheta_{E^{\vee}}(n) \otimes\right.$ $\left.L^{\otimes D}\right)=H^{0}\left(\mathbb{P}\left(E^{\vee}\right), \theta_{E^{\vee}}(n+D) \otimes p^{*} M^{\otimes D}\right)=H^{0}\left(X, S^{n+D} E^{\vee} \otimes M^{\otimes D}\right)$. Si ce dernier espace s'annule lorsque $n / D$ est assez grand, alors il en est de même de $H^{0}\left(\mathbb{P}\left(E^{\vee}\right), \theta_{E^{\vee}}(n) \otimes L^{\otimes D}\right)$.

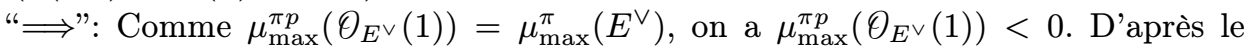
théorème 4.3.14, la condition comme ci-dessus est vérifiée.

"£": Si la condition dans ce théorème est vérifiée pour $(X, E)$, alors la condition dans le théorème 4.3.14 est vérifiée pour $\left(\mathbb{P}\left(E^{\vee}\right), \theta_{E^{\vee}}(-1)\right)$. Si de plus $E_{K^{\prime}}^{\vee}$ est gros, alors $\theta_{E^{\vee}}(-1)_{K^{\prime}}^{\vee}=\theta_{E^{\vee}}(1)_{K^{\prime}}$ est gros. Le théorème 4.3 .14 implique alors que $\mu_{\max }^{\pi p}\left(\theta_{E^{\vee}}(-1)^{\vee}\right)=\mu_{\max }^{\pi p}\left(\theta_{E^{\vee}}(1)\right)=\mu_{\max }^{\pi}\left(E^{\vee}\right)<0$. 


\section{BIBLIOGRAPHIE}

[1] A. Abbes \& T. Bouche - "Théorème de Hilbert-Samuel "arithmétique"", Ann. Inst. Fourier (Grenoble) 45 (1995), p. 375-401.

[2] P. Autissier - "Points entiers sur les surfaces arithmétiques", J. reine angew. Math. 531 (2001), p. 201-235.

[3] E. Bombieri \& J. VAaler - "On Siegel's lemma", Invent. Math. 73 (1983), p. 11-32.

[4] _ , "Addendum to: "On Siegel's lemma"”, Invent. Math. 75 (1984), p. 377.

[5] T. BoreK - "Successive minima and slopes of Hermitian vector bundles over number fields", J. Number Theory 113 (2005), p. 380-388.

[6] J.-B. Bost - "Périodes et isogenies des variétés abéliennes sur les corps de nombres (d'après D. Masser et G. Wüstholz)", Astérisque 237 (1996), p. 115161, Séminaire Bourbaki, Vol. 1994/95, exp. nº 795.

[7] _ , "Algebraic leaves of algebraic foliations over number fields", Publ. Math. Inst. Hautes Études Sci. 93 (2001), p. 161-221.

[8] _ - "Germs of analytic varieties in algebraic varieties: canonical metrics and arithmetic algebraization theorems", in Geometric aspects of Dwork theory. Vol. I, II, Walter de Gruyter GmbH \& Co. KG, Berlin, 2004, p. 371-418.

[9] _ "Evaluation maps, slopes, and algebraicity criteria", in International Congress of Mathematicians. Vol. II, Eur. Math. Soc., Zürich, 2006, p. 537-562.

[10] J.-B. Bost, H. Gillet \& C. Soulé - "Heights of projective varieties and positive Green forms", J. Amer. Math. Soc. 7 (1994), p. 903-1027.

[11] J.-B. Bost \& K. KÜnnemann - "Hermitian vector bundles and extension groups on arithmetic schemes. I. Geometry of numbers", Adv. Math. 223 (2010), p. $987-1106$.

[12] N. Bourbaki - Éléments de mathématique. Fasc. XIII. Livre VI: Intégration. Chapitres 1, 2, 3 et 4: Inégalités de convexité, Espaces de Riesz, Mesures sur les 
espaces localement compacts, Prolongement d'une mesure, Espaces $L^{p}$, Deuxième édition revue et augmentée. Actualités Scientifiques et Industrielles, No. 1175, Hermann, 1965.

[13] _ Éléments de mathématique. Algèbre. Chapitres 1 à 3, Hermann, 1970.

[14] _ Éléments de mathématique, Masson, 1998, Algèbre commutative. Chapitre 8. Dimension. Chapitre 9. Anneaux locaux noethériens complets.

[15] A. Chambert-Loir - "Théorèmes d'algébricité en géométrie diophantienne (d'après J.-B. Bost, Y. André, D. \& G. Chudnovsky)", Astérisque 282 (2002), p. 175-209, Séminaire Bourbaki, Vol. 2000/2001, exposé no 886.

[16] H. Chen - "Positivité en géométrie algébrique et en géométrie d'Arakelov : application à l'algébrisation et à l'étude asymptotique des polygones de HarderNarasimhan", Thèse, École polytechnique, 2006.

[17] _ "Maximal slope of tensor product of Hermitian vector bundles", $J$. Algebraic Geom. 18 (2009), p. 575-603.

[18] T. Chinburg - "Capacity theory on varieties", Compositio Math. 80 (1991), p. $75-84$.

[19] D. Eisenbud - Commutative algebra, Graduate Texts in Math., vol. 150, Springer, 1995.

[20] G. Faltings \& G. WÜstholz - "Diophantine approximations on projective spaces", Invent. Math. 116 (1994), p. 109-138.

[21] M. Fekete - "Über die Verteilung der Wurzeln bei gewissen algebraischen Gleichungen mit ganzzahligen Koeffizienten", Math. Z. 17 (1923), p. 228-249.

[22] É. Gaudron - "Pentes des fibrés vectoriels adéliques sur un corps global", Rend. Semin. Mat. Univ. Padova 119 (2008), p. 21-95.

[23] H. Gillet \& C. Soulé - "An arithmetic Riemann-Roch theorem", Invent. Math. 110 (1992), p. 473-543.

[24] D. R. Grayson - "Reduction theory using semistability", Comment. Math. Helv. 59 (1984), p. 600-634.

[25] A. Grothendieck - "Éléments de géométrie algébrique. II. Étude globale élémentaire de quelques classes de morphismes", Publ. Math. I.H.É.S., vol. 8, 1961.

[26] _ _Éléments de géométrie algébrique. III. Étude cohomologique des faisceaux cohérents. I", Publ. Math. I.H.É.S., vol. 11, 1961. 
[27] _ _Éléments de géométrie algébrique. IV. Étude locale des schémas et des morphismes de schémas. I", Publ. Math. I.H.É.S., vol. 20, 1964.

[28] G. Harder \& M. S. Narasimhan - "On the cohomology groups of moduli spaces of vector bundles on curves", Math. Ann. 212 (1974/75), p. 215-248.

[29] R. Hartshorne - "Ample vector bundles", Publ. Math. I.H.É.S. 29 (1966), p. $63-94$.

[30] _ Ample subvarieties of algebraic varieties, Notes written in collaboration with C. Musili. Lecture Notes in Math., vol. 156, Springer, 1970.

[31] L. HÖRMANDER - An introduction to complex analysis in several variables, third éd., North-Holland Mathematical Library, vol. 7, North-Holland Publishing Co., 1990 .

[32] _ Notions of convexity, Progress in Math., vol. 127, Birkhäuser, 1994.

[33] R. LAzARSFeld - Positivity in algebraic geometry. I, Ergebnisse Math. Grenzg.. 3. Folge. A Series of Modern Surveys in Mathematics, vol. 48, Springer, 2004, Classical setting: line bundles and linear series.

[34] _ Positivity in algebraic geometry. II, Ergebnisse Math. Grenzg.. 3. Folge. A Series of Modern Surveys in Mathematics, vol. 49, Springer, 2004, Positivity for vector bundles, and multiplier ideals.

[35] H. Matsumura - Commutative ring theory, second éd., Cambridge Studies in Advanced Math., vol. 8, Cambridge Univ. Press, 1989.

[36] R. B. McFeAt - "Geometry of numbers in adele spaces", Dissertationes Math. Rozprawy Mat., vol. 88, 1971.

[37] A. MORIWAKI - "Arithmetic height functions over finitely generated fields", Invent. Math. 140 (2000), p. 101-142.

[38] H. Randriambololona - "Métriques de sous-quotient et théorème de HilbertSamuel arithmétique pour les faisceaux cohérents", J. reine angew. Math. 590 (2006), p. 67-88.

[39] R. Rumely - "On the relation between Cantor's capacity and the sectional capacity", Duke Math. J. 70 (1993), p. 517-574.

[40] R. Rumely \& C. F. Lau - "Arithmetic capacities on $\mathbf{P}^{N}$ ", Math. Z. 215 (1994), p. $533-560$.

[41] R. Rumely, C. F. Lau \& R. VARley - "Existence of the sectional capacity", Mem. Amer. Math. Soc., vol. 145, 2000. 
[42] U. StuhleR - "Eine Bemerkung zur Reduktionstheorie quadratischer Formen", Arch. Math. (Basel) 27 (1976), p. 604-610.

[43] A. C. Thompson - Minkowski geometry, Encyclopedia of Mathematics and its Applications, vol. 63, Cambridge Univ. Press, 1996.

[44] J. L. Thunder - "An adelic Minkowski-Hlawka theorem and an application to Siegel's lemma", J. reine angew. Math. 475 (1996), p. 167-185.

[45] S. Zhang - "Positive line bundles on arithmetic varieties", J. Amer. Math. Soc. 8 (1995), p. 187-221. 